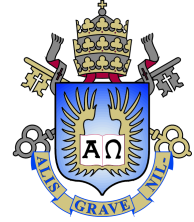

Raimundo Neto Nunes Leão

\title{
A geometria de espaços de polígonos generalizados
}

Tese apresentada como requisito parcial para obtenção do grau de Doutor pelo Programa de Pós-graduação em Matemáticada PUC-Rio.

Orientador : Prof. Marcos Craizer

Co-orientador: Profa. Alessia Mandini 


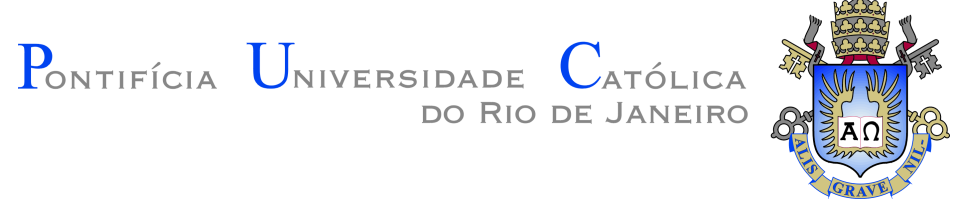

Raimundo Neto Nunes Leão

\title{
A geometria de espaços de polígonos generalizados
}

Tese apresentada como requisito parcial para obtenção do grau de Doutor pelo Programa de Pós-graduação em Matemáticada PUC-Rio. Aprovada pela Comissão Examinadora abaixo assinada.

\author{
Prof. Marcos Craizer \\ Orientador \\ Departamento de Matemática - PUC-Rio
}

Profa. Alessia Mandini

Co-orientador Instituto de Matemática e Estatística - UFF

Prof. Alejandro Cabrera Instituto de Matemática - UFRJ

Prof. David Francisco Martínez Torres

Departamento de Matemática - PUC-Rio

Prof. Gonçalo Marques Fernandes de Oliveira Instituto de Matemática e Estatística - UFF

Prof. Simon George Chiossi Instituto de Matemática e Estatística - UFF

Prof. Thiago Linhares Drummond Instituto de Matemática - UFRJ

Rio de Janeiro, 1 de Outubro de 2020 
Todos os direitos reservados. É proibida a reprodução total ou parcial do trabalho sem autorização da universidade, do autor e do orientador.

\section{Raimundo Neto Nunes Leão}

Licenciatura plena em Matemática pela Universidade Federal do Pará (UFPA) em 2010. Mestrado em Matemática pela Universidade Federal do Pará (UFPA) em 2014, com ênfase em Geometria.

Ficha Catalográfica

Leão, Raimundo Neto Nunes

A geometria de espaços de polígonos generalizados / Raimundo Neto Nunes Leão; orientador: Marcos Craizer; co-orientador: Alessia Mandini. - Rio de janeiro: PUC-Rio, Departamento de Matemática, 2020.

v., $147 \mathrm{f}$ : il. color. ; $30 \mathrm{~cm}$

Tese (doutorado) - Pontifícia Universidade Católica do Rio de Janeiro, Departamento de Matemática.

Inclui bibliografia

1. Matemática - Teses. 2. Espaço de moduli de polígonos;. 3. Variedade de Poisson;. 4. Órbita coadjunta.. I. Craizer, Marcos. II. Pontifícia Universidade Católica do Rio de Janeiro. Departamento de Matemática. III. Título. 
Para as Hannas da minha vida.

A Hellen Hanna por ser uma esposa maravilhosa.

E a nossa filha, Hanna Luise, que fez parte de uma forma muito especial. 


\section{Agradecimentos}

A Deus, pela oportunidade sem igual, por todos os benefícios, sustento, saúde, surpresas maravilhosas ao longo da vida e pela companhia formidável em momentos turbulentos.

Aos meus pais Manoel M. Leão e Priscila N. Leão pela criação, esforço e carinho, que mesmo em condições financeira mínima sempre foram muito honrados.

A minha esposa, companheira e amiga Hellen Hanna que decidiu seguir esse árduo caminho, simplesmete acreditando, incentivando e dando todo apoio, carinho e consolo.

A todos os professores que contribuíram na minha formação, em destaque meu pai, Manoel Leão, professor no primário no Caliçado em Cametá-PA; a professora Adma da escola Mário Barbosa, sendo esta essencial para minha escolha por licenciatura em Matemática; a professora Nazaré Bezerra, minha orientadora no TCC; ao professor Marcos M. Diniz da UFPA por ter sido brilhante orientador no mestrado; a professora Cristina Vaz por ser uma professora diferenciada e pela carta de recomendção pra estudar na PUC-Rio; ao professor Marcos Craizer por ter sido o primeiro orientador acadêmico na PUC; a professora Alessia Mandini, a principal responsável por esta tese, através dela tive a oportunidade de conhecer diversos pesquisadores renomados na área e pude aprender uma parte sensacional da matemática. Muito obrigado pela paciência e dedicação.

Aos membros da banca examinadora pelas valiosas contribuições afim de tornar esta tese um trabalho melhor.

A todos os amigos e companheiros de caminhada, em especial ao Fernando Bruno M. Nunes, amigo de graduação; Lidiane Carvalho, Liliane Coelho, Márcio Almeida, amigos de tutoria da UFPA; Sebástian Alejandro, Viviana Grajales, Marcelo Santos e Fabíola Valéria, amigos que conseguir na PUC-Rio.

Aos professores e toda equipe do departamento de Matemática da PUC-Rio, em particular a Creuza Nascimento pela maravilhosa pessoa que é e pelo excelente trabalho que faz. A todos os responsáveis por tornar o ambiente de estudo da PUC-Rio, especificamente o departamento de Matemática, um local excelente para estudo e pesquisa.

A PUC-Rio pela bolsa de isenção recebida.

O presente trabalho foi realizado com apoio da Coordenação de Aperfeiçoamento de Pessoal de Nível Superior - Brasil (CAPES) - Código de Financiamento 001. 
Aos amigos e irmãos em Cristo da congregação da Timbó, em Belém do Pará, e da IBNH, no Rio de Janeiro, em especial o pastor Janson Serafim.

A todos os meus sete irmãos, Manoel Junihor, exemplo de pessoa e que foi um excelente tutor por alguns meses; Jardson de Jesus, de quem aprendir a ser calmo e paciente; Julita Maria, por ter sido uma mãe e conselheira na adolescência; Benedito Neto, por ser corajoso, destemido e trabalhador; Jefferson Nunes, Juliane Nunes e Emely Nunes, pela responsabilidade de tentar ser exemplo pra eles. Estes sete fizeram parte desta conquista e juntamente com nossos pais formamos a família Leão.

A minha filha Hanna Luise, por todo sorriso encantador e inocente. Te amo filha! 


\section{Resumo}

Leão, Raimundo Neto Nunes; Craizer, Marcos; . A geometria de espaços de polígonos generalizados. Rio de Janeiro, 2020. 147p. Tese de Doutorado - Departamento de Matemática, Pontifícia Universidade Católica do Rio de Janeiro.

Espaços de moduli de polígonos em $\mathbb{R}^{3}$ com comprimento dos lados fixados é um exemplo amplamente estudado de variedade simplética. Esses espaços podem ser descritos como quociente simplético de um número finito de órbitas coadjuntas pelo grupo $S U(2)$. Nesta tese esses espaços de moduli são identificados como folhas simpléticas de uma variedade de Poisson que pode ser construída como quociente. Essa construção é a seguir generalizada ao caso de um produto de um número finito de órbitas coadjuntas pelo grupo $S U(n)$, e o resultado principal desse trabalho de tese descreve como esses espaços de moduli de polígonos generalizados formam uma folheação em folhas simpléticas de uma variedade de Poisson.

\section{Palavras-chave}

Espaço de moduli de polígonos; Variedade de Poisson; Órbita coadjunta. 


\section{Abstract}

Leão, Raimundo Neto Nunes; Craizer, Marcos (Advisor); (Co-Advisor). The geometry of generalized polygon spaces. Rio de Janeiro, 2020. 147p. Tese de doutorado - Departamento de Matemática, Pontifícia Universidade Católica do Rio de Janeiro.

Moduli spaces of polygons in $\mathbb{R}^{3}$ with fixed sides length are a widely studied example of symplectic manifold that can be described as the symplectic quotient of a finite number of $S U(2)$-coadjoint orbits by the diagonal action of the group $S U(2)$. In this thesis these spaces are identified as the symplectic leaves of a Poisson manifold, that can itself be obtained by a quotient procedure. The construction is then generalized to the case of the quotient of a product of finitely many $S U(n)$-coadjoint orbits by the diagonal action of $S U(n)$, and the main result of this thesis describes how these moduli spaces of generalized polygons fit together as the symplectic leaves of a quotient Poisson manifold.

\section{Keywords}

Moduli space of polygons; Poisson manifold; Coadjoint orbit. 


\section{Sumário}

1 Introdução 11

2 Variedades Simpléticas $\quad 16$

$3 \quad$ Espaço de moduli de polígonos em $\mathbb{R}^{3}$. 29

4 Variedades de Poisson $\quad 31$

4.1 O colchete de Schouten 43

4.2 Quocientes 48

$5 \quad$ A ação coadjunta de $S U(2)$ em $\mathfrak{s u}^{*}(2) \quad 51$

$6 \quad S U(n)$ e suas órbitas coadjunta $\quad 63$

6.1 Grupos de Lie de matrizes e as álgebras de Lie $\mathfrak{o}(n), \mathfrak{u}(n)$ e $\mathfrak{s u}(n) \quad 63$

6.2 A órbita coadjunta de SU(n) é difeomórfica a uma variedade bandeira 72

6.2.1 Variedade Bandeira $\quad 75$

6.3 Base da álgebra de Lie de $\mathfrak{s u}(n) \quad 80$

6.4 $S U(n)$ agindo em $\prod_{j=1}^{m} \mathfrak{s u}^{*}(n)$

7 Estratificação de Poisson $\quad 88$

$\begin{array}{lll}7.1 & \text { Exemplos } & 101\end{array}$

7.1.1 Estratificação das variedades $\mathfrak{s u}^{*}(2), \prod_{j=1}^{m} \mathfrak{s u}(2)$ e $\prod_{j=1}^{m} \mathbb{S}_{\left|\lambda_{j}\right|}^{2} \quad 101$

7.1.2 Estratificação das variedades $\mathfrak{s u}^{*}(n), \prod_{j=1}^{m} \mathfrak{s u} *^{*}(n) \quad 110$

$\begin{array}{lll}7.2 & \text { Folhas simpléticas das estratas } & 118\end{array}$

7.3 Folheação simplética de $\left(\prod_{j=1}^{m} \mathbb{F}_{\mathbb{C}}^{j}\left(d_{1}^{j}, \ldots, d_{r_{j}}^{j}\right)\right) / S U(n) \quad 121$

7.3.1 Espaço de moduli de polígonos para $S U(2)$ e $S U(n) \quad 130$

$7.4 \quad$ Subvariedades especiais de $M=\prod_{j=1}^{m} \mathfrak{s u}^{*}(n) \quad 131$

$\begin{array}{lll}\text { 7.5 Retornando aos exemplos } & 134\end{array}$

7.5.1 Folha simplética da estrata $c\left(\mathfrak{s u}^{*}(2)_{(\mathbb{T})} / S U(2)\right) \quad 135$

7.5.2 Folha simplética da estrata $c\left(\mathfrak{s u}^{*}(n)_{\left(G_{d^{j}}\right)} / S U(n)\right) \quad 135$

8 Anexo $\quad 137$

8.1 Álgebras de Lie e a forma de Killing 138

$\begin{array}{ll}\text { A Notações } & 147\end{array}$ 


\section{Lista de figuras}

Figura 2.1 Álgebra de Lie identificado com o espaço tangente 


\section{1 \\ Introdução}

Com raízes na física matemática, as geometrias simpléticas e de Poisson viveram uma vivaz expansão nos últimos 50 anos. Um argumento importante nessas áreas é a construção de quocientes simpléticos e de Poisson, isto é, dada uma variedade simplética ou de Poisson equipada com a ação de um grupo, usar a estrutura em órbitas para construir uma nova variedade que herde a estrutura simplética e de Poisson.

Os principais resultados desta tese são obtidos a partir da ação do grupo especial unitário $S U(n)$ no produto de $m$ variedades bandeira

$$
\mathcal{S}=\prod_{j=1}^{m} \mathbb{F}_{\mathbb{C}}^{j}\left(d_{1}^{j}, \ldots, d_{r_{j}}^{j}\right) .
$$

Analisamos três casos, no primeiro onde $n=2$, obtemos que os espaços reduzidos $\mu^{-1}(\xi) / S U(2)_{\xi}$, onde $\mu$ é o mapa momento, são subespaços estratificados simpléticos cujas estratas são folhas simpléticas de $\mathcal{S} / S U(2)$. Para os outros casos, com $2<n<\infty$, dependendo da variedade $\mathcal{S}$ inicial, teremos a ação coadjunta diagonal de $S U(n)$ livre ou não. Para o caso em que a ação é livre, o espaço de polígonos de $m$ lados é realizado pelo quociente $\mu^{-1}(0) / S U(n)$ e devido $\mathcal{S}$ ser simplético, temos que as componentes conexas do quociente $\mu^{-1}(0) / S U(n)$, que denotaremos por $c\left(\mu^{-1}(0) / S U(n)\right)$, são folhas simpléticas de $\mathcal{S} / S U(n)$. Para o caso em que a ação não é livre, chegamos a conclusão de que $\mu^{-1}(\xi) / S U(n)_{\xi}$ são subespaços estratificados simpléticos cujas estratas são folhas simpléticas das estratas de $\mathcal{S} / S U(n)$. Vale ressaltar que em todos os casos, $\mu^{-1}(0) / S U(n)$ é suave se o vetor de comprimento for genérico, o que está diretamente ligado a escolha inicial da variedade $\mathcal{S}$.

Importante mencionar que dada a variedade $\mathcal{S}$, como acima, o quociente $\mathcal{S} / S U(n)$ admite uma estrutura de Poisson, independentemente da escolha de $n, 2 \leq n \leq \infty$, mais especificamente $\mathcal{S} / S U(n)$ possui uma estrutura de variedade estratificada onde a estratificação é Poisson, essas informações estão descritas no seguinte resultado

Teorema 1.1 Seja $\psi: G \times M \rightarrow M$ uma ação de Poisson própria. As componentes conexas do espaço reduzido $M_{(H)} / G$ formam uma estratificação de Poisson de $\left(M / G,\{\cdot, \cdot\}_{M / G}\right)$. 
este resultado foi extraído de [10] e é devido a Fernandes, Ortega e Ratiu e é conhecido como o Teorema de estratificação de Poisson, enunciamos e demonstramos no Teorema 7.22.

Esta tese está organizada da seguinte forma:

Nos Capítulos 2, 3 e 4 apresentamos as ferramentas matemáticas necessárias de Geometria de Poisson e Simplética e o espaço de moduli de polígonos afim de que se tenha um bom entendimento desta tese. Alguns resultados são demonstrados e outros apenas enunciados. Damos uma ênfase especial para a ação e órbita coadjunta além da estrutura de Poisson proveniente da estrutura simplética.

No capítulo 4, apresentamos na definição 4.10 a estrutura de Poisson na variedade que é produto de duas variedades de Poisson, e em (4.4) apresentamos a estrutura de Poisson na variedade $M=\mathfrak{g}^{*} \times \cdots \times \mathfrak{g}^{*}$, onde $M$ é o produto de $n$ duais de álgebra de Lie de dimensão finita $\mathfrak{g}^{*}$, esta estrutura produto será amplamente utilizada. É bem conhecido que a ação coadjunta é uma ação de Poisson, nesta tese fazemos uso da ação coadjunta diagonal, provamos na proposição 4.19, enunciada abaixo, que essa ação também é uma ação de Poisson.

Proposição 1.2 A ação coadjunta diagonal

$$
\begin{aligned}
\psi: G \times\left(\mathfrak{g}^{*}\right)^{n} & \longrightarrow\left(\mathfrak{g}^{*}\right)^{n} \\
(g, \xi) & \longmapsto \psi(g, \xi)=\left(\operatorname{Ad}_{g}^{*}\left(\xi_{1}\right), \ldots, \operatorname{Ad}_{g}^{*}\left(\xi_{n}\right)\right)
\end{aligned}
$$

é uma ação de Poisson.

No capítulo 5 damos ênfase para o estudo da ação coadjunta diagonal do grupo $S U(2)$, iniciamos exibindo uma órbita coadjunta de $S U(2)$ juntamente com sua 2-formas simplética, em seguida estudamos a ação coadjunta diagonal de $S U(2)$ em $\prod_{j=1}^{n} \mathfrak{s u}^{*}(2)$, a partir desta ação obtemos a subvariedade $\mathcal{S}_{r}$ que é a órbita coadjunta diagonal de $S U(2)$ por $X \in \prod_{j=1}^{n} \mathfrak{s u}^{*}(2)$, tomando a ação coadjunta diagonal de $S U(2)$ restrita a subvariedade $\mathcal{S}_{r}$, conseguimos obter o espaço $M_{r}$ dos $n$-gons de comprimento de lados fixados.

No Capítulo 6 estudamos alguns grupos de Lie de matrizes e suas respectivas álgebras de Lie, dando uma atenção especial para o grupo $S U(n)$, utilizamos um resultado conhecido que diz que a órbita coadjunta de $S U(n)$ por $\xi \in \mathfrak{s u}^{*}(n)$, denotada por $\mathcal{O}_{\xi}$, é difeomórfica a uma variedade bandeira complexa, $\mathbb{F}_{\mathbb{C}}\left(d_{1}, \ldots, d_{r}\right)$, caracterizada pela sequência $d=\left(d_{1}, \ldots, d_{r}\right)$ das multiplicidades dos autovalores do elemento $\xi$, a partir desse resultado exibimos explicitamente as órbitas para o caso $S U(2)$ e $S U(3)$. 
Ainda no capítulo 6 apresentamos uma base da álgebra de Lie de $\mathfrak{s u}(n)$ e explicitamos os estabilizadores de cada elemento dessa base, com esta informação chegamos a conclusão de que a ação coadjunta de $S U(n)$ não é livre. Em seguida damos início ao estudo da ação coadjunta de $S U(n)$ em $\prod_{j=1}^{m} \mathfrak{s u}^{*}(n)$, posteriormente tomamos a ação restrita a uma órbita dessa ação, nos possibilitando a obtenção do mapa momento associado $\mu$ por conseguinte a realização do espaço de polígonos obtido através do quociente $\mu^{-1}(0) / S U(n)$.

No Capítulo 7 apresentamos os resultados a respeito da estratificação simplética e de Poisson as quais são necessárias quando se estuda os quocientes $M / G$ e $M / / G$ para ações que são próprias mas não são livres, uma vez que se a ação for própria e livre, esses quocientes são variedades, no entanto se a ação não é livre, então esses espaços podem nem mesmo ser variedades, mas ainda são espaços de Hausdorff e possuem uma estrutura de variedade estratificada.

Um resultado muito importante no estudo de estratificação, Teorema 7.12, fornece as estratificações de $M$ e $M / G$ para o caso em que a ação é própria, a estratificação de $M / G$ é dada pela partição $\left\{c_{i}\left(M_{\left(H_{k}\right)} / G\right)\right\}_{i \in I^{\prime}}, k \in$ $\mathbb{N}$, onde $c_{i}$ indica a $i$-ésima componente conexa da subvariedade com tipo de órbita

$$
M_{\left(H_{j}\right)}=\left\{m \in M \mid G_{m} \in\left(H_{j}\right)\right\}
$$

sendo $\left(H_{j}\right)$ a classe de conjugação do subgrupo de isotropia $H_{j}$. Em seguida, enunciamos um resultado extraído de [10], devido a Fernandes, Ortega e Ratiu, onde eles mostram que as componentes conexas do espaço reduzido $M_{(H)} / G$ formam uma estratificação de Poisson de $\left(M / G,\{\cdot, \cdot\}_{M / G}\right)$ desde que a ação de $G$ em $M$ seja Poisson própria.

Uma vez apresentado a teoria de estratificação simplética e de Poisson, trabalhamos com alguns exemplos, estudamos inicialmente o grupo $S U(2)$, onde mostramos que a ação coadjunta não é livre mas é própria e portanto $\mathfrak{s u}^{*}(2) / S U(2)$ tem uma estrutura de variedade estratificada, exibimos com detalhes as estratas. Em seguida estudamos a ação coadjunta diagonal de $S U(2)$ em $\prod_{j=1}^{n} \mathfrak{s u}^{*}(2)$, assim como para $\mathfrak{s u} \mathfrak{u}^{*}(2)$, neste exemplo também a ação não é livre e portanto estudamos a estratificação do espaço $\left(\prod_{j=1}^{n} \mathfrak{s u}(2)\right) / S U(2)$.

Ainda no capítulo 7 , fazemos uso da variedade $\mathcal{S}=\prod_{j=1}^{m} \mathbb{F}_{\mathbb{C}}^{j}\left(d_{1}^{j}, \ldots, d_{r_{j}}^{j}\right)$, onde cada variedade bandeira é vista como uma órbita coadjunta de $S U(n)$, com o intuito de estudar a folheação simplética do espaço $\mathcal{S} / S U(n)$, a partir da escolha inicial das $m$ sequências $\left(d^{j}\right)$ obtivemos alguns resultados interessantes, 
como é o caso do Teorema 7.42 e principalmente o Teorema 7.44, os quais estão enunciados a seguir.

Para o primeiro resultado, ao menos uma das sequências não pode ser obtida por uma permutação de alguma das demais sequências, o que nos garante que a ação neste caso é livre.

Teorema 1.3 Considere a ação coadjunta diagonal de $S U(n)$ em $\mathcal{S}$, onde

$$
\mathcal{S}=\prod_{j=1}^{m} \mathbb{F}_{\mathbb{C}}^{j}\left(d_{1}^{j}, \ldots, d_{r_{j}}^{j}\right)
$$

tal que existem ao menos duas sequências $\left(d^{j}\right)$ e $\left(d^{k}\right)$ tal que uma não é permutação da outra. Essa ação é Poisson e admite mapa momento associado $\mu$ dado por

$$
\begin{aligned}
\mu: \mathcal{S} & \longrightarrow \mathfrak{s u}^{*}(n) \simeq \mathbb{R}^{n^{2}-1} \\
\xi & \longmapsto \mu(\xi)=\sum_{j=1}^{m} \xi_{j} .
\end{aligned}
$$

$\left(\mathcal{S}, \pi_{\mathcal{S}}, S U(n), \mu\right)$ é um $S U(n)$-espaço Hamiltoniano livre e próprio. Então $0 \in \mathfrak{s u}^{*}(n)$ é um valor regular de $\mu$ e $\mathcal{S} / / S U(n):=\mu^{-1}(0) / S U(n)$ é uma subvariedade de Poisson de $\left(\mathcal{S} / S U(n), \pi_{\mathcal{S} / S U(n)}\right)$, e uma vez que $\pi_{\mathcal{S}}$ é simplético, $\pi_{\mathcal{S} / / S U(n)}$ também é simplético e as componentes conexas de $\mathcal{S} / / S U(n)$ são folhas simpléticas de $\left(\mathcal{S} / S U(n), \pi_{\mathcal{S} / S U(n)}\right)$.

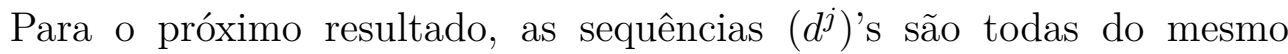
comprimento e diferem por uma permutação adequada, neste caso a ação não é livre. No teorema a seguir a variedade $\mathcal{S}$ é dada por

$$
\mathcal{S}=\prod_{j=1}^{m} \mathbb{F}_{\mathbb{C}}^{j}\left(d_{1}^{j}, \ldots, d_{r}^{j}\right)
$$

onde $d_{1}^{j}+\ldots+d_{r_{j}}^{j}=n$ para todo $j, \mathcal{S}$ admite uma estrutura de Poisson produto $\pi_{\mathcal{S}}$ a qual é não degenerada.

Teorema 1.4 Considere a ação coadjunta diagonal de $S U(n)$ em $\mathcal{S}$ a qual é Poisson e admite um mapa momento associado $\mu$ dado por

$$
\begin{aligned}
\mu: \mathcal{S} & \longrightarrow \mathfrak{s u}^{*}(n) \simeq \mathbb{R}^{n^{2}-1} \\
\xi & \longmapsto \mu(\xi)=\sum_{j=1}^{m} \xi_{j} .
\end{aligned}
$$

Nessas condições $\left(\mathcal{S}, \pi_{\mathcal{S}}, S U(n), \mu\right)$ é um $S U(n)$-espaço Hamiltoniano próprio, pois $\mu$ é $S U(n)$-equivariante. Além disso $\mathcal{S} / S U(n)$ é um espaço estratificado de Poisson em que as estratas são dadas pelas componentes conexas de 
$\mathcal{S}_{(H)} / S U(n)$, onde $H$ são subgrupos de isotropia da ação, e os espaços reduzidos $\mu^{-1}(\xi) / S U(n)_{\xi}$ são subespaços estratificados simpléticos cujas estratas são as folhas simpléticas das estratas de $\mathcal{S} / S U(n)$.

Finalmente no Capítulo 8 apresentamos alguns resultados que são úteis para consulta ao decorrer da leitura da tese, como é o caso de grupos de Lie semisimples, forma de Killing, propriedades da adjunta e equivalência entre as ações adjunta e coadjunta. 


\section{2}

\section{Variedades Simpléticas}

Neste capítulo enunciaremos alguns resultados de geometria simplética os quais serão relevantes para o desenvolvimento desta tese, daremos uma atenção especial às órbitas coadjuntas as quais são naturalmente variedades simpléticas.

Seja $M$ uma variedade suave, uma estrutura simplética em $M$ é uma 2 -forma fechada $\omega \in \Omega^{2}(M)$ que satisfaz $\omega^{n} \neq 0$, isto é, $\omega$ é uma forma não degenerada. Vale ressaltar que a não degenerescência de $\omega$ implica que $\operatorname{dim}(M)=2 n$ e $M$ deve ser uma variedade orientável.

Definição 2.1 Uma variedade simplética é um par $(M, \omega)$ composto por uma variedade suave $M$ dotada com uma estrutura simplética $\omega \in \Omega^{2}(M)$.

Vejamos alguns exemplos

Exemplo 2.2 Seja $M=\mathbb{R}^{2 n}$ com coordenadas lineares $x_{1}, \ldots, x_{n}, y_{1}, \ldots, y_{n}$. A forma

$$
\omega_{0}=\sum_{j=1}^{n} d x_{j} \wedge d y_{j}
$$

é uma forma simplética em $\mathbb{R}^{2 n}$.

Exemplo 2.3 Seja $M=\mathbb{C}^{n}$ com coordenadas lineares $z_{1}, \ldots, z_{n}$. A forma

$$
\omega=\frac{i}{2} \sum_{j=1}^{n} d z_{j} \wedge d \bar{z}_{j}
$$

é uma forma simplética. Note que esta forma é a mesma do exemplo anterior quando fazemos a identificação $\mathbb{C}^{n} \simeq \mathbb{R}^{2 n}$, onde $z_{j}=x_{j}+i y_{j}$.

Vejamos agora um exemplo de uma variedade simplética compacta.

Exemplo 2.4 Seja $M=\mathbb{S}^{2}$, o conjunto de vetores unitários em $\mathbb{R}^{3}$. Note que vetores tangentes a $\mathbb{S}^{2}$ em p podem ser identificados com vetores ortogonais a p. A forma simplética standard em $\mathbb{S}^{2}$ é a sua forma de área dada por

$$
\omega_{p}(u, v)=\langle p, u \times v\rangle, u, v \in T_{p} \mathbb{S}^{2}
$$


Exemplo 2.5 Sejam $\left(M_{1}, \omega_{1}\right)$ e $\left(M_{2}, \omega_{2}\right)$ variedades simpléticas, o produto $M=M_{1} \times M_{2}$ é ainda uma variedade simplética com a forma simplética produto dada por

$$
\omega=p r_{1}^{*} \omega_{1}+p r_{2}^{*} \omega_{2}
$$

onde $\operatorname{pr}_{j}$ são as aplicações projeção

$$
\begin{aligned}
p r_{j}: M_{1} \times M_{2} & \longrightarrow M_{j} \\
\left(p_{1}, p_{2}\right) & \longmapsto p r_{j}\left(p_{1}, p_{2}\right)=p_{j}
\end{aligned}
$$

aqui o símbolo $(*)$ significa o pullback, este define-se da seguinte forma: Dada uma aplicação suave $\varphi: M \rightarrow N$ e $\omega$ uma 2-forma em $N$ defina a 2-forma $\varphi^{*} \omega$ em $M$, chamada o pullback de $\omega$ por $\varphi$, por

$$
\left(\varphi^{*} \omega\right)_{p}(u, v)=\omega_{\varphi(p)}\left(d \varphi_{p}(u), d \varphi_{p}(v)\right), u, v \in T_{p} M
$$

Temos ainda o pullback de funções como segue.

Definição 2.6 Seja $\varphi: M \rightarrow N$ uma aplicação suave entre variedades $M e$ $N$, e suponha que $f: N \rightarrow \mathbb{R}$ é uma função suave em $N$. Então o pullback de $f$ por $\varphi$ é a função suave $\varphi^{*} f$ em $M$ definida por $\left(\varphi^{*} f\right)(x)=f(\varphi(x))$.

Vamos agora classificar variedades simpléticas, para isto definiremos inicialmente simplectomorfismo.

Definição 2.7 Sejam $\left(M_{1}, \omega_{1}\right)$ e $\left(M_{2}, \omega_{2}\right)$ variedades simpléticas de dimensão 2n. Um simplectomorfismo é um difeomorfismo que preserva a estrutura simplética, isto é, $\varphi: M_{1} \rightarrow M_{2}$ difeomorfismo tal que $\varphi^{*} \omega_{2}=\omega_{1}$.

Um dos principais resultados em geometria simplética é o Teorema de Darboux o qual afirma que qualquer variedade simplética de dimensão $2 n$ parece localmente com $\left(\mathbb{R}^{2 n}, \omega_{0}\right)$, isto quer dizer que toda variedade simplética $\left(M^{2 n}, \omega\right)$ é localmente simplectomórfica a $\left(\mathbb{R}^{2 n}, \omega_{0}\right)$.

Teorema 2.8 (Darboux) Sejam $(M, \omega)$ uma variedade simplética de dimensão $2 n$ e $p$ um ponto em $M$. Então existe uma carta coordenada

$$
\left(\mathcal{U}, x_{1}, \ldots, x_{n}, y_{1}, \ldots, y_{n}\right)
$$

centrada em $p$ tal que em $\mathcal{U}$ a forma simplética $\omega$ é

$$
\omega=\sum_{j=1}^{n} d x_{j} \wedge d y_{j} .
$$

Esta carta é chamada carta de Darboux. 
Em particular, uma consequência do Teorema de Darboux é que o único invariante local de variedades simpléticas é a dimensão.

Começaremos agora a introduzir os conceitos de ação de grupos Lie em uma variedade, começamos com a definição de um grupo de Lie.

Definição 2.9 Um grupo de Lie é uma variedade suave G que é também um grupo, com a propriedade que as aplicações multiplicação $m: G \times G \rightarrow G e$ inversão $i: G \rightarrow G$, dadas por

$$
m(g, h)=g h, i(g)=g^{-1},
$$

são ambas suaves.

Grupos de Lie de matrizes e exemplos, são apresentados na Definição 6.2 e no Exemplo 6.3.

Definição 2.10 Se G é um grupo e $S$ é um conjunto, uma ação à esquerda de $G$ em $S$ é uma aplicação

$$
\begin{aligned}
G \times S & \longrightarrow S \\
(g, x) & \longmapsto g \cdot x
\end{aligned}
$$

que satisfaz:

1. regra da associatividade

$$
g_{1} \cdot\left(g_{2} \cdot x\right)=\left(g_{1} \cdot g_{2}\right) \cdot x, \forall g_{1}, g_{2} \in G, \text { e } x \in S
$$

2. $e \cdot x=x, \forall x \in S$.

Observação 2.11 Às vezes é útil dar um nome para uma ação, tal como

$$
\psi: G \times S \longrightarrow S
$$

com a ação de um elemento $g$ do grupo em um ponto $x$, geralmente escrevemos

$$
\psi_{g}(x)
$$

Em termos desta notação, as propriedades (1) e (2) para a ação à esquerda, ficam da seguinte forma:

1. $\psi_{g_{1}} \circ \psi_{g_{2}}=\psi_{g_{1} g_{2}}$

2. $\psi_{e}=I d_{S}$ 
Algumas terminologias importantes

1. Para cada $x \in S$, a órbita de $x$ (ou a órbita de $G$ passando por $x$ ) denotada por $G \cdot x$, é o conjunto de todas as imagens de $x$ sobre a ação por elementos de $G$ :

$$
G \cdot x=\{g \cdot x \mid g \in G\}
$$

2. Para cada $x \in S$, o grupo de isotropia ou estabilizador de $x$, denotado por $G_{x}$, é o conjunto de elementos de $G$ que fixam $x$ :

$$
G_{x}=\{g \in G \mid g \cdot x=x\}
$$

Dizemos que $x$ é um ponto fixado pela ação quando $g \cdot x=x, \forall g \in G$, isto é:

- Órbita: $G \cdot x=\{x\}$

- Estabilizador: $G_{x}=G$

Tendo dado o conceito geométrico de órbita, a seguir enunciamos e demonstramos um resultado que nos diz que duas órbitas são iguais ou disjuntas.

Teorema 2.12 Seja $G$ um grupo agindo em um conjunto $S$. Temos que órbitas diferentes são disjuntas.

Proof. Para mostrarmos a primeira parte usaremos a seguinte estratégia, mostraremos que se duas órbitas se intersectam então elas são iguais. Sejam as órbitas $G \cdot x$ e $G \cdot y$ tal que $G \cdot x \cap G \cdot y \neq \emptyset$, assim, existem $z \in S$ e $g_{1}, g_{2} \in G$ tais que

$$
z=g_{1} \cdot x \text { e } z=g_{2} \cdot y
$$

Seja $u \in G \cdot x$, assim $u=g \cdot x$ para algum $g \in G$, como $x=g_{1}^{-1} \cdot z$, segue que

$$
\begin{aligned}
& u=g \cdot x \\
& =g \cdot\left(g_{1}^{-1} \cdot z\right) \\
& =\left(g \cdot g_{1}^{-1}\right) \cdot\left(g_{2} \cdot y\right) \text { por }(2.2) \\
& =\left(g \cdot g_{1}^{-1} \cdot g_{2}\right) \cdot y \quad \text { associatividade da ação }
\end{aligned}
$$

como $G$ é um grupo, segue que $g \cdot g_{1}^{-1} \cdot g_{2} \in G$, assim $u \in G \cdot y, \operatorname{logo} G \cdot x \subset G \cdot y$.

Analogamente faz-se para $G \cdot y \subset G \cdot x$, assim, concluímos que $G \cdot x=G \cdot y$, e assim está demonstrado o Teorema. 
Definição 2.13 Uma álgebra de Lie (sobre $\mathbb{R}$ ) é um espaço vetorial real $\mathfrak{g}$ dotado com uma aplicação chamado colchete de $\mathfrak{g} \times \mathfrak{g}$ para $\mathfrak{g}$, geralmente denotado por $(X, Y) \mapsto[X, Y]$, que satisfaz as seguintes propriedades para todo $X, Y, Z \in \mathfrak{g}:$

1. Bilinearidade: Para $a, b \in \mathbb{R}$,

$$
\begin{aligned}
& {[a X+b Y, Z]=a[X, Z]+b[Y, Z]} \\
& {[Z, a X+b Y]=a[Z, X]+b[Z, Y]}
\end{aligned}
$$

2. Anti-simetria:

$$
[X, Y]=-[Y, X]
$$

3. Identidade de Jacobi:

$$
[X,[Y, Z]]+[Y,[Z, X]]+[Z,[X, Y]]=0
$$

Exemplo 2.14 $O$ espaço $M(n, \mathbb{R})$ das matrizes $n \times n$ com entradas reais equipada com o colchete comutador

$$
[X, Y]=X Y-Y X
$$

onde $X, Y \in M(n, \mathbb{R})$, é uma álgebra de Lie de dimensão $n^{2}$.

É bem conhecido que $M(n, \mathbb{R})$ é um espaço vetorial de dimensão $n^{2}$, resta-nos verificar apenas as propriedades do colchete, as quais são facilmente verificadas, de fato, sejam $X, Y, Z \in M(n, \mathbb{R})$ e $a, b \in \mathbb{R}$, temos que:

- Bilinearidade

$$
\begin{aligned}
{[a X+b Y, Z] } & =(a X+b Y) Z-Z(a X+b Y) \\
& =a X Z+b Y Z-a Z X-b Z Y \\
& =a(X Z-Z X)+b(Y Z-Z Y) \\
& =a[X, Z]+b[Y, Z]
\end{aligned}
$$

- Anti-simetria

$$
\begin{aligned}
{[X, Y] } & =X Y-Y X \\
& =-(Y X-X Y) \\
& =-[Y, X]
\end{aligned}
$$


- Identidade de Jacobi

$$
\begin{aligned}
& {[X,[Y, Z]]=[X, Y Z-Z Y]=X Y Z-X Z Y-Y Z X+Z Y X} \\
& {[Y,[Z, X]]=[Y, Z X-X Z]=Y Z X-Y X Z-Z X Y+X Z Y} \\
& {[Z,[X, Y]]=[Z, X Y-Y X]=Z X Y-Z Y X-X Y Z+Y X Z}
\end{aligned}
$$

daí

$$
[X,[Y, Z]]+[Y,[Z, X]]+[Z,[X, Y]]=0
$$

portanto temos um exemplo de álgebra de Lie.

Definição 2.15 (Álgebra de Lie de um grupo de Lie) A álgebra de Lie $\mathfrak{g}$ de um grupo de Lie $G$ é o espaço tangente $T_{I} G$ (na identidade) com o colchete de Lie

$$
[X, Y]=X Y-Y X
$$

Denota-se a álgebra de Lie de um particular grupo de Lie usando letras góticas minúsculas.

Uma vez feita a identificação da álgebra de Lie de um grupo de Lie com o espaço tangente ao grupo na identidade, de agora em diante nos referimos a álgebra de Lie de um grupo $G$ apenas por $\mathfrak{g}$.

Exemplo 2.16 Qualquer grupo de Lie $G$ age em si mesmo por conjugação, ou seja

$$
\begin{aligned}
G \times G & \longrightarrow G \\
(g, x) & \longmapsto \psi_{g}(x)=g x g^{-1}
\end{aligned}
$$

A derivada na identidade de

$$
\begin{aligned}
\psi_{g}: G & \longrightarrow G \\
x & \longmapsto \psi_{g}(x)=g x g^{-1}
\end{aligned}
$$

é uma aplicação linear invertível

$$
\operatorname{Ad}_{g}: \mathfrak{g} \longrightarrow \mathfrak{g}
$$

Fazendo $g$ variar, obtemos a representação adjunta (ou ação adjunta) de $G$ em $\mathfrak{g}:$

$$
\begin{aligned}
\operatorname{Ad}: G & \longrightarrow G L(\mathfrak{g}) \\
g & \longmapsto \operatorname{Ad}_{g} .
\end{aligned}
$$

Em outras palavras, a representação adjunta de $G$ em $\mathfrak{g}$ é definida por:

$$
\operatorname{Ad}_{g}(Y)=\left.\frac{d}{d t}\right|_{t=0}\left(g e^{t Y} g^{-1}\right), Y \in \mathfrak{g} .
$$


Seja $\langle\cdot, \cdot\rangle$ o pareamento natural entre $\mathfrak{g}^{*}$ e $\mathfrak{g}$.

$$
\begin{aligned}
\langle\cdot, \cdot\rangle: \mathfrak{g}^{*} \times \mathfrak{g} & \longrightarrow \mathbb{R} \\
(\xi, X) & \longmapsto\langle\xi, X\rangle=\xi(X) .
\end{aligned}
$$

Dado $\xi \in \mathfrak{g}^{*}$, definimos $\operatorname{Ad}_{g}^{*} \xi$ por

$$
\left\langle\operatorname{Ad}_{g}^{*} \xi, X\right\rangle=\left\langle\xi, \operatorname{Ad}_{g^{-1}} X\right\rangle
$$

para algum $X \in \mathfrak{g}$.

A coleção das aplicações $\mathrm{Ad}_{g}^{*}$ formam a representação coadjunta (ou ação coadjunta) de $G$ em $\mathfrak{g}^{*}$ :

$$
\begin{aligned}
\operatorname{Ad}^{*}: G & \longrightarrow G L\left(\mathfrak{g}^{*}\right) \\
g & \longmapsto \operatorname{Ad}_{g}^{*}
\end{aligned}
$$

Definição 2.17 O subconjunto $\mathcal{O}_{\xi}=\left\{\operatorname{Ad}_{g}^{*} \xi \in \mathfrak{g}^{*} \mid g \in G\right\}$ de $\mathfrak{g}^{*}$ é chamado uma órbita coadjunta de $G$ por $\xi \in \mathfrak{g}^{*}$.

Proposição 2.18 Ad e $\mathrm{Ad}^{*}$ satisfazem as seguintes propriedades

1. $\operatorname{Ad}_{g} \circ \operatorname{Ad}_{h}=\operatorname{Ad}_{g h}$

2. $\operatorname{Ad}_{g}^{*} \circ \operatorname{Ad}_{h}^{*}=\operatorname{Ad}_{g h}^{*}$

3. $\operatorname{Ad}_{g}^{-1}=\operatorname{Ad}_{g^{-1}}$

4. $\operatorname{Ad}_{g}[X, Y]=\left[\operatorname{Ad}_{g} X, \operatorname{Ad}_{g} Y\right]$

Observação 2.19 O gerador infinitesimal da ação adjunta de G é o campo vetorial $\xi_{\mathfrak{g}}$ definido por

$$
\xi_{\mathfrak{g}}(\eta)=\left.\frac{d}{d t}\right|_{t=0} \operatorname{Ad}_{\exp (t \xi)} \eta .
$$

Desde que $\mathfrak{g}$ é um espaço vetorial, um campo vetorial em $\mathfrak{g}$ pode ser considerado como uma aplicação de $\mathfrak{g}$ para $\mathfrak{g}$. É possível provar que o gerador infinitesimal da ação adjunta de $G$ é o campo vetorial $\xi_{\mathfrak{g}}$ definido por

$$
\operatorname{ad}_{\xi}(\eta)=\left.\frac{d}{d t}\right|_{t=0} \operatorname{Ad}_{\exp (t \xi)} \eta=\xi_{\mathfrak{g}}(\eta)=[\xi, \eta]
$$

A demonstração deste fato pode ser vista em [15], página 314, Exemplo (a).

A proposição a seguir nos apresenta uma forma explícita da representação adjunta para grupos de Lie de matrizes, esta forma nos será muito útil. 
Proposição 2.20 Se G é um grupo de Lie de matrizes, então

$$
\operatorname{Ad}_{g}(Y)=g Y g^{-1}, \forall g \in G, \forall Y \in \mathfrak{g}
$$

Proof. Sabemos que

$$
\operatorname{Ad}_{g}(Y)=\left.\frac{d}{d t}\right|_{t=0}\left(g \exp (t Y) g^{-1}\right)
$$

assim

$$
\operatorname{Ad}_{g}(Y)=\left.\frac{d}{d t}\right|_{t=0} g \cdot\left(I+t Y+\frac{t^{2} Y^{2}}{2 !}+\cdots\right) \cdot g^{-1}=g Y g^{-1}
$$

Proposição 2.21 Para grupos de Lie de matrizes tem-se que $\operatorname{ad}_{Y} X=[Y, X]$, $Y, X \in \mathfrak{g}$.

Proof. Sabemos da proposição anterior que se $G$ é um grupo de Lie de matrizes, então

$$
\operatorname{Ad}_{g} X=g X g^{-1}, g \in, X \in \mathfrak{g} .
$$

Mostraremos que

$$
\operatorname{ad}_{Y} X=[Y, X], Y, X \in \mathfrak{g}
$$

para isto, calculamos

$$
\begin{aligned}
\operatorname{ad}_{Y} X & =\left.\frac{d}{d t}\right|_{t=0} \operatorname{Ad}_{\exp (t Y)} X \\
& =\left.\frac{d}{d t}\right|_{t=0} \exp (t Y) X \exp (-t Y) \\
& =\left.\frac{d}{d t}\right|_{t=0}\left(I+t Y+\frac{t^{2} Y}{2}+\cdots\right) X\left(I-t Y+\frac{t^{2} Y}{2}-\cdots\right) \\
& =Y X I-I X Y \\
& =Y X-X Y \\
& =[Y, X]
\end{aligned}
$$

portanto $\operatorname{ad}_{Y} X=[Y, X]$

No próximo resultado, estudaremos o espaço tangente em um ponto $\xi$ de um órbita coadjunta, usaremos a identificação de que o espaço tangente a um grupo de Lie $G$ na identidade é identificado com a álgebra de Lie $\mathfrak{g}$ desse grupo. 
Proposição 2.22 Seja $\mathcal{O}$ uma órbita da ação coadjunta de um grupo de Lie $G$ em $\mathfrak{g}^{*}$. Se $\xi \in \mathcal{O}$ então

$$
T_{\xi} \mathcal{O}=\left\{\operatorname{ad}_{X}^{*}(\xi) \mid X \in \mathfrak{g}\right\}
$$

Proof. Precisamos descrever os vetores tangentes à órbita coadjunta $\mathcal{O}$ no ponto $\xi$, para isto, sejam $X \in \mathfrak{g}$ e $g(t)$ uma curva em $G$ cujo vetor tangente em $g(0)$ é $X$.

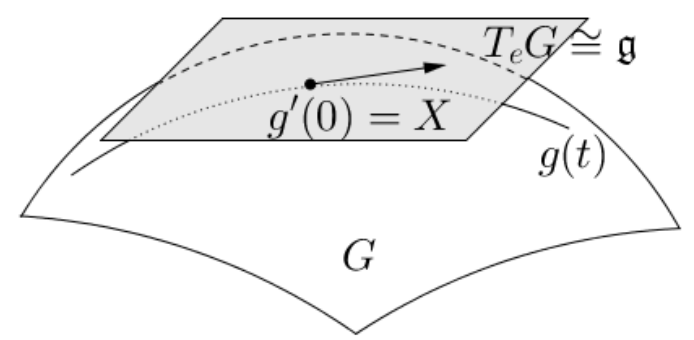

Figura 2.1: Álgebra de Lie identificado com o espaço tangente

Por exemplo, podemos tomar $g(t)=\exp (t X)$ e $\xi \in \mathcal{O}$. Sejam $Y \in \mathfrak{g}$ e $\xi(t)=\operatorname{Ad}_{g(t)}^{*} \xi$ uma curva em $\mathcal{O} \operatorname{com} \xi(0)=\xi$. Note que temos a identidade

$$
\langle\xi(t), Y\rangle=\left\langle\operatorname{Ad}_{g(t)}^{*} \xi, Y\right\rangle
$$

de (2.3) obtemos que

$$
\langle\xi(t), Y\rangle=\left\langle\xi, \operatorname{Ad}_{g(t)^{-1}} Y\right\rangle
$$

diferenciando esta última com respeito a $t$ em $t=0$, obtemos

$$
\left\langle\xi^{\prime}(0), Y\right\rangle=\left\langle\xi,-\operatorname{ad}_{X} Y\right\rangle
$$

dessa forma

$$
\left\langle\xi^{\prime}(0), Y\right\rangle=\left\langle\operatorname{ad}_{X}^{*} \xi, Y\right\rangle
$$

assim, $\xi^{\prime}(0)=\operatorname{ad}_{X}^{*} \xi$. Portanto $T_{\xi} \mathcal{O}=\left\{\operatorname{ad}_{X}^{*}(\xi) \mid X \in \mathfrak{g}\right\}$.

O resultado a seguir mostra que toda órbita coadjunta carrega uma estrutura simplética natural, o que a torna uma variedade simplética.

Teorema 2.23 Seja $G$ um grupo de Lie e $\mathrm{Ad}^{*}: G \times \mathfrak{g}^{*} \longrightarrow \mathfrak{g}^{*}$ sua ação coadjunta. Seja $\mathcal{O} \subset \mathfrak{g}^{*}$ uma órbita coadjunta. Então

$$
\omega_{\xi}^{ \pm}\left(\operatorname{ad}_{\mu}^{*}(\xi), \operatorname{ad}_{\eta}^{*}(\xi)\right)= \pm\langle\xi,[\mu, \eta]\rangle
$$

para todo $\xi \in \mathcal{O}$ e $\mu, \eta \in \mathfrak{g}$ define formas simplética em $\mathcal{O}$. Nos referimos a $\omega^{ \pm}$como as estruturas simpléticas na órbita coadjunta, a forma $\omega^{ \pm} e ́$ denominda a forma de Kostant-Kirillov-Souriau. Além disso, a restrição da 
ação coadjunta $\left.\mathrm{Ad}^{*}\right|_{G \times \mathcal{O}}$ é Hamiltoniana com aplicação momento dada pela inclusão $i_{\mathcal{O}}: \mathcal{O} \rightarrow \mathfrak{g}^{*}$.

Proof. Aqui nos limitaremos a demonstrar que $\omega_{\xi}$ é de fato uma forma simplética, para as demais informações deste teorema o prezado leitor pode consultar [8], página 212, Exemplo 5.3.11. Nas contas que seguem usaremos a notação

$$
\eta_{*}(\xi)=\left.\frac{d}{d t} \operatorname{Ad}_{\exp (t \eta)}^{*} \xi\right|_{t=0} .
$$

Afim de que $\omega_{\xi}$ seja simplética é necessário provar que $\omega_{\xi}$

- é bilinear;

Temos que

$$
\left(k_{1} \eta^{1}+k_{2} \eta^{2}\right)_{*}(\xi)=k_{1} \eta_{*}^{1}(\xi)+k_{2} \eta_{*}^{2}(\xi) \text {, onde } k_{1}, k_{2} \in \mathbb{R}, \eta^{1}, \eta^{2} \in \mathfrak{g}
$$

dessa forma

$$
\begin{aligned}
\omega_{\xi}\left(k_{1} \eta_{*}^{1}(\xi)+k_{2} \eta_{*}^{2}(\xi), \eta_{*}^{3}(\xi)\right) & =\omega_{\xi}\left(\left(k_{1} \eta^{1}+k_{2} \eta^{2}\right)_{*}(\xi), \eta_{*}^{3}(\xi)\right) \\
& =\left\langle\xi,\left[k_{1} \eta^{1}+k_{2} \eta^{2}, \eta^{3}\right]\right\rangle \\
& =k_{1}\left\langle\xi,\left[\eta^{1}, \eta^{3}\right]\right\rangle+k_{2}\left\langle\xi,\left[\eta^{2}, \eta^{3}\right]\right\rangle \\
& =k_{1} \omega_{\xi}\left(\eta_{*}^{1}(\xi), \eta_{*}^{3}(\xi)\right)+k_{2} \omega_{\xi}\left(\eta_{*}^{2}(\xi), \eta_{*}^{3}(\xi)\right)
\end{aligned}
$$

portanto é bilinear.

- é anti-simétrica;

A anti-simetria vem do fato do colchete de Lie ser anti-simétrico, de fato

$$
\omega_{\xi}\left(\eta_{*}^{1}(\xi), \eta_{*}^{2}(\xi)\right)=\left\langle\xi,\left[\eta^{1}, \eta^{2}\right]\right\rangle=-\left\langle\xi,\left[\eta^{2}, \eta^{1}\right]\right\rangle=-\omega_{\xi}\left(\eta_{*}^{2}(\xi), \eta_{*}^{1}(\xi)\right)
$$

- é não-degenerada;

Suponha que

$$
\omega_{\xi}\left(\eta_{*}^{1}(\xi), \eta_{*}^{2}(\xi)\right)=0, \forall \eta^{2} \in \mathfrak{g}
$$

então,

$$
0=\omega_{\xi}\left(\eta_{*}^{1}(\xi), \eta_{*}^{2}(\xi)\right)=\left\langle\xi,\left[\eta^{1}, \eta^{2}\right]\right\rangle=\left\langle\xi, \operatorname{ad}_{\eta^{1}} \eta^{2}\right\rangle=\left\langle\operatorname{ad}_{\eta^{1}}^{*} \xi, \eta^{2}\right\rangle
$$

como vale para todo $\eta^{2} \in \mathfrak{g}$, segue que $\eta_{*}^{1}(\xi)=\operatorname{ad}_{\eta^{1}}^{*} \xi=0$ (a menos de sinal, o que não faz diferença), portanto $\omega_{\xi}$ é não-degenerada.

- é fechada.

Para esta parte usaremos o seguinte resultado: 
Proposição 2.24 Para $\alpha \in \Omega^{k}(M)$ e $X_{0}, \ldots, X_{k} \in \mathfrak{X}(M)$, temos que

$$
\begin{aligned}
d \alpha\left(X_{0}, \ldots, X_{k}\right)= & \sum_{i=0}^{k}(-1)^{i} X_{i}\left(\alpha\left(X_{0}, \ldots, \hat{X}_{i}, \ldots, X_{k}\right)\right)+ \\
& \sum_{i<j}(-1)^{i+j} \alpha\left(\left[X_{i}, X_{j}\right], X_{0}, \ldots, \hat{X}_{i}, \ldots, \hat{X}_{j}, \ldots, X_{k}\right)
\end{aligned}
$$

onde $\hat{X}_{i}$ significa que $X_{i}$ foi omitido.

A demonstração desta proposição o prezado leitor pode consultar [19] página 170. Um outro resultado que usaremos é

Teorema 2.25 Para uma $k$-forma suave $\omega$ e campos vetoriais suaves $X, Y_{1}, \ldots, Y_{k}$ em uma variedade $M$, temos:

$$
\left(\mathcal{L}_{X} \omega\right)\left(Y_{1}, \ldots, Y_{k}\right)=X\left(\omega\left(Y_{1}, \ldots, Y_{k}\right)\right)-\sum_{i=1}^{k} \omega\left(Y_{1}, \ldots,\left[X, Y_{i}\right], \ldots, Y_{k}\right)
$$

A demonstração deste teorema o prezado leitor pode consultar [24], página 232. Afim de não carregar a notação, usaremos apenas $\eta_{*}$ para denotar $\eta_{*}(\xi)$. Sejam $A, B, C \in \mathfrak{g}$ e $A_{*}, B_{*}, C_{*}$ como em (2.7), devido a $G$-invariância de $\omega_{\xi}$, a qual será provada no Teorema 2.27 , segue que

$$
\mathcal{L}_{A_{*}} \omega=\mathcal{L}_{B_{*}} \omega=\mathcal{L}_{C_{*}} \omega=0
$$

do Teorema 2.25 temos

$$
\left(\mathcal{L}_{A_{*}} \omega\right)\left(B_{*}, C_{*}\right)=A_{*}\left(\omega\left(B_{*}, C_{*}\right)\right)-\omega\left(\left[A_{*}, B_{*}\right], C_{*}\right)-\omega\left(B_{*},\left[A_{*}, C_{*}\right]\right)
$$

dessa forma, obtemos que

$$
\begin{aligned}
& A_{*}\left(\omega\left(B_{*}, C_{*}\right)\right)=\omega\left(\left[A_{*}, B_{*}\right], C_{*}\right)+\omega\left(B_{*},\left[A_{*}, C_{*}\right]\right) \\
& B_{*}\left(\omega\left(A_{*}, C_{*}\right)\right)=\omega\left(\left[B_{*}, A_{*}\right], C_{*}\right)+\omega\left(A_{*},\left[B_{*}, C_{*}\right]\right) \\
& C_{*}\left(\omega\left(A_{*}, B_{*}\right)\right)=\omega\left(\left[C_{*}, A_{*}\right], B_{*}\right)+\omega\left(A_{*},\left[C_{*}, B_{*}\right]\right) .
\end{aligned}
$$

Dessa forma, usando estas três igualdades e a Proposição 2.24, obtemos 
que

$$
\begin{aligned}
\mathrm{d} \omega\left(A_{*}, B_{*}, C_{*}\right)= & A_{*}\left(\omega\left(B_{*}, C_{*}\right)\right)-B_{*}\left(\omega\left(A_{*}, C_{*}\right)\right)+C_{*}\left(\omega\left(A_{*}, B_{*}\right)\right)- \\
& \omega\left(\left[A_{*}, B_{*}\right], C_{*}\right)+\omega\left(\left[A_{*}, C_{*}\right], B_{*}\right)-\omega\left(\left[B_{*}, C_{*}\right], A_{*}\right) \\
= & \omega\left(\left[A_{*}, B_{*}\right], C_{*}\right)+\omega\left(B_{*},\left[A_{*}, C_{*}\right]\right)-\omega\left(\left[B_{*}, A_{*}\right], C_{*}\right)- \\
& \omega\left(A_{*},\left[B_{*}, C_{*}\right]\right)+\omega\left(\left[C_{*}, A_{*}\right], B_{*}\right)+\omega\left(A_{*},\left[C_{*}, B_{*}\right]\right)- \\
& \omega\left(\left[A_{*}, B_{*}\right], C_{*}\right)+\omega\left(\left[A_{*}, C_{*}\right], B_{*}\right)-\omega\left(\left[B_{*}, C_{*}\right], A_{*}\right) \\
= & \omega\left(\left[A_{*}, B_{*}\right], C_{*}\right)+\omega\left(B_{*},\left[A_{*}, C_{*}\right]\right)+\omega\left(A_{*},\left[C_{*}, B_{*}\right]\right) \\
= & \langle\xi,[[A, B], C]\rangle+\langle\xi,[B,[A, C]]\rangle+\langle\xi,[A,[C, B]]\rangle \\
= & \langle\xi,[C,[B, A]]+[B,[A, C]]+[A,[C, B]]\rangle \\
= & 0
\end{aligned}
$$

portanto $\omega_{\xi}$ é fechada, note que usamos a definição da forma $\omega$ e a identidade de Jacobi para o colchete de Lie. Concluímos assim que $\omega_{\xi}$ é uma forma simplética na órbita coadjunta.

Dessa forma, as órbitas coadjuvantes são variedades simpléticas e portanto temos o seguinte

Corolário 2.26 Órbitas coadjunta de grupos de Lie de dimensão finita são de dimensão par e orientáveis.

Provaremos agora o resultado utilizado na demonstração anterior.

Teorema 2.27 A forma simplética na órbita coadjunta é G-invariante.

Proof. A forma é dada por

$$
\omega_{\xi}\left(X_{*}, Y_{*}\right)=\omega_{\xi}\left(\operatorname{ad}_{X}^{*} \xi, \operatorname{ad}_{Y}^{*} \xi\right):=\langle\xi,[X, Y]\rangle, X, Y \in \mathfrak{g}, \xi \in \mathcal{O}
$$

pois

$$
X_{*}(\xi)=\left.\frac{d}{d t}\right|_{t=0} \operatorname{Ad}_{\exp (t X)}^{*} \xi=\operatorname{ad}_{X}^{*} \xi
$$

na demonstração usaremos o fato que diz que se $\psi$ denota a ação de um grupo de Lie $G$ então

$$
\psi_{a *} X_{*}=\left(\operatorname{Ad}_{a} X\right)_{*}
$$

usaremos também que o pullback é dado por

$$
\left(\varphi^{*} \omega\right)_{p}(u, v)=\omega_{\varphi(p)}\left(d \varphi_{p}(u), d \varphi_{p}(v)\right), u, v \in T_{p} M
$$


assim,

$$
\begin{aligned}
\left(\left(\operatorname{Ad}_{a}^{*}\right)^{*} \omega\right)_{\xi}\left(X_{*}, Y_{*}\right) & =\omega_{\operatorname{Ad}_{a}^{*} \xi}\left(\left(\operatorname{Ad}_{a}^{*}\right)_{*} X_{*},\left(\operatorname{Ad}_{a}^{*}\right)_{*} Y_{*}\right) \\
& =\omega_{\operatorname{Ad}_{a}^{*} \xi}\left(\left(\operatorname{Ad}_{a} X\right)_{*},\left(\operatorname{Ad}_{a} Y\right)_{*}\right) \\
& =\left\langle\operatorname{Ad}_{a}^{*} \xi,\left[\operatorname{Ad}_{a} X, \operatorname{Ad}_{a} Y\right]\right\rangle \\
& =\left\langle\xi, \operatorname{Ad}_{a^{-1}} \operatorname{Ad}_{a}[X, Y]\right\rangle \\
& =\langle\xi,[X, Y]\rangle \\
& =\omega_{\xi}\left(X_{*}, Y_{*}\right)
\end{aligned}
$$




\section{Espaço de moduli de polígonos em $\mathbb{R}^{3}$.}

Um $n$-gon $P$ (polígono de $n$ lados) em $\mathbb{R}^{3}$ é determinado por seus $n$ vértices $p_{1}, \ldots, p_{n}$ unidos pelos segmentos orientados $v_{j}=p_{j+1}-p_{j}, 1 \leq j \leq$ $n-1$ e $v_{n}=p_{1}-p_{n}$. Um polígono é dito ser degenerado se estiver em uma linha. Seja $\mathcal{P}_{n}$ o espaço dos $n$-gons em $\mathbb{R}^{3}$. Dois polígonos $P=\left(p_{1}, \ldots p_{n}\right)$ e $Q=\left(q_{1}, \ldots, q_{n}\right)$ são identificados se existe uma isometria $g$ de $\mathbb{R}^{3}$ preservando a orientação que envia os vértices de $P$ para os vértices de $Q$, isto é

$$
g p_{i}=q_{i}, 1 \leq i \leq n .
$$

Seja $r=\left(r_{1}, \ldots, r_{n}\right) \in \mathbb{R}_{+}^{n}$, o espaço de moduli de polígonos $M_{r}$ em $\mathbb{R}^{3}$ é definido como sendo o espaço dos $n$-gons com lados de comprimento fixados $r_{1}, \ldots, r_{n}$ respectivamente módulo isometrias como acima. Este espaço de moduli de polígonos em $\mathbb{R}^{3}$ pode ser realizado como um quociente simplético, como segue sem muitos detalhes.

Sejam $r=\left(r_{1}, \ldots, r_{n}\right) \in \mathbb{R}_{+}^{n}$ e o produto

$$
\mathcal{S}_{r}=\prod_{j=1}^{n} \mathbb{S}_{r_{j}}^{2}
$$

onde $\mathbb{S}_{r_{j}}^{2}$ são esferas em $\mathbb{R}^{3}$ de raio $r_{j}$ e centro na origem, note que $\mathcal{S}_{r}$ é uma variedade suave pois é produto de variedades suaves. Considere

$$
\operatorname{pr}_{j}: \mathcal{S}_{r} \rightarrow \mathbb{S}_{r_{j}}^{2}
$$

a projeção no $j$-ésimo fator e $\omega_{j}$ a forma de volume na esfera $\mathbb{S}_{r_{j}}^{2}$. Uma vez que a forma $\omega_{j}$ é fechada e não degenerada para todo $j$, a 2 -forma

$$
\omega=\sum_{j=1}^{n} \frac{1}{r_{j}} \operatorname{pr}_{j}^{*} \omega_{j}
$$

é fechada e não degenerada e define uma estrutura simplética em $\mathcal{S}_{r}$.

Note que podemos identificar cada esfera $\mathbb{S}_{r_{j}}^{2}$ com uma órbita coadjunta de $S O(3)$, e em cada órbita considerar a ação de $S O(3)$ sendo a ação coadjunta, ou seja, $S O(3)$ age diagonalmente em $\mathcal{S}_{r}$. Pelo exposto na seção 8.1, referente 
a forma de Killing, sabemos que $S O(3)$ é um grupo de Lie semisimples, dessa forma podemos escolher um produto interno invariante na álgebra de Lie $\mathfrak{s o}(3)$ de $S O(3)$, assim teremos a identificação $\mathfrak{s o}^{*}(3) \simeq \mathbb{R}^{3}$. Em cada esfera $\mathbb{S}_{r_{j}}^{2}$ o mapa momento associado a ação coadjunta é a inclusão de $\mathbb{S}_{r_{j}}^{2}$ em $\mathbb{R}^{3}$, por linearidade, a ação diagonal de $S O(3)$ em $\mathcal{S}_{r}$ tem mapa momento

$$
\begin{aligned}
\mu: \mathcal{S}_{r} & \longrightarrow \mathbb{R}^{3} \\
\vec{v}=\left(v_{1}, \ldots, v_{n}\right) & \longmapsto v_{1}+\cdots+v_{n} .
\end{aligned}
$$

O conjunto de nível

$$
\mu^{-1}(0):=\widetilde{M}_{r}=\left\{\vec{v}=\left(v_{1}, \ldots, v_{n}\right) \in \mathcal{S}_{r} \mid \sum_{j=1}^{n} v_{j}=0\right\}
$$

é uma subvariedade de $\mathcal{S}_{r}$ pois 0 é um valor regular para $\mu$.

Um polígono em $\mathbb{R}^{3}$ é um caminho linear por parte, fechado em $\mathbb{R}^{3}$. Considere o caminho linear por parte tal que o $j$-ésimo passo é dado pelo vetor $v_{j}$. Tal caminho fecha se, e somente se, $\sum_{j=1}^{n} v_{j}=0$. Portanto, $\widetilde{M}_{r}$ é o espaço $\mathcal{P}_{n}$ dos $n$-gons de lados de comprimento fixados $r_{1}, \ldots, r_{n}$. A existência de uma isometria $g$ tal que $g p_{i}=q_{i}$ é equivalente a existência de $A \in S O(3)$ tal que $A \vec{v}=\vec{u}$, onde $\vec{u}=\left(u_{1}, \ldots, u_{n}\right), u_{j}=q_{j+1}-q_{j}, 1 \leq j \leq n-1, u_{n}=q_{1}-q_{n}$. Assim o quociente topológico $\widetilde{M}_{r} / S O(3)$ é o espaço de moduli $M_{r}$ dos $n$-gos de lados de comprimento fixado descrito acima e $M_{r}$ é realizado como o quociente simplético

$$
M_{r}:=\widetilde{M}_{r} / S O(3)=\mathcal{S}_{r} / / S O(3)
$$

O espaço de moduli $M_{r}$ é uma variedade suave se, e somente se, o vetor de comprimento $r$ é genérico, isto é, para cada $I \subset\{1, \ldots, n\}$ a quantidade

$$
\epsilon_{I}(r):=\sum_{j \in I} r_{j}-\sum_{j \in I^{c}} r_{j}
$$

é não nulo. Equivalentemente, se, e somente se, em $M_{r}$ não existe polígonos degenerados. De fato, se existe um polígono $P$ em uma linha (ou um conjunto de índices $I$ tal que $\epsilon_{I}(r)=0$ ) então seu estabilizador é $\mathbb{S}^{1}$ desde que o polígono $P$ é fixado por rotações em torno do eixo que define. Portanto a ação de $S O(3)$ em $\widetilde{M}_{r}$ não é livre e o quociente tem sigularidades as quais correspondem aos $n$-gons degenerados em $M_{r}$. 


\section{4}

\section{Variedades de Poisson}

Nosso intuito é fornecer uma breve introdução a Geometria de Poisson. Apresentamos os principais conceitos, dentre eles o colchete e variedade de Poisson, mostramos que as variedades simpléticas são um caso particular de variedade de Poisson. Exibimos alguns exemplos, dentre eles a estrutura de Poisson em $\mathfrak{g}^{*}$ e na órbita coadjunta $\mathcal{O} \subset \mathfrak{g}^{*}$, apresentamos o resultado que diz que as folhas simpléticas em $\mathfrak{g}^{*}$ coincidem com as órbitas da ação coadjunta e provamos que a ação coadjunta diagonal é uma ação de Poisson.

Definição 4.1 Um colchete de Poisson em uma variedade $M$ é uma operação binária $\mathcal{C}^{\infty}(M) \times \mathcal{C}^{\infty}(M) \rightarrow \mathcal{C}^{\infty}(M),(f, g) \mapsto\{f, g\}$, satisfazendo:

1. Anti-simetria: $\{f, g\}=-\{g, f\}$;

2. $\mathbb{R}$-bilinearidade: $\{f, a g+b h\}=a\{f, g\}+b\{f, h\}$ onde $a, b \in \mathbb{R}$;

3. Identidade de Jacobi: $\{f,\{g, h\}\}+\{g,\{h, f\}\}+\{h,\{f, g\}\}=0$;

4. Regra de Leibniz: $\{f, g \cdot h\}=g \cdot\{f, h\}+\{f, g\} \cdot h$.

O par $(M,\{\cdot, \cdot\})$ é chamado de variedade de Poisson

Em outras palavras, $\mathcal{C}^{\infty}(M)$ equipada $\operatorname{com}\{\cdot, \cdot\}$ é uma álgebra de Lie cujo colchete de Lie satisfaz a regra de Leibniz.

Para $X \in \mathfrak{X}(M)$ e $\omega \in \Omega^{2}(M)$, o produto interno de $X$ e $\omega$, denotado por $i_{X} \omega \in \Omega^{1}(M)$ é definido como

$$
\left(i_{X} \omega\right)(Y)=\omega(X, Y) \text { para } Y \in \mathfrak{X}(M)
$$

Definição $4.2 \operatorname{Seja}(M, \omega)$ uma variedade simplética e $H \in \mathcal{C}^{\infty}(M)$. Um campo vetorial $X_{H}$ em $M$ é chamado campo vetorial Hamiltoniano com a função energia $H$, se para $X_{H}$ temos

$$
i_{X_{H}} \omega=d H
$$

Para $X \in \mathfrak{X}(M)$ e $f \in \mathcal{C}^{\infty}(M)$, definimos a derivada de Lie de uma função, por

$$
\mathcal{L}_{X} f(p)=\mathrm{d} f_{p}\left(X_{p}\right), p \in M
$$


Proposição 4.3 Sejam $f \in \mathcal{C}^{\infty}(M), X, Y \in \mathfrak{X}(M)$, a derivada de Lie nos fornece
1. $\mathcal{L}_{X} f=X(f)$
2. $\mathcal{L}_{X} Y=[X, Y]$

O prezado leitor pode encontrar as demonstrações destas duas igualdades em [24], nas páginas 225 e 226.

Podemos definir em $(M, \omega)$ um colchete natural, chamado o colchete de Poisson de $\omega$, como segue, sejam $f, g \in \mathcal{C}^{\infty}(M)$

$$
\{f, g\}=\omega\left(X_{f}, X_{g}\right)=\left(i_{X_{f}} \omega\right)\left(X_{g}\right)=\mathrm{d} f\left(X_{g}\right)=-X_{g}(f)=X_{f}(g)
$$

Iremos mostrar na Proposição 4.7 que de fato o colchete acima é Poisson. Também é possível definir esse colchete usando a derivada de Lie, e isto é feito no próximo resultado.

Proposição 4.4 Seja $(M, \omega)$ uma variedade simplética. Para $f, g \in \mathcal{C}^{\infty}(M)$ temos

$$
\{f, g\}=-\mathcal{L}_{X_{f}} g=\mathcal{L}_{X_{g}} f
$$

Proof. Considere o campo vetorial Hamiltoniano $X_{g}$, sabemos da Definição 4.2 que

$$
i_{X_{g}} \omega=\mathrm{d} g
$$

e pela definição da derivada de Lie de uma função apresentada em (4.1), teremos

$$
\mathcal{L}_{X_{f}} g=\mathrm{d} g\left(X_{f}\right)=\left(i_{X_{g}} \omega\right)\left(X_{f}\right)=\omega\left(X_{g}, X_{f}\right)=-\omega\left(X_{f}, X_{g}\right)=-\mathcal{L}_{X_{g}} f
$$

Corolário 4.5 Seja $(M, \omega)$ uma variedade simplética. Para $f \in \mathcal{C}^{\infty}(M)$, $g \mapsto\{f, g\}$ é uma derivação.

Proof. Para $g, h \in \mathcal{C}^{\infty}(M)$ temos

$$
\begin{aligned}
& \{f, g \cdot h\}=-\mathcal{L}_{X_{f}}(g \cdot h) \quad \text { Proposição } 4.4 \\
& =-\mathrm{d}(g \cdot h)\left(X_{f}\right) \\
& =-((\mathrm{d} g) \cdot h+g \cdot(\mathrm{d} h))\left(X_{f}\right) \text { derivada do produto } \\
& =-h \mathrm{~d} g\left(X_{f}\right)-g \mathrm{~d} h\left(X_{f}\right) \\
& =-h \mathcal{L}_{X_{f}} g-g \mathcal{L}_{X_{f}} h \\
& =\{f, g\} \cdot h+g \cdot\{f, h\}
\end{aligned}
$$


Para definir o campo vetorial Hamiltoniano de uma função, o que realmente precisa não é uma estrutura simplética, mas uma estrutura de Poisson: A identidade de Leibniz significa que, para uma dada função $f$ em uma variedade de Poisson $M$, a aplicação $g \mapsto\{f, g\}$ é uma derivação. Assim, existe um único campo vetorial $X_{f}$ em $M$, chamado o campo vetorial Hamiltoniano de $f$, tal que para qualquer $g \in \mathcal{C}^{\infty}(M)$ temos

$$
X_{f}(g)=\{f, g\}
$$

Daí a definição

Definição 4.6 Sejam $(M,\{\cdot, \cdot\})$ uma variedade de Poisson e $h \in \mathcal{C}^{\infty}(M)$. O campo vetorial $X_{h}=\{h, \cdot\}$ é chamado o campo vetorial Hamiltoniano associado a função Hamiltoniana $h$ e escrevemos

$$
\operatorname{Ham}(M,\{\cdot, \cdot\}):=\left\{X_{h} \mid h \in \mathcal{C}^{\infty}(M)\right\}
$$

para o espaço vetorial dos campos vetoriais Hamiltonianos. Uma função $h \in$ $\mathcal{C}^{\infty}(M)$ cujo campo vetorial Hamiltoniano é zero, $X_{h}=0$, é chamada uma função de Casimir ou uma Casimir e denotamos por

$$
\operatorname{Cas}(M,\{\cdot, \cdot\}):=\left\{h \in \mathcal{C}^{\infty}(M) \mid X_{h}=0\right\}
$$

para o espaço (vetorial) das funções de Casimir.

Na proposição a seguir usaremos a seguinte identidade

$$
\omega\left(X_{f},\left[X_{g}, X_{h}\right]\right)=\left[X_{g}, X_{h}\right](f)
$$

a qual é facilmente demonstrada como abaixo

$$
\begin{aligned}
\omega\left(X_{f},\left[X_{g}, X_{h}\right]\right) & =\left(i_{X_{f}} \omega\right)\left(\left[X_{g}, X_{h}\right]\right) \\
& =\mathrm{d} f\left(\left[X_{g}, X_{h}\right]\right) \\
& =\mathcal{L}_{\left[X_{g}, X_{h}\right]} f \\
& =\left[X_{g}, X_{h}\right](f)
\end{aligned}
$$

O resultado a seguir nos diz que toda variedade simplética pode ser vista como uma variedade de Poisson.

Proposição 4.7 Se $(M, \omega)$ é uma variedade simplética suave, então o colchete $\{f, g\}=\omega\left(X_{f}, X_{g}\right)$ é uma estrutura de Poisson em $M$. 
Proof. Para mostrarmos que $\{f, g\}=\omega\left(X_{f}, X_{g}\right)$ de fato define um colchete de Poisson em $M$, precisamos verificar os itens da Definição 4.1, para isto, sejam $f, g, h \in \mathcal{C}^{\infty}(M)$ e $a, b \in \mathbb{R}$

1. Anti-simetria

$$
\{f, g\}=\omega\left(X_{f}, X_{g}\right)=-\omega\left(X_{g}, X_{f}\right)=-\{g, f\}
$$

pois $\omega$ é anti-simética.

2. $\mathbb{R}$-bilinearidade

$$
\begin{aligned}
\{f, a g+b h\} & =-\{a g+b h, f\} \\
& =-\omega\left(X_{a g+b h}, X_{f}\right) \\
& =-\left(i_{X_{a g+b h}} \omega\right)\left(X_{f}\right) \\
& =-\mathrm{d}(a g+b h)\left(X_{f}\right) \\
& =-\left(a \mathrm{~d} g\left(X_{f}\right)+b \mathrm{~d} h\left(X_{f}\right)\right) \\
& =-\left(a i_{X_{g}} \omega\left(X_{f}\right)+b i_{X_{h}} \omega\left(X_{f}\right)\right) \\
& =-\left(a \omega\left(X_{g}, X_{f}\right)+b \omega\left(X_{h}, X_{f}\right)\right) \\
& =a \omega\left(X_{f}, X_{g}\right)+b \omega\left(X_{f}, X_{h}\right) \\
& =a\{f, g\}+b\{f, h\}
\end{aligned}
$$

3. Identidade de Jacobi. Nesta usaremos o resultado da Proposição 2.24, a identidade (4.2) e o fato de que $\mathrm{d} \omega=0$.

Sejam $X_{f}, X_{g}$ e $X_{h}$ campos vetoriais Hamiltonianos, assim

$$
\begin{aligned}
0= & \mathrm{d} \omega\left(X_{f}, X_{g}, X_{h}\right) \\
= & X_{f}\left(\omega\left(X_{g}, X_{h}\right)\right)-X_{g}\left(\omega\left(X_{f}, X_{h}\right)\right)+X_{h}\left(\omega\left(X_{f}, X_{g}\right)\right) \\
& -\omega\left(\left[X_{f}, X_{g}\right], X_{h}\right)+\omega\left(\left[X_{f}, X_{h}\right], X_{g}\right)-\omega\left(\left[X_{g}, X_{h}\right], X_{f}\right) \\
= & X_{f}\left(\omega\left(X_{g}, X_{h}\right)\right)+X_{g}\left(\omega\left(X_{h}, X_{f}\right)\right)+X_{h}\left(\omega\left(X_{f}, X_{g}\right)\right) \\
& +\omega\left(X_{h},\left[X_{f}, X_{g}\right]\right)+\omega\left(X_{g},\left[X_{h}, X_{f}\right]\right)+\omega\left(X_{f},\left[X_{g}, X_{h}\right]\right) \\
= & X_{f}(\{g, h\})+X_{g}(\{h, f\})+X_{h}(\{f, g\})+\left[X_{f}, X_{g}\right](h)+\left[X_{h}, X_{f}\right](g) \\
& +\left[X_{g}, X_{h}\right](f) \\
= & \{f,\{g, h\}\}+\{g,\{h, f\}\}+\{h,\{f, g\}\}+X_{f}\left(X_{g}(h)\right)-X_{g}\left(X_{f}(h)\right) \\
& +X_{h}\left(X_{f}(g)\right)-X_{f}\left(X_{h}(g)\right)+X_{g}\left(X_{h}(f)\right)-X_{h}\left(X_{g}(f)\right) \\
= & \{f,\{g, h\}\}+\{g,\{h, f\}\}+\{h,\{f, g\}\}+\{f,\{g, h\}\}-\{g,\{f, h\}\} \\
& +\{h,\{f, g\}\}-\{f,\{h, g\}\}+\{g,\{h, f\}\}-\{h,\{g, f\}\} \\
= & 3(\{f,\{g, h\}\}+\{g,\{h, f\}\}+\{h,\{f, g\}\})
\end{aligned}
$$

logo a identidade de Jacobi está demonstrada.

4. A regra de Leibniz foi provada no corolário 4.5. 
Dessa forma concluímos que $\{f, g\}=\omega\left(X_{f}, X_{g}\right)$ é uma estrutura de Poisson em $M$. Assim, qualquer variedade simplética é também uma variedade de Poisson, no entanto, a recíproca não é verdadeira, uma das formas de justificar isto é o fato de que toda variedade simplética tem dimensão par, enquanto que as variedades de Poisson não têm essa restrição.

Proposição 4.8 Para quaisquer $f, g \in \mathcal{C}^{\infty}(M)$, vale

$$
X_{\{f, g\}}=\left[X_{f}, X_{g}\right]
$$

Proof. Para quaisquer $f, g, h \in \mathcal{C}^{\infty}(M)$ temos

$$
\begin{aligned}
X_{\{f, g\}}(h) & =\{\{f, g\}, h\} \\
& =-\{\{g, h\}, f\}-\{\{h, f\}, g\} \\
& =\{f,\{g, h\}\}+\{\{f, h\}, g\} \\
& =\{f,\{g, h\}\}-\{g,\{f, h\}\} \\
& =X_{f}\left(X_{g}(h)\right)-X_{g}\left(X_{f}(h)\right) \\
& =\left[X_{f}, X_{g}\right](h)
\end{aligned}
$$

Um exemplo muito importante de variedades de Poisson é $\mathfrak{g}^{*}$, vamos mostrar que o dual de uma álgebra de Lie $\mathfrak{g}$, é uma variedade de Poisson com o colchete de Poisson dado por

$$
\{f, g\}(\xi):=\left\langle\xi,\left[\mathrm{d}_{\xi} f, \mathrm{~d}_{\xi} g\right]_{\mathfrak{g}}\right\rangle, f, g \in \mathcal{C}^{\infty}\left(\mathfrak{g}^{*}\right), \xi \in \mathfrak{g}^{*} .
$$

note que $\mathrm{d}_{\xi} f$ é visto como elemento de $\mathfrak{g}$. A seguir omitiremos o índice $\mathfrak{g}$ do colchete de Lie.

Afim de mostrar que esta operação binária sobre as funções suaves em $M=\mathfrak{g}^{*}$ é de fato um colchete de Poisson é necessário verificar as quatro propriedades:

1. Anti-simetria.

$$
\begin{aligned}
\{f, g\}(\xi) & =\left\langle\xi,\left[\mathrm{d}_{\xi} f, \mathrm{~d}_{\xi} g\right]\right\rangle \\
& =-\left\langle\xi,\left[\mathrm{d}_{\xi} g, \mathrm{~d}_{\xi} f\right]\right\rangle \\
& =-\{g, f\}(\xi)
\end{aligned}
$$

2. $\mathbb{R}$-bilinearidade.

$$
\begin{aligned}
\{f, a g+b h\}(\xi) & =\left\langle\xi,\left[\mathrm{d}_{\xi} f, \mathrm{~d}_{\xi}(a g+b h)\right]\right\rangle \\
& =\left\langle\xi,\left[\mathrm{d}_{\xi} f, a \cdot \mathrm{d}_{\xi} g+b \cdot \mathrm{d}_{\xi} h\right]\right\rangle \\
& =\left\langle\xi, a\left[\mathrm{~d}_{\xi} f, \mathrm{~d}_{\xi} g\right]+b\left[\mathrm{~d}_{\xi} f, \mathrm{~d}_{\xi} h\right]\right\rangle \\
& =a\left\langle\xi,\left[\mathrm{d}_{\xi} f, \mathrm{~d}_{\xi} g\right]\right\rangle+b\left\langle\xi,\left[\mathrm{d}_{\xi} f, \mathrm{~d}_{\xi} h\right]\right\rangle \\
& =a\{f, g\}(\xi)+b\{f, h\}(\xi)
\end{aligned}
$$


3. Identidade de Jacobi.

O prezado leitor pode consultar [15], página 328 exemplo (b), onde encontrará uma identidade que ajuda na demonstração.

4. Regra de Leibniz.

$$
\begin{aligned}
\{f, g \cdot h\}(\xi) & =\left\langle\xi,\left[\mathrm{d}_{\xi} f, \mathrm{~d}_{\xi}(g \cdot h)\right]\right\rangle \\
& =\left\langle\xi,\left[\mathrm{d}_{\xi} f,\left(\mathrm{~d}_{\xi} g\right) \cdot h+g \cdot\left(\mathrm{d}_{\xi} h\right)\right]\right\rangle \\
& =\left\langle\xi,\left[\mathrm{d}_{\xi} f, \mathrm{~d}_{\xi} g\right]\right\rangle \cdot h(\xi)+g(\xi) \cdot\left\langle\xi,\left[\mathrm{d}_{\xi} f, \mathrm{~d}_{\xi} h\right]\right\rangle \\
& =\{f, g\}(\xi) \cdot h(\xi)+g(\xi) \cdot\{f, h\}(\xi) .
\end{aligned}
$$

Portanto, $\mathfrak{g}^{*}$ equipado com o colchete dado em (4.3) é uma variedade de Poisson.

Definição 4.9 Sejam $M_{1}$ e $M_{2}$ duas variedades de Poisson, definimos o produto $M_{1} \times M_{2}$ como sendo o produto usual de variedades suaves dotado com uma estrutura de Poisson $\{\cdot, \cdot\}$ tal que as aplicações projeção

$$
\operatorname{pr}_{i}: M_{1} \times M_{2} \rightarrow M_{i}
$$

são aplicações de Poisson, $e$

$$
\left\{f \circ \mathrm{pr}_{1}, g \circ \mathrm{pr}_{2}\right\}=0
$$

para toda $f \in \mathcal{C}^{\infty}\left(M_{1}\right)$ e $g \in \mathcal{C}^{\infty}\left(M_{2}\right)$.

Equivalentemente,

Definição 4.10 Sejam $\left(M_{1},\{\cdot, \cdot\}_{1}\right)$ e $\left(M_{2},\{\cdot, \cdot\}_{2}\right)$ duas variedades de Poisson. Então o produto direto $M_{1} \times M_{2}$ pode ser equipado com o seguinte colchete natural:

$$
\{f, g\}\left(x_{1}, x_{2}\right):=\left\{f\left(\cdot, x_{2}\right), g\left(\cdot, x_{2}\right)\right\}_{1}\left(x_{1}\right)+\left\{f\left(x_{1}, \cdot\right), g\left(x_{1}, \cdot\right)\right\}_{2}\left(x_{2}\right)
$$

para todo $\left(x_{1}, x_{2}\right) \in M_{1} \times M_{2}$, onde

$$
\begin{aligned}
& f_{\widehat{x}_{1}}=f\left(\cdot, x_{2}\right): x_{1} \mapsto f\left(x_{1}, x_{2}\right), f\left(\cdot, x_{2}\right) \in \mathcal{C}^{\infty}\left(M_{1}\right) \\
& f_{\widehat{x}_{2}}=f\left(x_{1}, \cdot\right): x_{2} \mapsto f\left(x_{1}, x_{2}\right), f\left(x_{1}, \cdot\right) \in \mathcal{C}^{\infty}\left(M_{2}\right)
\end{aligned}
$$

Agora podemos equipar a variedade $M=\mathfrak{g}^{*} \times \cdots \times \mathfrak{g}^{*}=\left(\mathfrak{g}^{*}\right)^{n}$ com uma estrutura de Poisson, a qual é dada por: 


$$
\{f, g\}\left(\xi_{1}, \ldots, \xi_{n}\right):=\sum_{j=1}^{n}\left\langle\xi_{j},\left[\mathrm{~d}_{\xi_{j}} f_{\widehat{\xi}_{j}}, \mathrm{~d}_{\xi_{j}} g_{\widehat{\xi_{j}}}\right]_{\mathfrak{g}}\right\rangle, \xi_{j} \in \mathfrak{g}^{*}
$$

onde

$$
f_{\widehat{\xi}_{j}}=f\left(\xi_{1}, \ldots, \widehat{\xi}_{j}, \ldots \xi_{n}\right): \xi_{j} \mapsto f\left(\xi_{1}, \ldots \xi_{n}\right), f\left(\xi_{1}, \ldots, \widehat{\xi}_{j}, \ldots \xi_{n}\right) \in \mathcal{C}^{\infty}\left(\mathfrak{g}^{*}\right)
$$

sendo $\widehat{\xi}_{j}$ representando a omissão da $j$-ésima entrada nos argumentos da função $f$. A expressão (4.4) será referida como a estrutura de Poisson produto.

Definição 4.11 Se $\left(M_{1}\{\cdot, \cdot\}_{1}\right)$ e $\left(M_{2}\{\cdot, \cdot\}_{2}\right)$ são duas variedades de Poisson, então uma aplicação suave $\varphi$ de $M_{1}$ para $M_{2}$ é chamada um morfismo de Poisson ou uma aplicação de Poisson suave se a aplicação pullback associada $\varphi^{*}: \mathcal{C}^{\infty}\left(M_{2}\right) \longrightarrow \mathcal{C}^{\infty}\left(M_{1}\right)$ é um homomorfismo de álgebra de Lie com relação aos colchetes de Poisson correspondentes.

Em outras palavras, $\varphi:\left(M_{1}\{\cdot, \cdot\}_{1}\right) \rightarrow\left(M_{2}\{\cdot, \cdot\}_{2}\right)$ é um morfismo de Poisson se

$$
\left\{\varphi^{*} f, \varphi^{*} g\right\}_{1}=\varphi^{*}\{f, g\}_{2} \text { onde } f, g \in \mathcal{C}^{\infty}\left(M_{2}\right)
$$

da Definição 2.6 de pullback de funções, podemos reescrever da seguinte forma

$$
\{f \circ \varphi, g \circ \varphi\}_{1}=\{f, g\}_{2} \circ \varphi \text { onde } f, g \in \mathcal{C}^{\infty}\left(M_{2}\right)
$$

Uma definição muito importante na geometria de Poisson é a ação de Poisson, a definição a seguir foi extraída de [22]

Definição 4.12 Considere $\Phi: G \times M \rightarrow M$ a ação do grupo de Lie $G$ em $M$. $A$ ação de $G$ é Poisson com respeito ao colchete de $M$, se para todo $g \in G$, a aplicação $\Phi_{g}$ é uma aplicação de Poisson, isto é,

$$
\left\{f \circ \Phi_{g}, h \circ \Phi_{g}\right\}=\{f, h\} \circ \Phi_{g}
$$

Proposição 4.13 Sejam $G$ e $H$ grupos de Lie e $\mathfrak{g}$ e $\mathfrak{h}$ suas álgebras de Lie, respectivamente. Seja $\phi: \mathfrak{g} \longrightarrow \mathfrak{h}$ uma aplicação linear, $\phi$ é um homomorfismo de álgebras de Lie se, e somente se, sua aplicação dual $\phi^{*}: \mathfrak{h}_{ \pm}^{*} \longrightarrow \mathfrak{g}_{ \pm}^{*}$ é uma aplicação de Poisson (linear).

A demonstração desta proposição o prezado leitor pode consultar [15], página 362. Na prova desse resultado, chega-se a seguinte igualdade:

$$
\mathrm{d}_{\xi}\left(f \circ \phi^{*}\right)=\phi \cdot \mathrm{d}_{\phi^{*}(\xi)} f, \xi \in \mathfrak{h}^{*}, f \in \mathcal{C}^{\infty}\left(\mathfrak{g}^{*}\right)
$$

usaremos esta expressão para provar que a ação coadjunta é uma ação de Poisson. Vamos aplicar essa proposição para o caso em que $G=H$. Tome 
$\phi=\operatorname{Ad}_{g^{-1}}, \phi^{*}=\operatorname{Ad}_{g}^{*}$ e $\xi \in \mathfrak{g}^{*}$, e assim a igualdade (4.5) fica da seguinte forma

$$
\mathrm{d}_{\xi}\left(f \circ \operatorname{Ad}_{g}^{*}\right)=\operatorname{Ad}_{g^{-1}} \mathrm{~d}_{\operatorname{Ad}_{g}^{*}(\xi)} f
$$

aplicando $\operatorname{Ad}_{g}$ de ambos os lados e usando o fato de que $\operatorname{Ad}_{g} \circ \operatorname{Ad}_{h}=\operatorname{Ad}_{g h}$, Proposição 2.18, obtém-se

$$
\operatorname{Ad}_{g} \mathrm{~d}_{\xi}\left(f \circ \operatorname{Ad}_{g}^{*}\right)=\mathrm{d}_{\operatorname{Ad}_{g}^{*}(\xi)} f
$$

Observação 4.14 Uma vez que $f: \mathfrak{g}^{*} \longrightarrow \mathbb{R}$ é uma função suave e $\xi \in \mathfrak{g}^{*}$, consequentemente $\operatorname{Ad}_{g}^{*} \xi \in \mathfrak{g}^{*}$, então a diferencial

$$
d_{\operatorname{Ad}_{g}^{*}(\xi)} f: T_{\operatorname{Ad}_{g}^{*}(\xi)} \mathfrak{g}^{*} \longrightarrow \mathbb{R}
$$

pode ser vista como um elemento de $\mathfrak{g}$. Daí fazer sentido $\operatorname{Ad}_{g^{-1}} d_{\operatorname{Ad}_{g}^{*}(\xi)} f$.

Afim de mostrarmos que a ação coadjunta é uma ação de Poisson, precisamos mostrar que

$$
\{f, h\}\left(\operatorname{Ad}_{g}^{*}(\xi)\right)=\left\{f \circ \operatorname{Ad}_{g}^{*}, h \circ \operatorname{Ad}_{g}^{*}\right\}(\xi)
$$

onde

$$
\{f, h\}(\xi)=\left\langle\xi,\left[\mathrm{d}_{\xi} f, \mathrm{~d}_{\xi} h\right]\right\rangle, f, h \in \mathcal{C}^{\infty}\left(\mathfrak{g}^{*}\right), \xi \in \mathfrak{g}^{*}
$$

mostremos (4.7)

$$
\begin{aligned}
\{f, h\}\left(\operatorname{Ad}_{g}^{*}(\xi)\right) & =\left\langle\operatorname{Ad}_{g}^{*}(\xi),\left[\mathrm{d}_{\operatorname{Ad}_{g}^{*}(\xi)} f, \mathrm{~d}_{\operatorname{Ad}_{g}^{*}(\xi)} h\right]\right\rangle & & \text { por (4.8) } \\
& =\left\langle\operatorname{Ad}_{g}^{*}(\xi),\left[\operatorname{Ad}_{g} \mathrm{~d}_{\xi}\left(f \circ \operatorname{Ad}_{g}^{*}\right), \operatorname{Ad}_{g} \mathrm{~d}_{\xi}\left(h \circ \operatorname{Ad}_{g}^{*}\right)\right]\right\rangle & & \text { por (4.6) } \\
& =\left\langle\operatorname{Ad}_{g}^{*}(\xi), \operatorname{Ad}_{g}\left[\mathrm{~d}_{\xi}\left(f \circ \operatorname{Ad}_{g}^{*}\right), \mathrm{d}_{\xi}\left(h \circ \operatorname{Ad}_{g}^{*}\right)\right]\right\rangle & & \text { Prop 2.18 } \\
& =\left\langle\xi,\left[\mathrm{d}_{\xi}\left(f \circ \operatorname{Ad}_{g}^{*}\right), \mathrm{d}_{\xi}\left(h \circ \operatorname{Ad}_{g}^{*}\right)\right]\right\rangle & & \text { por }(2.3) \\
& =\left\{f \circ \operatorname{Ad}_{g}^{*}, h \circ \operatorname{Ad}_{g}^{*}\right\}(\xi) & & \text { por (4.8) }
\end{aligned}
$$

portanto, a ação coadjunta é Poisson, ou seja preserva o colchete, provamos assim a seguinte proposição.

Proposição 4.15 A ação coadjunta de $G$ em $\mathfrak{g}^{*}$

$$
\begin{aligned}
\operatorname{Ad}^{*}: G \times \mathfrak{g}^{*} & \longrightarrow \mathfrak{g}^{*} \\
(g, \xi) & \longmapsto \operatorname{Ad}_{g}^{*} \xi
\end{aligned}
$$

caracterizada por

$$
\left\langle\operatorname{Ad}_{g}^{*} \xi, X\right\rangle=\left\langle\xi, \operatorname{Ad}_{g^{-1}} X\right\rangle
$$

é uma ação de Poisson, ou seja

$$
\{f, h\}\left(\operatorname{Ad}_{g}^{*}(\xi)\right)=\left\{f \circ \operatorname{Ad}_{g}^{*}, h \circ \operatorname{Ad}_{g}^{*}\right\}(\xi), f, h \in \mathcal{C}^{\infty}\left(\mathfrak{g}^{*}\right), \xi \in \mathfrak{g}^{*}, X \in \mathfrak{g}
$$


O Teorema 2.23 nos diz que para todo $\xi \in \mathfrak{g}^{*}$, a órbita coadjunta de $G$ por $\xi$, dada por

$$
\mathcal{O}_{\xi}=\left\{\operatorname{Ad}_{g}^{*} \xi \in \mathfrak{g}^{*} \mid g \in G\right\}
$$

é uma variedade simplética. Dessa forma, o dual de uma álgebra de Lie $\mathfrak{g}^{*}$ pode ser escrita como uma união de variedades simpléticas distintas e sem pontos em comum. Note que $\mathfrak{g}^{*}$ não é em si uma variedade simplética, mas sim uma variedade de Poisson.

Desde que toda variedade simplética é uma variedade de Poisson e que a variedade $\mathfrak{g}^{*}$ pode ser escrita como união disjuntas de variedades simpléticas, a saber, uma união disjunta órbitas coadjuntas, é natural querer saber se existe uma relação entre a estrutura de Poisson de $\mathfrak{g}^{*}$ e a estrutura de Poisson em cada órbita, essa pergunta é respondida na próxima proposição, a qual nos diz que existe uma relação de compatibilidade entre essas estruturas.

Teorema 4.16 O colchete Lie-Poisson e a estrutura simplética na órbita coadjunta são consistentes no seguinte sentindo: Para $f, h: \mathfrak{g}^{*} \rightarrow \mathbb{R}$ e $\mathcal{O}$ uma órbita coadjunta em $\mathfrak{g}^{*}$,

$$
\left.\{f, h\}_{+}\right|_{\mathcal{O}}=\left\{\left.f\right|_{\mathcal{O}},\left.h\right|_{\mathcal{O}}\right\}^{+}
$$

o colchete $\{f, h\}_{+}$é o colchete Lie-Poisson $(+)$, enquanto que o colchete do lado direito é o colchete de Poisson definido pela estrutura simplética $(+)$ na órbita coadjunta $\mathcal{O}$.

Para a demonstração deste teorema o prezado leitor pode consultar [15], página 461. O símbolo $(+)$ dos dois lados da igualdade dos colchetes acima serve para identificar duas estruturas de Poisson distintas que coincidem na órbita coadjunta, essas estruturas são as seguintes:

- Estrutura de Poisson em $\mathfrak{g}^{*}$

$$
\{f, h\}_{ \pm}(\xi)= \pm\left\langle\xi,\left[\mathrm{d}_{\xi} f, \mathrm{~d}_{\xi} h\right]\right\rangle
$$

- Estrutura simplética em $\mathcal{O}$ a qual define uma estrutura de Poisson

$$
\omega^{ \pm}\left(\operatorname{ad}_{X}^{*} \xi, \operatorname{ad}_{Y}^{*} \xi\right)= \pm\langle\xi,[X, Y]\rangle
$$

O parágrafo a seguir resume o conteúdo básico do teorema.

Uma maneira de produzir o colchete Lie-Poisson em $\mathfrak{g}^{*}$ é usando a restrição, como segue:

1. Para quaisquer $f, h: \mathfrak{g}^{*} \rightarrow \mathbb{R}$; 
2. Tome as restrições $\left.f\right|_{\mathcal{O}},\left.h\right|_{\mathcal{O}}$ às órbitas coadjuntas;

3. Tome o colchete de Poisson $\left\{\left.f\right|_{\mathcal{O}},\left.h\right|_{\mathcal{O}}\right\}^{+}$com respeito à estrutura simplética na órbita $\mathcal{O}$ : Para $\xi \in \mathcal{O}$ temos

$$
\left\{\left.f\right|_{\mathcal{O}},\left.h\right|_{\mathcal{O}}\right\}^{+}(\xi)=\{f, h\}_{+}(\xi)
$$

Anteriormente foi abordado que existe um único campo vetorial $X_{f}$ em uma variedade de Poisson $M$, chamado o campo vetorial Hamiltoniano de $f$, tal que para qualquer $g \in \mathcal{C}^{\infty}(M)$ temos

$$
X_{f}(g)=\{f, g\}
$$

ou seja, o colchete de Poisson permite associar um campo vetorial Hamiltoniano $X_{f}$, o qual avaliado em $\xi$, será denotado por:

$$
X_{f}(\xi)(g)=\{f, g\}(\xi)
$$

Sabemos que o colchete de Poisson em $\mathfrak{g}^{*}$ é dado por

$$
\{f, g\}(\xi)=\left\langle\xi,\left[\mathrm{d}_{\xi} f, \mathrm{~d}_{\xi} g\right]\right\rangle
$$

dessa forma de (4.10) e (4.11) temos que

$$
\begin{aligned}
X_{f}(\xi)(g) & =\left\langle\xi,\left[\mathrm{d}_{\xi} f, \mathrm{~d}_{\xi} g\right]\right\rangle \\
& =\left\langle\xi, \operatorname{ad}_{\mathrm{d}_{\xi} f} \mathrm{~d}_{\xi} g\right\rangle \\
& =-\left\langle\operatorname{ad}_{\mathrm{d}_{\xi} f}^{*} \xi, \mathrm{d}_{\xi} g\right\rangle
\end{aligned}
$$

onde usamos que $\operatorname{ad}_{X} Y=[X, Y]$ e $\left\langle\operatorname{ad}_{g} X, Y\right\rangle=-\left\langle X, \operatorname{ad}_{g}^{*} Y\right\rangle$, portanto, o campo vetorial Hamiltoniano $X_{f}$ é dado em $\xi \in \mathfrak{g}^{*}$, por

$$
X_{f}(\xi)=-\operatorname{ad}_{d_{\xi} f}^{*} \xi
$$

O teorema a seguir é um resultado fundamental em geometria de Poisson, o qual nos fornece uma informação muito importante a respeito da folheação simplética de $\mathfrak{g}^{*}$.

Teorema 4.17 As folhas simpléticas da estrutura de Poisson no dual de uma álgebra de Lie de dimensão finita arbitrária coincide com as órbitas da representação coadjunta.

Proof. Lembre que os campos vetoriais fundamentais descrevem infinitesimalmente uma ação de um grupo em uma variedade e eles geram todos os espaços tangentes às órbitas da ação, consequentemente, os campos 
vetoriais $\operatorname{ad}_{X}^{*}$ geram o espaço tangente às órbitas coadjuntas $\mathcal{O}_{\xi}$ em qualquer ponto de $\mathcal{O}_{\xi} \subset \mathfrak{g}^{*}$, de modo que para qualquer $\xi \in \mathfrak{g}^{*}$

$$
T_{\xi} \mathcal{O}_{\xi}=\left\{\operatorname{ad}_{X}^{*} \xi \mid X \in \mathfrak{g}\right\}
$$

onde $\mathcal{O}_{\xi}=\left\{\operatorname{Ad}_{g}^{*} \xi \mid g \in G\right\}$ é a órbita coadjunta passando por $\xi$. Temos, a partir de (4.12) que

$$
\operatorname{Ham}_{\xi}\left(\mathfrak{g}^{*}\right)=\left\{-\operatorname{ad}_{d_{\xi} f}^{*} \xi \mid f \in \mathcal{C}^{\infty}\left(\mathfrak{g}^{*}\right)\right\}=\left\{\operatorname{ad}_{X}^{*} \xi \mid X \in \mathfrak{g}\right\}=T_{\xi} \mathcal{O}_{\xi}
$$

onde estamos usando que, dado $X \in \mathfrak{g}$, a função linear $f$ em $\mathfrak{g}^{*}$, definida por $f_{X}(\xi)=-\langle X, \xi\rangle$, realiza $\mathrm{d}_{\xi} f=-X$. Isto prova a proposição, pois devido a regra de Leibniz, os espaços tangentes às folhas simpléticas, isto é, os espaços característicos, são gerados por campos vetoriais Hamiltonianos de funções lineares.

Observação 4.18 Note que $X_{f}(\xi)=-\operatorname{ad}_{d_{\xi} f}^{*} \xi$ é o campo vetorial Hamiltoniano gerado pela função Hamiltoniana $f_{X}(\xi)=-\langle X, \xi\rangle$. Note ainda que a aplicação momento para a ação coadjunta será dada pela inclusão natural $\mu: \mathcal{O} \subset \mathfrak{g}^{*} \rightarrow \mathfrak{g}^{*}$.

Sabemos que dadas as variedades de Poisson $\left(M_{1},\{\cdot, \cdot\}_{1}\right)$ e $\left(M_{2},\{\cdot, \cdot\}_{2}\right)$, podemos equipar a variedade produto $M_{1} \times M_{2}$ com a estrutura de Poisson produto, isto é, se $f, h: M_{1} \times M_{2} \rightarrow \mathbb{R}$, então

$$
\{f, h\}\left(x_{1}, x_{2}\right)=\left\{f_{\widehat{x}_{1}}, h_{\widehat{x}_{1}}\right\}_{1}\left(x_{1}\right)+\left\{f_{\widehat{x}_{2}}, h_{\widehat{x}_{2}}\right\}_{2}\left(x_{2}\right)
$$

onde $f_{\widehat{x}_{1}}:=f\left(\cdot, x_{2}\right): M_{1} \rightarrow \mathbb{R}$ e $f_{\widehat{x}_{2}}:=f\left(x_{1}, \cdot\right): M_{2} \rightarrow \mathbb{R}$.

Agora considere a ação coadjunta diagonal de $G$ em $M=\left(\mathfrak{g}^{*}\right)^{n}$, ou seja, $G$ age com a ação coadjunta em cada entrada de $\xi=\left(\xi_{1}, \ldots, \xi_{n}\right)$ como a seguir

$$
\begin{aligned}
\psi: G \times M & \longrightarrow M \\
(g, \xi) & \longmapsto \psi(g, \xi)=\left(\psi_{g}\left(\xi_{1}\right), \ldots, \psi_{g}\left(\xi_{n}\right)\right)
\end{aligned}
$$

onde $\psi_{g}\left(\xi_{j}\right)=\operatorname{Ad}_{g}^{*}\left(\xi_{j}\right)$ para todo $j=1, \ldots, n$. Nosso intuito é provar que esta 
ação é de fato uma ação de Poisson,

$$
\begin{aligned}
& \{f, h\}\left(\operatorname{Ad}_{g}^{*}\left(\xi_{1}, \ldots, \xi_{n}\right)\right)={ }_{(1)} \quad\{f, h\}\left(\operatorname{Ad}_{g}^{*} \xi_{1}, \ldots, \operatorname{Ad}_{g}^{*} \xi_{n}\right) \\
& ={ }_{(2)} \quad\left\{f_{\widehat{\operatorname{Ad}_{g}^{*} \xi}}, h_{\widehat{\operatorname{Ad}_{g}^{*} \xi}}\right\}\left(\operatorname{Ad}_{g}^{*}\left(\xi_{1}\right)\right)+\cdots \\
& \cdots+\left\{f_{\widehat{\operatorname{Ad}_{g}^{*} \xi}}, h_{\widehat{\operatorname{Ad}_{g}^{*} \xi_{n}}}\right\}\left(\operatorname{Ad}_{g}^{*}\left(\xi_{n}\right)\right) \\
& ={ }_{(3)}\left\langle\mathrm{Ad}_{g}^{*} \xi_{1},\left[\mathrm{~d}_{\mathrm{Ad}_{g}^{*} \xi_{1}} f_{\widehat{\operatorname{Ad}_{g}^{*} \xi_{1}}}, \mathrm{~d}_{\mathrm{Ad}_{g}^{*} \xi_{1}} h_{\widehat{\operatorname{Ad}_{g}^{*} \xi_{1}}}\right]\right\rangle+\cdots \\
& \cdots+\left\langle\operatorname{Ad}_{g}^{*} \xi_{n},\left[\mathrm{~d}_{\operatorname{Ad}_{g}^{*} \xi_{n}} f_{\widehat{\operatorname{Ad}_{g}^{*} \xi_{n}}}, \mathrm{~d}_{\operatorname{Ad}_{g}^{*} \xi_{n}} h_{\widehat{\operatorname{Ad}_{g}^{*} \xi_{n}}}\right]\right\rangle \\
& =(4) \quad\left\{f_{\widehat{\operatorname{Ad}_{g}^{*} \xi_{1}}} \circ \operatorname{Ad}_{g}^{*}, h_{\widehat{\operatorname{Ad}_{g}^{*} \xi_{1}}} \circ \operatorname{Ad}_{g}^{*}\right\}\left(\xi_{1}\right)+\cdots \\
& \cdots+\left\{f_{\widehat{\operatorname{Ad}}_{g}^{*} \xi_{n}} \circ \operatorname{Ad}_{g}^{*}, h_{\widehat{\operatorname{Ad}}_{g}^{*} \xi_{n}} \circ \operatorname{Ad}_{g}^{*}\right\}\left(\xi_{n}\right) \\
& ={ }_{(2)} \quad\left\{f \circ \mathrm{Ad}_{g}^{*}, h \circ \operatorname{Ad}_{g}^{*}\right\}\left(\xi_{1}, \ldots, \xi_{n}\right)
\end{aligned}
$$

as numerações em cada igualdade acima significam:

(1) Ação coadjunta diagonal;

(2) Definição da estrutura de Poisson produto;

(3) Estrutura de Poisson em $\mathfrak{g}^{*}$, para todo $k$ tem-se

$$
\left\{f_{\widehat{\operatorname{Ad}_{g}^{*} \xi_{k}}}, h_{\widehat{\operatorname{Ad}_{g}^{*} \xi_{k}}}\right\}\left(\operatorname{Ad}_{g}^{*}\left(\xi_{k}\right)\right)=\left\langle\operatorname{Ad}_{g}^{*} \xi_{k},\left[\mathrm{~d}_{\operatorname{Ad}_{g}^{*} \xi_{k}} f_{\widehat{\operatorname{Ad}_{g}^{*} \xi_{k}}}, \mathrm{~d}_{\operatorname{Ad}_{g}^{*} \xi_{k}} h_{\widehat{\operatorname{Ad}_{g}^{*} \xi_{k}}}\right]\right\rangle
$$

(4) A ação coadjunta é uma ação de Poisson, na demonstração da Proposição 4.15 obtemos que para todo $k$

$$
\left\{f_{\widehat{\mathrm{Ad}_{g}^{*} \xi_{k}}} \circ \mathrm{Ad}_{g}^{*}, h_{\widehat{\mathrm{Ad}_{g}^{*} \xi_{k}}} \circ \mathrm{Ad}_{g}^{*}\right\}\left(\xi_{k}\right)=\left\langle\operatorname{Ad}_{g}^{*} \xi_{k},\left[\mathrm{~d}_{\mathrm{Ad}_{g}^{*} \xi_{k}} f_{\widehat{\operatorname{Ad}}_{g}^{*} \xi_{k}}, \mathrm{~d}_{\mathrm{Ad}_{g}^{*} \xi_{k}} h_{\widehat{\mathrm{Ad}_{g}^{*} \xi_{k}}}\right]\right\rangle .
$$

Provamos assim o seguinte resultado

Proposição 4.19 A ação coadjunta diagonal

$$
\begin{aligned}
\psi: G \times\left(\mathfrak{g}^{*}\right)^{n} & \longrightarrow\left(\mathfrak{g}^{*}\right)^{n} \\
(g, \xi) & \longmapsto \psi(g, \xi)=\left(\operatorname{Ad}_{g}^{*}\left(\xi_{1}\right), \ldots, \operatorname{Ad}_{g}^{*}\left(\xi_{n}\right)\right)
\end{aligned}
$$

é uma ação de Poisson.

A aplicação momento associada à ação coadjunta é a aplicação inclusão em $\mathfrak{g}^{*}$

$$
\begin{aligned}
\mu: \mathfrak{g}^{*} & \longrightarrow \mathfrak{g}^{*} \\
\xi_{j} & \longmapsto \mu\left(\xi_{j}\right)=\xi_{j}
\end{aligned}
$$

note que esta é uma aplicação momento $G$-equivariante, pois observando o 
diagrama a seguir,

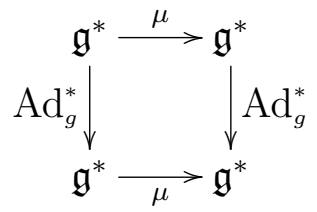

podemos perceber que

$$
\mu(g \cdot x)=\mu\left(\operatorname{Ad}_{g}^{*}(x)\right)=\operatorname{Ad}_{g}^{*}(x)=\operatorname{Ad}_{g}^{*}(\mu(x)), x \in \mathfrak{g}^{*}
$$

Sabemos ainda, que se $\mu_{1}$ é a aplicação momento associada a ação de $G$ em $M_{1}$ e $\mu_{2}$ é a aplicação momento associada a ação de $G$ em $M_{2}$ então a aplicação momento associada a ação de $G$ em $M_{1} \times M_{2}$ é dada pela soma das aplicações momento, ou seja, se $G \curvearrowright M_{1} \times M_{2}$ então a aplicação momento associada a esta ação será:

$$
\begin{aligned}
\mu: M_{1} \times M_{2} & \longrightarrow \mathfrak{g}^{*} \\
\left(x_{1}, x_{2}\right) & \longmapsto \mu\left(x_{1}, x_{2}\right)=\mu_{1}\left(x_{1}\right)+\mu_{2}\left(x_{2}\right) .
\end{aligned}
$$

Dessa forma, segue que a aplicação momento associada a ação coadjunta diagonal de $G$ em $M=\mathfrak{g}^{*} \times \cdots \times \mathfrak{g}^{*}$ será:

$$
\begin{aligned}
\mu: M=\mathfrak{g}^{*} \times \cdots \times \mathfrak{g}^{*} & \longrightarrow \mathfrak{g}^{*} \\
\xi=\left(\xi_{1}, \ldots, \xi_{n}\right) & \longmapsto \mu(\xi)=\sum_{j=1}^{n} \xi_{j},
\end{aligned}
$$

a qual é uma aplicação momento $G$-equivariante, de fato

$$
\begin{array}{rlr}
\mu(g \cdot \xi) & =\mu\left(g \cdot\left(\xi_{1}, \ldots, \xi_{n}\right)\right) & \\
& =\mu\left(\operatorname{Ad}_{g}^{*} \xi_{1}, \ldots, \operatorname{Ad}_{g}^{*} \xi_{n}\right) & \text { ação coadjunta diagonal } \\
& =\sum_{j=1}^{n} \operatorname{Ad}_{g}^{*} \xi_{j} & \\
& =\operatorname{Ad}_{g}^{*} \sum_{j=1}^{n} \xi_{j} & \\
& =\operatorname{Ad}_{g}^{*} \mu\left(\xi_{1}, \ldots, \xi_{n}\right) &
\end{array}
$$

\section{1}

\section{O colchete de Schouten}

Nosso intuito nesta seção é entender como um campo bivetorial sob certas condições define um colchete de Poisson em uma variedade de Poisson, para isto, iniciamos apresentando o colchete de Schouten, este nos diz como a partir de dois campos multivetoriais podemos obter um terceiro campo multivetorial, 
notaremos mais a frente que o colchete de Lie que estamos familiarizados é um caso particular do colchete de Schouten.

Em [16] encontramos a importante identificação abaixo, a qual será útil para o estudo do colchete de Schouten.

Proposição 4.20 Para uma variedade $M$, a atribuição

$$
\bar{\vartheta}\left(f_{1}, \ldots, f_{k}\right)=\vartheta\left(d f_{1}, \ldots, d f_{k}\right)
$$

estabelece uma correspondência um a um entre campos $k$-multivetoriais, isto é, aplicações $\mathcal{C}^{\infty}(M)$-multilineares alternadas de grau $k$ :

$$
\vartheta: \Omega^{1}(M) \times \cdots \times \Omega^{1}(M) \rightarrow \mathcal{C}^{\infty}(M)
$$

e aplicações $\mathbb{R}$ - multilineares, alternadas de grau $k$ :

$$
\bar{\vartheta}: \mathcal{C}^{\infty}(M) \times \cdots \times \mathcal{C}^{\infty}(M) \rightarrow \mathcal{C}^{\infty}(M)
$$

que são também multiderivações

$$
\bar{\vartheta}\left(f_{1}, \ldots, g h, \ldots, f_{k}\right)=g \bar{\vartheta}\left(f_{1}, \ldots, h, \ldots, f_{k}\right)+\bar{\vartheta}\left(f_{1}, \ldots, g, \ldots, f_{k}\right) h
$$

A diferencial d : $\Omega^{k}(M) \rightarrow \Omega^{k+1}(M)$ desempenha um papel crucial na geometria diferencial. A operação dual em campos multivetoriais é um tipo de colchete de Lie, que estende o colchete de Lie usual em campos vetoriais, chamado o colchete de Schouten.

Em [16] vemos que dada uma variedade de Poisson $(M,\{\cdot, \cdot\})$ podemos definir um campo bivetorial $\pi \in \mathfrak{X}^{2}(M)$ por

$$
\pi(\mathrm{d} f, \mathrm{~d} g):=\{f, g\}
$$

agora se $\pi \in \mathfrak{X}^{2}(M)$, definimos um colchete sobre as funções suaves em $M$ por

$$
\{f, g\}:=\pi(\mathrm{d} f, \mathrm{~d} g)
$$

este satisfaz as propriedades de um colchete de Poisson, exceto em geral a identidade de Jacobi, devido a isso, afim de que esse bivetor defina um colchete de Poisson em $M$, é necessário que $\pi$ satisfaça uma propriedade extra a qual está relacionada com o colchete de Schouten.

Definição 4.21 Sejam $\vartheta \in \mathfrak{X}^{k}(M)$ e $\zeta \in \mathfrak{X}^{l}(M)$ campos multivetoriais. O colchete de Schouten de $\vartheta$ e $\zeta$ é o campo multivetorial $[\vartheta, \zeta] \in \mathfrak{X}^{k+l-1}(M)$ 
definido por

$$
[\vartheta, \zeta]=\vartheta \circ \zeta-(-1)^{(k-1)(l-1)} \zeta \circ \vartheta
$$

onde estabelecemos

$$
\zeta \circ \vartheta\left(d f_{1}, \ldots, d f_{k+l-1}\right):=\sum_{\sigma}(-1)^{\sigma} \bar{\zeta}\left(\bar{\vartheta}\left(f_{\sigma(1)}, \ldots, f_{\sigma(k)}\right), f_{\sigma(k+1)}, \ldots, f_{\sigma(k+l-1)}\right)
$$

e a soma é sobre todos os $(k, l-1)$ permutações.

A fórmula (4.14) não é muito prática para contas. Observe que para um campo bivetorial $\pi \in \mathfrak{X}^{2}(M)$ com colchete associado $\{f, g\}=\pi(\mathrm{d} f, \mathrm{~d} g)$, a fórmula (4.14) fornece uma informação importante a respeito de $\pi$ afim de que defina um colchete de Poisson.

"A identidade de Jacobi para $\{\cdot, \cdot\}$ é equivalente a equação $[\pi, \pi]=0$ ".

Vamos provar isto. Sejam $f_{1}, f_{2}, f_{3} \in \mathcal{C}^{\infty}(M)$, considere as permutações a seguir

$$
\begin{aligned}
\sigma_{1}=\left(\begin{array}{ccc}
f_{1} & f_{2} & f_{3} \\
f_{1} & f_{2} & f_{3}
\end{array}\right) & \sigma_{3}=\left(\begin{array}{ccc}
f_{1} & f_{2} & f_{3} \\
f_{2} & f_{1} & f_{3}
\end{array}\right) & \sigma_{5}=\left(\begin{array}{lll}
f_{1} & f_{2} & f_{3} \\
f_{3} & f_{1} & f_{2}
\end{array}\right) \\
\sigma_{2}=\left(\begin{array}{lll}
f_{1} & f_{2} & f_{3} \\
f_{1} & f_{3} & f_{2}
\end{array}\right) & \sigma_{4}=\left(\begin{array}{lll}
f_{1} & f_{2} & f_{3} \\
f_{2} & f_{3} & f_{1}
\end{array}\right) & \sigma_{6}=\left(\begin{array}{lll}
f_{1} & f_{2} & f_{3} \\
f_{3} & f_{2} & f_{1}
\end{array}\right)
\end{aligned}
$$

inicialmente determinamos os sinais destas permutações

$$
\begin{array}{ll}
\operatorname{sign}\left(\sigma_{1}\right)=(-1)^{0}=1 & \operatorname{sign}\left(\sigma_{4}\right)=(-1)^{2}=1 \\
\operatorname{sign}\left(\sigma_{2}\right)=(-1)^{1}=-1 & \operatorname{sign}\left(\sigma_{5}\right)=(-1)^{2}=1 \\
\operatorname{sign}\left(\sigma_{3}\right)=(-1)^{1}=-1 & \operatorname{sign}\left(\sigma_{6}\right)=(-1)^{3}=-1
\end{array}
$$

seja $\pi$ um campo bivetorial, $\pi \in \mathfrak{X}^{2}(M)$, do colchete de Schouten temos

$$
[\pi, \pi]=\pi \circ \pi-(-1)^{(2-1)(2-1)} \pi \circ \pi \Rightarrow[\pi, \pi]=2 \pi \circ \pi
$$

temos que $k=l=2 \Rightarrow k+l-1=3$, dessa forma da Definição 4.21 temos

$$
\pi \circ \pi\left(\mathrm{d} f_{1}, \mathrm{~d} f_{2}, \mathrm{~d} f_{3}\right)=\sum_{\sigma}(-1)^{\sigma} \bar{\pi}\left(\bar{\pi}\left(f_{\sigma(1)}, f_{\sigma(2)}\right), f_{\sigma(3)}\right)
$$

agora, fazendo a identificação dada na Proposição 4.20

$$
\bar{\pi}\left(f_{1}, \ldots, f_{k}\right)=\pi\left(\mathrm{d} f_{1}, \ldots, \mathrm{d} f_{k}\right)
$$


e o colchete associado $\{f, g\}=\pi(\mathrm{d} f, \mathrm{~d} g)$, assim (4.16) fica da seguinte forma,

$$
\begin{aligned}
\pi \circ \pi\left(\mathrm{d} f_{1}, \mathrm{~d} f_{2}, \mathrm{~d} f_{3}\right)= & \bar{\pi}\left(\bar{\pi}\left(f_{1}, f_{2}\right), f_{3}\right)-\bar{\pi}\left(\bar{\pi}\left(f_{1}, f_{3}\right), f_{2}\right)-\bar{\pi}\left(\bar{\pi}\left(f_{2}, f_{1}\right), f_{3}\right) \\
& +\bar{\pi}\left(\bar{\pi}\left(f_{2}, f_{3}\right), f_{1}\right)+\bar{\pi}\left(\bar{\pi}\left(f_{3}, f_{1}\right), f_{2}\right)-\bar{\pi}\left(\bar{\pi}\left(f_{3}, f_{2}\right), f_{1}\right) \\
= & \bar{\pi}\left(\bar{\pi}\left(f_{1}, f_{2}\right), f_{3}\right)+\bar{\pi}\left(\bar{\pi}\left(f_{3}, f_{1}\right), f_{2}\right)+\bar{\pi}\left(\bar{\pi}\left(f_{1}, f_{2}\right), f_{3}\right) \\
& +\bar{\pi}\left(\bar{\pi}\left(f_{2}, f_{3}\right), f_{1}\right)+\bar{\pi}\left(\bar{\pi}\left(f_{3}, f_{1}\right), f_{2}\right)+\bar{\pi}\left(\bar{\pi}\left(f_{2}, f_{3}\right), f_{1}\right) \\
= & 2\left[\bar{\pi}\left(\bar{\pi}\left(f_{1}, f_{2}\right), f_{3}\right)+\bar{\pi}\left(\bar{\pi}\left(f_{2}, f_{3}\right), f_{1}\right)+\bar{\pi}\left(\bar{\pi}\left(f_{3}, f_{1}\right), f_{2}\right)\right] \\
= & 2\left[\pi\left(\pi\left(\mathrm{d} f_{1}, \mathrm{~d} f_{2}\right), \mathrm{d} f_{3}\right)+\pi\left(\pi\left(\mathrm{d} f_{2}, \mathrm{~d} f_{3}\right), \mathrm{d} f_{1}\right)+\right. \\
& \left.\pi\left(\pi\left(\mathrm{d} f_{3}, \mathrm{~d} f_{1}\right), \mathrm{d} f_{2}\right)\right] \\
= & 2\left[\left\{\left\{f_{1}, f_{2}\right\}, f_{3}\right\}+\left\{\left\{f_{2}, f_{3}\right\}, f_{1}\right\}+\left\{\left\{f_{3}, f_{1}\right\}, f_{2}\right\}\right]
\end{aligned}
$$

desta última e de (4.15) temos que

$$
\frac{1}{4}[\pi, \pi]\left(\mathrm{d} f_{1}, \mathrm{~d} f_{2}, \mathrm{~d} f_{3}\right)=\left\{\left\{f_{1}, f_{2}\right\}, f_{3}\right\}+\left\{\left\{f_{3}, f_{1}\right\}, f_{2}\right\}+\left\{\left\{f_{2}, f_{3}\right\}, f_{1}\right\}
$$

assim, a identidade de Jacobi para $\{\cdot, \cdot\}$ é equivalente a equação $[\pi, \pi]=0$ e concluímos assim a seguinte proposição.

Proposição 4.22 Seja $(M,\{\cdot, \cdot\})$ uma variedade de Poisson. Então o campo bivetorial $\pi \in \mathfrak{X}^{2}(M)$ satisfaz

$$
[\pi, \pi]=0
$$

reciprocamente, todo campo bivetorial $\pi \in \mathfrak{X}^{2}(M)$ satisfazendo esta relação define um colchete de Poisson por $\{f, g\}=\pi(d f, d g)$.

Consequentemente podemos substituir nossa inicial Definição 4.1 de um colchete de Poisson, por:

Definição 4.23 Um campo bivetorial $\pi \in \mathfrak{X}^{2}(M)$ satisfazendo $[\pi, \pi]=0$ é chamado uma estrutura de Poisson em $M$. Um par $(M, \pi)$, onde $\pi$ é uma estrutura de Poisson em $M$, é chamado uma variedade de Poisson.

Usaremos neste momento a seguinte notação

$$
\pi[f \wedge g]=\pi(\mathrm{d} f, \mathrm{~d} g)=\{f, g\}=X_{f}(g) .
$$

A derivada de Lie de um campo $k$-vetorial arbitrário $Y$ com respeito ao campo vetorial $X$, é o campo $k$-vetorial $\mathcal{L}_{X} Y$, definido por

$$
\mathcal{L}_{X} Y\left[f_{1} \wedge \cdots \wedge f_{k}\right]:=X\left(Y\left[f_{1} \wedge \cdots \wedge f_{k}\right]\right)-\sum_{i=1}^{k} Y\left[f_{1} \wedge \cdots \wedge X\left(f_{i}\right) \wedge f_{k}\right]
$$

onde $f_{1}, \ldots, f_{k} \in \mathcal{C}^{\infty}(M)$. A derivada de Lie $\mathcal{L}_{X} Y$ de um campo $k$-vetorial $Y$ em $M$ mede como $Y$ varia na direção de $X$, consequentemente $\mathcal{L}_{X} Y=0$ se, e somente se, $Y$ é constante nas curvas integrais do campo vetorial $X$. 
A identidade de Jacobi tem uma interessante consequência dada pela seguinte proposição

Proposição $4.24 \operatorname{Sejam}(M,\{\cdot, \cdot\})$ uma variedade de Poisson e $h \in \mathcal{C}^{\infty}(M)$. O campo vetorial Hamiltoniano $X_{h}$ deixa $\{\cdot, \cdot\}$ invariante.

Proof. Seja $\pi$ o campo bivetorial que corresponde a $\{\cdot, \cdot\}$, de modo que $\pi[f \wedge g]=\{f, g\}$ para $f, g \in \mathcal{C}^{\infty}(M)$. Note que para $X_{h}$ deixar $\{\cdot, \cdot\}$ invariante, significa que $\pi$ é constante nas curvas integrais do campo $X_{h}$, portanto precisamos mostrar que $\mathcal{L}_{X_{h}} \pi=0$. Sejam $f, g \in \mathcal{C}^{\infty}(M)$, usando (4.18) obtemos que

$$
\begin{array}{rlr}
\mathcal{L}_{X_{h}} \pi[f \wedge g] & =X_{h}(\pi[f \wedge g])-\pi\left[X_{h}(f) \wedge g\right]-\pi\left[f \wedge X_{h}(g)\right] & \operatorname{de}(4.18) \\
& =X_{h}\{f, g\}-\left\{X_{h}(f), g\right\}-\left\{f, X_{h}(g)\right\} & \operatorname{de}(4.17) \\
& =\{h,\{f, g\}\}-\{\{h, f\}, g\}-\{f,\{h, g\}\} & \\
& =\{h,\{f, g\}\}+\{g,\{h, f\}\}+\{f,\{g, h\}\} &
\end{array}
$$

a qual é igual a zero devido a identidade de Jacobi, portanto campos vetoriais Hamiltonianos deixam o colchete invariante.

Exemplo 4.25 Verifiquemos que $\mathcal{L}_{X} Y=[X, Y]$ para quaisquer $X, Y \in$ $\mathfrak{X}(M)$.

De fato, se $f \in \mathcal{C}^{\infty}(M)$ então (4.18) implica que

$$
\begin{aligned}
\left(\mathcal{L}_{X} Y\right)(f) & =X(Y(f))-Y(X(f)) \\
& =(X Y-Y X)(f) \\
& =[X, Y](f)
\end{aligned}
$$

Definição 4.26 Seja $(M,\{\cdot, \cdot\})$ uma variedade de Poisson e seja $X \in \mathfrak{X}(M)$. Então $X$ é chamado um campo vetorial de Poisson se $X$ deixa $\{\cdot, \cdot\}$ invariante, isto é, $\mathcal{L}_{X} \pi=0$, onde $\pi$ denota o campo bivetorial que corresponde $a\{\cdot, \cdot\}$.

Esta definição nos diz que todo campo vetorial Hamiltoniano é um campo vetorial de Poisson, no entanto a recíproca não precisa ser verdade. 


\section{2 \\ Quocientes}

Nesta seção enunciamos o que é um $G$-espaço de Poisson e um $G$-espaço Hamiltoniano, apresentamos alguns resultados clássicos que utilizaremos mais adiante, como é o caso da estrutura de Poisson no espaço de órbitas $M / G$, claro que sob condições adequadas da ação e da variedade, abordamos um pouco a respeito do mapa momento e de sua $G$-equivariância e por fim enunciamos o resultado que nos informa que as componentes conexas de $M / / G$ são folhas simpléticas do espaço $\left(M / G, \pi_{M / G}\right)$.

Definição 4.27 Uma ação de Poisson de um grupo de Lie G em uma variedade de Poisson $(M, \pi)$ é uma ação suave tal que para cada $g \in G$ a translação $\psi_{g}: M \rightarrow M$ é um difeomorfismo de Poisson. Chamamos a tripla $(M, \pi, G)$ um G-espaço de Poisson.

Portanto, para uma ação de Poisson, as translações $\psi_{g}: M \rightarrow M$ aplicam folhas simpléticas de $M$ em folhas simpléticas.

Teorema 4.28 Seja $(M, \pi, G)$ um $G$-espaço de Poisson livre e próprio. Então existe uma única estrutura de Poisson quociente $\pi_{M / G}$ em $M / G$ para a qual a aplicação $q: M \rightarrow M / G$ é Poisson. Se $H \in \mathcal{C}^{\infty}(M)$ é uma função $G$-invariante então o campo vetorial Hamiltoniano $X_{H} \in \mathfrak{X}(M)$ projeta-se para um campos vetorial Hamiltoniano $X_{h} \in \mathfrak{X}(M / G)$.

Proof. Provaremos apenas a primeira parte deste teorema. Assumimos primeiro que $M / G$ é Poisson e provamos a unicidade. Seja

$$
q: M \rightarrow M / G
$$

a aplicação quociente. A condição de $q$ ser Poisson significa que para funções $f, k: M / G \rightarrow \mathbb{R}$, temos

$$
\{f, k\} \circ q=\{f \circ q, k \circ q\}
$$

onde os colchetes acima são em $M / G$ e $M$, respectivamente.

A função $\bar{f}=f \circ q$ é a única função $G$-invariante que projeta-se a $f$, em outras palavras, se $[z] \in M / G$ é uma classe de equivalência, através da qual $g_{1} \cdot z$ e $g_{2} \cdot z$ são equivalentes, fazemos

$$
\bar{f}(g \cdot z)=f([z]) \forall g \in G .
$$

Obviamente, isto define $\bar{f}$ univocamente de modo a

$$
\bar{f}=f \circ q .
$$


Podemos também caracterizar isto dizendo que $\bar{f}$ assume o valor $f([z])$ em toda a órbita $G \cdot z$, podemos escrever (4.19) como

$$
\{f, k\} \circ q=\{\bar{f}, \bar{k}\}
$$

Desde que $q$ é sobrejetiva, isto determina $\{f, k\}$ unicamente.

Podemos também usar (4.19) para definir $\{f, k\}$. Primeiro note que

$$
\begin{aligned}
\{\bar{f}, \bar{k}\}(g \cdot z) & =\left(\{\bar{f}, \bar{k}\} \circ \Phi_{g}\right)(z) \\
& =\left\{\bar{f} \circ \Phi_{g}, \bar{k} \circ \Phi_{g}\right\}(z) \\
& =\{\bar{f}, \bar{k}\}(z)
\end{aligned}
$$

desde que $\Phi_{g}$ é Poisson e desde que $\bar{f}$ e $\bar{k}$ são constantes nas órbitas. Assim, $\{\bar{f}, \bar{k}\}$ é também constante na órbita e assim define $\{f, k\}$ unicamente.

Resta mostrar que $\{f, k\}$ assim definido satisfaz as propriedades de uma estrutura de Poisson. No entanto, todas as propriedades seguem imediatamente do que ocorre em $M$. Por exemplo, se escrevemos a identidade de Jacobi em $M$,

$$
0=\{\{\bar{f}, \bar{k}\}, \bar{l}\}+\{\{\bar{k}, \bar{l}\}, \bar{f}\}+\{\{\bar{l}, \bar{f}\}, \bar{k}\}
$$

isto dá, por construção

$$
\begin{aligned}
0 & =\{\{f, k\} \circ q, l \circ q\}+\{\{k, l\} \circ q, f \circ q\}+\{\{l, f\} \circ q, k \circ q\} \\
& =\{\{f, k\}, l\} \circ q+\{\{k, l\}, f\} \circ q+\{\{l, f\}, k\} \circ q
\end{aligned}
$$

pela sobrejetividade de $q$, a identidade de Jacobi é válida em $M / G$

Observação 4.29 Fornecer uma aplicação linear $\hat{\mu}: \mathfrak{g} \rightarrow \mathcal{C}^{\infty}(M), \xi \mapsto \mu_{\xi}$, é algo como dar uma aplicação suave $\mu: M \rightarrow \mathfrak{g}^{*}:$ um determina o outro através da relação

$$
\mu_{\xi}(x)=\langle\mu(x), \xi\rangle
$$

Definição 4.30 Uma aplicação momento para uma ação de um grupo de Lie $G$ em uma variedade de Poisson $(M, \pi)$ é uma aplicação suave $\mu: M \rightarrow \mathfrak{g}^{*}$ tal que a condição de aplicação momento vale:

$$
X_{\xi}=-\pi^{\sharp}\left(d \mu_{\xi}\right), \forall \xi \in \mathfrak{g}
$$

Dada uma aplicação momento $\mu: M \rightarrow \mathfrak{g}^{*}$ para uma ação $G \times M \rightarrow M$ em uma variedade de Poisson, diremos que $\mu$ é uma aplicação momento $G$-equivariante se:

$$
\mu(g \cdot x)=\operatorname{Ad}_{g}^{*} \mu(x), \forall x \in M, g \in G
$$


para todo $g \in G$, isto é, o diagrama a seguir comuta

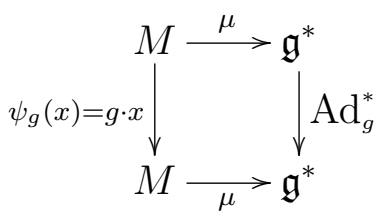

Definição 4.31 Uma ação de Poisson $G \times M \rightarrow M$ em uma variedade de Poisson $(M, \pi)$ é chamada uma ação Hamiltoniana se admite uma aplicação momento $G$-equivariante $\mu: M \rightarrow \mathfrak{g}^{*}$. A quádrupla $(M, \pi, G, \mu)$ é chamada um G-espaço Hamiltoniano.

Em [16] encontramos os seguintes comentários para um $G$-espaço Hamiltoniano $(M, \pi, G, \mu)$ :

1. A aplicação momento $\mu: M \rightarrow \mathfrak{g}^{*}$ é uma aplicação de Poisson;

2. A aplicação $\mathfrak{g} \rightarrow \mathcal{C}^{\infty}, \xi \mapsto \mu_{\xi}$ é um homomorfismo de álgebra de Lie.

A seguir temos o principal resultado de $G$-espaços Hamiltonianos o qual estabelece uma relação muito importante entre os quocientes $M / / G$ e $M / G$.

Teorema 4.32 (Meyer-Marsden-Weinstein) Seja $\quad(M, \pi, G, \mu) \quad$ um $G$-espaço hamiltoniano livre e próprio. Então $0 \in \mathfrak{g}^{*}$ é um valor regular de $\mu e$

$$
M / / G:=\mu^{-1}(0) / G
$$

é uma subvariedade de Poisson de $\left(M / G, \pi_{M / G}\right)$. Se $\pi_{M}$ é simplético, então $\pi_{M / / G}$ é também simplético, e as componentes conexas de $M / / G$ são folhas simpléticas de $\left(M / G, \pi_{M / G}\right)$.

Observação 4.33 Temos que 0 é um valor regular de $\mu$ e $\mu^{-1}(0)$ é uma subvariedade fechada de $M$. Sejam $f$ e $k$ duas funções quaisquer em $\mu^{-1}(0) / G$ visto como funções $G$-invariantes em $\mu^{-1}(0)$. Estendendo-as para duas funções $G$-invariantes $\bar{f}$ e $\bar{k}$ em uma vizinhança de $\mu^{-1}(0)$ em $M$. Desde que a estrutura de Poisson em $M$ é $G$-invariante, o colchete de Poisson $\{\bar{f}, \bar{k}\}$ é também $G$-invariante. Note ainda que a restrição de $\{\bar{f}, \bar{k}\} a \mu^{-1}(0)$ depende apenas de $f$ e $k$ mas não das extensões $\bar{f}$ e $\bar{k}$. Podemos definir o colchete de Poisson de $f, k$ em $\mu^{-1}(0) / G$ ser a projeção de $\left.\{\bar{f}, \bar{k}\}\right|_{\mu^{-1}(0)} e m \mu^{-1}(0) / G$

Quando $(M, \pi, G, \mu)$ é $G$-espaço Hamiltoniano simplético livre e próprio, chama-se $M / / G$ o quociente simplético de $(M, \pi, G, \mu)$. Na configuração mais geral, onde $\pi_{M}$ pode ser degenerado, chamaremos $M / / G$ quociente Hamiltoniano de $(M, \pi, G, \mu)$. 


\section{5}

\section{A ação coadjunta de $S U(2)$ em $\mathfrak{s u}^{*}(2)$}

Nesta parte daremos ênfase ao grupo de Lie compacto e semisimples ${ }^{1}$ $S U(2)$, mostraremos que a órbita coadjunta pode ser vista como uma esfera em $\mathbb{R}^{3}$, obtemos a forma explícita da 2-formas simplética na órbita e em seguida faremos o estudo da ação coadjunta diagonal de $S U(2)$ na variedade de Poisson obtida pelo produto de $\mathfrak{s u}^{*}(2)$.

A variedade $\mathfrak{g}^{*}$ carrega uma estrutura de Poisson natural a qual é invariante com respeito a ação coadjunta de $G$ em $\mathfrak{g}^{*}$, e esta estrutura é dada por

$$
\{f, g\}(\xi):=\left\langle\xi,\left[\mathrm{d}_{\xi} f, \mathrm{~d}_{\xi} g\right]_{\mathfrak{g}}\right\rangle, f, g \in \mathcal{C}^{\infty}\left(\mathfrak{g}^{*}\right) \text { e } \xi \in \mathfrak{g}^{*} .
$$

Vale ressaltar, que em geral, $\mathfrak{g}^{*}$ com esse colchete de Poisson não é uma variedade simplética, por exemplo, no caso $\mathfrak{g}=\mathfrak{s u}(2)$, o espaço $\mathfrak{g}^{*}$ é tridimensional, logo não pode ser uma variedade simplética.

Geralmente não é fácil descrever as folhas simpléticas de uma variedade de Poisson, no entanto, para o caso do dual de uma álgebra de Lie, a descrição dessas folhas é mais simples, pois as folhas simpléticas coincidem com as órbitas da ação coadjunta de $G$ em $\mathfrak{g}^{*}$, resultado este que foi provado no Teorema 4.17.

Sabemos que $S U(2)$ é um grupo de Lie compacto e semisimples, pois sua álgebra de Lie é semisimples, e como a álgebra de Lie de $S U(2)$, de acordo com a Proposição 6.22, é dada por

$$
\mathfrak{s u}(2)=\left\{X \in M_{2}(\mathbb{C}) \mid X+X^{*}=0_{2} \text { e } \operatorname{tr} X=0\right\}
$$

fixando uma matriz $X \in \mathfrak{s u}(2)$, temos que $X$ é anti-Hermitiana, logo os autovalores são zero ou imaginário puro. Uma vez que para calcular os autovalores de $X$ precisamos determinar as raízes do polinômio característico

$$
p(x)=\operatorname{det}(X-x I)
$$

onde

$$
X=\left(\begin{array}{cc}
i a & b+i c \\
-b+i c & -i a
\end{array}\right), a, b, c \in \mathbb{R} \text { e } I=\left(\begin{array}{ll}
1 & 0 \\
0 & 1
\end{array}\right)
$$

${ }^{1}$ A definição de grupo de Lie semisimples é abordada na seção 8.1 
fazendo os cálculos obtemos que

$$
p(x)=0 \Leftrightarrow a^{2}+b^{2}+c^{2}+x^{2}=0
$$

dessa forma $x= \pm i \lambda, \quad \lambda \in \mathbb{R}$. Como cada matriz anti-Hermitiana pode ser diagonalizada, com a diagonal sendo formada pelos autovalores, conjugando por uma matriz unitária especial, dessa forma, existe $g \in S U(2)$ tal que

$$
g \cdot X \cdot g^{-1}=\left(\begin{array}{cc}
i \lambda & 0 \\
0 & -i \lambda
\end{array}\right)
$$

mas isto significa que existe $H \in \mathfrak{s u}(2)$ tal que

$$
H:=\operatorname{Ad}_{g} X=g \cdot X \cdot g^{-1}=i\left(\begin{array}{cc}
\lambda & 0 \\
0 & -\lambda
\end{array}\right)
$$

assim, $H$ tem duas possibilidades, $H=0$, matriz nula de ordem 2, ou $H=\operatorname{diag}(i \lambda,-i \lambda)$. Vale ressaltar que matrizes da forma

$$
X=\left(\begin{array}{cc}
i a & b+i c \\
-b+i c & -i a
\end{array}\right), a, b, c \in \mathbb{R}
$$

tem a mesma diagonalização se, e somente se, elas tem o mesmo determinante, a saber $d=a^{2}+b^{2}+c^{2}$.

Em (5.1), $H=0$ ocorre quando o autovalor é nulo, $\lambda=0$, a órbita será um ponto, apenas a origem, e isto acontece quando $X$ é a matriz nula, agora se $\lambda \neq 0, \log$ $H$ é diferente da matriz nula, a órbita será a variedade bandeira complexa ${ }^{2} \mathbf{F}_{\mathbb{C}}(1,1) \simeq \mathbb{C P}^{1}$ que é difeomórfica a 2 -esfera $\mathbb{S}_{|\lambda|}^{2}$.

Mostraremos que de fato a órbita adjunta de $S U(2)$ por $X$ é a 2-esfera $\mathbb{S}_{|\lambda|}^{2}$ com raio $|\lambda|$, onde $\lambda$ é o autovalor do ponto $X \in \mathfrak{s u}(2)$. A equação (5.1) nos fornece a informação de que em qualquer órbita existe um ponto $H$ da forma diagonal. Faremos uso da bijeção entre $\mathfrak{s u}(2)$ e $\mathbb{R}^{3}$, se $Y \in \mathfrak{s u}(2)$, então existem números reis $m, k, l \in \mathbb{R}$ tais que

$$
\mathfrak{s u}(2) \ni Y=\left(\begin{array}{cc}
i m & k+i l \\
-k+i l & -i m
\end{array}\right) \simeq(m, k, l)=v \in \mathbb{R}^{3}
$$

nosso intuito é provar que para qualquer $g \in S U(2)$, o vetor $v_{g}$ em $\mathbb{R}^{3}$ correspondente a $\operatorname{Ad}_{g} Y$ pela bijeção acima, possui a mesma norma de $v$, ou seja, $\left\|v_{g}\right\|$ é constante independente da variação de $g$. Seja $g \in S U(2)$, sabemos que $g$ pode ser escrito da forma

\footnotetext{
${ }^{2}$ Este resultado é provado na Proposição 6.24
} 


$$
g=\left(\begin{array}{cc}
a & -\bar{b} \\
b & \bar{a}
\end{array}\right), a, b \in \mathbb{C} \text { e }|a|^{2}+|b|^{2}=1
$$

como em toda órbita existe um ponto $H$ da forma $H=i \operatorname{diag}(\lambda,-\lambda)$, é suficiente fazer a prova usando $H$, dessa forma

$$
g \cdot H \cdot g^{-1}=i \lambda\left(\begin{array}{cc}
|a|^{2}-|b|^{2} & 2 a \bar{b} \\
2 \bar{a} b & |b|^{2}-|a|^{2}
\end{array}\right)
$$

escrevendo $a=x+i y$ e $b=r+i t$, onde $a, b, r, t \in \mathbb{R}$, obtemos

$$
g \cdot H \cdot g^{-1}=\left(\begin{array}{cc}
i \lambda\left(x^{2}+y^{2}-r^{2}-t^{2}\right) & 2 \lambda(x t-y r+i(x r+y t)) \\
2 \lambda(y r-x t+i(x r+y t)) & i \lambda\left(r^{2}+t^{2}-x^{2}-y^{2}\right)
\end{array}\right)
$$

pela bijeção com $\mathbb{R}^{3}$ obtemos o vetor

$$
v_{g}=\left(\lambda\left(x^{2}+y^{2}-r^{2}-t^{2}\right), 2 \lambda(x t-y r), 2 \lambda(x r+y t)\right)
$$

resta-nos verificar se $\left\|v_{g}\right\|$ é constante, para isto analisemos

$$
\begin{aligned}
\left\|v_{g}\right\|^{2} & =\left(\lambda\left(x^{2}+y^{2}-r^{2}-t^{2}\right)\right)^{2}+(2 \lambda(x t-y r))^{2}+(2 \lambda(x r+y t))^{2} \\
& =\lambda^{2}\left(x^{4}+y^{4}+r^{4}+t^{4}+2\left(x^{2} y^{2}+r^{2} t^{2}+x^{2} r^{2}+x^{2} t^{2}+y^{2} r^{2}+y^{2} t^{2}\right)\right) \\
& =\lambda^{2}\left(x^{2}+y^{2}+r^{2}+t^{2}\right)^{2} \\
& =\lambda^{2}\left(|a|^{2}+|b|^{2}\right)^{2} \\
& =\lambda^{2}
\end{aligned}
$$

assim, $\left\|v_{g}\right\|=|\lambda|$, note que tínhamos $H=\operatorname{diag}(i \lambda,-i \lambda)$, com vetor correspondente em $\mathbb{R}^{3}$,

$$
v_{H}=(\lambda, 0,0) \Rightarrow\left\|v_{H}\right\|^{2}=\lambda^{2} \Rightarrow\left\|v_{H}\right\|=|\lambda|
$$

como $g$ é qualquer em $S U(2)$, segue que $\left\|v_{g}\right\|=|\lambda|$ para todo $g \in S U(2)$, assim a órbita adjunta de $S U(2)$ por $H$ é a esfera de raio $|\lambda|$ em $\mathbb{R}^{3}$, onde $\lambda$ é o autovalor de $H$ e consequentemente da matriz $X \in \mathfrak{s u}(2)$ tomada inicialmente, portanto

$$
\mathcal{O}_{H}=\mathbb{S}_{|\lambda|}^{2}
$$

dessa forma $\mathcal{O}_{H}=\mathcal{O}_{X}$, pois pelo Teorema 2.12 duas órbitas são iguais ou disjuntas.

Uma vez que $S U(2)$ é um grupo de Lie semisimples, segue da Observação 8.19 que as órbitas adjunta e coadjunta são equivalentes, concluímos dessa forma que as órbitas coadjuntas de $S U(2)$ são esferas de dimensão 2 ou um 
ponto, neste último caso a origem.

Além disso, como ações de grupos de Lie compactos são sempre próprias, assim $S U(2) \curvearrowright \mathfrak{s u}^{*}(2)$, pela ação coadjunta é uma ação própria.

Sabemos do Teorema 2.23 que as órbitas coadjuntas carregam uma estrutura simplética natural que é conhecida como a forma de Kirillov-Kostant-Souriau, a qual é dada por:

$$
\omega_{\xi}\left(\operatorname{ad}_{u}^{*}(\xi), \operatorname{ad}_{v}^{*}(\xi)\right)=\langle\xi,[u, v]\rangle, u, v \in \mathfrak{g}, \xi \in \mathfrak{g}^{*}
$$

lembrando que

$$
\operatorname{ad}_{u} v=\left.\frac{d}{d t}\right|_{t=0} \operatorname{Ad}_{\exp (t u)} v=[u, v] \mathrm{e}\left\langle\operatorname{ad}_{u}^{*} \xi, v\right\rangle=-\left\langle\xi, \operatorname{ad}_{u} v\right\rangle
$$

e que o espaço tangente a $\mathcal{O}_{H}$ no ponto $H$ é dado por

$$
T_{H} \mathcal{O}_{H}=\left\{\operatorname{ad}_{u}^{*} H \mid u \in \mathfrak{g}\right\}
$$

Vamos determinar essa forma simplética, para isto, sejam $H$ como em (5.1) e $u, v \in \mathfrak{s u}(2), \log$ existem $a, b, c, f, g, h \in \mathbb{R}$ tais que

$$
u=\left(\begin{array}{cc}
i a & b+i c \\
-b+i c & -i a
\end{array}\right), v=\left(\begin{array}{cc}
i f & g+i h \\
-g+i h & -i f
\end{array}\right)
$$

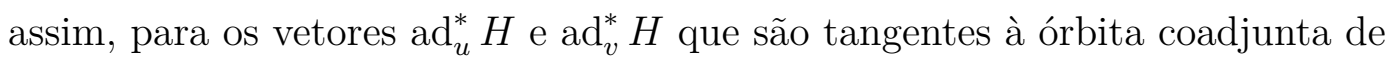
$S U(2)$ por $H$ no ponto $H$, temos

$$
\omega_{H}\left(\operatorname{ad}_{u}^{*} H, \operatorname{ad}_{v}^{*} H\right)=\langle H,[u, v]\rangle=\frac{1}{2} \operatorname{tr}\left(H \cdot[u, v]^{*}\right)
$$

para obtermos a 2-forma, calculamos

$$
[u, v]=\left(\begin{array}{cc}
2 i(b h-c g) & 2(c f-a h)+2 i(a g-b f) \\
2(a h-c f)+2 i(a g-b f) & 2 i(c g-b h)
\end{array}\right)
$$

e o produto

$$
H \cdot[u, v]^{*}=\left(\begin{array}{cc}
2 \lambda(b h-c g) & \cdots \\
\cdots & 2 \lambda(b h-c g)
\end{array}\right)
$$

e assim,

$$
\frac{1}{2} \operatorname{tr}\left(H \cdot[u, v]^{*}\right)=2 \lambda(b h-c g)
$$

para os complexos $z=b+i c$ e $w=g+i h$ temos

$$
z \bar{w}-w \bar{z}=2 i(c g-h b) \Rightarrow i \lambda(z \bar{w}-w \bar{z})=\frac{1}{2} \operatorname{tr}\left(H \cdot[u, v]^{*}\right)
$$


portanto

$$
\omega_{H}\left(\operatorname{ad}_{u}^{*} H, \operatorname{ad}_{v}^{*} H\right)=i \lambda(z \bar{w}-w \bar{z}) .
$$

Do Teorema 4.17 temos que $\left(\mathbb{S}_{|\lambda|}^{2}, \omega_{H}\right)$ é folha simplética de $\mathfrak{s u}^{*}(2)$, ainda mais, as folhas simpléticas são as esferas e a origem.

Dada a ação coadjunta

$$
\begin{aligned}
\operatorname{Ad}^{*}: S U(2) \times \mathfrak{s u}^{*}(2) & \longrightarrow \mathfrak{s u}^{*}(2) \\
(g, \xi) & \longmapsto \operatorname{Ad}_{g}^{*} \xi
\end{aligned}
$$

escolhendo $\xi \in \mathfrak{s u}^{*}(2)$ tal que os autovalores não sejam nulos, dessa forma, pelo exposto anteriormente, a órbita $\mathcal{O}_{\xi} \simeq \mathbb{S}_{|\lambda|}^{2}$, tomando a restrição da ação coadjunta $\left.\mathrm{Ad}^{*}\right|_{S U(2) \times \mathcal{O}_{\xi}}$, pelo Teorema 2.23 temos que esta é Hamiltoniana com aplicação momento dada pela inclusão

$$
\begin{aligned}
\mu: \mathcal{O}_{\xi} & \longrightarrow \mathfrak{s u}^{*}(2) \\
\xi & \longmapsto \mu(\xi)=\xi
\end{aligned}
$$

Note que a ação de $S U(2)$ é própria pois o grupo $S U(2)$ é compacto, iremos mostrar que a ação coadjunta $S U(2)$ não é uma ação livre. Pelo o que vimos anteriormente, concluímos que se $\mathcal{O}_{\xi}$ é a órbita coadjunta de $S U(2)$ por $\xi \in \mathfrak{s u}^{*}(2)$, então sempre existe $H \in \mathcal{O}_{\xi}$ tal que $H=i \operatorname{diag}(\lambda,-\lambda)$. Vamos considerar $\xi \neq 0$, assim $\lambda \neq 0$, afim de verificar se a ação é livre, precisamos checar se existe $g \in S U(2)-\{I\}$ tal que

$$
\operatorname{Ad}_{g}^{*} H=H
$$

É importante salientar que é suficiente estudar se a ação é livre em $H$, pois uma vez sabendo qual o subgrupo de isotropia de $H$, saberemos o subgrupo de isotropia de todos os demais pontos de $\mathcal{O}_{\xi}$, onde $H \in \mathcal{O}_{\xi}$, mais precisamente, o estabilizador pertence a classe de conjugação de $S U(2)_{H}$. Pela equivalência das representações adjunta e coadjunta, basta ver se ocorre

$$
g H g^{-1}=H \text { ou seja } g H=H g,
$$

sendo $g \in S U(2)$, temos que existem $a, b \in \mathbb{C}$ tais que

$$
g=\left(\begin{array}{cc}
a & -\bar{b} \\
b & \bar{a}
\end{array}\right),|a|^{2}+|b|^{2}=1
$$


assim,

$$
\left(\begin{array}{cc}
a & -\bar{b} \\
b & \bar{a}
\end{array}\right) \cdot\left(\begin{array}{cc}
i \lambda & 0 \\
0 & -i \lambda
\end{array}\right)=\left(\begin{array}{cc}
i \lambda & 0 \\
0 & -i \lambda
\end{array}\right) \cdot\left(\begin{array}{cc}
a & -\bar{b} \\
b & \bar{a}
\end{array}\right)
$$

fazendo as contas e levando em consideração que tomamos $\xi \neq 0, \operatorname{logo} \lambda \neq 0$, obtemos

$$
\left(\begin{array}{cc}
a & \bar{b} \\
b & -\bar{a}
\end{array}\right)=\left(\begin{array}{cc}
a & -\bar{b} \\
-b & -\bar{a}
\end{array}\right)
$$

desta última e do fato que $|a|^{2}+|b|^{2}=1$, obtemos $b=0$ e $|a|^{2}=1$, portanto

$$
g=\left(\begin{array}{cc}
a & 0 \\
0 & \bar{a}
\end{array}\right)
$$

fazendo a correspondência $\mathfrak{s u}(2) \simeq \mathbb{R}^{3}$, temos que o subgrupo de isotropia de $v_{H}=(\lambda, 0,0)$ é

$$
S U(2)_{v_{H}}=\left\{\left.g \in S U(2)\left|g=\left(\begin{array}{cc}
a & 0 \\
0 & \bar{a}
\end{array}\right),\right| a\right|^{2}=1\right\}
$$

assim, concluímos que a ação não é livre em $H$, ou seja, em toda órbita coadjunta existe ao menos um ponto que é estabilizado por este subgrupo, e ainda mais, pelo resultado que diz que os pontos de uma mesma órbita possuem estabilizadores em uma mesma classe de conjugação, segue que a ação não é livre em nenhum ponto da órbita $\mathcal{O}_{\xi} \subset \mathfrak{s u}^{*}(2)$ e desde que

$$
\mathfrak{s u}^{*}(2)=\bigsqcup_{\xi \in \mathfrak{s u}^{*}(2)} \mathcal{O}_{\xi}
$$

temos que a ação coadjunta de $S U(2)$ em $\mathfrak{s u}^{*}(2)$ não é livre em nenhum ponto de $\mathfrak{s u}^{*}(2)$.

Agora faremos $S U(2) \curvearrowright\left(\mathfrak{s u}^{*}(2)\right)^{n}$ para algum $n \in \mathbb{N}$ fixado, com a ação sendo a coadjunta de forma diagonal ${ }^{3}$, a qual denotaremos por $\mathbf{A d}^{*}$ em negrito. Agora, pela equivalência das representações, note que estudar $S U(2) \curvearrowright\left(\mathfrak{s u}^{*}(2)\right)^{n}$ é equivalente a estudar $S U(2) \curvearrowright(\mathfrak{s u}(2))^{n}$, daí

$$
\begin{aligned}
\mathbf{A d}: S U(2) \times \prod_{j=1}^{n} \mathfrak{s u}(2) & \longrightarrow \prod_{j=1}^{n} \mathfrak{s u}(2) \\
(g, X) & \longmapsto \operatorname{Ad}_{g} X=\left(\operatorname{Ad}_{g} X_{1}, \ldots, \operatorname{Ad}_{g} X_{n}\right)
\end{aligned}
$$

\footnotetext{
${ }^{3}$ Uma ação diagonal de um grupo de Lie $G$ sobre a variedade produto $M_{1} \times M_{2}$ é definida por

$$
g \cdot\left(m_{1}, m_{1}\right)=\left(g \cdot m_{1}, g \cdot m_{2}\right), m_{1} \in M_{1}, m_{2} \in M_{2}, \forall g \in G
$$
}


onde $X=\left(X_{1}, \ldots, X_{n}\right) \in \prod_{j=1}^{n} \mathfrak{s u}(2)$. Ou seja, $S U(2)$ age em cada fator $\mathfrak{s u}(2)$ do produto cartesiano $\prod_{j=1}^{n} \mathfrak{s u}(2)$ pela ação adjunta.

Sabemos que para cada $X_{j} \in \mathfrak{s u}(2)$ existe um $\lambda_{j} \in \mathbb{R}$ e $g \in S U(2)$ tal que

$$
\operatorname{Ad}_{g} X_{j}=\operatorname{diag}\left(i \lambda_{j},-i \lambda_{j}\right), j=1, \ldots, n
$$

dessa forma, pelo o que mostramos anteriormente, a órbita de $S U(2)$ por $X_{j}$ é a 2 -esfera $\mathbb{S}_{\left|\lambda_{j}\right|}^{2}$, consequentemente a órbita coadjunta diagonal de $S U(2)$ por $X$ é dada por

$$
\mathcal{O}_{X}^{\mathcal{D}}=\mathcal{D}_{X}\left(\prod_{j=1}^{n} \mathcal{O}_{X_{j}}\right) \simeq \mathcal{D}_{X}\left(\prod_{j=1}^{n} \mathbb{S}_{\left|\lambda_{j}\right|}^{2}\right)=\mathcal{S}_{r}
$$

onde $r=\left(r_{1}, \ldots, r_{n}\right) \in \mathbb{R}_{+}^{n}$ de tal forma que $r_{j}=\left|\lambda_{j}\right|$ para todo $j$. A notação $\mathcal{O}_{X}^{\mathcal{D}}$ significa que $X \in \mathcal{O}_{X}^{\mathcal{D}}$ e para todo $Y \in \mathcal{O}_{X}^{\mathcal{D}}$ existe $g \in S U(2)$ tal que $\operatorname{Ad}_{g} X=\left(\operatorname{Ad}_{g} X_{1}, \ldots, \operatorname{Ad}_{g} X_{n}\right)=Y$.

Note que em $\mathcal{S}_{r}$ pode não existir o ponto $Y$ onde todas as entradas de $Y$ são da forma $Y_{j}=i \operatorname{diag}\left(\lambda_{j},-\lambda_{j}\right)$, esse ponto estará em $\mathcal{S}_{r}$ somente se todas as entradas de $X$ podem ser diagonalizadas pelo mesmo $g \in S U(2)$, mais precisamente, se $Y_{j}=g X_{j} g^{-1}$ para todo $j$.

Agora considere a restrição da ação $\left.\mathbf{A d}\right|_{S U(2) \times \mathcal{S}_{r}}$ a qual é Hamiltoniana com aplicação momento ${ }^{4}$ dada por

$$
\begin{aligned}
\mu: \mathcal{S}_{r} & \longrightarrow \mathfrak{s u}^{*}(2) \\
\xi=\left(\xi_{1}, \ldots, \xi_{n}\right) & \longmapsto \mu(\xi)=\sum_{j=1}^{n} \xi_{j} .
\end{aligned}
$$

Para $0 \in \mathfrak{s u}^{*}(2)$, o conjunto de nível $\mu^{-1}(0)$ é um subconjunto de $\mathcal{S}_{r}$ dado por

$$
\mu^{-1}(0):=\widetilde{M}_{r}=\left\{\xi=\left(\xi_{1}, \ldots, \xi_{n}\right) \in \mathcal{S}_{r} \mid \sum_{j=1}^{n} \xi_{j}=0\right\},
$$

onde $r=\left(r_{1}, \ldots, r_{n}\right) \in \mathbb{R}_{+}^{n}$, sendo $r_{j}$ o raio da esfera $\mathbb{S}_{r_{j}}^{2}$ para cada $j$, segue que $\xi_{j} \in \mathbb{S}_{r_{j}}^{2}$ e assim $\left\|\xi_{j}\right\|=r_{j}$.

Observação 5.1 Um polígono em $\mathbb{R}^{3}$ é um caminho linear por parte fechado em $\mathbb{R}^{3}$. Agora se consideramos um caminho linear por parte, tal que o j-ésimo

\footnotetext{
${ }^{4}$ Quando um grupo de Lie $G$ age em duas variedades simpléticas $\left(M_{j}, \omega_{j}\right), j=1,2$, com aplicações momento $\mu_{j}: M_{j} \rightarrow \mathfrak{g}^{*}$, a ação diagonal de $G$ em $M_{1} \times M_{2}$ tem aplicação momento $\mu: M_{1} \times M_{2} \rightarrow \mathfrak{g}^{*}, \mu\left(p_{1}, p_{2}\right)=\mu_{1}\left(p_{1}\right)+\mu_{2}\left(p_{2}\right)$.
} 
passo é dado pelo vetor $\xi_{j}$. Um tal caminho se fecha se, e somente se, $\sum_{j=1}^{n} \xi_{j}=0$. Assim, $\widetilde{M}_{r}$ é o espaço dos $n$-gons de lados $\xi_{j}$ de comprimentos fixados $r_{j}$.

Notamos agora, que dado $\xi \in \mu^{-1}(0)$ é possível que exista um $\tilde{\xi} \in \mu^{-1}(0)$ tal que

$$
\tilde{\xi}=g \xi g^{-1}=\operatorname{Ad}_{g} \xi
$$

para algum $g \in S U(2)$, afim de termos $\xi$ e $\tilde{\xi}$ em uma mesma classe, tomamos o quociente

$$
M_{r}=\widetilde{M}_{r} / S U(2)=\mu^{-1}(0) / S U(2)=\mathcal{S}_{r} / / S U(2)=\mathcal{D}_{X}\left(\prod_{j=1}^{n} \mathbb{S}_{r_{j}}^{2}\right) / /_{0} S U(2),
$$

é o espaço dos $n$-gons de comprimento de lados fixado $r_{1}, \ldots, r_{n}$, módulo movimento rígido, e é chamado de espaço de polígonos. Uma vez que a ação de $S U(2)$ em $\widetilde{M}_{r}=\mu^{-1}(0)$ não é livre, logo o quociente acima tem singularidades, resta analisar quais são essas singularidades, sabe-se que essas singularidades isoladas correspondem aos $n$-gons degenerados em $M_{r}$, para mais informações o prezado leitor pode consulta [9] página 8.

Dizemos que um polígono é degenerado se estiver completamente em uma linha. Uma forma de saber se o quociente $M_{r}$ tem singularidades, é analisando o vetor de comprimento $r$, por meio da seguinte definição.

Definição 5.2 Um vetor de comprimento $r=\left(r_{1}, \ldots, r_{n}\right)$ é chamado genérico se não existe um subconjunto $I \subset\{1, \ldots, n\}$ de modo que a quantidade

$$
\epsilon_{I}(r):=\sum_{i \in I} r_{i}-\sum_{i \in I^{c}} r_{i}
$$

é nula

O espaço $M_{r}$ é uma variedade suave se, e somente se, o vetor de comprimento $r$ é genérico. Equivalentemente, se, e somente se, em $M_{r}$ não existem polígonos degenerados.

Podemos montar um diagrama que relaciona $\mathcal{S}_{r}, \widetilde{M}_{r}$ e os quocientes $\mathcal{S}_{r} / S U(2)$ e $M_{r}=\mu^{-1}(0) / S U(2)$

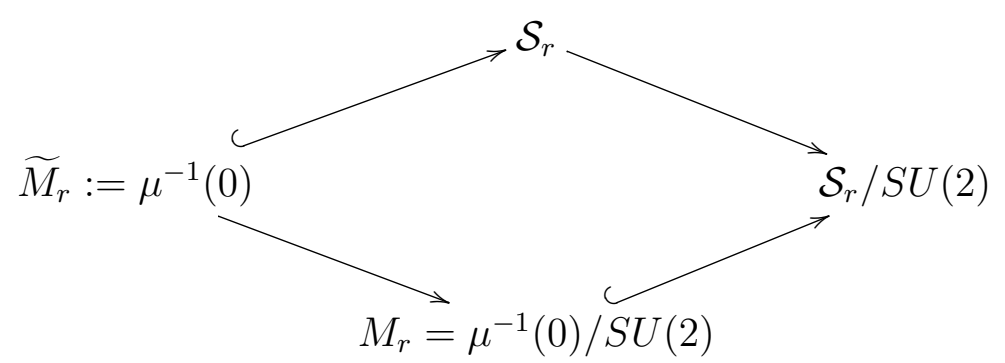


Mostramos anteriormente que a ação coadjunta de $S U(2)$ em $\mathfrak{s u}^{*}(2)$ não é livre, vamos agora investigar a ação adjunta diagonal de $S U(2)$ em $\mathcal{S}_{r}$. Lembrando que

$$
\mathcal{O}_{X}^{\mathcal{D}}=\mathcal{D}_{X}\left(\prod_{j=1}^{n} \mathcal{O}_{X_{j}}\right) \simeq \mathcal{D}_{X}\left(\prod_{j=1}^{n} \mathbb{S}_{\left|\lambda_{j}\right|}^{2}\right)=\mathcal{S}_{r}
$$

dessa forma, é suficiente verificar se o estabilizador de $X$ é trivial, uma vez que o estabilizador de $Y \in \mathcal{S}_{r}$ é dado por $S U(2)_{Y}=g S U(2)_{X} g^{-1}$, onde $g$ satisfaz

$$
\operatorname{Ad}_{g} X=\left(\operatorname{Ad}_{g} X_{1}, \ldots, \operatorname{Ad}_{g} X_{n}\right)=Y,
$$

ou seja, sabendo o estabilizador de $X$ saberemos de todos os demais pontos de $\mathcal{S}_{r}$.

Considere a possibilidade em que $X=\left(X_{1}, \ldots, X_{n}\right)$ onde todas as entradas $X_{j}$ são da forma

$$
X_{j}=\left(\begin{array}{cc}
i x_{j} & 0 \\
0 & -i x_{j}
\end{array}\right), x_{j} \in \mathbb{R} \forall j
$$

esse ponto é estabilizado pela ação adjunta diagonal por elementos $g \in S U(2)$ da forma

$$
g=\left(\begin{array}{cc}
m & 0 \\
0 & \bar{m}
\end{array}\right), m \in \mathbb{C}, \text { onde }|m|^{2}=1
$$

de fato,

$$
\left(\begin{array}{cc}
m & 0 \\
0 & \bar{m}
\end{array}\right) \cdot\left(\begin{array}{cc}
i x_{j} & 0 \\
0 & -i x_{j}
\end{array}\right) \cdot\left(\begin{array}{cc}
\bar{m} & 0 \\
0 & m
\end{array}\right)=|m|^{2}\left(\begin{array}{cc}
i x_{j} & 0 \\
0 & -i x_{j}
\end{array}\right)=X_{j}
$$

note que a conta acima independe de $x_{j}$, daí

$$
\operatorname{Ad}_{g} X_{j}=g X_{j} g^{-1}=X_{j}, j=1, \ldots, n
$$

note também que todos os pontos da forma

$$
A=\left\{\left(X_{1}, \ldots, X_{n}\right),\left(X_{1}, \ldots, X_{n-1}, 0\right), \ldots,\left(X_{1}, 0, \ldots, 0\right)\right\}
$$

onde $X_{j}=\operatorname{diag}\left(i x_{j},-i x_{j}\right)$, ou qualquer ponto que seja uma permutação das entradas desses pontos, também são estabilizados pelo subgrupo

$$
S U(2)_{x_{j}}=\left\{g \in S U(2)\left|g=\left(\begin{array}{cc}
m & 0 \\
0 & \bar{m}
\end{array}\right),\right| m \mid=1, m \in \mathbb{C}\right\} .
$$


portanto se $X$ é da forma de um dos pontos do conjunto $A$, a menos de uma permutação, então o estabilizador de $X$ não é trivial e a ação adjunta diagonal de $S U(2)$ em $\mathcal{S}_{r}$ não é livre.

Considere agora a possibilidade em que $X=\left(X_{1}, \ldots, X_{n}\right)$ onde todas as entradas $X_{j}$ são da forma

$$
X_{j}=\left(\begin{array}{cc}
0 & y_{j} \\
-y_{j} & 0
\end{array}\right), y_{j} \in \mathbb{R} \forall j
$$

esse ponto é estabilizado pela ação adjunta diagonal por elementos $g \in S U(2)$ da forma

$$
g=\left(\begin{array}{cc}
m & -k \\
k & m
\end{array}\right), m, k \in \mathbb{R}, m^{2}+k^{2}=1
$$

de fato

$$
\begin{aligned}
\left(\begin{array}{cc}
m & -k \\
k & m
\end{array}\right) \cdot\left(\begin{array}{cc}
0 & y_{j} \\
-y_{j} & 0
\end{array}\right) \cdot\left(\begin{array}{cc}
m & k \\
-k & m
\end{array}\right) & =\left(k^{2}+m^{2}\right)\left(\begin{array}{cc}
0 & y_{j} \\
-y_{j} & 0
\end{array}\right) \\
& =\left(\begin{array}{cc}
0 & y_{j} \\
-y_{j} & 0
\end{array}\right)
\end{aligned}
$$

como esta conta independe de $y_{j}$, segue que

$$
\operatorname{Ad}_{g} X_{j}=g X_{j} g^{-1}=X_{j}, j=1, \ldots, n
$$

note que todos os pontos da forma

$$
B=\left\{\left(X_{1}, \ldots, X_{n}\right),\left(X_{1}, \ldots, X_{n-1}, 0\right), \ldots,\left(X_{1}, 0, \ldots, 0\right)\right\}
$$

onde

$$
X_{j}=\left(\begin{array}{cc}
0 & y_{j} \\
-y_{j} & 0
\end{array}\right), y_{j} \in \mathbb{R} \forall j
$$

ou qualquer ponto que seja uma permutação das entradas desses pontos, são estabilizados pelo subgrupo

$$
S U(2)_{y_{j}}=\left\{g \in S U(2) \mid g=\left(\begin{array}{cc}
m & -k \\
k & m
\end{array}\right), m^{2}+k^{2}=1, m, k \in \mathbb{R}\right\} .
$$

portanto se $X$ é da forma de um dos pontos do conjunto $B$, a menos de uma permutação, então o estabilizador de $X$ não é trivial e a ação adjunta diagonal de $S U(2)$ em $\mathcal{S}_{r}$ não é livre.

Considere agora a possibilidade em que $X=\left(X_{1}, \ldots, X_{n}\right)$ onde todas as 
entradas $X_{j}$ são da forma

$$
X_{j}=\left(\begin{array}{cc}
0 & i z_{j} \\
i z_{j} & 0
\end{array}\right), z_{j} \in \mathbb{R} \forall j
$$

esse ponto é estabilizado pela ação adjunta diagonal por elementos $g \in S U(2)$ da forma

$$
g=\left(\begin{array}{cc}
m & i k \\
i k & m
\end{array}\right), m, k \in \mathbb{R}, m^{2}+k^{2}=1
$$

de fato

$$
\begin{aligned}
\left(\begin{array}{cc}
m & i k \\
i k & m
\end{array}\right) \cdot\left(\begin{array}{cc}
0 & i z_{j} \\
i z_{j} & 0
\end{array}\right) \cdot\left(\begin{array}{cc}
m & -i k \\
-i k & m
\end{array}\right) & =\left(k^{2}+m^{2}\right)\left(\begin{array}{cc}
0 & i z_{j} \\
i z_{j} & 0
\end{array}\right) \\
& =\left(\begin{array}{cc}
0 & i z_{j} \\
i z_{j} & 0
\end{array}\right)
\end{aligned}
$$

note que a conta acima independe de $z_{j}$, daí

$$
\operatorname{Ad}_{g} X_{j}=g X_{j} g^{-1}=X_{j}, j=1, \ldots, n
$$

dessa forma, todos os pontos da forma

$$
C=\left\{\left(X_{1}, \ldots, X_{n}\right),\left(X_{1}, \ldots, X_{n-1}, 0\right), \ldots,\left(X_{1}, 0, \ldots, 0\right)\right\}
$$

onde

$$
X_{j}=\left(\begin{array}{cc}
0 & i z_{j} \\
i z_{j} & 0
\end{array}\right), z_{j} \in \mathbb{R} \forall j
$$

ou qualquer ponto que seja uma permutação das entradas desses pontos, são estabilizados pelo subgrupo

$$
S U(2)_{z_{j}}=\left\{g \in S U(2) \mid g=\left(\begin{array}{cc}
m & i k \\
i k & m
\end{array}\right), m^{2}+k^{2}=1, m, k \in \mathbb{R}\right\},
$$

portanto se $X$ é da forma de um dos pontos do conjunto $C$, a menos de uma permutação, então o estabilizador de $X$ não é trivial e a ação adjunta diagonal de $S U(2)$ em $\mathcal{S}_{r}$ não é livre.

Exibimos o estabilizador em casos particulares para $X$, agora se $X$ não se enquadra em nenhum dos três casos anteriores, ainda assim a ação não será livre, uma vez que para qualquer $X=\left(X_{1}, \ldots, X_{n}\right)$ com $X_{j} \in \mathfrak{s u}(2), X$ sempre será estabilizado por

$$
g= \pm\left(\begin{array}{ll}
1 & 0 \\
0 & 1
\end{array}\right)
$$


portanto a ação adjunta diagonal de $S U(2)$ em $\mathcal{S}_{r}$ não é livre.

Agora note que se pensarmos em fazer $\sum_{j=1}^{n} X_{j}=0$, usando a correspondência $\mathfrak{s u}^{*}(2) \simeq \mathbb{R}^{3}$, estaremos pensando em $n$ vetores em $\mathbb{R}^{3}$ tal que a soma deles é igual a zero, ou seja, um polígono em $\mathbb{R}^{3}$, no entanto, no primeiro caso temos que os $n$ vetores $v_{X_{j}}$ são todos da forma

$$
v_{X_{j}}=x_{j}(1,0,0)=x_{j} e_{1}
$$

para o segundo caso

$$
v_{X_{j}}=y_{j}(0,1,0)=y_{j} e_{2}
$$

enquanto que para o terceiro caso

$$
v_{X_{j}}=z_{j}(0,0,1)=z_{j} e_{3}
$$

logo, pensar em $\sum_{j=1}^{n} X_{j}=0$, seria pensar nos três casos em polígonos degenerados uma vez que estão totalmente contidos nos eixos $O_{x}, O_{y}$ e $O_{z}$, respectivamente. 


\section{$S U(n)$ e suas órbitas coadjunta}

Neste capítulo trabalhamos com grupos de Lie de matrizes, fornecemos alguns exemplos, abordamos grupos conexos e compactos, mostramos que o grupo $S U(n)$ é compacto. Enunciamos e demonstramos o resultado que trata a respeito de ação contínua de grupo de Lie compacto ser uma ação própria. Fazemos uso da exponencial de matrizes a qual será muito útil para compreender os cálculos das álgebras de Lie $\mathfrak{o}(n), \mathfrak{u}(n)$ e $\mathfrak{s u}(n)$.

$\mathrm{Na}$ segunda parte apresentamos um resultado muito importante que diz que a órbita adjunta de $S U(n)$ por $X \in \mathfrak{s u}(n)$ é difeomórfica a uma determinada variedade bandeira complexa.

$\mathrm{Na}$ terceira parte estudamos ação adjunta diagonal de $S U(n)$ na variedade $\prod_{j=1}^{m} \mathfrak{s u}(n)$, e finalmente na última seção vemos que o toro estabiliza os elementos da base de $\mathfrak{s u}(n)$.

\section{1}

Grupos de Lie de matrizes e as álgebras de Lie $\mathfrak{o}(n), \mathfrak{u}(n)$ e $\mathfrak{s u}(n)$

Seja $M_{n}(\mathbb{C})$ o espaço das matrizes $n \times n$ com entradas complexas. Podemos identificar $M_{n}(\mathbb{C})$ com $\mathbb{C}^{n^{2}}$ e usar a noção padrão de convergência em $\mathbb{C}^{n^{2}}$. Explicitamente, isto significa o seguinte.

Definição 6.1 Seja $A_{m}$ uma sequência de matrizes complexas em $M_{n}(\mathbb{C})$. Dizemos que $A_{m}$ converge para a matriz $A$ se cada entrada de $A_{m}$ converge para a entrada correspondente de $A$, isto é, se $\left(A_{m}\right)_{j k}$ converge para $A_{j k}$ para todo $1 \leq j, k \leq n$.

Seja $G L(n, \mathbb{C})$ o grupo linear geral complexo o qual é formado pelas matrizes invertíveis $n \times n$ com entradas em $\mathbb{C}$. Consideramos agora subgrupos de $G L(n, \mathbb{C})$, isto é, subconjuntos $G$ de $G L(n, \mathbb{C})$ tal que a matriz identidade está em $G$ e tal que para todo $A$ e $B$ em $G$, as matrizes $A B$ e $A^{-1}$ estão também em $G$.

Definição 6.2 Um grupo de Lie de matrizes é um subgrupo $G$ de $G L(n, \mathbb{C})$ com a seguinte propriedade: Se $A_{m}$ é uma sequência de matrizes em $G$, e $A_{m}$ converge para alguma matriz $A$, então $A$ está em $G$ ou $A$ não é invertível. 
A condição sobre $G$ é que este seja um subconjunto fechado de $G L(n, \mathbb{C})$, isto não significa que $G$ é fechado em $M_{n}(\mathbb{C})$. Assim, essa definição é equivalente a dizer que um grupo de Lie de matrizes é um subgrupo fechado de $G L(n, \mathbb{C})$.

Exemplo 6.3 Em [22] são listados vários exemplos, vejamos alguns que sejam ou não grupos de Lie de matrizes.

(a) O grupo $G L(n, \mathbb{K}), \mathbb{K}=\mathbb{R}$ ou $\mathbb{C}$, das matrizes invertiveis $n \times n$ com entradas em $\mathbb{K}$, claramente é um grupo de Lie de matrizes.

(b) O grupo especial linear $S L(n, \mathbb{K}), \mathbb{K}=\mathbb{R}$ ou $\mathbb{C}$, das matrizes invertíveis $n \times n$ com determinante um, claramente é subgrupo fechado de $G L(n, \mathbb{K})$, pois o determinante é uma função contínua, logo é um grupo de Lie de matrizes.

(c) O grupo das matrizes unitárias, $U(n)=\left\{A \in G L(n, \mathbb{C}) \mid A A^{*}=I=A^{*} A\right\}$, $e$ o grupo especial unitário, $S U(n)=\{A \in U(n) \mid \operatorname{det}(A)=1\}$, são subgrupos fechados de $G L(n, \mathbb{C})$ e portanto são grupos de Lie de matrizes.

(a) Considere o subgrupo de $G L(n, \mathbb{C})$ formado por todas as matrizes $n \times n$ invertíveis com entradas racionais. Este não é um grupo de Lie de matrizes pois não é fechado em $G L(n, \mathbb{C})$, isto se deve ao fato de que toda matriz invertível real é limite de alguma sequência de matrizes invertíveis com entradas racionais.

Definição 6.4 Um grupo de Lie de matrizes $G \subset G L(n, \mathbb{C})$ é dito ser compacto se é compacto no sentido usual da topologia como um subconjunto de $M_{n}(\mathbb{C}) \cong \mathbb{R}^{2 n^{2}}$.

Observação 6.5 Um grupo de Lie de matrizes $G$ é compacto se, e somente se, é fechado como subconjunto de $M_{n}(\mathbb{C})$, não apenas como subconjunto de $G L(n, \mathbb{C})$ e limitado. Explicitamente, isto significa que $G$ é compacto se, e somente se,

1. sempre que $A_{m} \in G$ e $A_{m} \rightarrow A$, então A está em $G$, e

2. existe uma constante $C$ tal que para todo $A \in G$, temos $\left|A_{j k}\right| \leq C$ para todo $1 \leq j, k \leq n$.

Exemplo 6.6 Os seguintes grupos são compactos: $O(n), S O(n), U(n)$ e $S U(n)$. 
Não iremos provar que estes grupos são compactos, faremos isso apenas para o $S U(n)$, o qual utilizamos bastante, $S U(n)$ é limitado, pois

$$
\left|A_{i j}\right|^{2} \leq \sum_{j=1}^{n}\left|A_{i j}\right|^{2}=\left(A e_{i}, A e_{i}\right)=1, \text { para } A \in S U(n),
$$

isto se deve ao fato de que as colunas de $A \in S U(n)$ devem ser vetores unitários. Onde estamos considerando o espaço vetorial complexo $n$ dimensional $\mathbb{C}^{n}$ com produto interno hermitiano,

$$
(x, y)=\sum_{i=1}^{n} \overline{x_{i}} y_{i}
$$

onde $x=\left(x_{1}, \ldots, x_{n}\right), y=\left(y_{1}, \ldots, y_{n}\right) \in \mathbb{C}^{n}$ e $\bar{x}$ denota o conjugado complexo de $x$. O grupo especial unitário $S U(n)$ é então dado por

$$
S U(n)=\{A \in G L(n, \mathbb{C}) \mid(A x, A y)=(x, y), \operatorname{det}(A)=1\}\} .
$$

equivalentemente

$$
\left.S U(n)=\left\{A \in G L(n, \mathbb{C}) \mid A \cdot A^{*}=A^{*} \cdot A=I, \operatorname{det}(A)=1\right\}\right\},
$$

onde $A^{*}$ denota o conjugado transposto de $A$, isto é, $A^{*}=(\bar{A})^{T}$. Para ver que $S U(n)$ é fechado, usamos o fato de que as funções

$$
A \mapsto A^{*} A \text { e } A \mapsto \operatorname{det}(A)
$$

são funções contínuas. Agora considere a aplicação

$$
\begin{aligned}
f: M_{n}(\mathbb{C}) & \longrightarrow M_{n}(\mathbb{C}) \times \mathbb{C} \\
A & \longmapsto f(A)=\left(A^{*} A, \operatorname{det}(A)\right)
\end{aligned}
$$

note que $f$ é contínua, assim, a pré-imagem do conjunto fechado $\{(\mathbf{1}, 1)\}$ pela aplicação $f$ é um conjunto fechado, aqui 1 denota a matriz identidade $n \times n$. Mas $f^{-1}(\{\mathbf{1}, 1\})=S U(n)$, provando assim que $S U(n)$ é um subconjunto fechado de $G L(n, \mathbb{C})$. Concluímos assim que $S U(n)$ é compacto.

Definição 6.7 Um grupo de Lie de matrizes $G$ é dito ser conexo se para todo $A$ e $B$ em $G$, existe um caminho contínuo $A(t), a \leq t \leq b$, em $G$ com $A(a)=A$ e $A(b)=B$. Para qualquer grupo de Lie de matrizes $G$, a componente da identidade de $G$, denotada por $G_{0}$, é o conjunto de $A \in G$ para o qual existe um caminho contínuo $A(s), a \leq s \leq b$, em $G \operatorname{com} A(a)=I$ e $A(b)=A$. 
Portanto para mostrar que um grupo de Lie de matrizes $G$ é conexo, é suficiente mostrar que cada $A \in G$ pode ser conectado a identidade por com caminho contínuo em $G$.

Proposição 6.8 Os grupos $U(n)$ e $S U(n)$ são conexos, para todo $n \geq 1$.

A demonstração desta proposição o prezado leitor pode encontrar em [22], Proposição 1.13.

O próximo resultado nos fornece uma caracterização das ações próprias de um grupo de Lie em uma variedade, a demonstração desse resultado o prezado leitor pode encontrar em [14], Proposição 21.5.

Proposição 6.9 Sejam $M$ uma variedade e G um grupo de Lie agindo continuamente em $M$. Então as afirmações a seguir são equivalentes.

1. A ação é própria.

2. Se $\left(p_{i}\right)$ é uma sequência em $M$ e $\left(g_{i}\right)$ uma sequência em $G$ tal que $\left(p_{i}\right)$ e $\left(g_{i} \cdot p_{i}\right)$ convergem, então uma subsequência de $\left(g_{i}\right)$ converge.

3. Para todo subconjunto compacto $K \subseteq M$, o conjunto

$$
G_{K}=\{g \in G \mid(g \cdot K) \cap K \neq \emptyset\}
$$

é compacto.

Corolário 6.10 Toda ação contínua de um grupo de Lie compacto em uma variedade é própria.

Proof. Sejam $\left(p_{i}\right)$ e $\left(g_{i}\right)$ sequências como na proposição 6.9, então uma subsequência de $\left(g_{i}\right)$ converge, pela simples razão de que toda sequência em $G$ tem uma subsequência convergente, pois $G$ é compacto, assim, pela proposição 6.9 , segue o resultado.

Definição 6.11 (Caminhos suaves e suas derivadas) Seja $S$ um espaço de matrizes. Dizemos que um caminho $t \mapsto A(t)$ é suave ou diferenciável, se as funções coordenadas $a_{i j}(t)$ são diferenciáveis. Se $A(t)$ é suave, sua derivada $A^{\prime}(t)$ é definida da forma usual por

$$
A^{\prime}(t)=\lim _{\Delta t \rightarrow 0} \frac{A(t+\Delta t)-A(t)}{\Delta t}
$$

Segue-se a partir da definição que $A^{\prime}(t)$ é simplesmente a matriz com entradas $a_{i j}^{\prime}(t)$ onde $a_{i j}$ são as entradas de $A(t)$. 
Definição 6.12 (Vetores tangentes e o espaço tangente) Os vetores tangentes na identidade $I$ de um grupo de Lie de matrizes $G$ são as matrizes $X$ da forma

$$
X=A^{\prime}(0)
$$

onde $A(t)$ é um caminho suave em $G$ com $A(0)=I$. O espaço tangente a $G$ na identidade $I$ é o conjunto de vetores tangentes em $I$.

Proposição 6.13 (Exponenciação de vetores tangentes) Se $X$ é um vetor tangente na identidade para um grupo de Lie de matrizes $G$, então $e^{X} \in G$. Em outras palavras, exp mapeia o espaço tangente $T_{I} G$ em $G$.

\section{Corolário 6.14 (Uma descrição alternativa da álgebra de Lie)}

Suponha que $G \subseteq G L(n, \mathbb{C})$ é um grupo de Lie de matrizes com álgebra de Lie $\mathfrak{g}$. Então $X \in M_{n}(\mathbb{C})$ está em $\mathfrak{g}$ se, e somente se, $e^{t X} \in G$ para todo $t \in \mathbb{R}$.

Observação 6.15 Vimos na Proposição 6.13 que se $X$ é um vetor tangente na identidade para o grupo de Lie de matrizes $G$, então $e^{X} \in G$. E o Corolário 6.14 deu uma definição equivalente de espaço tangente, como sendo o conjunto de todos os $X$ tal que $e^{t X} \in G \forall t \in \mathbb{R}$.

Se $X$ é uma matriz $n \times n$, definimos a exponencial de $X$, denotada por $e^{X}$ ou $\exp (X)$, pela série de potência

$$
e^{X}=\sum_{j=0}^{\infty} \frac{X^{j}}{j !}
$$

onde $X^{0}$ é definida como a matriz identidade $I$ e $X^{j}$ representa o produto de $X$ por $X j-1$ vezes.

O resultado a seguir nos fornece algumas propriedades referente a exponencial de matrizes.

Proposição 6.16 Sejam $X$ e $Y$ matrizes $n \times n$. Então temos:

$$
\begin{aligned}
& \text { 1. }\left(e^{X}\right)^{*}=e^{X^{*}} \\
& \text { 2. } e^{(\alpha+\beta) X}=e^{\alpha X} \cdot e^{\beta X} \forall \alpha, \beta \in \mathbb{C} . \\
& \text { 3. Se } X Y=Y X \text {, entãa } e^{X+Y}=e^{X} \cdot e^{Y}=e^{Y} \cdot e^{X} \\
& \text { 4. } e^{X} \text { é invertível } e\left(e^{X}\right)^{-1}=e^{-X}
\end{aligned}
$$


Proposição 6.17 Seja X uma matriz complexa quadrada. Então $t \mapsto e^{t X} e^{\prime}$ uma curva suave em $M_{n}(\mathbb{C})$ e

$$
\frac{d}{d t} e^{t X}=X \cdot e^{t X}=e^{t X} \cdot X
$$

em particular

$$
\left.\frac{d}{d t} e^{t X}\right|_{t=0}=X
$$

Proposição 6.18 Para qualquer $X \in M_{n}(\mathbb{C})$, temos $\operatorname{det}\left(e^{X}\right)=e^{\operatorname{tr}(X)}$

A demonstração destes três resultados o prezado leitor encontra em [22], Proposição 2.3, 2.4 e Teorema 2.12.

Corolário 6.19 Suponha que $A(t)$ é um caminho suave em $M_{n}(\mathbb{C})$. Então

$$
\frac{d}{d t} A(t)^{T}=\left(\frac{d}{d t} A(t)\right)^{T} \quad e \frac{d}{d t} \overline{A(t)}=\overline{A^{\prime}(t)}
$$

onde o $T$ no expoente denota a transposta.

Proposição 6.20 O espaço tangente na identidade dos grupos $O(n)$ e $U(n)$ são dados por

$$
\mathfrak{o}(n)=\left\{X \in M_{n}(\mathbb{R}) \mid X+X^{T}=0\right\} \text { e } \mathfrak{u}(n)=\left\{X \in M_{n}(\mathbb{C}) \mid X+X^{*}=0\right\}
$$

respectivamente, onde $X^{*}=\bar{X}^{T}$, isto é, $X^{*}$ representa a matriz transposta conjugada de $X$.

Proof. Lembramos inicialmente que

$$
\begin{aligned}
& O(n)=\left\{A \in G L(n, \mathbb{R}) \mid A \cdot A^{T}=I=A^{T} \cdot A\right\} \\
& U(n)=\left\{A \in G L(n, \mathbb{C}) \mid A \cdot A^{*}=I=A^{*} \cdot A\right\}
\end{aligned}
$$

Faremos a demonstração da seguinte forma, mostraremos que o espaço tangente na identidade de $O(n)$ e $U(n)$ está contido em $\mathfrak{o}(n)$ e $\mathfrak{u}(n)$, respectivamente, e depois faremos a inclusão inversa, provando assim o resultado.

Seja $A(t)$ um caminho suave em $O(n)$ ou $U(n)$ com $A(0)=I$. Então

$$
A(t) \cdot A(t)^{*}=I
$$


para todo $t$, caso $A(t) \in O(n)$, então $A(t)^{*}=A(t)^{T}$. Do Corolário 6.19, derivando (6.1) com respeito a $t$ obtemos

$$
A^{\prime}(t) \cdot A(t)^{*}+A(t) \cdot A^{\prime}(t)^{*}=0
$$

avaliando em $t=0$, como $A(0)=I, \operatorname{logo} A(0)^{*}=I$, temos que

$$
A^{\prime}(0)+A^{\prime}(0)^{*}=0
$$

segue-se que os espaços tangentes estão contidos em $\mathfrak{o}(n)$ e $\mathfrak{u}(n)$. Note que estamos usando que $A^{*}=A^{T}$ se $A \in M_{n}(\mathbb{R})$.

Façamos agora a outra inclusão. Suponha que uma matriz $X$ satisfaz $X+X^{*}=0$. Então $e^{t X}$ é um caminho no grupo correspondente uma vez que

$$
\begin{array}{rlr}
e^{t X} \cdot\left(e^{t X}\right)^{*} & =e^{t X} \cdot e^{t X^{*}} & \\
& =e^{t X} \cdot e^{-t X} & \text { pois } X^{*}=-X \\
& =e^{t(X-X)} & \\
& =e^{0} & \\
& =I &
\end{array}
$$

e desde que

$$
\left.\frac{\mathrm{d}}{\mathrm{d} t} e^{t X}\right|_{t=0}=X
$$

vemos que $X$ está no espaço tangente, mostrando assim a outra inclusão. Portanto,

$$
\mathfrak{o}(n)=\left\{X \in M_{n}(\mathbb{R}) \mid X+X^{T}=0\right\} \text { e } \mathfrak{u}(n)=\left\{X \in M_{n}(\mathbb{C}) \mid X+X^{*}=0\right\}
$$

Definição 6.21 Seja $X \in M_{n}(\mathbb{R})$, dizemos que $X$ é anti-simétrica se $X+$ $X^{T}=0$. Se $X \in M_{n}(\mathbb{C})$, dizemos que $X$ é anti-hermitiana se $X+X^{*}=0$.

Sabemos que

$$
\begin{gathered}
U(n)=\left\{A \in G L(n, \mathbb{C}) \mid A \cdot A^{*}=I=A^{*} \cdot A\right\} \\
S U(n)=\{X \in U(n) \mid \operatorname{det} X=1\}
\end{gathered}
$$

ou equivalentemente

$$
S U(n)=\left\{X \in M_{n}(\mathbb{C}) \mid X^{*} \cdot X=X \cdot X^{*}=I, \operatorname{det} X=1\right\}
$$

Proposição 6.22 O espaço tangente de $S U(n)$ é dado por 


$$
\mathfrak{s u}(n)=\left\{X \in M_{n}(\mathbb{C}) \mid X+X^{*}=0, \operatorname{tr} X=0\right\}
$$

Proof. Sabemos que $S U(n) \subset U(n)$, dessa forma qualquer caminho em $S U(n)$ é também um caminho em $U(n)$ e assim o espaço tangente a identidade em $S U(n)$ está contido no espaço tangente a identidade em $U(n)$, assim o espaço tangente a $S U(n)$ satisfaz que

$$
X+X^{*}=0, \text { se } X \in T_{I} S U(n)
$$

isto é devido a Proposição 6.20. Portanto resta-nos mostrar que se $X \in$ $T_{I} S U(n)$ temos também

$$
\operatorname{tr} X=0
$$

É suficiente considerar caminhos da forma $t \mapsto e^{t X}$ afim de calcular o espaço tangente de $S U(n)$, isto é devido a Observação 6.15. Note que $t \mapsto e^{t X}$ é um caminho em $S U(n)$ se, e somente se,

$$
\begin{aligned}
\operatorname{det} e^{t X}=1 & \Leftrightarrow e^{t \cdot \operatorname{tr} X}=1 \forall t \\
& \Leftrightarrow t \cdot \operatorname{tr} X=0 \forall t \\
& \Leftrightarrow \operatorname{tr} X=0
\end{aligned}
$$

portanto

$$
\mathfrak{s u}(n)=\left\{X \in M_{n}(\mathbb{C}) \mid X+X^{*}=0, \operatorname{tr} X=0\right\}
$$

A partir de (6.3), podemos agora explicitar a forma dos elementos de $\mathfrak{s u}(2)$, para isto, seja $X \in \mathfrak{s u}(2)$, determinemos como deve ser as entradas de $X$. Como $X \in \mathfrak{s u}(2), \log$ existem $a, b, c, d, e, f, g, h \in \mathbb{R}$ tais que

$$
X=\left(\begin{array}{cc}
a+i b & c+i d \\
e+i f & g+i h
\end{array}\right)
$$

uma vez que $\operatorname{tr} X=0$, devemos ter $(a+g)+i(b+h)=0 \operatorname{logo} a=-g$ e $b=-h$. E ainda, $X$ satisfaz $X+X^{*}=0$, daí

$$
\begin{aligned}
& \left(\begin{array}{cc}
a+i b & c+i d \\
e+i f & g+i h
\end{array}\right)+\left(\begin{array}{cc}
a-i b & e-i f \\
c-i d & g-i h
\end{array}\right)=0 \\
& \Rightarrow\left(\begin{array}{cc}
2 a & c+e+i(d-f) \\
e+c+i(f-d) & 2 g
\end{array}\right)=0
\end{aligned}
$$

dessa forma $a=g=0, c=-e$ e $d=f$. Portanto se $X \in \mathfrak{s u}(2)$ então $X$ é da 
forma

$$
X=\left(\begin{array}{cc}
i b & c+i d \\
-c+i d & -i b
\end{array}\right)
$$

Vamos determinar como deve ser as entradas de $A$ se $A \in S U(2)$, sabemos de (6.2) que $\operatorname{det} A=1$, assim, se

$$
A=\left(\begin{array}{ll}
\alpha & \beta \\
\gamma & \theta
\end{array}\right), \alpha, \beta, \gamma, \theta \in \mathbb{C}
$$

então, $\alpha \theta-\gamma \beta=1$. A matriz $A$ também satisfaz $A \cdot A^{*}=A^{*} \cdot A=I$, isto significa que $A^{-1}=A^{*}$, ou seja

$$
A=\left(\begin{array}{ll}
\bar{\alpha} & \bar{\gamma} \\
\bar{\beta} & \bar{\theta}
\end{array}\right), \alpha, \beta, \gamma, \theta \in \mathbb{C}
$$

mas a matriz inversa de $A$ é dada por

$$
A^{-1}=\frac{1}{\operatorname{det} A}\left(\begin{array}{cc}
\theta & -\beta \\
-\gamma & \alpha
\end{array}\right)=\left(\begin{array}{cc}
\theta & -\beta \\
-\gamma & \alpha
\end{array}\right)
$$

dessa forma, temos que ter

$$
\left(\begin{array}{cc}
\bar{\alpha} & \bar{\gamma} \\
\bar{\beta} & \bar{\theta}
\end{array}\right)=\left(\begin{array}{cc}
\theta & -\beta \\
-\gamma & \alpha
\end{array}\right)
$$

$\operatorname{assim} \theta=\bar{\alpha}$ e $\beta=-\bar{\gamma}$, portanto, se $A \in S U(2)$ então $A$ é da forma

$$
A=\left(\begin{array}{cc}
\alpha & \beta \\
-\bar{\beta} & \bar{\alpha}
\end{array}\right)
$$

A partir de (6.3), vamos explicitar como deve ser as entradas de $X \in$ $\mathfrak{s u}(3)$. Sendo $X \in \mathfrak{s u}(3)$, existem números reais $a_{j}, b_{j} j=1, \ldots, 9$, tais que

$$
X=\left(\begin{array}{ccc}
a_{1}+i b_{1} & a_{2}+i b_{2} & a_{3}+i b_{3} \\
a_{4}+i b_{4} & a_{5}+i b_{5} & a_{6}+i b_{6} \\
a_{7}+i b_{7} & a_{8}+i b_{8} & a_{9}+i b_{9}
\end{array}\right)
$$

sabemos que $X$ satisfaz $X+X^{*}=0$ e $\operatorname{tr} X=0$, assim

$$
X+X^{*}=\left(\begin{array}{ccc}
a_{1}+i b_{1} & a_{2}+i b_{2} & a_{3}+i b_{3} \\
a_{4}+i b_{4} & a_{5}+i b_{5} & a_{6}+i b_{6} \\
a_{7}+i b_{7} & a_{8}+i b_{8} & a_{9}+i b_{9}
\end{array}\right)+\left(\begin{array}{ccc}
a_{1}-i b_{1} & a_{4}-i b_{4} & a_{7}-i b_{7} \\
a_{2}-i b_{2} & a_{5}-i b_{5} & a_{8}-i b_{8} \\
a_{3}-i b_{3} & a_{6}-i b_{6} & a_{9}-i b_{9}
\end{array}\right)
$$


resultando em

$$
\left(\begin{array}{ccc}
2 a_{1} & a_{4}+a_{2}+i\left(b_{2}-b_{4}\right) & a_{3}+a_{7}+i\left(b_{3}-b_{7}\right) \\
a_{4}+a_{2}+i\left(b_{4}-b_{2}\right) & 2 a_{5} & a_{6}+a_{8}+i\left(b_{6}-b_{8}\right) \\
a_{3}+a_{7}+i\left(b_{7}-b_{3}\right) & a_{6}+a_{8}+i\left(b_{8}-b_{6}\right) & 2 a_{9}
\end{array}\right)=0
$$

obtemos

$$
a_{1}=a_{5}=a_{9}=0
$$

e ainda

$$
\begin{aligned}
& a_{4}+a_{2}+i\left(b_{2}-b_{4}\right)=0 \Rightarrow a_{2}=-a_{4} \text { e } b_{2}=b_{4} \\
& a_{3}+a_{7}+i\left(b_{7}-b_{3}\right)=0 \Rightarrow a_{2}=-a_{7} \text { e } b_{7}=b_{3} \\
& a_{6}+a_{8}+i\left(b_{8}-b_{6}\right)=0 \Rightarrow a_{6}=-a_{8} \text { e } b_{8}=b_{6}
\end{aligned}
$$

Agora falta condições a respeito de $b_{1}, b_{5}$ e $b_{9}$. Como o $\operatorname{tr} X=0$, segue que $b_{9}=-b_{1}-b_{5}$. Portanto se $X \in \mathfrak{s u}(3)$, então

$$
X=\left(\begin{array}{ccc}
i b_{1} & a_{2}+i b_{2} & a_{3}+i b_{3} \\
-a_{2}+i b_{2} & i b_{5} & a_{6}+i b_{6} \\
-a_{3}+i b_{3} & -a_{6}+i b_{6} & -i\left(b_{1}+b_{5}\right)
\end{array}\right)
$$

\section{2}

\section{A órbita coadjunta de $S U(n)$ é difeomórfica a uma variedade bandeira}

O objetivo principal desta seção é mostrar que a órbita coadjunta de $S U(n)$ por um elemento $X \in \mathfrak{s u}^{*}(n)$ é difeomórfica a uma determinada variedade bandeira, explicitaremos essas órbitas para os casos em que o grupo é $S U(2)$ e $S U(3)$.

Da Proposição 6.22, temos

$$
\mathfrak{s u}(n)=\left\{X \in M_{n}(\mathbb{C}) \mid X+X^{*}=0_{n} \text { e } \operatorname{tr} X=0\right\}
$$

Se $X \in \mathfrak{s u}(n)$, então $X$ é anti-hermitiana e portanto pode ser diagonalizada com a diagonal sendo os autovalores os quais são imaginários puro, essa diagonalização pode ser efetuada conjugando com uma matriz unitária especial, isto é, existe $g \in S U(n)$ tal que

$$
\operatorname{Ad}_{g} X=g X g^{-1}=i\left(\begin{array}{cccc}
\lambda_{1} & 0 & \cdots & 0 \\
0 & \lambda_{2} & \cdots & 0 \\
\vdots & \vdots & \ddots & \vdots \\
0 & 0 & \cdots & \lambda_{n}
\end{array}\right)
$$


onde $\lambda_{1}, \ldots, \lambda_{n} \in \mathbb{R}, \sum_{j=1}^{n} \lambda_{j}=0$ e $\operatorname{tr}\left(g X g^{-1}\right)=0$, pois $g X g^{-1} \in \mathfrak{s u}(n)$. Duas matrizes diagonais estão na mesma órbita da ação adjunta de $S U(n)$ se, e somente se, os elementos da diagonal de uma das matrizes é uma permutação dos elementos da diagonal da outra.

Note que a órbita adjunta $\mathcal{O}_{X}$ de $S U(n)$ por $X \in \mathfrak{s u}(n)$ é então determinada pela $n$-upla $\left(\lambda_{1}, \ldots, \lambda_{n}\right) \in \mathbb{R}^{n}$, ou seja, em toda órbita tem um ponto da forma $H=i \operatorname{diag}\left(\lambda_{1}, \ldots, \lambda_{n}\right)$, portanto podemos escrever

$$
\mathcal{O}_{X}=\left\{g H g^{-1} \mid g \in S U(n)\right\}
$$

com $\lambda_{1}+\cdots+\lambda_{n}=0$ e $\lambda_{1} \geq \cdots \geq \lambda_{n}$, onde os $\lambda_{j}$ 's já foram reorganizados por uma permutação adequada.

Uma matriz de permutação $n \times n$ é uma matriz obtida permutando as linhas da matriz identidade $n \times n$. Cada linha e coluna, portanto, contém precisamente um único 1 e as demais entradas são 0. Uma matriz de permutação é não singular e seu determinante é sempre \pm 1 . Além disto, essas matrizes satisfazem $A \cdot A^{T}=I$. Dessa forma, faz sentido tomar matrizes de permutação em $S U(n)$, bastando apenas tomar o devido cuidado para que o determinante seja positivo.

É fácil ver que conjugando por uma matriz de permutação adequada em $S U(n)$ podemos agrupar os autovalores que são iguais juntos para que possamos escrever $\operatorname{Ad}_{g} X$ na forma de bloco como a seguir

$$
\operatorname{Ad}_{g} X=i\left(\begin{array}{ccc}
\lambda_{1} I_{d_{1}} & \cdots & 0 \\
\vdots & \ddots & \vdots \\
0 & \cdots & \lambda_{r} I_{d_{r}}
\end{array}\right)=H
$$

onde $I_{d_{j}}$ é a matriz identidade de ordem $d_{j} \times d_{j}$, sendo $d_{j}$ a multiplicidade do autovalor $i \lambda_{j}$, note que $r \leq n, d_{1}+\cdots+d_{r}=n$ e $\lambda_{1} d_{1}+\cdots+\lambda_{r} d_{r}=0$, esta última se deve ao fato de que $\operatorname{Ad}_{g} X \in \mathfrak{s u}(n)$, dessa forma, $\operatorname{Ad}_{g} X$ satisfaz $\operatorname{tr}\left(\operatorname{Ad}_{g} X\right)=\lambda_{1} d_{1}+\cdots+\lambda_{r} d_{r}=0$.

Quanto ao estabilizador, caso o grupo que estivesse sendo estudado fosse $G=U(n)$, então dado $X \in \mathfrak{u}(n)$, a órbita de $U(n)$ por $X$ conteria um elemento $H$, com $H=i \operatorname{diag}\left(\lambda_{1} I_{d_{1}}, \ldots, \lambda_{r} I_{d_{r}}\right)$ nas condições mencionadas acima adaptando para o grupo $U(n)$, o estabilizador de $H$ seria dado pelo subgrupo

$$
E_{d}=\left\{g=\operatorname{diag}\left(a_{1}, \ldots, a_{r}\right) \in U(n) \mid a_{j} \in U\left(d_{j}\right), j=1, \ldots, r\right\}
$$


sendo este subgrupo rotulado pela sequência $d=\left(d_{1}, \ldots, d_{r}\right)$ das multiplicidades dos autovalores de $H$. Vamos verificar esse fato. Seja $g \in U(n)_{H}$, não é difícil ver que $g$ é diagonal por blocos e que os blocos são de tamanhos $d_{1}, \ldots, d_{r}$, assim

$$
g=\operatorname{diag}\left(a_{1}, \ldots, a_{r}\right)
$$

onde o $a_{j}$ é uma matriz quadrada $d_{j} \times d_{j}$. Sendo $g \in U(n)_{H}$ temos que

$$
\operatorname{Ad}_{g} H=H \Rightarrow g H g^{-1}=H
$$

isto significa que o $j$-ésimo bloco de $g$ fixa o $j$-ésimo bloco de $H$, o qual denotaremos por $h_{j}$, assim, temos que ter

$$
a_{j} \cdot h_{j} \cdot a_{j}^{-1}=h_{j}
$$

mas

$$
\begin{aligned}
a_{j} \cdot h_{j} \cdot a_{j}^{-1} & =a_{j} \cdot i \lambda_{j} \cdot I_{d_{j}} \cdot a_{j}^{-1} \\
& =i \lambda_{j} a_{j} \cdot a_{j}^{-1}
\end{aligned}
$$

$\log \mathrm{o} a_{j}^{*}=a_{j}^{-1}$ e portanto $a_{j} \in U\left(d_{j}\right)$, como $g$ e $a_{j}$ foram tomados arbitrariamente, segue que $U(n)_{H}=E_{d}$, como queríamos provar.

Seja $D$ o conjunto de todas as sequências que rotulam o subgrupo $E_{d}$, isto é, sequências $d=\left(d_{1}, \ldots, d_{r}\right)$ de comprimentos $r=1,2, \ldots$ de inteiros positivos satisfazendo $d_{1}+\cdots+d_{r}=n$. Subgrupos $E_{d}$ e $E_{s}$, onde $d, s \in D$, são conjugados em $U(n)$ se, e somente se, as sequências $d$ e $s$ diferirem por uma permutação. Consequentemente, as subvariedades com tipo de órbita $\left(E_{d}\right)$ correspondem bijetivamente a partições $n=d_{1}+\cdots+d_{r}$.

Agora retornando ao caso em que $G=S U(n)$, o estabilizador de

$$
H=i \operatorname{diag}\left(\lambda_{1} I_{d_{1}}, \ldots, \lambda_{r} I_{d_{r}}\right)
$$

é dado por $G_{d}=S U(n) \cap E_{d}$, onde $d=\left(d_{1}, \ldots, d_{r}\right)$, ou seja,

$$
G_{d}=\left\{g \in S U(n) \mid g H g^{-1}=H\right\}
$$

se $g \in G_{d}$, então $g$ é diagonal por blocos, $g=\left(a_{1}, \ldots, a_{r}\right)$ onde o tamanho do $j$-ésimo bloco na diagonal, corresponde a dimensão do $j$-ésimo autoespaço da matriz $H$.

Observação 6.23 A dimensão do autoespaço nem sempre é igual a multiplicidade do autovalor. Temos que 
- A multiplicidade do autovalor é chamada de multiplicidade algébrica.

- A dimensão do autoespaço associado é chamada de multiplicidade geométrica.

Mas neste caso em que a matriz é diagonal, a dimensão do autoespaço coincide com a multiplicidade do autovalor. Agora se todos os autovalores são distintos, teremos que o subgrupo de isotropia $G_{d} \simeq \mathbb{T}^{n-1}$ é o toro $(n-1)$-dimensional.

Suponha que um autovalor de $X \in \mathfrak{s u}(n)$ tenha multiplicidade $n-1$, ou seja, $X$ tem apenas dois autovalores distintos, garantindo assim que na órbita adjunta de $S U(n)$ por $X$ existe o ponto $H$ dado por

$$
H=i \operatorname{diag}(\underbrace{\lambda_{1}, \ldots, \lambda_{1}}_{n-1 \text { vezes }}, \lambda_{2})
$$

neste caso o estabilizador de $H$ é o subgrupo de $S U(n)_{H}$ o qual é composto pelas matrizes da forma

$$
g=\left(\begin{array}{cc}
a & 0_{n-1 \times 1} \\
0_{1 \times n-1} & f
\end{array}\right), a \in U(n-1), f \in \mathbb{C}
$$

tal que $\operatorname{det} g=1, \operatorname{assim} \operatorname{det}(a) \cdot f=1$, uma vez que o determinante de uma matriz unitária é um número complexo com norma igual um, segue que $f=\overline{\operatorname{det}(a)}$. E dessa forma, a órbita de $S U(n)$ por $H$ pode ser realizada por

$$
S U(n) / U(n-1)=U(n) /(U(1) \times U(n-1))=G r_{\mathbb{C}}(1, n)=\mathbb{C P}^{n-1} .
$$

O quociente acima é a variedade bandeira complexa $\mathbb{F}_{\mathbb{C}}(1, n-1)$, isto é o que será abordado a seguir, mais precisamente apresentamos um resultado que diz que a a órbita adjunta de $S U(n)$ por $H=i \operatorname{diag}\left(\lambda_{1} I_{d_{1}}, \ldots, \lambda_{r} I_{d_{r}}\right)$ é a variedade bandeira complexa $\mathbb{F}_{\mathbb{C}}\left(d_{1}, \ldots, d_{r}\right)$.

\subsection{1}

\section{Variedade Bandeira}

Afim de compreendermos melhor a respeito das variedades bandeira, extraímos de [19] o seguinte resumo.

Considere os inteiros $0<k_{1}<\cdots<k_{r-1}<n$. Uma bandeira de tipo $\left(k_{1}, \cdots, k_{r-1}\right)$ em $\mathbb{K}^{n}$, onde $\mathbb{K}=\mathbb{R}$ ou $\mathbb{K}=\mathbb{C}$, é uma sequência ascendente de subespaços vetoriais $V_{1} \subset \cdots \subset V_{r-1} \subset \mathbb{K}^{n}$ onde $\operatorname{dim}\left(V_{i}\right)=k_{i}$. Definindo $W_{1}=V_{1}$ e $W_{i+1}$ o complemento ortogonal de $V_{i}$ em $V_{i+1}$, ou seja, $V_{i+1}=V_{i} \oplus W_{i+1}$, com cada bandeira pode-se associar uma decomposição $\mathbb{K}^{n}=W_{1} \oplus \cdots \oplus W_{r}$ em subespaços mutuamente ortogonais, e isto define uma 
bijeção do conjunto das bandeiras de tipo $\left(k_{1}, \ldots, k_{r-1}\right)$ com o conjunto das decomposições de somas diretas ortogonais de $\mathbb{K}^{n}$ em subespaços de dimensões $\left(n_{1}, \ldots, n_{r}\right)$, onde

$$
n_{1}=k_{1}, n_{r}=n-k_{r-1} \text { e } n_{i+1}=k_{i+1}-k_{i}
$$

denote qualquer um desses conjuntos por $\mathbb{F}_{\mathbb{K}}\left(n_{1}, \ldots, n_{r}\right)$, é possível mostrar que

$$
\begin{aligned}
& \mathbb{F}_{\mathbb{R}}\left(n_{1}, \ldots, n_{r}\right)=O(n) /\left(O\left(n_{1}\right) \times \cdots \times O\left(n_{r}\right)\right) \\
& \mathbb{F}_{\mathbb{C}}\left(n_{1}, \ldots, n_{r}\right)=U(n) /\left(U\left(n_{1}\right) \times \cdots \times U\left(n_{r}\right)\right)
\end{aligned}
$$

onde os subgrupos consistem em matrizes diagonais por blocos

$$
\left(\begin{array}{cccc}
a_{1} & 0 & \ldots & 0 \\
0 & a_{2} & \ddots & \vdots \\
\vdots & \ddots & \ddots & 0 \\
0 & \ldots & 0 & a_{r}
\end{array}\right)
$$

onde o bloco $a_{i}$ é um elemento de $O\left(n_{i}\right)$ ou $U\left(n_{i}\right)$, respectivamente, $i=$ $1, \ldots, r$. Estas igualdades são usadas para definir uma estrutura suave em $\mathbb{F}_{\mathbb{K}}\left(n_{1}, \ldots, n_{r}\right)$. Note que temos $\mathbb{F}_{\mathbb{K}}(k, n-k)=G r_{\mathbb{K}}(k, n)$, dessa forma $\mathbb{F}_{\mathbb{C}}(1, n-1)=G r_{\mathbb{C}}(1, n)=\mathbb{C P}^{n-1}$, pois a Grassmaniana é dada por

$$
\begin{aligned}
& G r_{\mathbb{R}}(k, n)=O(n) /(O(k) \times O(n-k)) \\
& G r_{\mathbb{C}}(k, n)=U(n) /(U(k) \times U(n-k))
\end{aligned}
$$

O resultado a seguir foi extraído de [27].

Proposição 6.24 A órbita adjunta $\mathcal{O}_{H}$ onde $H$ é da forma

$$
H=\operatorname{Ad}_{g} X=i \operatorname{diag}\left(\lambda_{1} I_{d_{1}}, \ldots, \lambda_{r} I_{d_{r}}\right)
$$

com $X \in \mathfrak{s u}(n)$ e $g \in S U(n)$, é difeomórfica a variedade bandeira complexa $\mathbb{F}_{\mathbb{C}}\left(d_{1}, \ldots, d_{r}\right)$ via um difeomorfismo $S U(n)$-equivariante.

Proof. Para qualquer $g \in S U(n)$ defina a seguinte bandeira

$$
\varphi\left(\operatorname{Ad}_{g} H\right) \in \mathbb{F}_{\mathbb{C}}\left(d_{1}, \ldots, d_{r}\right)
$$

onde

$$
\varphi\left(\operatorname{Ad}_{g} H\right)=0 \subset g \cdot V_{1} \subset g \cdot V_{1} \oplus g \cdot V_{2} \subset \cdots \subset \bigoplus_{j=1}^{r} g \cdot V_{j}=\mathbb{C}^{n}
$$


onde $V_{j}$ é o autoespaço associado ao autovalor $i \lambda_{j}$, note que $\operatorname{dim} V_{j}=d_{j}$ pois $H$ é uma matriz diagonal. A matriz $\operatorname{Ad}_{g} H$ tem autovalores $i \lambda_{1}, \ldots, i \lambda_{r}$, de fato, uma vez que os $i \lambda_{j}^{\prime} s$ são raízes da equação

$$
\operatorname{det}(H-x I)=0
$$

e temos que

$$
\begin{array}{rlr}
\operatorname{det}\left(\operatorname{Ad}_{g} H-x I\right) & =\operatorname{det}\left(g H g^{-1}-x g g^{-1}\right) & \\
& =\operatorname{det} g \cdot \operatorname{det}(H-x I) \cdot \operatorname{det} g^{-1} & \\
& =\operatorname{det}(H-x I) & \text { pois } \operatorname{det} g=1
\end{array}
$$

portanto $i \lambda_{1}, \ldots, i \lambda_{r}$ são autovalores de $\operatorname{Ad}_{g} H$. Temos assim que os autoespaços correspondentes são $V_{1}, \ldots, V_{r}$. Desde que os autovalores e autoespaços determinam unicamente uma matriz segue que $\varphi$ é uma aplicação injetiva bem definida

$$
\varphi: \mathcal{O}_{H} \rightarrow \mathbb{F}_{\mathbb{C}}\left(d_{1}, \ldots, d_{r}\right)
$$

$\varphi$ é equivariante, pois

$$
\varphi\left(\operatorname{Ad}_{h}\left(\operatorname{Ad}_{g} H\right)\right)=\varphi\left(\operatorname{Ad}_{h g} H\right)=0 \subset h g \cdot V_{1} \subset \cdots \subset \bigoplus_{j=1}^{r} h g \cdot V_{j}=\mathbb{C}^{n} .
$$

Desde que $S U(n)$ age transitivamente em frames unitários orientados em $\mathbb{C}^{n}$ logo também age transitivamente em bandeiras, portanto $\varphi$ é uma bijeção suave $S U(n)$-equivariante.

Sabendo que a órbita adjunta de $S U(n)$ por $X \in \mathfrak{s u}(n)$ é dada por uma variedade bandeira adequada, os exemplos a seguir explicitam as órbitas de $S U(2)$ e $S U(3)$.

Exemplo 6.25 Considere o grupo $S U(2)$, dado $X \in \mathfrak{s u}(2)$, sabemos que existe $g \in S U(2)$ tal que $\operatorname{Ad}_{g} X=H=\operatorname{diag}(i \lambda,-i \lambda)$, e a órbita de $S U(2)$ passando por $H$ é dada por

$$
\mathcal{O}_{H}=\left\{g \cdot \operatorname{diag}(i \lambda,-i \lambda) \cdot g^{-1} \mid g \in S U(2)\right\}
$$

a qual pode ser de dois tipos, se $\lambda \neq 0$ o estabilizador consiste das matrizes diagonais $g=\operatorname{diag}(\alpha, \bar{\alpha})$ com $\alpha \in U(1)$, de fato, se $g \in S U(2)$, então de (6.4) temos que

$$
g=\left(\begin{array}{cc}
\alpha & \beta \\
-\bar{\beta} & \bar{\alpha}
\end{array}\right)
$$


assim $g \in S U(2)_{H}$ se

$$
\begin{aligned}
\left(\begin{array}{cc}
\alpha & \beta \\
-\bar{\beta} & \bar{\alpha}
\end{array}\right) \cdot\left(\begin{array}{cc}
i \lambda & 0 \\
0 & -i \lambda
\end{array}\right) \cdot\left(\begin{array}{cc}
\bar{\alpha} & -\beta \\
\bar{\beta} & \alpha
\end{array}\right) & =\left(\begin{array}{cc}
i \lambda & 0 \\
0 & -i \lambda
\end{array}\right) \\
\left(\begin{array}{cc}
i \lambda\left(|\alpha|^{2}-|\beta|^{2}\right) & -2 i \lambda \alpha \beta \\
-2 i \lambda \alpha \beta & i \lambda\left(|\beta|^{2}-|\alpha|^{2}\right)
\end{array}\right) & =\left(\begin{array}{cc}
i z_{j} \\
i z_{j} & 0
\end{array}\right)
\end{aligned}
$$

assim

$$
\left\{\begin{aligned}
|\alpha|^{2}-|\beta|^{2} & =1 \\
\alpha \beta & =0
\end{aligned} \Rightarrow \alpha \in U(1) \text { e } \beta=0\right.
$$

portanto,

$$
S U(2)_{H}=\{g \in S U(2) \mid g=\operatorname{diag}(\alpha, \bar{\alpha}) \quad \alpha \in U(1)\}
$$

consequentemente, a órbita de $S U(2)$ por $H$ pode ser realizada por

$$
S U(2) / U(1) \equiv U(2) /(U(1) \times U(1))=\mathbb{F}_{\mathbb{C}}(1,1)=G r_{\mathbb{C}}(1,2)=\mathbb{C P}^{1} \simeq \mathbb{S}^{2}
$$

se $\lambda=0$, neste caso o estabilizador é $S U(2)$ e a órbita consiste apenas da matriz nula, ou seja, um ponto em $\mathfrak{s u}(2)$, se pensarmos na bijeção com $\mathbb{R}^{3}$, temos que esse ponto é a origem e as demais órbitas, $\lambda \neq 0$, são esferas concêntricas com centro na origem e raio igual ao módulo do autovalor da matriz X tomada inicialmente.

Exemplo 6.26 Considere o grupo $S U(3)$, dado $X \in \mathfrak{s u}(3)$, com autovalores $i \lambda_{1}$, i $\lambda_{2}$ e $i \lambda_{3}$, sujeitos a $\lambda_{1}+\lambda_{2}+\lambda_{3}=0$. Sabemos que existe $g \in S U(3)$ tal que $\operatorname{Ad}_{g} X=H=\operatorname{diag}\left(i \lambda_{1}, i \lambda_{2}, i \lambda_{3}\right)$, portanto a órbita adjunta de $S U(3)$ por $H$ é dada por

$$
\mathcal{O}_{H}=\left\{g \cdot \operatorname{diag}\left(i \lambda_{1}, i \lambda_{2}, i \lambda_{3}\right) \cdot g^{-1} \mid g \in S U(3)\right\}
$$

note que temos três possibilidades para os autovalores de $X$, o que acarreta em três tipos de órbitas. As possibilidades para os autovalores são:

1. São dois a dois distintos.

2. Dois iguais e um distinto.

3. Os três iguais.

Suponha que os autovalores $i \lambda_{1}$, $i \lambda_{2}$ e $i \lambda_{3}$ são dois a dois distintos, para este caso o estabilizador consiste de matrizes diagonais $g=\operatorname{diag}(\alpha, \beta, \gamma) \in S U(3)$, 
os quais satisfazem

$$
\begin{aligned}
g \cdot H \cdot g^{-1} & =H \\
\operatorname{diag}(\alpha, \beta, \gamma) \cdot \operatorname{diag}\left(i \lambda_{1}, i \lambda_{2}, i \lambda_{3}\right) \cdot \operatorname{diag}(\bar{\alpha}, \bar{\beta}, \bar{\gamma}) & =\operatorname{diag}\left(i \lambda_{1}, i \lambda_{2}, i \lambda_{3}\right) \\
\operatorname{diag}\left(i \lambda_{1}|\alpha|^{2}, i \lambda_{2}|\beta|^{2}, i \lambda_{3}|\gamma|^{2}\right) & =\operatorname{diag}\left(i \lambda_{1}, i \lambda_{2}, i \lambda_{3}\right)
\end{aligned}
$$

como $g \in S U(3)_{H}$ segue que

$$
\left\{\begin{array}{rl}
\alpha \beta \gamma & =1 \\
|\alpha|^{2},|\beta|^{2},|\gamma|^{2} & =1
\end{array} \Rightarrow \gamma=\overline{\alpha \beta}\right.
$$

portanto,

$$
g=\operatorname{diag}(\alpha, \beta, \overline{\alpha \beta}), \text { onde } \alpha, \beta \in U(1)
$$

dessa forma, a órbita coadjunta pode ser realizada por

$$
S U(3) / \mathbb{T}^{2}=S U(3) /(U(1) \times U(1))=U(3) / U(1)^{3}=\mathbb{F}_{\mathbb{C}}(1,1,1)
$$

a qual é a variedade bandeira de dimensão 6, pois $U(n)$ é um subgrupo de Lie propriamente mergulhado em $G L(n, \mathbb{C})$ de dimensão $n^{2}$.

Se os autovalores são tais que $\lambda_{1}=\lambda_{2} \neq \lambda_{3}$, para este caso o estabilizador consiste de matrizes da forma

$$
g=\left(\begin{array}{lll}
a & b & 0 \\
c & d & 0 \\
0 & 0 & f
\end{array}\right) \in S U(3)
$$

analisemos as condições sobre as entradas de $g$. Desde que $g \in S U(3), g$ satisfaz $g^{*} \cdot g=g \cdot g^{*}=I$, dessa forma

$$
\left(\begin{array}{ccc}
a & b & 0 \\
c & d & 0 \\
0 & 0 & f
\end{array}\right) \cdot\left(\begin{array}{ccc}
\bar{a} & \bar{c} & 0 \\
\bar{b} & \bar{d} & 0 \\
0 & 0 & \bar{f}
\end{array}\right)=\left(\begin{array}{ccc}
|a|^{2}+|b|^{2} & a \bar{c}+b \bar{d} & 0 \\
c \bar{a}+d \bar{b} & |c|^{2}+|d|^{2} & 0 \\
0 & 0 & |f|^{2}
\end{array}\right)=I_{3}
$$

isto nos fornece que $|a|^{2}+|b|^{2}=1,|c|^{2}+|d|^{2}=1, a \bar{c}+b \bar{d}=0$

$$
\left(\begin{array}{ll}
a & b \\
c & d
\end{array}\right) \in U(2) e|f|^{2}=1
$$

e ainda, como $\operatorname{det} g=1 \Rightarrow(a d-c b) \cdot f=1$, usando esta última informação e 
o fato que $|f|^{2}=1$, concluímos que

$$
g=\left(\begin{array}{ccc}
a & b & 0 \\
c & d & 0 \\
0 & 0 & \frac{a d-b c}{a d}
\end{array}\right) \in S U(3)
$$

portanto, a órbita coadjunta pode ser realizada por

$$
S U(3) / U(2)=U(3) /(U(1) \times U(2))=\mathbb{F}_{\mathbb{C}}(1,2)=G r_{\mathbb{C}}(1,3) \simeq \mathbb{C P}^{2}
$$

órbitas deste tipo tem dimensão 4. Este caso é equivalente a qualquer outra permutação possível onde tenha autovalores com multiplicidades 1 e 2 .

Finalmente, se os autovalores são tais que $\lambda_{1}=\lambda_{2}=\lambda_{3}$, como os autovalores estão sujeitos a $\lambda_{1}+\lambda_{2}+\lambda_{3}=0$, segue que $\lambda_{i}=0$, neste caso o estabilizador é $S U(3)$ e a órbita é apenas a matriz nula, fazendo a bijeção com $\mathbb{R}^{8}$, a órbita será apenas a origem.

Uma vez que as ações adjunta e coadjunta são equivalentes para $S U(n)$, veja a Observação 8.19, e que as folhas simpléticas coincidem com as órbitas coadjuntas, concluímos que as folhas simpléticas de $\mathfrak{s u} *(3)$ têm dimensões 0,4 e 6, enquanto que para $\mathfrak{s u}^{*}(2)$ as folhas simpléticas têm dimensão 0 e 2.

\section{3}

\section{Base da álgebra de Lie de $\mathfrak{s u}(n)$}

A álgebra de Lie $\mathfrak{s u}(n)$ do grupo $S U(n)$ tem dimensão $n^{2}-1$ e sua base é dada pelos elementos $e_{j}=-\frac{i}{2} E_{j}$ onde os $E_{j}$ 's que geram as entradas fora da diagonal são um total de $n(n-1)$ elementos e são da forma

$$
\left(\begin{array}{llll}
0 & 1 & & 0 \\
1 & 0 & & \\
& & \ddots & \\
& & & \\
0 & & & 0
\end{array}\right),\left(\begin{array}{ccccc}
0 & 0 & 1 & & 0 \\
0 & 0 & & & \\
1 & & 0 & & \\
& & & \ddots & \\
0 & & & & 0
\end{array}\right) \cdots\left(\begin{array}{ccccc}
0 & 0 & & & \\
0 & 0 & & & \\
& & \ddots & & \\
& & & 0 & 1 \\
& & & 1 & 0
\end{array}\right),
$$

$$
\left(\begin{array}{cccc}
0 & -i & & 0 \\
i & 0 & & \\
& & \ddots & \\
& & & \\
0 & & & 0
\end{array}\right),\left(\begin{array}{ccccc}
0 & 0 & -i & & 0 \\
0 & 0 & & & \\
i & & 0 & & \\
& & & \ddots & \\
0 & & & & 0
\end{array}\right) \cdots\left(\begin{array}{ccccc}
0 & 0 & & & \\
0 & 0 & & & \\
& & \ddots & & \\
& & & 0 & -i \\
& & & & \\
& & & &
\end{array}\right)
$$


e os $n-1$ elementos da base que geram a diagonal são os $E_{j}$ 's da forma

$$
\begin{gathered}
E_{1}=\operatorname{diag}(1,-1,0, \ldots, 0), \\
E_{2}=\frac{1}{\sqrt{3}} \operatorname{diag}(1,1,-2, \ldots, 0), \\
E_{3}=\frac{1}{\sqrt{6}} \operatorname{diag}(1,1,1,-3, \ldots, 0) \\
\vdots \\
E_{n-1}=\sqrt{\frac{2}{n(n-1)}} \operatorname{diag}(1,1, \ldots, 1,-n+1)
\end{gathered}
$$

Como $\operatorname{dim} \mathfrak{s u}(n)=n^{2}-1$, assim, existe uma bijeção entre $X \in \mathfrak{s u}(n)$ e $v_{X} \in \mathbb{R}^{n^{2}-1}$, onde as $(n-1)$ primeiras entradas de $v_{X}$ são formada pelas partes imaginárias da diagonal de $X$ e as últimas $n(n-1)$ entradas restantes de $v_{X}$ são formadas pela parte real e imaginária de cada entrada acima da diagonal de $X$, ou seja, se

$$
X=\left(\begin{array}{cccc}
i x_{11} & & & \\
& \ddots & x_{k j} & \\
& & \ddots & \\
& & & i x_{n n}
\end{array}\right), x_{k j}=a_{k j}+i b_{k j}
$$

o vetor $v_{X}$ é da forma

$$
v_{X}=\underbrace{\left(a_{11}, \ldots, a_{n-1 n-1}\right.}_{n-1}, \underbrace{a_{12}, \ldots, a_{n-1 n}}_{\frac{n(n-1)}{2}}, \underbrace{\left.b_{12}, \ldots, b_{n-1 n}\right)}_{\frac{n(n-1)}{2}}
$$

No que segue faremos mais uma vez uso da equivalência entre as ações adjunta e coadjunta, faremos ainda uma investigação a respeito da ação coadjunta de $S U(n)$ ser ou não livre. Para isto, seja $X \in \mathfrak{s u}(n)$, é conhecido que sempre existe $g \in S U(n)$ tal que

$$
\operatorname{Ad}_{g} X=H=i \operatorname{diag}\left(\lambda_{1} I_{d_{1}}, \ldots, \lambda_{r} I_{d_{r}}\right)
$$

ou seja, $H$ é da forma diagonal, logo $H$ pode ser escrito como combinação linear dos $(n-1)$ elementos da base de $\mathfrak{s u}(n)$ que geram os elementos diagonais. Para sabermos se a ação é livre em $X$, basta investigar se é livre em $H$. Faremos agora uma análise nos $(n-1)$ elementos da base de $\mathfrak{s u}(n)$ os quais geram a diagonal de um elemento genérico de $\mathfrak{s u}(n)$, mais precisamente, determinaremos os estabilizadores desses elementos. Os $(n-1)$ elementos são

$$
e_{j}=\frac{i}{2} E_{j} j=1, \ldots, n-1
$$


onde os $E_{j}$ 's estão listados em (6.8).

Exibiremos os estabilizadores de $e_{1}, e_{2}$ e $e_{n}$, os demais seguem raciocínio análogo.

1. Estabilizador de $e_{1}$. Sendo $e_{1}=\frac{i}{2} \operatorname{diag}(1,-1,0, \ldots, 0)$, precisamos determinar $g \in S U(n)$ de tal forma que $\operatorname{Ad}_{g}^{*} e_{1}=g \cdot e_{1} \cdot g^{*}=e_{1}, \log g$ é da forma

$$
g=\left(\begin{array}{lll}
a & & \\
& b & \\
& & A
\end{array}\right), a, b \in \mathbb{C} \text { e } A \in M(n-2, \mathbb{C})
$$

desde que $g \in S U(n)$, temos que ter $g \cdot g^{*}=g^{*} \cdot g=I$, assim

$$
g \cdot g^{*}=\left(\begin{array}{lll}
a & & \\
& b & \\
& & A
\end{array}\right) \cdot\left(\begin{array}{lll}
\bar{a} & & \\
& \bar{b} & \\
& & A^{*}
\end{array}\right)=\left(\begin{array}{lll}
|a|^{2} & & \\
& |b|^{2} & \\
& & A \cdot A^{*}
\end{array}\right)
$$

analogamente faz-se para $g^{*} \cdot g$ e a conclusão é a mesma, assim, temos que $a, b \in U(1)$ e $A \in U(n-2)$, agora levando em consideração a condição de que $\operatorname{det} g=1$, pois $g \in S U(n)$, segue que $\operatorname{det} A=\overline{a b}$. Portanto,

$S U(n)_{e_{1}}=\{g=\operatorname{diag}(a, b, A) \mid a, b \in U(1), A \in U(n-2) \mathrm{e} \operatorname{det} A=\overline{a b}\}$

2. Estabilizador de $e_{2}$. Sendo $e_{2}=\frac{i}{2 \sqrt{3}} \operatorname{diag}(1,1,-2,0, \ldots, 0)$, precisamos determinar $g \in S U(n)$ de tal forma que $\operatorname{Ad}_{g}^{*} e_{2}=g \cdot e_{2} \cdot g^{*}=e_{2}$, logo $g$ é da forma

$$
g=\left(\begin{array}{lll}
A & & \\
& b & \\
& & C
\end{array}\right), A \in M(2, \mathbb{C}), b \in \mathbb{C} \text { e } C \in M(n-3, \mathbb{C})
$$

desde que $g \in S U(n)$, temos que ter $g \cdot g^{*}=g^{*} \cdot g=I$, assim

$$
g \cdot g^{*}=\left(\begin{array}{ccc}
A & & \\
& b & \\
& & C
\end{array}\right) \cdot\left(\begin{array}{ccc}
A^{*} & & \\
& \bar{b} & \\
& & C^{*}
\end{array}\right)=\left(\begin{array}{ccc}
A \cdot A^{*} & & \\
& |b|^{2} & \\
& & C \cdot C^{*}
\end{array}\right)
$$

assim, temos que $A \in U(2), b \in U(1)$ e $C \in U(n-3)$, agora levando em consideração a condição de que $\operatorname{det} g=1$, pois $g \in S U(n)$, segue que 
$\operatorname{det} A \cdot b \cdot \operatorname{det} C=1$, equivalentemente $\operatorname{det} A \cdot \operatorname{det} C=\bar{b}$. Portanto,

$$
\begin{aligned}
S U(n)_{e_{2}}= & \{g=\operatorname{diag}(A, b, C) \mid A \in U(2), b \in U(1), C \in U(n-3) \\
& \mathrm{e} \operatorname{det} A \cdot \operatorname{det} C=\bar{b}\}
\end{aligned}
$$

3. Estabilizador de $e_{j}$ para $2<j<n-1$. Existe um padrão para estes estabilizadores e descrevemos isso a seguir. Afim de não carregar a notação, escrevemos apenas $e_{j}=m \operatorname{diag}(\underbrace{1, \ldots, 1}_{j},-j, 0, \ldots, 0)$, onde $m \in \mathbb{C}$, precisamos determinar $g \in S U(n)$ de tal forma que

$$
\operatorname{Ad}_{g}^{*} e_{j}=g \cdot e_{j} \cdot g^{*}=e_{j}
$$

note que $g$ é da forma

$$
g=\left(\begin{array}{lll}
A & & \\
& b & \\
& & C
\end{array}\right), A \in M(j, \mathbb{C}), b \in \mathbb{C} \text { e } C \in M(n-j-1, \mathbb{C})
$$

desde que $g \in S U(n)$, temos que ter $g \cdot g^{*}=g^{*} \cdot g=I$, assim

$$
g \cdot g^{*}=\left(\begin{array}{ccc}
A & & \\
& b & \\
& & C
\end{array}\right) \cdot\left(\begin{array}{ccc}
A^{*} & & \\
& \bar{b} & \\
& & C^{*}
\end{array}\right)=\left(\begin{array}{ccc}
A \cdot A^{*} & & \\
& |b|^{2} & \\
& & C \cdot C^{*}
\end{array}\right)
$$

logo, temos que $A \in U(j), b \in U(1)$ e $C \in U(n-j-1)$, agora levando em consideração a condição de que $\operatorname{det} g=1$, pois $g \in S U(n)$, segue que $\operatorname{det} A \cdot b \cdot \operatorname{det} C=1$, equivalentemente $\operatorname{det} A \cdot \operatorname{det} C=\bar{b}$. Portanto,

$$
\begin{aligned}
S U(n)_{e_{j}}= & \{g=\operatorname{diag}(A, b, C) \mid A \in U(j), b \in U(1), C \in U(n-j-1) \\
& \mathrm{e} \operatorname{det} A \cdot \operatorname{det} C=\bar{b}\}
\end{aligned}
$$

4. Estabilizador de $e_{n-1}$. Sendo $e_{n-1}=\frac{i}{2} \sqrt{\frac{2}{n(n-1)}} \operatorname{diag}(1, \ldots, 1,-n+1)$, precisamos determinar $g \in S U(n)$ de tal forma que

$$
\operatorname{Ad}_{g}^{*} e_{n-1}=g \cdot e_{n-1} \cdot g^{*}=e_{n-1}
$$

$\log g$ é da forma

$$
g=\left(\begin{array}{ll}
A & \\
& b
\end{array}\right), A \in M(n-1, \mathbb{C}), \text { e } b \in \mathbb{C}
$$


desde que $g \in S U(n)$, temos que ter $g \cdot g^{*}=g^{*} \cdot g=I$, assim

$$
g \cdot g^{*}=\left(\begin{array}{cc}
A & \\
& b
\end{array}\right) \cdot\left(\begin{array}{cc}
A^{*} & \\
& \bar{b}
\end{array}\right)=\left(\begin{array}{cc}
A \cdot A^{*} & \\
& |b|^{2}
\end{array}\right)
$$

assim, temos que $A \in U(n-1)$ e $b \in U(1)$, agora levando em consideração a condição de que $\operatorname{det} g=1$, pois $g \in S U(n)$, segue que $\operatorname{det} A=\bar{b}$. Portanto,

$$
S U(n)_{e_{n-1}}=\{g=\operatorname{diag}(A, b) \mid A \in U(n-1), b \in U(1) \text { e } \operatorname{det} A=\bar{b}\} .
$$

Como já foi analisado para o caso de $S U(2)$, pensaremos em $S U(n)$ para $n>2$. Note que para qualquer $j=1, \ldots, n-1$, a ação de $S U(n)$ não é livre em $e_{j}$, de fato apresentamos os subgrupos de isotropia para esses elementos, além disso, note que para $g \in S U(n)$, com $g=\operatorname{diag}(1, \ldots,-1,-1 \ldots, 1), g$ estabiliza $e_{j}$ e ainda mais, qualquer $g$ da forma diagonal com entradas 1 e -1 também estabiliza, desde que a quantidade de entradas -1 seja par, portanto o estabilizador de $e_{j}$ nunca é trivial. Portanto a ação de $S U(n)$ em elementos de $\mathfrak{s u}(n)$ que são diagonais não é livre, e desde que para qualquer $Y \in \mathfrak{s u}(n)$ sempre existe um elementos diagonal na órbita de $S U(n)$ por $Y$, segue que a ação em $Y$ também não é livre.

Faremos agora uma pequena análise afim de mostrar que o toro maximal $\mathbb{T}$ no grupo $S U(n)$ estabiliza os elementos da base de $\mathfrak{s u}(n)$.

Seja $\mathbb{K}$ um grupo de Lie de matrizes compacto e conexo.

Definição 6.27 Um grupo de Lie de matrizes $\mathbb{T}$ é um toro se $\mathbb{T}$ é isomorfo ao produto direto de $k$ cópias do grupo $\mathbb{S}^{1} \backsim U(1)$, para algum $k$.

Exemplo 6.28 Considere o grupo $\mathbb{T}$ de matrizes diagonais $n \times n$ com determinante igual a 1 , todo elemento $g$ de $\mathbb{T}$ pode ser escrito de maneira única como

$$
g=\operatorname{diag}\left(a_{1}, \ldots, a_{n-1},\left(a_{1} \cdot \ldots \cdot a_{n-1}\right)^{-1}\right)
$$

para alguns números complexos $a_{1}, \ldots, a_{n-1}$, com valores absolutos iguais a 1 . Assim $\mathbb{T}$ é isomorfo a $n-1$ cópias de $\mathbb{S}^{1}$

Definição 6.29 Um subgrupo $\mathbb{T}$ de $\mathbb{K}$ é um toro maximal se é um toro e não está propriamente contido em nenhum outro toro em $\mathbb{K}$.

Exemplo 6.30 Considere o caso particular em que $\mathbb{K}=S U(n)$ o toro maximal neste grupo é o toro de dimensão $n-1$ dado por

$$
\mathbb{T}=\left\{g=\operatorname{diag}\left(e^{i \theta_{1}}, \ldots, e^{i \theta_{n-1}}, e^{-i\left(\theta_{1}+\cdots+\theta_{n-1}\right)}\right) ; \theta_{j} \in \mathbb{R}\right\}
$$


A ação adjunta de $S U(n)$ nos elementos da base de $\mathfrak{s u}(n)$ não é livre, de fato, para os elementos $e_{j}$ 's tais que existem duas entradas de $e_{j}$ não nulas, ou seja, $x_{k j}=x_{j k} \neq 0$ para $j>k$, estes elementos estão listados em (6.6), ou $x_{k j}=-x_{j k} \neq 0$ para $j>k$, estes elementos estão listados em (6.7), para $\operatorname{algum} j$ e algum $k$, estes $e_{j}$ 's podem ser estabilizados por

$$
S U(n)_{e_{j}}=\mathbb{T}_{j}=\left\{g=\operatorname{diag}\left(e^{i \theta_{1}}, \ldots, e^{i \theta_{n-1}}, e^{-i\left(\theta_{1}+\cdots+\theta_{n-1}\right)}\right) ; \theta_{k}=\theta_{j}, \theta_{l} \in \mathbb{R}\right\}
$$

enquanto que para os elementos $e_{j}$ 's que são diagonais, ou seja, $x_{k j}=0$ para todo $k \neq j$, estes elementos estão listados em (6.8), podem ser estabilizados por

$$
\mathbb{T}=\left\{g=\operatorname{diag}\left(e^{i \theta_{1}}, \ldots, e^{i \theta_{n-1}}, e^{-i\left(\theta_{1}+\cdots+\theta_{n-1}\right)}\right) ; \theta_{j} \in \mathbb{R}\right\} .
$$

Portanto a ação adjunta de $S U(n)$ não é livre nesses elementos.

\section{4}

$S U(n)$ agindo em $\prod_{j=1}^{m} \mathfrak{s u}(n)$

Nesta seção iniciamos o estudo da ação de $S U(n)$ na variedade $M=$ $\prod_{j=1}^{m} \mathfrak{s u}^{*}(n)$, explicitamos a órbita coadjunta diagonal de $S U(n)$ por um elemento $X$ da variedade, a partir da restrição da ação a essa órbita, obtemos o mapa momento associado $\mu$ o que nos possibilita a obtenção do espaço de polígonos, o qual é realizado pelo quociente $\mu^{-1}(0) / S U(n)$.

Dado $X \in \mathfrak{s u}^{*}(n)$ existe $g \in S U(n)$ tal que

$$
H=\operatorname{Ad}_{g} X=i \operatorname{diag}\left(\lambda_{1} I_{d_{1}}, \ldots, \lambda_{r} I_{d_{r}}\right)
$$

sabemos da Proposição 6.24 que a órbita adjunta de $S U(n)$ por $H$ é difeomórfica a variedade bandeira complexa $\mathbb{F}_{\mathbb{C}}\left(d_{1}, \ldots, d_{r}\right)$, onde $d_{i}$ é a multiplicidade do autovalor $\lambda_{i}$ de $H$. Se considerarmos a ação coadjunta diagonal de $S U(n)$ em $M$, a qual de acordo com a Proposição 4.19 é uma ação de Poisson, a órbita por $X=\left(X_{1}, \ldots, X_{m}\right) \in M$ é dada por

$$
\mathcal{O}_{X}=\mathcal{D}_{X}\left(\prod_{j=1}^{m} \mathbb{F}_{\mathbb{C}}^{j}\left(d_{1}^{j}, \ldots, d_{r_{j}}^{j}\right)\right),
$$

onde $d_{l}^{j}$ é a multiplicidade do autovalor $i \lambda_{l}^{j}$ de $X_{j}$, deixando claro que $j$ é apenas um identificador que informa de qual entrada $X_{j}, i \lambda_{l}^{j}$ é autovalor, note que pode ocorrer $d_{l}^{j} \neq d_{l}^{i}$ se $j \neq i$, e ainda $\sum_{l=1}^{r_{j}} d_{l}^{j}=n$ para todo $j$. Na notação de $\mathcal{O}_{X}$ contém um $\mathcal{D}_{X}$, isto é para enfatizar a ação diagonal de $S U(n)$ por $X$, 
ou seja, para todo $\xi \in \mathcal{O}_{X}$, existe $g \in S U(n)$ tal que

$$
\mathbf{A d}_{g}^{*} X=\left(\operatorname{Ad}_{g}^{*} X_{1}, \ldots, \operatorname{Ad}_{g}^{*} X_{m}\right)=\xi
$$

Agora considere a restrição $\left.\mathbf{A d}^{*}\right|_{S U(n) \times \mathcal{O}_{X}}$, pelo Teorema 2.23 temos que o mapa momento relacionado a esta ação é dado por

$$
\begin{aligned}
\mu: \mathcal{O}_{X} & \longrightarrow \mathfrak{s u}^{*}(n) \simeq \mathbb{R}^{n^{2}-1} \\
\xi & \longmapsto \mu(\xi)=\sum_{j=1}^{m} \xi_{j}
\end{aligned}
$$

onde $\xi=\left(\xi_{1}, \ldots, \xi_{m}\right)$. Note que tomando $0 \in \mathfrak{s u}^{*}(n)$, o conjunto de nível $\mu^{-1}(0) \subset \mathcal{O}_{X}$ é dado por

$$
\mu^{-1}(0)=\left\{\xi \in \mathcal{O}_{X} \mid \sum_{j=1}^{m} \xi_{j}=0\right\} .
$$

O produto interno em $\mathfrak{s u}(n)$ é dado por

$$
\langle X, Y\rangle=\frac{1}{2} \operatorname{tr}\left(X Y^{*}\right), X, Y \in \mathfrak{s u}(n)
$$

note que este produto é invariante pela ação adjunta, de fato

$$
\begin{aligned}
\left\langle\operatorname{Ad}_{g} X, \operatorname{Ad}_{g} Y\right\rangle & =\frac{1}{2} \operatorname{tr}\left(g X g^{*}\left(g Y g^{*}\right)^{*}\right) \\
& =\frac{1}{2} \operatorname{tr}\left(g X Y^{*} g^{*}\right) \\
& =\frac{1}{2} \operatorname{tr}\left(X Y^{*}\right) \\
& =\langle X, Y\rangle
\end{aligned}
$$

fizemos uso de $(A \cdot B)^{*}=B^{*} \cdot A^{*}$ e $g^{*} \cdot g=I$ pois $g \in S U(n)$. Sendo o produto interno em $\mathfrak{s u}(n)$ invariante pela ação adjunta e da equivalência das ações adjunta e coadjunta, veja Observação 8.19, temos que se $\xi \in \mathcal{O}_{X}$, então $\left\|\xi_{j}\right\|=\left\|X_{j}\right\|=r_{j} \in \mathbb{R}_{+}$para todo $j=1, \ldots, m$. Assim, $\mu^{-1}(0)$ descreve o espaço dos $m$-gons de lados $v_{j}$ de comprimentos fixados $r_{j}$, onde estamos fazendo a correspondência

$$
\xi_{j} \in \mathfrak{s u}(n) \longleftrightarrow v_{j} \in \mathbb{S}^{n^{2}-2} \subset \mathbb{R}^{n^{2}-1} .
$$

Olhando para apenas uma das entradas $\xi_{j}$ e a órbita de $S U(n)$ por $\xi_{j}$, a partir da correspondência (6.10) temos que para $n>2$, nem todo ponto de $\mathbb{S}^{n^{2}-2}$ pode ser alcançado por um vetor $\bar{v}_{j}$, onde $\bar{v}_{j}$ é o vetor que corresponde $\operatorname{Ad}_{g} \xi_{j}$, para $g \in S U(n)$, de fato, caso $n$ seja ímpar, teríamos uma esfera de dimensão ímpar 
e caso $n$ seja par, teríamos uma esfera de dimensão par maior que dois, logo em nenhum dos casos seria simplética, mas a órbita coadjunta é simplética, daí a órbita não ser toda a esfera. Uma outra forma de justificar que a órbita não é a esfera $\mathbb{S}_{r_{j}}^{n^{2}-2}$ é a seguinte, sabemos que para todo $\xi_{j} \in \mathcal{O}_{X_{j}},\left\|\xi_{j}\right\|=\left\|X_{j}\right\|$ e $\xi_{j}$ tem os mesmos autovalores de $X_{j}$, é fácil ver que sempre haverá $v_{h} \in \mathbb{S}_{r_{j}}^{n^{2}-2}$ associado a uma matriz $X_{h} \in \mathfrak{s u}(n)$, mas os autovalores de $X_{h}$ são distintos de $X_{j}$, ou seja, $X_{h} \notin \mathcal{O}_{X_{j}}$, portanto $\mathcal{O}_{X_{j}}$ não é a esfera $\mathbb{S}_{r_{j}}^{n^{2}-2}$, assim, $\mathcal{O}_{X_{j}} \subsetneq \mathbb{S}_{r_{j}}^{n^{2}-2}$.

Retornando a $\mu^{-1}(0)$, é provável que existam $\xi, \xi^{\prime} \in \mu^{-1}(0)$ de tal forma que $\xi^{\prime}=\operatorname{Ad}_{g} \xi$ para algum $g \in S U(n)$, afim de ter esses elementos identificados, em uma mesma classe, fazemos então o quociente

$$
M_{r}=\mu^{-1}(0) / S U(n), r=\left(r_{1}, \ldots, r_{m}\right) \in \mathbb{R}_{+}^{m}
$$

que é o espaço dos $m$-gons de lados $v_{j}$ de comprimentos fixados $r_{j}$ e é chamado espaço de polígonos. 


\section{Estratificação de Poisson}

Neste capítulo iremos estudar ações de grupos de Lie $G$ em uma variedade de Poisson $M$, e assumiremos que a ação é uma ação de Poisson própria. É bem conhecido que se a ação é própria e livre os espaços reduzidos $M / G$ e $M / / G$ são variedades, no entanto, se a ação não é livre esses espaços podem nem mesmo ser variedades, mas ainda são espaços de Hausdorff mesmo que $G$ e $M$ não sejam, e além disso, possuem uma estrutura de variedade estratificada, esta será a direção tomada neste trabalho sempre que a ação não for livre. Afim de entendermos o que ocorre com esses espaços enunciaremos alguns resultados e definições que se fazem necessários.

Definição 7.1 Dois subgrupos $H_{1}$ e $H_{2}$ de $G$ são ditos conjugados em $G$ se $H_{2}=g H_{1} g^{-1}$ para algum $g \in G$. Isto forma uma relação de equivalência no conjunto dos subgrupos de $G$. As classes de equivalência são chamadas de classes de conjugação. A classe de conjugação de um subgrupo $H$ em $G$ será denotada por $(H)$.

Definição 7.2 Considere a ação do grupo $G$ na variedade $M$. Dois pontos $x, y \in M$ são ditos do mesmo tipo se seus estabilizadores $G_{x}$ e $G_{y}$ são subgrupos conjugados em $G$. Isto define uma relação de equivalência em $M$, e as classes de equivalência $M_{(H)}$, onde $H$ é subgrupo de $G$, são chamadas subvariedades com tipo de órbita $(H)$.

A ação de um grupo $G$ em uma variedade $M$, naturalmente induz em $M$ e em $M / G$ uma partição por meio das chamadas subvariedades com tipo de órbita $(H)$.

O conceito de subvariedade com tipo de órbita $(H)$, onde $H$ é um subgrupo de $G$, foi mencionado acima, iremos agora formalizar esse conceito e introduzir outras definições importantes.

Definição 7.3 Seja $G$ um grupo de Lie agindo em uma variedade $M$. Considere $H$ um subgrupo fechado de G. Definimos as seguintes classes:

1. Classe de conjugação de $H$

$$
(H)=\left\{L \in G \mid L=g H g^{-1}, g \in G\right\}
$$


2. Subvariedade com tipo de órbita $(H)$

$$
M_{(H)}=\left\{m \in M \mid G_{m} \in(H)\right\}
$$

3. Subvariedade com tipo de isotropia $H$

$$
M_{H}=\left\{m \in M \mid G_{m}=H\right\}
$$

4. Subvariedade dos pontos fixados por $H$

$$
M^{H}=\left\{m \in M \mid H \subseteq G_{m}\right\}
$$

Observação 7.4 As componentes conexas das subvariedades $M_{H}, M^{H}$ e $M_{(H)}$ podem ser variedades de dimensões diferentes.

Claramente temos que $M_{H} \subset M_{(H)}$, uma vez que para todo $m \in M_{H}$ temos $G_{m}=H \in(H)$, além disto $G \cdot M_{H}=M_{(H)}$, dessa forma $M_{(H)}$ é o menor subconjunto $G$-invariante de $M$ que contém $M_{H}$.

Existe uma relação entre as subvariedades $M_{H}, M^{H}$ e $M_{(H)}$, a qual é descrita a seguir

Proposição 7.5 Considere que o grupo de Lie G age em $M$ propriamente. As subvariedades $M_{H}, M^{H}$ e $M_{(H)}$, como definidas acima, satisfazem a relação

$$
M_{H}=M_{(H)} \cap M^{H}
$$

Proof. Das definições de $M_{H}, M^{H}$ e $M_{(H)}$ percebemos claramente a inclusão $M_{H} \subset M_{(H)} \cap M^{H}$. Para a outra inclusão, seja $m \in M_{(H)} \cap M^{H}$, como $m \in M_{(H)}$, dessa forma $G_{m}$ é conjugado de $H$ em $G$, o fato de $m \in M^{H}$ nos diz que $H \subseteq G_{m}$. Como a ação de $G$ é própria segue que $G_{m}$ é compacto, resultado que será provado na proposição a seguir. Como $H$ é conjugado a $G_{m}$, temos que $H$ é também compacto. Consequentemente $H$ tem a mesma dimensão e o mesmo número de componentes conexas de $G_{m}$. Desde que $H \subseteq G_{m}$, obtemos que $H=G_{m}$, isto é, $m \in M_{H}$. Consequentemente, $M_{H}=M_{(H)} \cap M^{H}$.

A seguir provaremos que pontos em uma mesma órbita da ação de $G$ em $M$ possuem subgrupos de isotropia conjugados, isto significa que esses pontos estão em uma mesma subvariedade com tipo de órbita $(H)$.

Proposição 7.6 O grupo de isotropia $G_{g \cdot m}, m \in M$, é conjugado ao grupo de isotropia $G_{m}$ pelo elemento $g \in G$. 
Proof. Temos que

$$
h \in G_{g \cdot m} \Leftrightarrow h \cdot(g \cdot m)=g \cdot m \Leftrightarrow\left(g^{-1} h g\right) \cdot m=m \Leftrightarrow g^{-1} h g \in G_{m}
$$

portanto

$$
g^{-1} G_{g \cdot m} g=G_{m} \text { equivalentemente } G_{g \cdot m}=g G_{m} g^{-1}
$$

Uma reformulação dessa proposição é o seguinte corolário.

Corolário 7.7 Considere a ação de $G$ na variedade $M$, se $x, y \in M$ estão em uma mesma órbita, então $x, y \in M_{(H)}$, onde $H$ é subgrupo de isotropia de $x$ ou de $y$.

O resultado a seguir mostra que subgrupos de isotropia de ações próprias são sempre compactos. Lembrando que uma aplicação $f: M \longrightarrow N$ entre dois espaços topológicos é própria se a pré imagem de todo conjunto compacto em $N$ é compacto em $M$.

Proposição 7.8 Seja $\psi: G \times M \rightarrow M$ uma ação própria do grupo de Lie $G$ na variedade $M$, então para qualquer $m \in M$, o subgrupo de isotropia $G_{m}$ é compacto.

Proof. Considere a seguinte aplicação própria

$$
\begin{aligned}
\varphi: G \times M & \longrightarrow M \times M \\
(g, m) & \longmapsto \varphi(g, m)=(m, \psi(g, m))
\end{aligned}
$$

note que

$$
\varphi^{-1}(\{m\} \times M)=G \times\{m\}
$$

assim a aplicação

$$
\begin{aligned}
G \times\{m\} & \longrightarrow\{m\} \times M \\
(g, m) & \longmapsto(m, g \cdot m)
\end{aligned}
$$

é própria, consequentemente, também é própria a aplicação

$$
\begin{aligned}
\varphi_{m}: G & \longrightarrow M \\
g & \longmapsto \varphi_{m}(g):=g \cdot m
\end{aligned}
$$

portanto $\varphi_{m}^{-1}(m)=G_{m}$ é compacto.

Observação 7.9 Poderia ter sido utilizado a Proposição 21.5 de caracterização de ações próprias de [14], item (c), bastando tomar para o caso $K=\{m\} \subseteq M$. 
Nesta tese utilizaremos essencialmente as ações dos grupos de Lie $S U(2)$ e $S U(n)$, os quais são grupos compactos, dessa forma as ações nas variedades são todas próprias e além disso, a Proposição 7.8 garante que os subgrupos de isotropia dessas ações são compactos, poderíamos justificar isto apenas usando o fato de serem subgrupos fechados.

A partir de agora iremos tratar a respeito da estratificação de um espaço topológico para depois definir as estratificações simplética e de Poisson, as quais serão úteis para o entendimento dos espaços reduzidos $M / G$ e $M / / G$.

Definição 7.10 Sejam $X$ um espaço topológico e $I$ um conjunto de índices. Uma coleção $\left\{X_{i}\right\}_{i \in I}$ de subconjuntos de $X$ é chamada localmente finita se, $e$ somente se, cada $x \in X$ tiver uma vizinhança $U$ tal que $U \cap X_{i} \neq \emptyset$ para no máximo uma quantidade finita $i \in I$. E ainda, $X_{i}$ é um subconjunto localmente fechado de $X$ se para todo $x \in X_{i}$ existe uma vizinhança aberta $V$ de $x$ em $X$ tal que $V \cap X_{i}$ é um subconjunto fechado de $V$.

Definição 7.11 Uma estratificação de um espaço topológico $X$ é uma partição localmente finita de $X$ em variedades conexas localmente fechadas $M_{i}(i \in I)$, as quais são chamadas de estrata, tal que para cada $i \in I$ o fecho de $M_{i}$ é igual a $M_{i} \cup \bigcup_{j \in I_{i}} M_{j}$, onde $I_{i} \subset I-\{i\}$, e $\operatorname{dim} M_{j}<\operatorname{dim} M_{i}$ para cada $j \in I_{i}$.

Em [1], consta na Definição 2.7.3 a definição de estratificação de Whitney.

Teorema 7.12 Se $G$ age propriamente em $M$, as componentes conexas das subvariedades com tipo de órbita $(H), c\left(M_{(H)}\right)$, formam uma estratificação de $M$. Além disso, cada quociente $M_{(H)} / G$ tem uma estrutura suave única fazendo $M_{(H)} \rightarrow M_{(H)} / G$ uma submersão. As componentes conexas do quociente $M_{(H)} / G$ formam uma estratificação do espaço quociente $M / G$.

A demonstração da primeira parte deste resultado pode ser encontrada em [1], Teorema 2.7.4.

Em notação o Teorema 7.12 nos diz que as partições $\left\{c_{i}\left(M_{\left(H_{j}\right)}\right)\right\}_{i \in I}, j \in$ $\mathbb{N}$ e $\left\{c_{i}\left(M_{\left(H_{k}\right)} / G\right)\right\}_{i \in I^{\prime}}, k \in \mathbb{N}$ formam uma estratificação de $M$ e do espaço quociente $M / G$, respectivamente, onde $H_{j}$ são subgrupos de $G$.

Afim de compreendermos alguns resultados a seguir, precisaremos de algumas ferramentas básicas de álgebra, dentre elas o normalizador de um subgrupo e o subgrupo normal de um grupo.

Para qualquer subgrupo $H$ de $G$, o normalizador de $H$ em $G$ é definido como

$$
N(H):=\left\{g \in G \mid g H g^{-1}=H\right\}
$$

Proposição 7.13 O normalizador $N(H)$ definido acima satisfaz os seguintes: 
1. $N(H)$ é um subgrupo de $G$.

2. $H$ é subgrupo de $N(H)$.

3. $N(H)$ é o subgrupo maximal de $G$ que contém $H$ como subgrupo normal.

Proof. Claramente $N(H)$ é não vazio pois $e \in N(H)$. Agora sejam $g, h \in$ $N(H)$

$$
\begin{aligned}
(g h) H(g h)^{-1} & =g\left(h H h^{-1}\right) g^{-1} & & \\
& =g H g^{-1} & & \text { pois } h \in N(H) \\
& =H & & \text { pois } g \in N(H)
\end{aligned}
$$

dessa forma $g h \in N(H)$, assim $N(H)$ é fechado para a operação do grupo. Desde que $g \in N(H)$, temos que $g H g^{-1}=H$, dessa forma, fazendo a conjugação por $g^{-1}$ em ambos os lados, obtemos

$$
H=g^{-1} H g \Rightarrow g^{-1} \in N(H)
$$

logo $N(H)$ é fechado para a inversão, portanto $N(H)$ é subgrupo de $G$ e o item 1 está assim demonstrado.

Como $H$ é subgrupo de $G$, logo tem estrutura de grupo, falta apenas mostrar que $H \subseteq N(H)$. Como $H$ é grupo, dados $a, b \in H, a b \in H$ e $a^{-1} \in H$. Dado $a \in H$, precisamos provar que $a H a^{-1}=H$ para todo $a \in H$. Para a inclusão $a H a^{-1} \subseteq H$, seja $a h a^{-1} \in a H a^{-1}$, desde que $H$ é fechado para a operação do grupo, segue que $a h a^{-1} \in H$. Para a outra inclusão, $H \subseteq a H a^{-1}$, seja $h \in H$, desde que $H$ é fechado para a operação do grupo, $a^{-1} h a \in H$, dessa forma

$$
h=a\left(a^{-1} h a\right) a^{-1} \in a H a^{-1}
$$

provando assim a segunda inclusão, logo $a H a^{-1}=H$. Portanto se $a \in H \Rightarrow a \in$ $N(H)$ e portanto $H \subseteq N(H)$, dessa forma $H$ é subgrupo de $N(H)$, provando assim o item 2.

Seja $K \subset G$ um subgrupo tal que $H$ é subgrupo normal de $K$, então para todo $x \in K$ temos que

$$
x H x^{-1}=H
$$

da definição de $N(H)$ segue que $x \in N(H)$, e portanto $K \subset N(H)$, demonstrando assim o item 3.

O próximo resultado nos fornece uma relação importante entre o normalizador $N(H)$ e a subvariedade com tipo de isotropia $H$. 
Proposição 7.14 Seja $H$ um subgrupo fechado de $G$ tal que $M_{H}$ é não vazio. Para cada $g \in G$ temos

$$
g \in N(H) \Leftrightarrow g \cdot M_{H}=M_{H} \Leftrightarrow\left(g \cdot M_{H}\right) \cap M_{H} \neq \emptyset
$$

onde estamos considerando que $G$ age na variedade $M$.

Proof. Se $m \in M_{H}$, então $G_{m}=H$. Pela Proposição 7.6 temos que $g \cdot m \in M_{H}$, isto é, $G_{g \cdot m}=H=G_{m}$ se, e somente se, $g \in N(H)$, o que demonstra o resultado.

Pelo mostrado na Proposição 7.13, $N(H)$ é subgrupo fechado de $G$ e consequentemente é um grupo de Lie, e uma vez que $H$ é um subgrupo normal de $N(H)$ segue da Proposição 8.2 que $N(H) / H$ é também um grupo de Lie. A Proposição 7.14 nos diz que o maior subconjunto de $G$ que deixa $M_{H}$ invariante coincide com o subgrupo normalizador $N(H)$ de $H$ em $G$, isto é, $N(H)$ age em $M_{H}$, o que é justificado por $g \cdot M_{H}=M_{H} \Leftrightarrow g \in N(H)$, além disso, esta ação induz uma ação livre do grupo quociente $N(H) / H$ em $M_{H}$.

Nos dois resultados a seguir constam subvariedades Poisson-Dirac e Lie-Dirac, conceitos que não serão abordados neste trabalho. Em [28], Fernandes prova o seguinte

Teorema 7.15 Seja $G \times M \longrightarrow M$ uma ação de Poisson própria. Então o conjunto dos pontos fixados $M^{G}$ é uma subvariedade Poisson-Dirac, onde $M^{G}=\{p \in M \mid g \cdot p=p, \forall g \in G\}$

Em [10], Fernandes, Ortega e Ratiu mostraram que para um grupo de Lie compacto tem-se

Teorema 7.16 Seja G um grupo de Lie compacto e $M$ um G-espaço de Poisson $^{1}$. Então $M^{G}$ é uma subvariedade Lie-Dirac de $M$ com colchete de Poisson $\{\cdot, \cdot\}_{M^{G}}$ dado por

$$
\{f, h\}_{M^{G}}:=\left.\{\tilde{f}, \tilde{h}\}\right|_{M^{G}}, f, h \in \mathcal{C}^{\infty}\left(M^{H}\right)
$$

onde $\tilde{f}, \tilde{h} \in \mathcal{C}^{\infty}(M)^{G}$ denota extensões $G$-invariantes arbitrárias de $f, h \in$ $\mathcal{C}^{\infty}\left(M^{G}\right)$.

Nesse mesmo artigo, Fernandes, Ortega e Ratiu mostram que a subvariedade com tipo de isotropia $M_{H}$ é uma subvariedade Lie-Dirac de $M$, como não é o intuito deste trabalho apresentar um aprofundamento nos conceitos de

${ }^{1}$ Veja a Definição 4.27 
variedades Poisson-Dirac e Lie-Dirac, no resultado a seguir, o qual foi extraído de [10], nos limitamos a dizer que a subvariedade $M_{H}$ é uma subvariedade de Poisson, os resultados acima foram enunciados em sua forma original em respeito aos pesquisadores mencionados e ao caro leitor que possa ter interesse em ir mais a fundo nesse conhecimento.

Proposição 7.17 Sejam $\psi: G \times M \rightarrow M$ uma ação de Poisson própria, $H \subset G$ um grupo de isotropia e $N(H)$ o normalizador de $H$ em $G$. Então:

1. $M_{H}$ é uma subvariedade de Poisson de $M$ com colchete de Poisson dado por

$$
\{f, h\}_{M_{H}}=\left.\{\tilde{f}, \tilde{h}\}\right|_{M_{H}}, f, h \in \mathcal{C}^{\infty}\left(M_{H}\right)
$$

onde $\tilde{f}, \tilde{h} \in \mathcal{C}^{\infty}(M)^{H}$ denota quaisquer extensões $H$-invariantes das funções $f, h \in \mathcal{C}^{\infty}\left(M_{H}\right)$.

2. A ação natural de $L(H):=N(H) / H$ em $M_{H}$ é uma ação de Poisson própria e livre.

A demonstração deste resultado pode ser encontrada em [10].

Como mencionado em [10], o resultado acima é o análogo em geometria de Poisson de um resultado bem conhecido em simplética, sendo este devido a Guillemin e Sternberg ([11], Teorema 3.5) o qual mostra que as componentes conexas das variedades com tipo de isotropia $M_{H}$ são subvariedades simpléticas de $M$.

Como a ação de $L(H)$ em $M_{H}$ é uma ação de Poisson livre e própria, o Teorema 4.28 garante que o colchete de Poisson $\{\cdot, \cdot\}_{M_{H}}$ induz uma única estrutura de Poisson $\{\cdot, \cdot\}_{M_{H} / L(H)}$ no espaço de órbitas $M_{H} / L(H)$ que faz a projeção $\pi_{L}: M_{H} \rightarrow M_{H} / L(H)$ uma aplicação de Poisson.

Corolário 7.18 Considere a ação de Poisson livre e própria de $L(H)$ em $M_{H}$, temos assim $\left(M_{H}, \pi_{M_{H}}, L(H)\right)$ um $L(H)$-espaço de Poisson livre e próprio. Então existe uma única estrutura de Poisson quociente $\pi_{M_{H} / L(H)}$ em $M_{H} / L(H)$ para a qual a aplicação $q: M_{H} \rightarrow M_{H} / L(H)$ é Poisson.

Este resultado será utilizado na demonstração do Teorema 7.22 de estratificação de Poisson.

Do Teorema 7.12, temos que a decomposição $M=\bigcup_{(H)} M_{(H)}$ em subvariedades com tipo de órbita $(H)$ induz a decomposição

$$
X=\bigcup_{(H)} M_{(H)} / G
$$


do espaço de órbitas $X=M / G$. A estratificação suave de $X$ é então

$$
X=\bigcup_{i \in I} X_{i}
$$

onde cada $X_{i}$ é uma componente conexa de algum $M_{(H)} / G$. A álgebra de funções suaves no espaço de órbitas $X$ é

$$
\mathcal{C}^{\infty}(X)=\left\{f \in \mathcal{C}^{0}(M / G) \mid f \circ \pi_{G} \in \mathcal{C}^{\infty}(M)^{G}\right\}
$$

onde $\pi_{G}$ é dado pela composição $\pi_{G}=i \circ F_{H} \circ \pi_{L}$, com $\pi_{L}$ a projeção natural $M_{H} \rightarrow M_{H} / L(H), F_{H}: M_{H} / L(H) \longrightarrow M_{(H)} / G$ sendo o difeomorfismo fornecido na Proposição 7.21 e $i$ a inclusão $M_{(H)} / G \hookrightarrow M / G$. O diagrama a seguir mostra estas aplicações

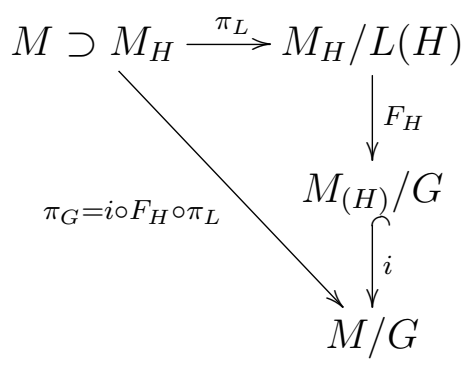

Na Definição 7.11 foi dada a estratificação de um espaço topológico, agora serão dadas as definições das estratificações de Poisson e Simplética, respectivamente.

Definição 7.19 Seja X um espaço topológico. Uma estratificação de Poisson de $X$ é uma estratificação suave $\mathcal{S}=\left\{S_{i}\right\}_{i \in I}$ de $X$ junto com uma álgebra de Poisson $\left(\mathcal{C}^{\infty}(X),\{\cdot, \cdot\}_{X}\right)$, onde $\mathcal{C}^{\infty}(X) \subset \mathcal{C}^{0}(X)$ é o espaço das funções suaves, tal que:

1. Cada estrata $S_{i}, i \in I$, é uma variedade de Poisson.

2. As inclusões $i: S_{i} \hookrightarrow X$ são aplicações de Poisson, isto é,

$$
\{f, h\}_{X} \circ i=\{f \circ i, h \circ i\}_{S_{i}}, f, h \in \mathcal{C}^{\infty}(X) .
$$

As estruturas de Poisson nas estratas $S_{i}$ são unicamente determinadas pela álgebra de Poisson $\left(\mathcal{C}^{\infty}(X),\{\cdot, \cdot\}_{X}\right)$.

Definição 7.20 Um espaço simplético estratificado $X$ é um espaço estratificado com uma estrutura suave $\mathcal{C}^{\infty}(X)$ tal que:

1. Cada estrata $S_{i}$ é uma variedade simplética. 
2. $\mathcal{C}^{\infty}(X)$ é uma álgebra de Poisson.

3. Os mergulhos $S_{i} \hookrightarrow X$ são Poisson.

Temos que $M_{H} / L(H)$ é uma variedade de Poisson, o resultado a seguir, o qual encontra-se em [10], garante que $M_{(H)} / G$ herda uma estrutura de Poisson de $M_{H} / L(H)$ e essa estrutura independe do subgrupo de isotropia tomado na classe de conjugação de $H$.

Proposição 7.21 Sejam $\psi: G \times M \rightarrow M$ uma ação de Poisson própria e $H \subset G$ um grupo de isotropia.

(i) A aplicação natural $F_{H}: M_{H} / L(H) \rightarrow M_{(H)} / G$ é um difeomorfismo, assim $M_{(H)} / G$ herda uma estrutura de Poisson de $M_{H} / L(H)$.

(ii) Se $H_{1}, H_{2} \in(H)$ são grupos de isotropia conjugados, as estruturas de Poisson em $M_{(H)} / G$ induzidas por $M_{H_{1}} / L\left(H_{1}\right)$ e $M_{H_{2}} / L\left(H_{2}\right)$ coincidem.

O Teorema 7.12 garante que as componentes conexas de $M_{(H)} / G$ formam uma estratificação do espaço quociente $M / G$, enquanto que a proposição anterior garante que $M_{(H)} / G$ herda uma estrutura de Poisson, o resultado a seguir nos fornece a estratificação de Poisson do espaço de órbitas $M / G$, este resultado foi extraído de [10] e é devido a Fernandes, Ortega e Ratiu e é conhecido como o Teorema de estratificação de Poisson.

Teorema 7.22 Seja $\psi: G \times M \rightarrow M$ uma ação de Poisson própria. As componentes conexas do espaço reduzido $M_{(H)} / G$ formam uma estratificação de Poisson de $\left(M / G,\{\cdot, \cdot\}_{M / G}\right)$.

Proof. A estratificação é garantida pelo Teorema 7.12 o qual mostra que as componentes conexas de $M_{(H)} / G$ formam uma estratificação de $M / G$, falta provar que a aplicação inclusão

$$
i: M_{(H)} / G \hookrightarrow M / G
$$

é Poisson, ou seja, satisfaz

$$
\{f, h\}_{M / G} \circ i=\{f \circ i, h \circ i\}_{M_{(H)} / G}, f, h \in \mathcal{C}^{\infty}(M / G) .
$$


Consideramos o isomorfismo $F_{H}: M_{H} / L(H) \longrightarrow M_{(H)} / G$ e a projeção $\pi_{L}: M_{H} \longrightarrow M_{H} / L(H)$, então para qualquer $m \in M_{H}$, temos:

$$
\begin{aligned}
\{f \circ i, h \circ i\}_{M_{(H)} / G}\left(F_{H}([m])\right) & =\left\{f \circ i \circ F_{H}, h \circ i \circ F_{H}\right\}_{M / L(H)}\left(\pi_{L}(m)\right) \\
& =\left\{f \circ i \circ F_{H} \circ \pi_{L}, h \circ i \circ F_{H} \circ \pi_{L}\right\}_{M_{H}}(m) \\
& =\left\{f \circ \pi_{G}, h \circ \pi_{G}\right\}_{M}(m) \\
& =\{f, h\}_{M / G} \circ \pi_{G}(m) \\
& =\{f, h\}_{M / G} \circ i\left(F_{H}\left(\pi_{L}(m)\right)\right) \\
& =\{f, h\}_{M / G} \circ i\left(F_{H}([m])\right)
\end{aligned}
$$

$f \circ \pi_{G}, h \circ \pi_{G} \in \mathcal{C}^{\infty}(M)$ são $G$-invariantes, e portanto $H$-invariantes, extensões de $f \circ i \circ F_{H} \circ \pi_{L}, h \circ i \circ F_{H} \circ \pi_{L} \in \mathcal{C}^{\infty}\left(M_{H}\right)$.

A proposição a seguir nos mostra uma relação interessante a respeito das órbitas coadjuntas de um grupo de Lie compacto e conexo e as estratas do espaço de órbitas.

Proposição 7.23 Seja G um grupo de Lie compacto e conexo agindo no dual de sua álgebra de Lie $\mathfrak{g}^{*}$ pela ação coadjunta. O espaço $\mathfrak{g}^{*} / G$ é um espaço estratificado de Poisson onde as estratas são dadas pelas componentes conexas de $\mathfrak{g}_{(H)}^{*} / G$ que são órbitas coadjuntas, onde $H$ é subgrupo de isotropia da ação.

Proof. Desde que $G$ é um grupo de Lie compacto, segue do Corolário 6.10 que a ação é uma ação própria, a ação é a coadjunta e foi provado na Proposição 4.15 que esta é uma ação de Poisson, portanto, o Teorema 7.12 garante que $\mathfrak{g}^{*} / G$ é um espaço estratificado cujas estratas são dadas pelas componentes conexas de $\mathfrak{g}_{(H)}^{*} / G$ onde $H$ é subgrupo de isotropia da ação coadjunta, o Teorema 7.22 garante que essa estratificação é uma estratificação de Poisson.

Resta provar que $c\left(\mathfrak{g}_{(H)}^{*} / G\right)=\mathcal{O}_{\xi}$, onde $\xi \in \mathfrak{g}^{*}$ e $\mathcal{O}_{\xi}$ denota a órbita coadjunta de $G$ por $\xi$. Para esta última parte, seja $H$ um subgrupo de isotropia para a ação coadjunta de $G$, temos que

$$
\mathfrak{g}_{H}^{*}=\left\{\xi \in \mathfrak{g}^{*} \mid G_{\xi}=H\right\} \text { e } \mathfrak{g}_{(H)}^{*}=\left\{x \in \mathfrak{g}^{*} \mid G_{x} \in(H)\right\}
$$

assim, se $x \in \mathfrak{g}_{(H)}^{*}$ então existe $g \in G$ tal que $G_{x}=g H g^{-1}$, sendo $G$ um grupo, existe em $G$ o inverso de $g$, que denotamos por $g^{-1}$, daí $y=\operatorname{Ad}_{g^{-1}}^{*} x \in \mathfrak{g}_{H}^{*}$, dessa forma $y$ e $x$ estão em uma mesma órbita do grupo $G$, seja $\mathcal{O}_{x}$ essa órbita, desde que $G$ é conexo, $\mathcal{O}_{x}$ também o é, além disso, sabemos que $\mathfrak{g}_{H}^{*} \subset \mathfrak{g}_{(H)}^{*}$. Portanto, para todo $x \in \mathfrak{g}_{(H)}^{*}, \mathcal{O}_{x} \in \mathfrak{g}_{(H)}^{*} / G$, mais precisamente $\mathcal{O}_{x}$ é uma componente conexa de $\mathfrak{g}_{(H)}^{*} / G$. Por outro lado, dado qualquer componente conexa de $\mathfrak{g}_{(H)}^{*} / G, c\left(\mathfrak{g}_{(H)}^{*} / G\right)$, naturalmente existe $x \in \mathfrak{g}_{(H)}^{*}$ que está nessa 
componente conexa, pelo argumento dado anteriormente, concluímos que $x$ está em uma órbita a qual é conexa, isto conclui a demonstração

Dado um grupo de Lie compacto e conexo $G$, considere a variedade $M=\prod_{j=1}^{m} \mathfrak{g}^{*}$ a qual é dotada com a estrutura de Poisson produto. Considere ainda a ação coadjunta diagonal de $G$ em $M$, esta é uma ação própria pois $G$ é compacto, mas nada nos garante que a ação seja livre, dessa forma, seja $H$ um subgrupo de isotropia dessa ação, podemos determinar em $M$ uma subvariedade de Poisson, a qual é a chamada subvariedade com tipo de isotropia $H$, dada por

$$
M_{H}=\left\{X \in M \mid G_{X}=H\right\}
$$

ou seja, sendo $X=\left(X_{1}, \ldots, X_{m}\right)$ temos que se $X_{j} \neq 0$ então $H \subset G_{X_{j}}$, note que $X$ pode ter até $(n-1)$ entradas nulas, caso seja esse o caso, teremos que o subgrupo de isotropia da entrada não nula é exatamente o subgrupo $H$. Temos ainda que em $M$ existe a subvariedade com tipo de órbita $(H)$, a qual é dada por

$$
M_{(H)}=\left\{X \in M \mid G_{X} \in(H)\right\} .
$$

Em $G$ existe um subgrupo maximal que possui $H$ como subgrupo normal, esse subgrupo é chamado de normalizador de $H$ em $G$, podemos ainda obter um outro grupo através do quociente $L(H)=N(H) / H$, este é um subgrupo que age própria e livremente em $M_{H}$, o que nos garante assim que $M_{H} / L(H)$ admite uma estrutura de Poisson, uma vez que existe o difeomorfismo

$$
F_{H}: M_{H} / L(H) \longrightarrow M_{(H)} / G
$$

segue que $M_{(H)} / G$ herda uma estrutura de Poisson.

É possível que existam outros subgrupos de isotropia para essa ação que não pertençam a classe de conjugação de $H$, dessa forma, sejam $\left\{H_{j}\right\}_{j \in I}$ esses subgrupos, o que nos possibilita obter os quocientes $M_{\left(H_{j}\right)} / G$ os quais possuem uma estrutura de Poisson, e portanto, de acordo com o Teorema 7.22, a estratificação de Poisson do espaço de órbitas $M / G$ é realizada pelas componentes conexas dos quocientes $M_{\left(H_{k}\right)} / G$, onde $H_{k}$ é subgrupo de isotropia da ação.

Dado qualquer $X \in M$ temos que a órbita coadjunta diagonal de $G$ por $X$ é dada por

$$
\mathcal{O}_{X}^{\mathcal{D}}=\mathcal{D}_{X}\left(\prod_{j=1}^{m} \mathcal{O}_{X_{j}}\right)
$$


isto significa que para todo $Y \in \mathcal{O}_{X}^{\mathcal{D}}$ existe $g \in G$ tal que

$$
\operatorname{Ad}_{g}^{*} X=\left(\operatorname{Ad}_{g}^{*} X_{1}, \ldots, \operatorname{Ad}_{g}^{*} X_{m}\right)=Y
$$

$\mathcal{O}_{X_{j}}$ denota a órbita coadjunta de $G$ por $X_{j} \in \mathfrak{g}^{*}$ para todo $j$. Vale ressaltar que se $G$ é conexo então $c\left(M_{\left(H_{k}\right)} / G\right)=\mathcal{O}_{X}^{\mathcal{D}}$ para algum $X \in M$.

Façamos agora um resultado semelhante ao da proposição anterior, desta vez para a ação coadjunta diagonal de um grupo de Lie em um produto de duais de álgebras de Lie.

Proposição 7.24 Seja G um grupo de Lie compacto e conexo agindo na variedade de Poisson $M=\prod_{j=1}^{m} \mathfrak{g}^{*}$ pela ação coadjunta diagonal. O espaço $M / G$ é um espaço estratificado de Poisson onde as estratas são dadas pelas componentes conexas de $M_{(H)} / G$ as quais são realizadas pelas órbitas coadjuntas diagonais $\mathcal{O}_{x}^{\mathcal{D}}$, onde $H$ é subgrupo de isotropia da ação.

Proof. A demonstração é semelhante a proposição anterior com pequenas alterações. Desde que $G$ é um grupo de Lie compacto, segue do Corolário 6.10 que a ação é uma ação própria, a ação é a coadjunta diagonal, a qual foi provada na Proposição 4.19 que é uma ação de Poisson, portanto, o Teorema 7.12 garante que $M / G$ é um espaço estratificado cujas estratas são dadas pelas componentes conexas de $M_{(H)} / G$ onde $H$ é subgrupo de isotropia da ação coadjunta diagonal, o Teorema 7.22 garante que essa estratificação é uma estratificação de Poisson.

Seja $H$ um subgrupo de isotropia, temos que a subvariedade com tipo de isotropia $H$ é dada por

$$
M_{H}=\left\{\xi \in M \mid G_{\xi}=H\right\}
$$

sendo $\xi=\left(\xi_{1}, \ldots, \xi_{m}\right), H$ estabiliza todas as entradas de $\xi$, logo $H \subset G_{\xi_{j}}$ para todo $j$. A subvariedade com tipo de órbita $(H)$ é dada por

$$
M_{(H)}=\left\{x \in M \mid G_{x} \in(H)\right\}
$$

isto significa que se $x \in M_{(H)}$ então existe $g \in G$ tal que $G_{x}=g H g^{-1}$, logo existe $\xi \in M_{H}$ tal que $\operatorname{Ad}_{g^{-1}}^{*} \xi=\left(\operatorname{Ad}_{g^{-1}}^{*} \xi_{1}, \ldots, \operatorname{Ad}_{g^{-1}}^{*} \xi_{m}\right)=x$, dessa forma $\mathcal{O}_{x}^{\mathcal{D}} \subset M_{(H)}$ onde $\mathcal{O}_{x}^{\mathcal{D}}$ denota a órbita coadjunta diagonal de $G$ por $x$, portanto se $A \in M_{(H)} / G$ então $A=\mathcal{O}_{x}^{\mathcal{D}}$ para $x \in M_{(H)}$, poderíamos ainda mencionar para $\xi \in M_{H}$, o qual sempre existe em $\mathcal{O}_{x}^{\mathcal{D}}$, uma vez que $G$ é conexo, temos que $\mathcal{O}_{x}^{\mathcal{D}}$ é conexa, portanto $c\left(M_{(H)} / G\right)=\mathcal{O}_{x}^{\mathcal{D}}$ para algum $x$. Portanto $\mathcal{O}_{x}^{\mathcal{D}}$ é uma estrata da estratificação de $M / G$. 
Considere uma ação de um grupo de Lie $G$ em uma variedade $M$. Uma consequência da Proposição 7.6 é a seguinte: Dado um subgrupo de isotropia $H \subset G$, temos que dado qualquer $X \in M_{H}$, a órbita de $G$ passando por $X$, $\mathcal{O}_{X}$, é um subconjunto da subvariedade com tipo de órbita $(H), \mathcal{O}_{X} \subset M_{(H)}$, vale ressaltar que temos uma inclusão e não necessariamente uma igualdade, isto é devido a possibilidade de pontos distintos de $M_{H}$ estarem em órbitas distintas. No caso do grupo $S U(n)$ agindo pela ação coadjunta em $\mathfrak{s u}(n)$, se considerarmos como subgrupo de isotropia o toro $\mathbb{T} \subset S U(n)$, na subvariedade com tipo de isotropia $\mathbb{T}, \mathfrak{s u}^{*}(n)_{\mathbb{T}}$, certamente existem pontos $X, Y \in \mathfrak{s u}(n)_{\mathbb{T}}$ onde os autovalores de $X$ são diferentes dos autovalores de $Y, \log O X$ e $Y$ estão em órbitas distintas, mas devido as multiplicidades serem todas iguais a 1 segue que

$$
\mathcal{O}_{X} \simeq \mathbb{F}_{\mathbb{C}}(1, \ldots, 1) \text { e } \mathcal{O}_{Y} \simeq \mathbb{F}_{\mathbb{C}}(1, \ldots, 1)
$$

estas órbitas são difeomórficas e disjuntas. O mesmo ocorre se o subgrupo de isotropia não fosse o toro e sim o subgrupo $G_{d}$, poderíamos encontrar $X, Y \in$ $\mathfrak{s u}^{*}(n)_{G_{d}}$ tal que $X=i \operatorname{diag}\left(\lambda_{1} I_{d_{1}}, \ldots, \lambda_{r} I_{d_{r}}\right)$ e $Y=i \operatorname{diag}\left(\lambda_{1}^{\prime} I_{d_{1}}, \ldots, \lambda_{r}^{\prime} I_{d_{r}}\right)$ e ocorrer que para algum $j, \lambda_{j} \neq \lambda_{j}^{\prime}$, ou seja, teríamos

$$
\mathcal{O}_{X} \simeq \mathbb{F}_{\mathbb{C}}\left(d_{1}, \ldots, d_{r}\right) \text { e } \mathcal{O}_{Y} \simeq \mathbb{F}_{\mathbb{C}}\left(d_{1}, \ldots, d_{r}\right)
$$

com estas órbitas sendo difeomórficas no entanto disjuntas. Vale ressaltar que a ação de $S U(n)$ preserva a norma, dessa forma se $X, Y \in M_{H}$ de tal forma que $\|X\| \neq\|Y\|$ então $\mathcal{O}_{X} \cap \mathcal{O}_{Y}$ é vazia, e isto ocorre mesmo que esses pontos tenham a mesma sequência que descreve as multiplicidades de seus autovalores, ou seja, pode ocorrer (7.2) mas ainda serem disjuntas. Pode ainda ocorrer $\|X\|=\|Y\| \operatorname{mas} \mathcal{O}_{X} \cap \mathcal{O}_{Y}$ ser vazia, como é o caso mencionado acima.

Considere a ação $\psi: G \times M \longrightarrow M$, seja $H$ um subgrupo de isotropia dessa ação. Se $X \in M_{(H)}$, então $G_{X}=g H g^{-1}$, isso significa que $X \in \mathcal{O}_{\xi}$ para algum $\xi \in M_{H}$, portanto existe $g \in G$ tal que $\psi_{g}(\xi)=X$ ou equivalentemente $\psi_{g^{-1}}(X)=\xi$. Para o caso do grupo $S U(n)$, onde todo elemento de $\mathfrak{s u}^{*}(n)$ pode ser diagonalizado, pensar nas subvariedades com tipo de órbita $(H)$ é suficiente pensar nos elementos diagonais, pois em toda órbita tem um elemento dessa forma, logo estes elementos caracterizam as subvariedades com tipo de órbita $(H)$ nesse sentido.

Em alguns exemplos a seguir nos depararemos com o grupo de Weyl, o qual será o grupo $L(H)$ da Proposição 7.17 quando tomarmos $H$ como sendo um determinado grupo, mais especificamente, um toro maximal $\mathbb{T}$ em um grupo de Lie compacto e conexo $G$. O normalizador de $\mathbb{T}$ que é o subgrupo dado por $N(\mathbb{T})=\left\{g \in G \mid g \mathbb{T} g^{-1}=\mathbb{T}\right\}$, sabemos que $\mathbb{T}$ é subgrupo normal de $N(\mathbb{T})$ enquanto que o grupo quociente $W(G)=N(\mathbb{T}) / \mathbb{T}$ é chamado o grupo 
de Weyl de $G$.

\section{1}

\section{Exemplos}

Nos exemplos a seguir evidenciaremos os conceitos até aqui apresentados, com o intuito de entendermos a estratificação em cada caso e posteriormente sabermos como o espaço de polígonos pode ser realizado. Na primeira parte faremos o estudo usando o grupo de Lie $S U(2)$ e na segunda o $S U(n)$.

\subsection{1}

Estratificação das variedades $\mathfrak{s u}^{*}(2), \prod_{j=1}^{m} \mathfrak{s u}^{*}(2) \mathbf{e} \prod_{j=1}^{m} \mathbb{S}_{\left|\lambda_{j}\right|}^{2}$

Exemplo 7.25 Considere a ação coadjunta de $S U(2)$ em $\mathfrak{s u}^{*}(2)$. Já sabemos que as ações adjunta e coadjunta para o grupo $S U(2)$ são equivalentes, logo não faremos distinção entre elas, dessa forma, dado $X \in \mathfrak{s u}(2)$ existe $g \in S U(2)$ tal que

$$
H=\operatorname{Ad}_{g} X=i \operatorname{diag}(\lambda,-\lambda),
$$

sabemos que o estabilizador de $H$ é o subgrupo

$$
S U(2)_{H}=\mathbb{T}=\{g \in S U(2) \mid g=\operatorname{diag}(\alpha, \bar{\alpha}), \alpha \in U(1)\} .
$$

A órbita de $S U(2)$ pela ação coadjunta por $H$, como mostrado em (5.2), é dada por

$$
S U(2) \cdot H=g H g^{-1}=i \lambda\left(\begin{array}{cc}
|\alpha|^{2}-|\beta|^{2} & 2 \alpha \bar{\beta} \\
2 \bar{\alpha} \beta & |\beta|^{2}-|\alpha|^{2}
\end{array}\right)
$$

fixando $g \in S U(2)-\{I\}$, note que $\mathbb{T}$ não estabiliza

$$
i \lambda\left(\begin{array}{cc}
|\alpha|^{2}-|\beta|^{2} & 2 \alpha \bar{\beta} \\
2 \bar{\alpha} \beta & |\beta|^{2}-|\alpha|^{2}
\end{array}\right)
$$

de fato, para $h \in \mathbb{T}, h=\operatorname{diag}(x, \bar{x}), x \in U(1)$

$$
i \lambda h\left(\begin{array}{cc}
|\alpha|^{2}-|\beta|^{2} & 2 \alpha \bar{\beta} \\
2 \bar{\alpha} \beta & |\beta|^{2}-|\alpha|^{2}
\end{array}\right) h^{-1}=i \lambda\left(\begin{array}{cc}
|\alpha|^{2}-|\beta|^{2} & x^{2} 2 \alpha \bar{\beta} \\
\bar{x}^{2} 2 \bar{\alpha} \beta & |\beta|^{2}-|\alpha|^{2}
\end{array}\right)
$$

para que $h$ esteja no estabilizador deveríamos ter $x= \pm 1$, logo $\mathbb{T}$ não é o estabilizador, mas sabemos da Proposição 7.6 que o estabilizador é dado por $g \mathbb{T} g^{-1}$.

Para a ação coadjunta de $S U(2)$, existe apenas uma classe de conjugação dos subgrupos de isotropia, a saber, a classe $(\mathbb{T})$, onde 


$$
\mathbb{T}=\{g \in S U(2) \mid g=\operatorname{diag}(\alpha, \bar{\alpha}), \alpha \in U(1)\},
$$

a subvariedade com tipo de isotropia $\mathbb{T}, M_{\mathbb{T}}$, é dada por

$$
M_{\mathbb{T}}=\{X \in \mathfrak{s u}(2) \mid X=i \operatorname{diag}(\lambda,-\lambda), \lambda \in \mathbb{R} \backslash\{0\}\} \subset \mathfrak{s u}(2)
$$

devido a bijeção de $\mathfrak{s u}(2)$ com $\mathbb{R}^{3}$, temos que dado $X \in M_{\mathbb{T}}$ existe $v_{X} \in \mathbb{R}^{3}$ onde $v_{X}=(\lambda, 0,0)$, dessa forma, $\operatorname{dim} M_{\mathbb{T}}=1$.

Como pontos em uma mesma órbita estão em uma mesma subvariedade com tipo de órbita $(\mathbb{T})$, segue que dado $X \in M_{\mathbb{T}}$, e $\mathcal{O}_{X}$ a órbita passando por $X$, se $Y \in \mathcal{O}_{X}$ temos que $S U(2)_{Y}=g \mathbb{T} g^{-1}$ onde $g \in S U(2)$ é tal que $\operatorname{Ad}_{g} X=Y$, dessa forma $\mathcal{O}_{X} \subset M_{(\mathbb{T})}$.

Sabemos ainda que a ação de $S U(2)$ em $\mathfrak{s u}(2)$ não é livre, pois os elementos $\pm I$ estão no subgrupo de isotropia $S U(2)_{X}$ para todo $X \in \mathfrak{s u}(2)$.

Vamos agora determinar o normalizador $N(\mathbb{T})$ de $\mathbb{T}=S U(2)_{X}$ dado em (7.3) para $X \in \mathfrak{s u}(2)$, com $X=i \operatorname{diag}(\lambda,-\lambda), \lambda \neq 0$. Desde que

$$
N(\mathbb{T})=\left\{g \in S U(2) \mid g \mathbb{T} g^{-1}=\mathbb{T}\right\}
$$

segue que

$$
g h g^{-1}=h^{\prime}
$$

para $h, h^{\prime} \in \mathbb{T}$, assim

$$
\left(\begin{array}{cc}
a & -\bar{b} \\
b & \bar{a}
\end{array}\right)\left(\begin{array}{cc}
\alpha & 0 \\
0 & \bar{\alpha}
\end{array}\right)\left(\begin{array}{cc}
\bar{a} & \bar{b} \\
-b & a
\end{array}\right)=\left(\begin{array}{cc}
x & 0 \\
0 & \bar{x}
\end{array}\right), \alpha, x \in U(1)
$$

$\log 0$

$$
\left(\begin{array}{cc}
|a|^{2} \alpha+|b|^{2} \bar{\alpha} & a \bar{b}(\alpha-\bar{\alpha}) \\
\bar{a} b(\alpha-\bar{\alpha}) & |a|^{2} \bar{\alpha}+|b|^{2} \alpha
\end{array}\right)=\left(\begin{array}{cc}
x & 0 \\
0 & \bar{x}
\end{array}\right)
$$

da igualdade acima, temos que ter

$$
\left\{\begin{aligned}
|a|^{2} \alpha+|b|^{2} \bar{\alpha} & =x \\
a \bar{b}(\alpha-\bar{\alpha}) & =0
\end{aligned}\right.
$$

para este sistema temos três casos possíveis para determinar como deve ser os elementos de $N(\mathbb{T})$, são eles:

(i) Caso $a=0$, afim de que $g \in S U(2)$, temos que ter $b \neq 0$ e assim

$$
g=\left(\begin{array}{cc}
0 & -\bar{b} \\
b & 0
\end{array}\right), b \in U(1)
$$


(ii) Caso $b=0$, afim de que $g \in S U(2)$, temos que ter $a \neq 0$ e assim

$$
g=\left(\begin{array}{cc}
a & 0 \\
0 & \bar{a}
\end{array}\right), a \in U(1)
$$

(iii) Caso $a, b \neq 0$, teríamos uma restrição, a saber $\alpha=\bar{\alpha} \Rightarrow \alpha= \pm 1$, logo neste caso $g \neq N(\mathbb{T})$, pois satisfaz apenas para $\pm I \in \mathbb{T}$.

Dessa forma, concluímos que

$$
N(\mathbb{T})=\left\{g \in S U(2) \mid g=\left(\begin{array}{cc}
a & 0 \\
0 & \bar{a}
\end{array}\right) \text { ou } g=\left(\begin{array}{cc}
0 & -\bar{b} \\
b & 0
\end{array}\right)\right\},
$$

onde $a, b \in U(1)$, temos assim $\mathbb{T} \subset N(\mathbb{T}) \subset S U(2)$. Note que $N(\mathbb{T})=\mathbb{T} \cup \mathbb{T}^{\prime}$, onde $\mathbb{T}^{\prime}$ é dado por

$$
\mathbb{T}^{\prime}=\left\{g \in S U(2) \mid g=\left(\begin{array}{cc}
0 & -\bar{b} \\
b & 0
\end{array}\right), b \in U(1)\right\}=\left(\begin{array}{cc}
0 & 1 \\
-1 & 0
\end{array}\right) \cdot \mathbb{T}
$$

vale ressaltar que $\mathbb{T}$ é um grupo de Lie abeliano, unidimensional e compacto, enquanto que $\mathbb{T}^{\prime}$ claramente não é grupo, uma vez que nem tem o elemento identidade. Temos ainda que $N(\mathbb{T})$ é um grupo de Lie unidimensional, compacto com duas componentes conexas, $\mathbb{T} e \mathbb{T}^{\prime}$. O quociente $N(\mathbb{T}) / \mathbb{T}$ é o grupo de Weyl de $S U(2)$, o qual é um grupo com dois elementos com representantes em $N(\mathbb{T})$ dados por

$$
\left.e=\left(\begin{array}{ll}
1 & 0 \\
0 & 1
\end{array}\right) \text { el=( } \begin{array}{cc}
0 & 1 \\
-1 & 0
\end{array}\right)
$$

note que os elementos $-e$ e $-l$ pertencem as classes de e e l respectivamente, pois

$$
e \mathbb{T}=-e \mathbb{T} \text { e } l \mathbb{T}=-l \mathbb{T}
$$

assim,

$$
N(\mathbb{T}) / \mathbb{T}=L(\mathbb{T})=\{[e],[l]\} .
$$

Da Proposição 7.17 temos que a ação natural de $L(\mathbb{T})$ em $M_{\mathbb{T}}$ é uma ação de Poisson livre e própria.

Analisemos o que a ação de $L(\mathbb{T})$ faz em $M_{\mathbb{T}}$, temos que

$$
M_{\mathbb{T}}=\{X \in \mathfrak{s u}(2) \mid X=i \operatorname{diag}(\lambda,-\lambda), \lambda \in \mathbb{R} \backslash\{0\}\} \subset \mathfrak{s u}(2)
$$

fazendo a bijeção $\mathfrak{s u}(2)$ com $\mathbb{R}^{3}$, temos que $M_{\mathbb{T}}$ corresponde ao eixo $x$ excluindo a origem, o qual denotaremos por $O_{x} \backslash\{0\}$, dessa forma $M_{\mathbb{T}}$ é formado por duas 
componentes conexas. $A$ ação de $L(\mathbb{T})$ em $M_{\mathbb{T}}$ é dada por

$$
\begin{aligned}
\bar{\psi}: L(\mathbb{T}) \times M_{\mathbb{T}} & \longrightarrow M_{\mathbb{T}} \\
([g], X) & \longmapsto \bar{\psi}([g], X)=g X g^{-1}
\end{aligned}
$$

note inicialmente que $l^{-1}=-l$, dessa forma

$$
l X l^{-1}=\left(\begin{array}{cc}
0 & 1 \\
-1 & 0
\end{array}\right)\left(\begin{array}{cc}
i \lambda & 0 \\
0 & -i \lambda
\end{array}\right)\left(\begin{array}{cc}
0 & -1 \\
1 & 0
\end{array}\right)=\left(\begin{array}{cc}
-i \lambda & 0 \\
0 & i \lambda
\end{array}\right)
$$

$\operatorname{assim} X=i \operatorname{diag}(\lambda,-\lambda)$ o qual corresponde ao vetor $v_{X}=(\lambda, 0,0) \mathrm{em}$ $\mathbb{R}^{3}$ é levado pela ação acima para $Y=i \operatorname{diag}(-\lambda, \lambda)$ que corresponde ao vetor $-v_{X}=(-\lambda, 0,0)$ em $\mathbb{R}^{3}$, dessa forma, podemos identificar o quociente $M_{\mathbb{T}} / L(\mathbb{T})$ com a parte positiva do eixo $x$, que denotaremos por $O_{x>0}$

$$
M_{\mathbb{T}} / L(\mathbb{T}) \backsim O_{x>0} \simeq \mathbb{R}_{>0}
$$

já sabemos que este quociente é uma variedade de Poisson.

Para a ação adjunta de $S U(2)$ em $\mathfrak{s u}(2)$, existem dois subgrupos de isotropia em $S U(2)$ que geram as subvariedades com tipo de órbita $\left(S U(2)_{X}\right)$, os quais são $\mathbb{T}$ dado em (7.3) e o próprio $S U(2)$, mais precisamente, para qualquer $X \in \mathfrak{s u}(2)$ o estabilizador de $X$ é $S U(2)$ se $X=0$, e se $X \neq 0$ o estabilizador pertence a classe de conjugação de $\mathbb{T}$. Portanto existem apenas duas subvariedades com tipo de órbita $\left(S U(2)_{X}\right)$, a saber

$$
M_{(\mathbb{T})}=\mathfrak{s u}^{*}(2)_{(\mathbb{T})}=\mathfrak{s u}^{*}(2) \backslash\{0\} \text { e } M_{(S U(2))}=\mathfrak{s u}^{*}(2)_{(S U(2))}=\{0\},
$$

do item $(i)$ da Proposição 7.21, segue que $\mathfrak{s u}^{*}(2)_{(\mathbb{T})} / S U(2)$ herda uma estrutura de Poisson de $M_{\mathbb{T}} / L(\mathbb{T})$. Note que se $A \in \mathfrak{s u}^{*}(2)_{(\mathbb{T})} / S U(2)$ então $A=\mathbb{S}_{|\lambda|}^{2}$, onde $\lambda \in \mathbb{R}$ é tal que existe $X=i \operatorname{diag}(\lambda,-\lambda) \in \mathfrak{s u}^{*}(2)$, assim o quociente $\mathfrak{s u}^{*}(2)_{(\mathbb{T})} / S U(2)$ é formado por esferas centradas na origem, dessa forma, as componentes conexas de $\mathfrak{s u}^{*}(2)_{(\mathbb{T})} / S U(2)$ são essas esferas, e de acordo com o Teorema 7.22, essas esferas formam uma estratificação de Poisson do espaço de órbitas $\mathfrak{s u}^{*}(2) / S U(2)$, ou seja, $\left\{c_{j}\left(\mathfrak{s u}(2)_{(\mathbb{T})} / S U(2)\right)\right\}_{j \in I} \cup\{0\}$ é a estratificação, note que retornamos para o dual sem fazer menção, isto se deve a correspondência entre a álgebra de lie e o seu dual neste caso.

Agora já sabemos a estratificação do espaço de órbitas $\mathfrak{s u}^{*}(2) / S U(2)$, nos falta ainda determinar as folhas simpléticas das estratas, isto será possivel a partir do Teorema 7.40, o qual nos fornece a folheação das estratas desse espaço. 
Exemplo 7.26 Considere a ação coadjunta diagonal de $S U(2)$ na variedade $M=\prod_{j=1}^{n} \mathfrak{s u}^{*}(2)$, queremos descrever o quociente $M_{\mathbb{T}} / L(\mathbb{T})$, onde $\mathbb{T}$ é um subgrupo de isotropia. Seja $\mathbb{T}$ o subgrupo de isotropia dado em (7.3), no exemplo anterior determinamos o grupo $N(\mathbb{T})$ o qual está descrito em (7.4), a subvariedade $M_{\mathbb{T}}$ é por definição

$$
M_{\mathbb{T}}=\left\{X \in \prod_{j=1}^{n} \mathfrak{s u}^{*}(2) \mid S U(2)_{X}=\mathbb{T}\right\}
$$

notamos facilmente que $M_{\mathbb{T}}$ é formada por $n$ tipos de pontos, os quais são, a menos de uma permutação, da forma:

$$
\left\{\left\{X_{1}, \ldots, X_{n}\right\},\left\{X_{1}, \ldots, X_{n-1}, 0\right\}, \ldots,\left\{X_{1}, 0, \ldots, 0\right\}\right\}
$$

onde $X_{j}=i \operatorname{diag}\left(\lambda_{j},-\lambda_{j}\right), \lambda_{j} \neq 0$ para todo $j$, dessa forma

$$
M_{\mathbb{T}}=\left\{X \in M \mid X \neq 0, \text { se } X_{j} \neq 0 \text { então } X_{j}=i \operatorname{diag}\left(\lambda_{j},-\lambda_{j}\right)\right\}
$$

$\lambda_{j} \in \mathbb{R} \backslash\{0\}$, o grupo $L(\mathbb{T})$ já foi descrito em (7.5), no exemplo anterior fizemos a identificação

$$
\mathfrak{s u}^{*}(2)_{\mathbb{T}} \simeq \mathbb{R} \backslash\{0\}
$$

portanto, com o devido cuidado de identificação, obtemos:

$$
M_{\mathbb{T}}=\left(\prod_{j=1}^{n} \mathfrak{s u}^{*}(2)\right)_{H} \simeq \mathbb{R}^{n} \backslash\{0\}
$$

portanto,

$$
M_{\mathbb{T}} / L(\mathbb{T}) \simeq\left(\prod_{j=1}^{n} \mathbb{R}_{\geq 0}\right) \backslash\{0\}
$$

o qual é uma variedade de Poisson. Note que $M_{\mathbb{T}} / L(\mathbb{T})$ é identificado com os pontos de $\mathbb{R}^{n}$ com entradas não negativas, excluindo a origem.

Notamos que

$$
M_{\mathbb{T}}=\left(\prod_{j=1}^{n} \mathfrak{s u}^{*}(2)\right)_{\mathbb{T}} \supset \prod_{j=1}^{n}(\mathfrak{s u}(2))_{\mathbb{T}}=M_{\mathbb{T}}^{\prime},
$$

de fato, se $X \in M_{\mathbb{T}}^{\prime}$, então $X=\left(X_{1}, \ldots, X_{n}\right)$ é tal que $X_{j}=i \operatorname{diag}\left(\lambda_{j},-\lambda_{j}\right)$ para todo $j$, com $\lambda_{j} \neq 0$ para todo $j$, isto significa que $S U(2)_{X}=\mathbb{T}$, portanto, $X \in M_{\mathbb{T}}$, dai

$$
M_{\mathbb{T}}^{\prime} \subset M_{\mathbb{T}}
$$


A igualdade não ocorre, para ver isto, basta tomar $\xi=\left(\xi_{1}, \ldots, \xi_{n}\right) \in M_{\mathbb{T}}$, logo $\xi_{j}=i \operatorname{diag}\left(\lambda_{j}-\lambda_{j}\right)$ para todo $j$, note que não é necessário a restrição de $\lambda_{j} \neq 0$ para todo $j$, dessa forma, $\xi$ pode ter até $n-1$ entradas nulas, suponha sem perda de generalidade que a $k$-ésima entrada de $\xi$ é nula, mas isto significa que $S U(2)_{\xi_{k}}=S U(2)$, portanto $\xi_{k} \notin \mathfrak{s u}{ }^{*}(2)_{\mathbb{T}}$, logo $\xi \notin M_{\mathbb{T}}^{\prime}$, e assim $M_{\mathbb{T}} \neq M_{\mathbb{T}}^{\prime}$.

Agora, com relação a subvariedade com tipo de órbita $(\mathbb{T}), M_{(\mathbb{T})}$, sejam

$$
M_{(\mathbb{T})}=\left(\prod_{j=1}^{n} \mathfrak{s u}^{*}(2)\right)_{(\mathbb{T})} \text { e } M_{(\mathbb{T})}^{\prime}=\prod_{j=1}^{n}(\mathfrak{s u}(2))_{(\mathbb{T})}
$$

essas variedades claramente não são disjuntas, uma vez que $M_{\mathbb{T}}^{\prime} \subset M_{\mathbb{T}}$, apesar disso não ocorre as inclusões $M_{(\mathbb{T})} \subset M_{(\mathbb{T})}^{\prime}$ e $M_{(\mathbb{T})}^{\prime} \subset M_{(\mathbb{T})}$, de fato, por um lado em $M_{(\mathbb{T})}^{\prime}$ existem pontos com entradas que admitem diferentes estabilizadores em $(\mathbb{T})$, enquanto que em $M_{(\mathbb{T})}$ as entradas de todos os pontos podemos ser estabilizadas pelo mesmo subgrupo em $(\mathbb{T})$, portanto não ocorre $M_{(\mathbb{T})}^{\prime} \subset M_{(\mathbb{T})}$. Por outro lado, $M_{(\mathbb{T})} \subset M_{(\mathbb{T})}^{\prime}$ também não ocorre, uma vez que em $M_{(\mathbb{T})}$ existem pontos com entradas nulas, enquanto que em $M_{(\mathbb{T})}^{\prime}$ as entradas de todos os pontos são não nulas.

O quociente $M_{\mathbb{T}} / L(\mathbb{T})$ é Poisson e induz uma estrutura de Poisson em $M_{(\mathbb{T})} / S U(2)$, isto é garantido pelo item $(i)$ da Proposição 7.21. Afim de descreve este último quociente, notamos que em $M_{(\mathbb{T})}$ temos os pontos, a menos de uma permutação das entradas, da forma $X=\left(X_{1}, \ldots, X_{k}, 0, \ldots, 0\right)$, onde $X_{j}=i \operatorname{diag}\left(\lambda_{j},-\lambda_{j}\right), \lambda_{j} \in \mathbb{R} \backslash\{0\}, j=1, \ldots, k$, com $1 \leq k \leq n$, os quais são estabilizados por $\mathbb{T}$, estes pontos serão referidos como tendo forma diagonal, e além disso, para qualquer $Y \in M_{(\mathbb{T})}$ sempre existe um $X \in M_{\mathbb{T}} \subset M_{(\mathbb{T})} d a$ forma diagonal tal que $Y$ pode ser escrito como

$$
Y=\operatorname{Ad}_{g} X=\left(\operatorname{Ad}_{g} X_{1}, \ldots, \operatorname{Ad}_{g} X_{k}, 0 \ldots, 0\right)
$$

para algum $g \in S U(2)$, isto significa que $Y$ sempre pertence a alguma órbita coadjunta diagonal de $S U(2)$ passando por algum $X \in M_{\mathbb{T}}$ e essa órbita denotaremos por:

$$
\mathcal{O}_{X}^{\mathcal{D}} \simeq \mathcal{D}_{X}\left(\prod_{j=1}^{n} \mathbb{S}_{\left|\lambda_{j}\right|}^{2}\right) \subset \prod_{j=1}^{n} \mathbb{S}_{\left|\lambda_{j}\right|}^{2}
$$

lembrando que o raio da j-ésima esfera é dado pela norma do autovalor de $X_{j}$, no caso de $X_{j}=0$ a esfera reduz-se a um ponto, a origem. A inclusão acima é devido a ação ser diagonal, isto significa que para todo $Y \in \mathcal{O}_{X}^{\mathcal{D}}$ existe $g \in S U(2)$ tal que $\operatorname{Ad}_{g} X=Y$, enquanto que do lado direito existem pontos que não estão na órbita coadjunta diagonal de $S U(2)$ passando por qualquer $X$ da forma diagonal mencionada acima. O produto de esferas aparece na órbita 
$\mathcal{O}_{X}^{\mathcal{D}}$ pois a órbita coadjunta de $S U(2)$ passando por $X_{j}$ é a esfera $\mathbb{S}_{\left|\lambda_{j}\right|}^{2}$ para todo j. Pelo exposto, concluímos claramente que $\mathcal{O}_{X}^{\mathcal{D}} \subset M_{(\mathbb{T})}$, portanto, se $A \in M_{(\mathbb{T})} / S U(2)$ então $A$ é da forma $\mathcal{O}_{X}^{\mathcal{D}}$ para algum $X \in M_{\mathbb{T}}$. Sabemos que $S U(n)$ é um grupo de Lie conexo, logo $\mathcal{O}_{X}^{\mathcal{D}}$ é conexa e portanto c $\left(M_{(\mathbb{T})} / S U(2)\right)$ é dado pela órbita $\mathcal{O}_{X}^{\mathcal{D}}$ para algum $X \in M_{(\mathbb{T})}$.

Vale ressaltar que o ponto $\boldsymbol{O}=(0, \ldots, 0)$ não pertence a nenhuma subvariedade com tipo de órbita $(\mathbb{T})$, esse ponto é o único ponto de $M_{S U(2)}$ e de $M_{(S U(2))}$.

Resumidamente $c\left(M_{(\mathbb{T})} / S U(2)\right)=\mathcal{O}_{X}^{\mathcal{D}}=\mathcal{D}_{X}\left(\prod_{j=1}^{m} \mathbb{S}_{\left|\lambda_{j}\right|}^{2}\right)$ para algum $X \in \prod_{j=1}^{m} \mathfrak{s u}^{*}(2), \operatorname{assim}\left\{c_{j}\left(M_{(\mathbb{T})} / S U(2)\right)\right\}_{j \in I} \cup\{0\} \quad e ́$ a estratificação de $\left(\prod_{j=1}^{m} \mathfrak{s u}(2)\right) / S U(2)$.

Exemplo 7.27 Considere a variedade simplética $\mathcal{S}=\prod_{j=1}^{m} \mathbb{S}_{\left|\lambda_{j}\right|}^{2}$, sabemos que $\mathbb{S}_{\left|\lambda_{j}\right|}^{2} \simeq \mathbb{F}_{\mathbb{C}}^{j}(1,1) \simeq \mathcal{O}_{X_{j}}, X_{j} \in \mathfrak{s u}^{*}(2), X_{j}$ satisfaz a condição de que existe $g \in S U(2)$ tal que $g X_{j} g^{-1}=i \operatorname{diag}\left(\lambda_{j},-\lambda_{j}\right)$, onde $\lambda_{j}$ é o autovalor de $X_{j}$, ou seja, $\mathbb{S}_{\left|\lambda_{j}\right|}^{2}$ pode ser vista como uma órbita coadjunta de $S U(2)$ por $X_{j}$.

Agora considere a ação coadjunta diagonal de $S U(2)$ em $\mathcal{S}$

$$
\begin{aligned}
\psi: S U(2) \times \mathcal{S} & \longrightarrow \mathcal{S} \\
(g, \xi) & \longmapsto\left(\operatorname{Ad}_{g}^{*} \xi_{1}, \ldots, \operatorname{Ad}_{g}^{*} \xi_{m}\right)
\end{aligned}
$$

esta ação não é livre pois em $\mathcal{S}$ existe o ponto $\xi$ onde todas as entradas $\xi_{j}$ são da forma $\xi_{j}=i \operatorname{diag}\left(\lambda_{j},-\lambda_{j}\right)$, e assim, o estabilizador de $\xi$ é dado por $S U(2)_{\xi}=\mathbb{T}$, onde

$$
\mathbb{T}=\{g \in S U(2) \mid g=\operatorname{diag}(\alpha, \bar{\alpha}), \alpha \in U(1)\},
$$

considerando apenas uma entrada de $\xi$, temos que todo ponto da órbita de $S U(2)$ passando por $\xi_{j}$ é estabilizado por algum subgrupo conjugado a $\mathbb{T}$, ou seja, $g \mathbb{T} g^{-1}$ para algum $g \in S U(2)$. Portanto os subgrupos de isotropia desta ação pertencem a classe de conjugação de $\mathbb{T}$, vale ressaltar que o normalizador $N(\mathbb{T})$ e $L(\mathbb{T})$ forma determinados em $(7.4)$ e (7.5), respectivamente. Vejamos agora as subvariedades $\mathcal{S}_{\mathbb{T}}$ e $\mathcal{S}_{(\mathbb{T})}$

$$
\mathcal{S}_{\mathbb{T}}=\left\{\xi=\left(\xi_{1}, \ldots, \xi_{m}\right) \in \mathcal{S} \mid S U(2)_{\xi}=\mathbb{T}\right\}
$$

como a ação é a coadjunta diagonal, temos que ter $S U(2)_{\xi_{j}}=\mathbb{T}$ para todo 
$j, \operatorname{logo} \xi_{j}=i \operatorname{diag}\left(\lambda_{j},-\lambda_{j}\right)$ e assim o ponto $\xi$ mencionado acima é o único ponto em $\mathcal{S}_{\mathbb{T}}$. Note que a escolha da variedade $\mathcal{S}$ pode ser feita de tal forma que o vetor de comprimento $s=\left(\left|\lambda_{1}\right|, \ldots,\left|\lambda_{m}\right|\right)$ não tenha entradas nulas, $\operatorname{logo} \xi \in \mathcal{S}_{\mathbb{T}}$ não terá entradas nulas. Enquanto que a subvariedade com tipo de órbita $(\mathbb{T})$ é dada por

$$
\mathcal{S}_{(\mathbb{T})}=\left\{X \in \mathcal{S} \mid \operatorname{Ad}_{g}^{*} X=\xi \text { para algum } g \in S U(2)\right\}=\left\{X \in \mathcal{S} \mid X \in \mathcal{O}_{\xi}^{\mathcal{D}}\right\}
$$

onde $\xi \in \mathcal{S}_{\mathbb{T}}$, ou seja, $\mathcal{S}_{(\mathbb{T})} \simeq \mathcal{O}_{\xi}^{\mathcal{D}}$. Portanto se $A \in \mathcal{S}_{(\mathbb{T})} / S U(2)$ então A é tal que

$$
A=\mathcal{O}_{\xi}^{\mathcal{D}}=\mathcal{D}_{\xi}\left(\prod_{j=1}^{m} \mathbb{S}_{\left|\lambda_{j}\right|}^{2}\right)
$$

onde $\xi=\left(\xi_{1}, \ldots, \xi_{m}\right)$ e $\xi_{j}=i \operatorname{diag}\left(\lambda_{j},-\lambda_{j}\right)$ pra todo $j$, como $S U(2)$ é conexo, concluímos que A é uma estrata de $\mathcal{S} / S U(2)$.

O mapa momento associado a esta ação é dado por

$$
\begin{aligned}
\mu: \mathcal{S} & \longrightarrow \mathfrak{s u}^{*}(2) \\
\xi & \longmapsto \mu(\xi)=\sum_{j=1}^{m} \xi_{j}
\end{aligned}
$$

Note que $\mu^{-1}(0) \subset \mathcal{S}$ quocientado por $S U(2)$, descreve o espaço dos $m$-gons de lados de comprimentos fixados $\left|\lambda_{j}\right|$, onde $\mu^{-1}(0) / S U(2)$ é uma variedade suave se o vetor de comprimento $s=\left(\left|\lambda_{1}\right|, \ldots,\left|\lambda_{m}\right|\right)$ é genérico.

$E$ ainda, $\mu^{-1}(\eta) \subset \mathcal{S}, \eta \in \mathfrak{s u}^{*}(2)$, pode ser visto como o espaço dos $(m+1)$-gons em $\mathcal{S}$ de lados de comprimentos fixados $s=\left(\left|\lambda_{1}\right|, \ldots,\left|\lambda_{m}\right|, s_{\eta}\right)$ onde a última entrada do vetor de comprimento depende do nivel $\eta$ escolhido, $\|\eta\|=s_{\eta}$, note ainda que $S U(2)_{\eta} \in(\mathbb{T})$, pois $\eta$ pertence a órbita coadjunta dada pela esfera $\mathbb{S}_{|\eta|}^{2}$.

Exemplo 7.28 Com o intuito de apresentar em trabalhos futuro a estratificação de Poisson detalhada de $\mathfrak{s u}^{*}(3)$ e $\mathfrak{s u}^{*}(3) / S U(3)$, damos início a esse estudo apresentando algumas informações conhecidas.

Sendo $X \in \mathfrak{s u}(3)$, então $X$ é da forma descrita em (6.5), sabemos que existe $g \in S U(3)$ tal que

$$
\operatorname{Ad}_{g} X=i \operatorname{diag}\left(\lambda_{1}, \lambda_{2}, \lambda_{3}\right)
$$

Usando este fato da diagonalização, podemos facilmente chegar a conclusão que a ação coadjunta de $S U(3)$ não é livre e que admite três classes de subgrupos de isotropia, esta conclusão o prezado leitor pode chegar a partir da leitura cuidadosa do Exemplo 6.26, onde apresentamos as órbitas dessa ação. A partir 
dessas três classes podemos inferir as subvariedades com tipo de isotropia e com tipo de órbita.

Em [27] encontramos os estabilizadores da ação de $S U(3)$ em $\mathfrak{s u ( 3 ) ,}$ são eles: $S U(3), S(U(2) \times U(1))$ e $S(U(1) \times U(1) \times U(1))$, estes subgrupos estabilizam a origem, os pontos diagonais que possuem exatamente dois autovalores iguais e aqueles pontos diagonais que possuem os autovalores dois a dois distintos, respectivamente.

Agora que sabemos os estabilizadores, podemos determinar as subvariedades $\mathfrak{s u}^{*}(3)_{H}$ e $\mathfrak{s u}^{*}(3)_{(H)}$, onde $H$ é subgrupo de isotropia.

1. Estabilizador $S U(3)$.

Para este caso temos

$$
\mathfrak{s u}^{*}(3)_{S U(3)}=\{0\}=\mathfrak{s u}^{*}(3)_{(S U(3))}
$$

2. Estabilizador $S(U(2) \times U(1))$.

Para este caso temos

$$
\mathfrak{s u}^{*}(3)_{S(U(2) \times U(1))}=\left\{Y \in \mathfrak{s u}^{*}(3) \mid X=i \operatorname{diag}(\lambda, \lambda,-2 \lambda)\right\}
$$

onde $\lambda \in \mathbb{R}$, com $\lambda \neq 0$. Para este caso a subvariedade com tipo de órbita $(S(U(2) \times U(1)))$ é dada por

$$
\begin{aligned}
& \mathfrak{s u}^{*}(3)_{(S(U(2) \times U(1)))}=\left\{Y \in \mathfrak{s u}^{*}(3) \mid \exists g \in S U(3), \operatorname{Ad}_{g}^{*} Y=i \operatorname{diag}(\lambda, \lambda,-2 \lambda)\right\} \\
& \text { ou seja, todos os pontos das órbitas de } S U(3) \text { por pontos de } \\
& \mathfrak{s u}^{*}(3)_{S(U(2) \times U(1))} .
\end{aligned}
$$

3. Estabilizador $S(U(1) \times U(1) \times U(1))$.

Para este caso temos

$$
\mathfrak{s u}^{*}(3)_{S(U(1) \times U(1) \times U(1))}=\left\{Y \in \mathfrak{s u}^{*}(3) \mid Y=i \operatorname{diag}\left(\lambda_{1}, \lambda_{2}, \lambda_{3}\right)\right\}
$$

onde $\lambda_{1}, \lambda_{2}$ e $\lambda_{3}$ são dois a dois distintos. A subvariedade com tipo de órbita $(S(U(1) \times U(1) \times U(1)))$ é dada por

$$
\mathfrak{s u}^{*}(3)_{(S(U(1) \times U(1) \times U(1)))}=\left\{Y \in \mathfrak{s u}^{*}(3) \mid \exists g \in S U(3), \operatorname{Ad}_{g}^{*} X=i \operatorname{diag}\left(\lambda_{1}, \lambda_{2}, \lambda_{3}\right)\right\}
$$

onde $\lambda_{1}, \lambda_{2}$ e $\lambda_{3}$ são dois a dois distintos, ou seja, todos os pontos das órbitas de $S U(3)$ por pontos de $\mathfrak{s u}^{*}(3)_{S(U(1) \times U(1) \times U(1))}$. 
Afim de que se obtenha a estratificação de Poisson faz-se necessário a determinação dos subgrupos normalizadores $N(H)$ desses subgrupos, onde $H$ é um subgrupo de isotropia qualquer, posteriormente determina-se o grupo $L(H)=N(H) / H$. Sabemos que a ação do grupo $L(H)$ nas subvariedade com tipo de isotropia é livre e própria. A continuidade desta análise deixamos para um trabalho futuro, onde iremos estudar as folhas simpléticas das estratas.

\subsection{2}

Estratificação das variedades $\mathfrak{s u}^{*}(n), \prod_{j=1}^{m} \mathfrak{s u}^{*}(n)$

Façamos o mesmo agora para o grupo $S U(n)$, o ponto de partida é escolher um subgrupo de isotropia, uma vez que todo ponto de $\mathfrak{s u}^{*}(n)$ pode ser diagonalizado, os subgrupos de isotropia podem ser os seguintes:

1. O toro maximal em $S U(n)$ o qual nos dará informações a respeito dos pontos em $\mathfrak{s u}^{*}(n)$ que possuem $n$ autovalores dois a dois distintos;

2. Ou o subgrupo é formado por elementos que são diagonais por bloco da forma $G_{d}=S U(n) \cap E_{d}$, onde

$$
E_{d}=\left\{\operatorname{diag}\left(a_{1}, \ldots, a_{r}\right) \mid a_{j} \in U\left(d_{j}\right), d_{j} \in \mathbb{N}, j=1, \ldots, r\right\}
$$

onde $d=\left(d_{1}, \ldots, d_{r}\right)$, com $d_{1}+\cdots+d_{r}=n$ e $2 \leq r<n$. Este subgrupo nos dará informações a respeito dos pontos em $\mathfrak{s u}^{*}(n)$ que possuem $r$ autovalores dois a dois distintos com multiplicidades $d_{1}, \ldots, d_{r}$.

Veremos esses dois casos nos exemplos a seguir. O normalizador nesses casos pode ser visto da seguinte forma: Seja $\mathbb{K}$ o conjunto de todas as sequências das multiplicidades dos autovalores de $X \in \mathfrak{s u}^{*}(n), d=\left(d_{1}, \ldots, d_{r}\right)$ de comprimentos $r=1,2, \ldots$ de números inteiros positivos satisfazendo $d_{1}+$ $\cdots+d_{r}=n$. Considere $p \in S_{r}$ uma permutação de $r$ elementos. Para $p \in S_{r}$, defina $p_{d} \in S_{n}$ a permutação de $n$ elementos obtida dividindo $(1, \ldots, n)$ em $r$ subsequências $\left(1, \ldots, d_{1}\right),\left(d_{1}+1, \ldots, d_{1}+d_{2}\right), \ldots,\left(d_{1}+\cdots+d_{r-1}+1, \ldots, n\right)$ e permutando estas subsequências de acordo com $p$. Desde que $g \in N\left(G_{d}\right)$ se, e somente se, $\operatorname{Ad}_{g} M_{G_{d}} \subset M_{G_{d}}$, veja Proposição 7.14, o normalizador $N\left(G_{d}\right)$ é gerado por $G_{d}$ e as matrizes de permutação de $p_{d}$ para todo $p \in S_{r}$ satisfazendo $p(d)=d$, este comentário pode ver visto em [19], página 300 .

Observação 7.29 O comentário que segue é devido a equivalência das ações adjunta e coadjunta para o grupo $S U(n)$. Sabemos que se $X \in \mathfrak{s u}(n) \backslash\{0\}$, então $\operatorname{tr} X=0$, e que sempre existe $g \in S U(n)$ tal que

$$
g X g^{-1}=i \operatorname{diag}\left(\lambda_{1}, \ldots, \lambda_{n}\right)
$$


o qual pode ser reescrito, após uma permutação adequada p das entradas, da forma

$$
p\left(g X g^{-1}\right)=i \operatorname{diag}\left(\lambda_{1}^{\prime} I_{d_{1}}, \ldots, \lambda_{r}^{\prime} I_{d_{r}}\right)
$$

onde os inteiros positivos $d_{j}$ 's denotam as multiplicidades dos autovalores $\lambda_{j}$ 's, desde que $\operatorname{tr} X=0$ e $X \neq 0$, então os autovalores de $X$ não podem ser todos iguais, portanto

$$
1 \leq d_{j}<n, \text { para todo } j
$$

implicando que

$$
1<r<n, r \in \mathbb{N}
$$

Exemplo 7.30 Para o primeiro caso, considere a ação coadjunta de $S U(n)$ na variedade $\mathfrak{s u}^{*}(n)$, para esta ação, seja o toro maximal em $S U(n)$ dado por

$$
\mathbb{T}=\left\{t \in S U(n) \mid t=\operatorname{diag}\left(e^{i \theta_{1}}, \ldots, e^{i \theta_{n-1}}, e^{-i \sum_{1}^{n-1} \theta_{j}}\right), \theta_{j} \in \mathbb{R}\right\}
$$

o qual é um subgrupo de isotropia. A subvariedade com tipo de isotropia $\mathbb{T}$ é dada por

$$
\mathfrak{s u}^{*}(n)_{\mathbb{T}}=\left\{X \in \mathfrak{s u}^{*}(n) \mid S U(n)_{X}=\mathbb{T}\right\}
$$

note que $X$ satisfaz

$$
X=i \operatorname{diag}\left(\lambda_{1}, \ldots, \lambda_{n}\right), \sum_{j=1}^{n} \lambda_{j}=0, \text { e } \lambda_{i} \neq \lambda_{j} \text { se } i \neq j
$$

ou seja, $\mathfrak{s u}^{*}(n)_{\mathbb{T}}$ é formado pelos elementos diagonais de $\mathfrak{s u}^{*}(n)$ que possuem $n$ autovalores distintos. Claramente temos

$$
\mathfrak{s u}^{*}(n)_{\mathbb{T}} \subsetneq \mathbb{R}^{n-1} \backslash\{0\}
$$

esta inclusão é devida a identificação feita em (6.9).

É conhecido que $L(\mathbb{T})=N(\mathbb{T}) / \mathbb{T}$ é o grupo de permutações $S_{n}$, o qual age própria e livremente em $\mathfrak{s u}^{*}(n)_{\mathbb{T}}$, dessa forma $\mathfrak{s u}^{*}(n)_{\mathbb{T}} / S_{n}$ admite uma única estrutura de Poisson, lembrando que $\mathfrak{s u}^{*}(n)_{\mathbb{T}}$ é uma subvariedade Lie-Dirac de $\mathfrak{s u}^{*}(n)$.

Observação $7.31 \mathrm{Em} \mathbb{R}^{2}$ a permutação consiste em fazer uma reflexão com relação a reta $y=x$, daí o quociente de $\mathbb{R}^{2}$ pela permutação, nos fornece um semiplano. Em $\mathbb{R}^{3}$ as permutações consistem em fazer reflexão com relação aos planos $x=y$ e $y=z$, daí o quociente de $\mathbb{R}^{3}$ pela permutação, nos fornece uma interseção de dois semiespaços. Prosseguindo com esse raciocínio, em $\mathbb{R}^{n-1}$ as 
permutações consistem em fazer reflexão com relação a hiperplanos, sejam

$$
\mathcal{H}_{j}=\left\{\left(x_{1}, \ldots, x_{n-1}\right) \in \mathbb{R}^{n-1} \mid\left\langle\left(a_{1}^{j}, \ldots, a_{n-1}^{j}\right),\left(x_{1}, \ldots, x_{n-1}\right)\right\rangle=0\right\}
$$

esses hiperplanos, onde $\left(a_{1}^{j}, \ldots, a_{n-1}^{j}\right)$ é o vetor ortogonal a $\mathcal{H}_{j}$, para cada hiperplano, tome o seguinte semiespaço

$$
\mathcal{S H}_{j}=\left\{\left(x_{1}, \ldots, x_{n-1}\right) \in \mathbb{R}^{n-1} \mid\left\langle\left(a_{1}^{j}, \ldots, a_{n-1}^{j}\right),\left(x_{1}, \ldots, x_{n-1}\right)\right\rangle \geq 0\right\}
$$

seguindo esse raciocínio, obtemos que o quociente

$$
\mathfrak{s u}^{*}(n)_{\mathbb{T}} / S_{n} \subsetneq \bigcap_{j=1}^{n-2} \mathcal{S} \mathcal{H}_{j}=c
$$

a inclusão estrita se dá devido o fato de que $0 \neq \mathfrak{s u}^{*}(n)_{\mathbb{T}}$

Como a subvariedade com tipo de órbita $(\mathbb{T})$ é dada por

$$
\mathfrak{s u}^{*}(n)_{(\mathbb{T})}=\left\{X \in \mathfrak{s u}^{*}(n) \mid S U(n)_{X} \in(\mathbb{T})\right\}
$$

ou seja, $X \in \mathfrak{s u}^{*}(n)_{(\mathbb{T})}$ se existe $g \in S U(n)$ tal que $S U(n)_{X}=g \mathbb{T} g^{-1}$ isto nos diz que $\mathfrak{s u}^{*}(n)_{(\mathbb{T})}$ é composta pelos pontos de $\mathfrak{s u}^{*}(n)$ tais que os autovalores são todos de multiplicidade 1 , ou seja,

$$
\mathfrak{s u}^{*}(n)_{(\mathbb{T})}=\left\{X \in \mathfrak{s u}^{*}(n) \mid g^{-1} X g=i \operatorname{diag}\left(\lambda_{1}, \ldots, \lambda_{n}\right), \quad \lambda_{i} \neq \lambda_{j} \text { se } i \neq j\right\}
$$

uma vez que $X \in \mathfrak{s u}^{*}(n)$ segue que $\lambda_{1}+\cdots+\lambda_{n}=0$.

Vale ressaltar que se $X \in \mathfrak{s u}^{*}(n)_{\mathbb{T}}$, a órbita coadjunta de $S U(n)$ por $X$ é $\mathcal{O}_{X} \simeq \mathbb{F}_{\mathbb{C}}(1, \ldots, 1) \subset \mathfrak{s u}^{*}(n)_{(\mathbb{T})}$, e ainda, para todo $Y \in \mathfrak{s u}^{*}(n)_{(\mathbb{T})}$ existe $X \in \mathfrak{s u}^{*}(n)_{\mathbb{T}}$ tal que $Y \in \mathcal{O}_{X}$, portanto $\mathfrak{s u}^{*}(n)_{(\mathbb{T})} / S U(n)$ é composto pelas órbitas coadjuntas que passam pelos elementos de $\mathfrak{s u}^{*}(n)_{\mathbb{T}}$, ou seja, se $A \in \mathfrak{s u}^{*}(n)_{(\mathbb{T})} / S U(n)$, então $A \simeq \mathbb{F}_{\mathbb{C}}(1, \ldots, 1)$. Sendo $S U(n)$ conexo, segue que $c\left(\mathfrak{s u} *(n)_{(\mathbb{T})} / S U(n)\right)=\mathbb{F}_{\mathbb{C}}(1, \ldots, 1)$.

Exemplo 7.32 Para o segundo caso, considere a ação coadjunta de $S U(n)$ na variedade $\mathfrak{s u}^{*}(n)$, para esta ação, seja $G_{d}=S U(n) \cap E_{d}$ um subgrupo de isotropia, onde

$$
E_{d}=\left\{\operatorname{diag}\left(a_{1}, \ldots, a_{r}\right) \mid a_{j} \in U\left(d_{j}\right), j=1, \ldots, r\right\}
$$

$d=\left(d_{1}, \ldots, d_{r}\right), d_{1}+\cdots+d_{r}=n$. A subvariedade com tipo de isotropia $G_{d}$ é formada por pontos de $\mathfrak{s u}^{*}(n)$ que são diagonais por bloco, esses pontos possuem exatamente $r$ autovalores com multiplicidades $d_{1}, \ldots, d_{r}$, dai essa subvariedade 
ser descrita por

$$
\mathfrak{s u}^{*}(n)_{G_{d}}=\left\{X \in \mathfrak{s u}^{*}(n) \mid X=i \operatorname{diag}\left(\lambda_{1} I_{d_{1}}, \ldots, \lambda_{r} I_{d_{r}}\right), \lambda_{i} \neq \lambda_{j} \text { se } i \neq j\right\}
$$

$\lambda_{j} \in \mathbb{R}$, os autovalores $\lambda_{j}$ 's satisfazem a condição $\lambda_{1} d_{1}+\cdots+\lambda_{r} d_{r}=0$. Vale ressaltar que nem todos os pontos diagonais de $\mathfrak{s u}^{*}(n)$ que possuem $r$ autovalores com multiplicidades $d_{1}, \ldots, d_{r}$ estão em $\mathfrak{s u}^{*}(n)_{G_{d}}$, pois ao fixar o subgrupo de isotropia $G_{d}$, fixamos também uma ordem das multiplicidades dos autovalores, ou seja, se $X \in \mathfrak{s u}^{*}(n)_{G_{d}}$ então necessariamente $X$ é da forma $X=i \operatorname{diag}\left(\lambda_{1} I_{d_{1}}, \ldots, \lambda_{r} I_{d_{r}}\right)$, portanto qualquer ponto $Y \in \mathfrak{s u}^{*}(n)$ com $r$ autovalores, que tenha a sequência das multiplicidades de seus autovalores distinta da sequência d por uma permutação, então $Y \notin \mathfrak{s u}^{*}(n)_{G_{d}}$, no entanto, $Y$ pertence a órbita coadjunta de $S U(n)$ que passa por algum ponto de $\mathfrak{s u}^{*}(n)_{G_{d}}$, logo $Y \in \mathfrak{s u}^{*}(n)_{\left(G_{d}\right)}$. Como a subvariedade com tipo de órbita $\left(G_{d}\right)$ é dada por

$$
\mathfrak{s u}^{*}(n)_{\left(G_{d}\right)}=\left\{X \in \mathfrak{s u}^{*}(n) \mid S U(n)_{X} \in\left(G_{d}\right)\right\}
$$

isto nos diz que $\mathfrak{s u}^{*}(n)_{\left(G_{d}\right)}$ é composta por todos os pontos de $\mathfrak{s u}^{*}(n)$ com exatamente $r$ autovalores distintos e tais que os autovalores são de multiplicidades $d_{1}, \ldots, d_{r}$, ou seja,

$$
\mathfrak{s u}^{*}(n)_{\left(G_{d}\right)}=\left\{X \in \mathfrak{s u}(n) \mid g^{-1} X g=i \operatorname{diag}\left(\lambda_{1} I_{d_{1}}, \ldots, \lambda_{r} I_{d_{r}}\right), g \in S U(n)\right\},
$$

vale ressaltar que o $g \in S U(n)$, depende de $X$, esta última se justifica da seguinte forma: Se $X \in \mathfrak{s u}^{*}(n)_{\left(G_{d}\right)}$ então existe $g \in S U(n)$ tal que $S U(n)_{X}=g G_{d} g^{-1}$, isto significa que $X \in \mathcal{O}_{\xi}$, para algum $\xi \in \mathfrak{s u}^{*}(n)_{G_{d}}$, ou seja, $X$ e $\xi$ possuem os mesmos autovalores e as mesmas multiplicidades desses autovalores. O fato de $X \in \mathcal{O}_{\xi}$ nos diz que existe $g \in S U(n)$ tal que

$$
\operatorname{Ad}_{g}^{*} \xi=X \Rightarrow g \xi g^{-1}=X \Rightarrow \xi=g^{-1} X g
$$

Vale ressaltar que se $X \in \mathfrak{s u}^{*}(n)_{G_{d}}$, então $\mathcal{O}_{X} \simeq \mathbb{F}_{\mathbb{C}}\left(d_{1}, \ldots, d_{r}\right) \subset \mathfrak{s u}^{*}(n)_{\left(G_{d}\right)}$, assim $\mathfrak{s u}^{*}(n)_{\left(G_{d}\right)} / S U(n)$ é composto pelas órbitas coadjuntas de $S U(n)$ que passam pelos elementos de $\mathfrak{s u}^{*}(n)_{G_{d}}$, ou seja, se $A \in \mathfrak{s u}^{*}(n)_{\left(G_{d}\right)} / S U(n)$, então $A \simeq \mathbb{F}_{\mathbb{C}}\left(d_{1}, \ldots, d_{r}\right)$. Sendo $S U(n)$ conexo, segue que $c\left(\mathfrak{s u}^{*}(n)_{(\mathbb{T})} / S U(n)\right)=$ $\mathbb{F}_{\mathbb{C}}\left(d_{1}, \ldots, d_{r}\right)$.

Vale ressaltar que o ponto $\boldsymbol{O} \in \mathfrak{s u}^{*}(n)$ não pertence a nenhuma subvariedade com tipo de órbita $(\mathbb{T})$ e nem com tipo de órbita $\left(G_{d}\right)$, esse ponto é o único ponto de $\mathfrak{s u}^{*}(n)_{S U(n)}$ e de $\mathfrak{s u}^{*}(n)_{(S U(n))}$.

Analisaremos agora a ação coadjunta diagonal de $S U(n)$ em $M=$ 
$\prod_{j=1}^{m} \mathfrak{s u}^{*}(n)$, assim como nos exemplos anteriores serão utilizados os dois tipos de subgrupos, a saber, $\mathbb{T}$ e $G_{d}$.

Observação 7.33 Sabemos que a órbita coadjunta de $S U(n)$ por $\xi_{j} \in \mathfrak{s u}(n)$ é dada pela variedade bandeira $\mathbb{F}_{\mathbb{C}}^{j}\left(d_{1}^{j}, \ldots, d_{r_{j}}^{j}\right)$, onde $\xi_{j}$ possui uma quantidade $r_{j}$ de autovalores distintos, com multiplicidades dada pela sequência $d^{j}=$ $\left(d_{1}^{j}, \ldots, d_{r_{j}}^{j}\right)$, assim podemos escrever a órbita coadjunta diagonal de $S U(n)$ por $\xi$, a qual será denotada por $\mathcal{O}_{\xi}^{\mathcal{D}}$ onde $\xi \in \prod_{j=1}^{m} \mathfrak{s u}(n)$, como um produto diagonal de variedades bandeiras, afim de diferenciar a notação para que não haja confusão de quais pontos estão na órbita, esse produto receberá um identificador $\mathcal{D}_{\xi}$

$$
\mathcal{O}_{\xi}^{\mathcal{D}}=\mathcal{D}_{\xi}\left(\prod_{j=1}^{m} \mathbb{F}_{\mathbb{C}}^{j}\left(d_{1}^{j}, \ldots, d_{r_{j}}^{j}\right)\right)
$$

o que está sendo enfatizado aqui é a órbita de uma ação diagonal, o identificador $\mathcal{D}$ indica que para quaisquer $\xi^{\prime}, \xi^{\prime \prime} \in \mathcal{O}_{\xi}^{\mathcal{D}}$, onde $\xi^{\prime}=\left(\xi_{1}^{\prime}, \ldots, \xi_{m}^{\prime}\right)$ e $\xi^{\prime \prime}=\left(\xi_{1}^{\prime \prime}, \ldots, \xi_{m}^{\prime \prime}\right)$ temos que sempre existe $g \in S U(n)$ tal que $\operatorname{Ad}_{g}^{*} \xi_{j}^{\prime}=\xi_{j}^{\prime \prime}$ para todo $j$, enquanto que o indice $\xi$, indica que a órbita contém o ponto $\xi$, assim conseguimos distinguir as órbitas que passam por dois pontos que possuem a mesma quantidade de autovalores distintos e a mesma sequência de multiplicidade desses autovalores, mas que estão em órbitas coadjuntas diagonais disjuntas, por exemplo, sejam

$$
X, Y \in \prod_{j=1}^{m} \mathbb{F}_{\mathbb{C}}^{j}\left(d_{1}^{j}, \ldots, d_{r_{j}}^{j}\right)
$$

nas condições mencionadas, ou seja, não existe $g \in S U(n)$ tal que $g \cdot X=Y$, dessa forma $\mathcal{O}_{X}^{\mathcal{D}} \cap \mathcal{O}_{Y}^{\mathcal{D}}=\emptyset$, isto é possivel da seguinte forma, basta que exista ao menos um autovalor de $X$ que não é autovalor de $Y$, assim as órbitas que passam por $X$ e $Y$ são distintas e dadas por

$$
\mathcal{O}_{X}^{\mathcal{D}}=\mathcal{D}_{X}\left(\prod_{j=1}^{m} \mathbb{F}_{\mathbb{C}}^{j}\left(d_{1}^{j}, \ldots, d_{r_{j}}^{j}\right)\right) e \mathcal{O}_{Y}^{\mathcal{D}}=\mathcal{D}_{Y}\left(\prod_{j=1}^{m} \mathbb{F}_{\mathbb{C}}^{j}\left(d_{1}^{j}, \ldots, d_{r_{j}}^{j}\right)\right)
$$

ficando assim claro a necessidade do índice na notação.

Feito esta observação, vamos agora para os exemplos.

Exemplo 7.34 Para a ação coadjunta diagonal de $S U(n)$ em $M=\prod_{j=1}^{m} \mathfrak{s u}^{*}(n)$, 
considere o toro maximal em $S U(n)$ dado por

$$
\mathbb{T}=\left\{t \in S U(n) \mid t=\operatorname{diag}\left(e^{i \theta_{1}}, \ldots, e^{i \theta_{n-1}}, e^{-i \sum_{1}^{n-1} \theta_{j}}\right), \theta_{j} \in \mathbb{R}\right\}
$$

o qual é um subgrupo de isotropia para a ação. Quando trabalhamos com a variedade $\mathfrak{s u}^{*}(n)$, mostramos que

$$
\mathfrak{s u}^{*}(n)_{\mathbb{T}}=\left\{\xi \in \mathfrak{s u}^{*}(n) \mid \xi=i \operatorname{diag}\left(\lambda_{1}, \ldots, \lambda_{n}\right), \sum_{j=1}^{n} \lambda_{j}=0, \quad \lambda_{i} \neq \lambda_{j} \text { se } i \neq j\right\}
$$

dessa forma podemos exibir as características dos pontos da subvariedade com tipo de isotropia $\mathbb{T}$, assim, se $X \in M_{\mathbb{T}}$, então $X=\left(X_{1}, \ldots, X_{m}\right)$ onde $X_{j}=i \operatorname{diag}\left(\lambda_{1}^{j}, \ldots, \lambda_{n}^{j}\right)$ de tal forma $q u e \sum_{k=1}^{n} \lambda_{k}^{j}=0$ para todo $j$, e ainda, caso $X_{j} \neq 0$, então $\lambda_{r}^{j} \neq \lambda_{s}^{j}$ se $r \neq s$ para todo $j$. Vale reforçar que $S U(n)_{X}=\mathbb{T}$ não implica $X_{j} \neq 0$ para todo $j$, dessa forma, $X$ pode ter entradas nula, evidentemente essa nulidade não pode ocorrer em todas as entradas, pois se assim fosse teríamos $S U(n)_{X}=S U(n)$. Note que os pontos $\xi \in M$ que possuem entradas nulas, têm no máximo $n-1$ entradas nulas, as demais são da forma diagonal. Portanto

$$
M_{\mathbb{T}}=\left\{\xi \in M \mid \xi_{j}=i \operatorname{diag}\left(\lambda_{1}^{j}, \ldots, \lambda_{n}^{j}\right), \lambda_{l}^{j} \neq \lambda_{k}^{j} \text { se } l \neq k\right\} \cup M_{\mathbb{T}}^{0}
$$

onde $M_{\mathbb{T}}^{0}$ é formado pelos pontos de $M$ que tem pelo menos uma entrada nula e no máximo $(n-1)$ entradas nulas e as $j$-ésimas entradas não nulas satisfazem

$$
\xi_{j}=i \operatorname{diag}\left(\lambda_{1}^{j}, \ldots, \lambda_{n}^{j}\right), \lambda_{l}^{j} \neq \lambda_{k}^{j} \text { se } l \neq k
$$

Usando argumento análogo ao do Exemplo 7.26, chegamos que

$$
\left(\prod_{j=1}^{m} \mathfrak{s u}^{*}(n)\right)_{\mathbb{T}} \supset \prod_{j=1}^{m}\left(\mathfrak{s u} \mathfrak{u}^{*}(n)\right)_{\mathbb{T}}
$$

$\dot{E}$ conhecido que $L(\mathbb{T})=N(\mathbb{T}) / \mathbb{T}$ é o grupo de permutações $S_{n}$, o qual age própria e livremente em $M_{\mathbb{T}}$, dessa forma $M_{\mathbb{T}} / S_{n}$ admite uma única estrutura de Poisson, uma vez que $M_{\mathbb{T}}$ é uma subvariedade Lie-Dirac de M. Assim,

$$
M_{\mathbb{T}} / L(\mathbb{T})=M_{\mathbb{T}} / S_{n} \subsetneq \prod_{j=1}^{m} c_{j}
$$

onde $c_{j}$ é a região descrita em (7.7) da Observação 7.31. Assim como no Exemplo 7.26, temos a inclusão dada em (7.9), mas para as variedades com 
tipo de órbita $(\mathbb{T})$ a seguir

$$
M_{(\mathbb{T})}=\left(\prod_{j=1}^{m} \mathfrak{s u}^{*}(n)\right)_{(\mathbb{T})} e M_{(\mathbb{T})}^{\prime}=\prod_{j=1}^{m}\left(\mathfrak{s u} \mathcal{u}^{*}(n)\right)_{(\mathbb{T})}
$$

não ocorre $M_{(\mathbb{T})} \subset M_{(\mathbb{T})}^{\prime}$ e nem $M_{(\mathbb{T})}^{\prime} \subset M_{(\mathbb{T})}$. Note que para todo $j, M_{(\mathbb{T})}$ é dado por

$$
\left\{\xi \in M \mid g^{-1} \xi_{j} g=i \operatorname{diag}\left(\lambda_{1}^{j}, \ldots, \lambda_{n}^{j}\right), \lambda_{l}^{j} \neq \lambda_{k}^{j} \text { se } l \neq k\right\} \cup M_{(\mathbb{T})}^{0}
$$

onde $M_{(\mathbb{T})}^{0}$ é o conjunto dos pontos que possuem de uma a no máximo $(n-1)$ entradas nulas e tais que para toda $j$-ésima entrada não nula exista $g \in S U(n)$ tal que

$$
g^{-1} \xi_{j} g=i \operatorname{diag}\left(\lambda_{1}^{j}, \ldots, \lambda_{n}^{j}\right), \lambda_{l}^{j} \neq \lambda_{k}^{j} \text { se } l \neq k
$$

enquanto que

$$
\prod_{j=1}^{m} \mathfrak{s u}^{*}(n)_{(\mathbb{T})}=\left\{\xi \in M \mid g_{j}^{-1} \xi_{j} g_{j}=i \operatorname{diag}\left(\lambda_{1}^{j}, \ldots, \lambda_{n}^{j}\right), \lambda_{l}^{j} \neq \lambda_{k}^{j} \text { se } l \neq k\right\}
$$

ou seja, enquanto que em (7.10) é o mesmo $g \in S U(n)$ para todo $j$, neste último para cada $j$ tem-se um $g_{j} \in S U(n)$ específico.

Vejamos agora uma distinção com relação ao Exemplo 7.26, a igualdade que ocorre naquele exemplo não acontece neste, ou seja, não temos

$$
\prod_{j=1}^{m}\left(\mathfrak{s u} \mathfrak{u}^{*}(n)\right)_{(\mathbb{T})}=\prod_{j=1}^{m} \mathfrak{s u}^{*}(n) \backslash\{0\}
$$

isto se deve ao fato de que do lado direito as entradas de cada ponto não tem necessariamente $n$ autovalores dois a dois distintos, enquanto que do lado esquerdo, todos os pontos têm essa característica para todas as suas entradas. Portanto o que ocorre é apenas a inclusão estrita

$$
\prod_{j=1}^{m}\left(\mathfrak{s u}^{*}(n)\right)_{(\mathbb{T})} \subsetneq \prod_{j=1}^{m} \mathfrak{s u}^{*}(n) \backslash\{0\}
$$

Sendo $\xi \in M_{\mathbb{T}}$ temos que a órbita coadjunta diagonal de $S U(n)$ por $\xi$ satisfaz a inclusão $\mathcal{O}_{\xi}^{\mathcal{D}} \subset M_{(\mathbb{T})}$, dessa forma, se $A \in M_{(\mathbb{T})} / S U(n)$ então

$$
A=\mathcal{O}_{\xi}^{\mathcal{D}} \simeq \mathcal{D}_{\xi}\left(\prod_{j=1}^{m} \mathbb{F}_{\mathbb{C}}^{j}(1, \ldots, 1)\right)
$$

para algum $\xi \in M_{\mathbb{T}}$, pois $\mathcal{O}_{\xi_{j}} \simeq \mathbb{F}_{\mathbb{C}}^{j}(1, \ldots, 1)$ para todo $j$. 
Vale ressaltar que o ponto $\boldsymbol{O}=(0, \ldots, 0) \in M$ não pertence a nenhuma subvariedade com tipo de órbita $(\mathbb{T})$, esse ponto é o único ponto de $M_{S U(n)}$ e de $M_{(S U(n))}$.

Exemplo 7.35 Considere o subgrupo de isotropia $G_{d}=S U(n) \cap E_{d}$, onde

$$
E_{d}=\left\{\operatorname{diag}\left(a_{1}, \ldots, a_{r}\right) \mid a_{j} \in U\left(d_{j}\right), j=1, \ldots, r\right\}
$$

com $r<n$, o caso $r=n$ é o exemplo anterior, ou seja, o caso onde $G_{d}=\mathbb{T}$, neste exemplo abordaremos o caso $r<n, G_{d}$ é subgrupo de isotropia para a ação coadjunta diagonal de $S U(n)$ em $M=\prod_{j=1}^{m} \mathfrak{s u}^{*}(n)$. Do Exemplo 7.32 sabemos que

$$
\mathfrak{s u}^{*}(n)_{G_{d}}=\left\{\xi \in \mathfrak{s u}^{*}(n) \mid \xi=i \operatorname{diag}\left(\lambda_{1} I_{d_{1}}, \ldots, \lambda_{r} I_{d_{r}}\right), \lambda_{j} \in \mathbb{R}\right\}
$$

onde $\lambda_{i} \neq \lambda_{j}$ se $i \neq j$ e com a condição de que $d_{1} \lambda_{1}+\ldots+d_{r} \lambda_{r}=0$, $d=\left(d_{1}, \ldots, d_{r}\right)$, usando isto poderemos ver como são os pontos da subvariedade com tipo de isotropia $G_{d}$, a qual por definição é dada por

$$
M_{G_{d}}=\left\{\xi \in M \mid S U(n)_{\xi}=G_{d}\right\}
$$

sendo $\xi \in M, \xi=\left(\xi_{1}, \ldots \xi_{m}\right), \xi_{j} \in \mathfrak{s u}^{*}(n)$ para todo $j$, se $\xi \in M_{G_{d}}$ então $S U(n)_{\xi_{j}}=G_{d}$ ou $\xi_{j}=0$, pois $G_{d}$ fixa todas as entradas e $\xi$, isto nos diz que as entradas dos pontos de $M_{G_{d}}$ ou são diagonais por blocos, ou são nulas, evidentemente para todo $\xi \in M_{G_{d}}$ pelo menos uma das entradas é não nula, pois caso contrário teríamos $S U(n)_{\xi}=S U(n)$. Resumidamente, se $\xi \in M_{G_{d}}$ então as entradas de $\xi$ podem ser

$$
\xi_{k}=0,0 \leq k<n \text { e } \xi_{j}=i \operatorname{diag}\left(\lambda_{1}^{j} I_{d_{1}}, \ldots, \lambda_{r}^{j} I_{d_{r}}\right), 0<j \leq n
$$

com $\lambda_{s}^{j} \in \mathbb{R}$ para todo $s$, de tal forma que $\lambda_{l}^{j} \neq \lambda_{s}^{j}$ se $l \neq s$ e satisfaz a condição $d_{1} \lambda_{1}^{j}+\cdots+d_{r} \lambda_{r}^{j}=0$ para todo $j$.

Usando argumento análogo ao utilizado no Exemplo 7.26, obtemos

$$
M_{G_{d}}=\left(\prod_{j=1}^{m} \mathfrak{s u}(n)\right)_{G_{d}} \supset \prod_{j=1}^{m}(\mathfrak{s u}(n))_{G_{d}} .
$$

A subvariedade com tipo de órbita $\left(G_{d}\right)$ é por definição

$$
M_{\left(G_{d}\right)}=\left\{\xi \in M \mid S U(n)_{\xi} \in\left(G_{d}\right)\right\}
$$

isto significa, que para cada ponto $\xi \in M_{\left(G_{d}\right)}$ existe $g \in S U(n)$ tal que 
$S U(n)_{\xi}=g G_{d} g^{-1}$, ou seja, se $\xi \in M_{\left(G_{d}\right)}, \xi=\left(\xi_{1}, \ldots \xi_{m}\right)$, existe $g \in S U(n)$ tal que

$$
g^{-1} \xi_{j} g=i \operatorname{diag}\left(\lambda_{1}^{j} I_{d_{1}}, \ldots, \lambda_{r}^{j} I_{d_{r}}\right)
$$

para todo $j$ onde $\xi_{j} \neq 0$, logo $\xi \in \mathcal{O}_{Y}^{\mathcal{D}}$ onde $Y=\left(Y_{1}, \ldots, Y_{m}\right) \in M_{G_{d}}$ com

$$
Y_{j}=i \operatorname{diag}\left(\lambda_{1}^{j} I_{d_{1}}, \ldots \lambda_{r}^{j} I_{d_{r}}\right)
$$

para todo $j$. Isto nos diz que para todo $\xi \in M_{\left(G_{d}\right)}$ existe $Y \in M_{G_{d}}$ tal que $\xi \in \mathcal{O}_{Y}^{\mathcal{D}}$, claramente $\mathcal{O}_{Y}^{\mathcal{D}} \subset M_{\left(G_{d}\right)}$. Portanto, se $A \in M_{\left(G_{d}\right)} / S U(n)$, então

$$
A=\mathcal{O}_{Y}^{\mathcal{D}} \backsim \mathcal{D}_{Y}\left(\prod_{j=1}^{m} \mathbb{F}_{\mathbb{C}}^{j}\left(d_{1}, \ldots, d_{r}\right)\right)
$$

para algum $Y \in M_{G_{d}}$, uma vez que $\mathcal{O}_{Y_{j}} \simeq \mathbb{F}_{\mathbb{C}}^{j}\left(d_{1}, \ldots, d_{r}\right)$ e a ação é diagonal.

Vale ressaltar que o ponto $\boldsymbol{O}=(0, \ldots, 0) \in M$ não pertence a nenhuma subvariedade com tipo de órbita $\left(G_{d}\right)$, esse ponto é o único ponto de $M_{G_{d}}$ e de $M_{\left(G_{d}\right)}$.

\section{2}

\section{Folhas simpléticas das estratas}

Nesta seção enunciaremos alguns resultados referente a folheação do espaço $M / G$, onde $M$ é uma variedade de Poisson e $G$ um grupo de Lie que age propriamente em $M$. Para maiores detalhes, esses resultados podem ser encontrados em [3] e [10].

Sejam $(M,\{\cdot, \cdot\})$ uma variedade de Poisson e $G$ um grupo de Lie cuja ação em $M$ é uma ação de Poisson própria

$$
\psi: G \times M \rightarrow M
$$

denotamos por $A_{G}$ o grupo das transformações de Poisson associado a ação

$$
A_{G}:=\left\{\psi_{g}: M \rightarrow M \mid g \in G\right\}
$$

e $A_{G}^{\prime}$ a distribuição generalizada integrável definida por

$$
A_{G}^{\prime}(m):=\left\{X_{f}(m) \mid f \in \mathcal{C}^{\infty}(M)^{G}\right\}, \forall m \in M
$$

O mapa momento ótimo $J$ é definido como a projeção canônica no espaço de folhas de $A_{G}^{\prime}$

$$
J: M \rightarrow M / A_{G}^{\prime}
$$


Proposição 7.36 Sejam $(M,\{\cdot, \cdot\})$ uma variedade de Poisson e G um grupo de Lie que age em $M$ com uma ação de Poisson própria. Seja $J: M \rightarrow M / A_{G}^{\prime}$ o mapa momento ótimo. Então para qualquer $\rho \in M / A_{G}^{\prime}$ temos que:

(i) O conjunto de nível $J^{-1}(\rho)$ é uma subvariedade inicial de $M$.

(ii) Existe uma única folha simplética $\mathcal{L}$ de $(M,\{\cdot, \cdot\})$ tal que $J^{-1}(\rho) \subset \mathcal{L}$.

(iii) Seja $m \in M$ um elemento arbitrário de $J^{-1}(\rho)$. Então, $J^{-1}(\rho) \subset M_{G_{m}}$, $\operatorname{com} M_{G_{m}}:=\left\{z \in M \mid G_{z}=G_{m}\right\}$.

Seja $m \in M$ arbitrário tal que $J(m)=\rho \in M / A_{G}^{\prime}$, note que para qualquer $g \in G$, a aplicação $\Psi_{g}(\rho)=J(g \cdot m) \in M / A_{G}^{\prime}$ define uma ação de $G$ contínua em $M / A_{G}^{\prime}$ com respeito a qual $J$ é $G$-equivariante

$$
\begin{aligned}
G \times M / A_{G}^{\prime} & \longrightarrow M / A_{G}^{\prime} \\
(g, \rho) & \longmapsto \Psi_{g}(\rho)=J(g \cdot m)
\end{aligned}
$$

Proposição 7.37 Seja $G_{\rho}$ o subgrupo de isotropia do elemento $\rho \in M / A_{G}^{\prime}$ associado a ação de $G$ em $M / A_{G}^{\prime}$. Então:

(i) Existe uma única estrutura suave em $G_{\rho}$ para a qual este subgrupo se torna um subgrupo de Lie inicial de $G$.

(ii) Com esta estrutura suave para $G_{\rho}$, a ação à esquerda

$$
\begin{aligned}
\psi^{\rho}: G_{\rho} \times J^{-1}(\rho) & \longrightarrow J^{-1}(\rho) \\
(g, z) & \longmapsto \psi^{\rho}(g, z):=\psi(g, z)
\end{aligned}
$$

é suave.

No teorema a seguir, a projeção canônica no espaço de órbitas da ação de $G_{\rho}$ em $J^{-1}(\rho)$ será denotada por $\pi_{\rho}$

$$
\pi_{\rho}: J^{-1}(\rho) \longrightarrow J^{-1}(\rho) / G_{\rho}
$$

Teorema 7.38 (Redução simplética por ações de Poisson) Sejam $(M,\{\cdot, \cdot\})$ uma variedade de Poisson suave e $G$ um grupo de Lie que age em $M$, onde a ação é Poisson própria. Seja $J: M \rightarrow M / A_{G}^{\prime}$ o mapa momento ótimo associado a essa ação. Então, para qualquer $\rho \in M / A_{G}^{\prime}$ cujo subgrupo de isotropia $G_{\rho}$ age propriamente em $J^{-1}(\rho)$, o espaço de órbitas $M_{\rho}:=J^{-1}(\rho) / G_{\rho}$ é uma variedade quociente regular simplética suave com forma simplética $\omega_{\rho}$ definida por:

$$
\pi_{\rho}^{*} \omega_{\rho}(m)\left(X_{f}(m), X_{h}(m)\right)=\{f, h\}(m),
$$


para qualquer $m \in J^{-1}(\rho)$ e quaisquer $f, h \in \mathcal{C}^{\infty}(M)^{G}$. O par $\left(M_{\rho}, \omega_{\rho}\right)$ é chamado o espaço reduzido ponto ótimo em $\rho$.

Lembrando que $\pi_{\rho}^{*} \omega_{\rho}$ denota o pullback de $\omega_{\rho}$ por $\pi_{\rho}^{*}$, o pullback foi definido em (2.1).

Observação 7.39 Se $\mathcal{O}_{\rho}=G \cdot \rho \subset M / A_{G}^{\prime}$ é a $G$-órbita de $\rho \in M / A_{G}^{\prime}$, a aplicação

$$
\begin{aligned}
& J^{-1}(\rho) / G_{\rho} \longrightarrow J^{-1}\left(\mathcal{O}_{\rho}\right) / G \\
& {[m]_{\rho} \quad \longmapsto \quad[m]_{\mathcal{O}_{\rho}}}
\end{aligned}
$$

é uma bijeção, assim o quociente $M_{\mathcal{O}_{\rho}}:=J^{-1}\left(\mathcal{O}_{\rho}\right) / G$ tem uma estrutura simplética suave $\omega_{\mathcal{O}_{\rho}}$ induzida a partir de $\left(M_{\rho}, \omega_{\rho}\right)$. O par $\left(M_{\mathcal{O}_{\rho}}, \omega_{\mathcal{O}_{\rho}}\right)$ é chamado o espaço reduzido órbita ótima em $\rho$.

A folheação simplética do espaço estratificado de Poisson $M / G$ é descrita no seguinte resultado

Teorema 7.40 Seja $\psi: G \times M \rightarrow M$ uma ação de Poisson própria com mapa momento ótimo $J: M \rightarrow M / A_{G}^{\prime}$. A folha simplética da estrata $M_{(H)} / G$ passando por $[m]$ é o espaço reduzido órbita ótima $\left(J^{-1}\left(\mathcal{O}_{\rho}\right) / G, \omega_{\rho}\right)$ em $\rho=J(m)$.

Em [10] encontramos a observação abaixo, a qual será muito útil a seguir.

Observação 7.41 Assuma que a ação original $G \times M \rightarrow M$ é Hamiltoniana com mapa momento equivariante $\mu: M \rightarrow \mathfrak{g}^{*}$. Então os resultados acima nos fornecem o seguinte:

(i) $M / G$ é um espaço estratificado de Poisson por orbit types $M_{(H)} / G$;

(ii) Os espaços reduzidos $\mu^{-1}(\xi) / G_{\xi}$ são subespaços estratificados de Poisson de $M / G$ (por orbit types).

Os espaços singulares $\mu^{-1}(\xi) / G_{\xi}$ não são quocientes de variedades suaves. Quando a estrutura de Poisson é simplética, os espaços reduzidos $\mu^{-1}(\xi) / G_{\xi}$ são subespaços estratificados simpléticos, mas $M / G$ permanece um espaço estratificado de Poisson: as estratas de $\mu^{-1}(\xi) / G_{\xi}$ são as folhas simpléticas das estratas de $M / G$. 


\section{3 \\ Folheação simplética de $\left(\prod_{j=1}^{m} \mathbb{F}_{\mathbb{C}}^{j}\left(d_{1}^{j}, \ldots, d_{r_{j}}^{j}\right)\right) / S U(n)$}

Sabemos da Proposição 6.24 que a variedade bandeira $\mathbb{F}_{\mathbb{C}}\left(d_{1}, \ldots, d_{r}\right)$, onde os $d_{j}$ 's são números naturais tais que a soma é igual a um número natural $n$ fixado, pode ser vista como uma órbita coadjunta de $S U(n)$ por algum $X \in \mathfrak{s u} \mathfrak{u}^{*}(n)$, o qual possui $r$ autovalores distintos com multiplicidades descrita pela sequência $d=\left(d_{1}, \ldots, d_{r}\right)$. Sendo $\lambda_{j}$ 's os autovalores de $X$, sabemos que existe $g \in S U(n)$ tal que $g X g^{-1}=i \operatorname{diag}\left(\lambda_{1} I_{d_{1}}, \ldots, \lambda_{r} I_{d_{r}}\right)$. A órbita de $S U(n)$ por $X$ é denotamos por

$$
\mathcal{O}_{X} \simeq \mathbb{F}_{\mathbb{C}}\left(d_{1}, \ldots, d_{r}\right)
$$

Considere a variedade $\mathcal{S}$, obtida a partir do produto de $m$ órbitas coadjuntas de $S U(n)$, as quais contém os pontos $X_{j} \in \mathfrak{s u}(n), j=1, \ldots, m$

$$
\mathcal{S}=\prod_{j=1}^{m} \mathbb{F}_{\mathbb{C}}^{j}\left(d_{1}^{j}, \ldots, d_{r_{j}}^{j}\right)
$$

de modo que $d_{1}^{j}+\ldots+d_{r_{j}}^{j}=n$ para todo $j$, ou seja, $X_{j}$ tem uma quantidade $r_{j}$ de autovalores distintos com multiplicidades dada pela sequência $d^{j}=$ $\left(d_{1}^{j}, \ldots, d_{r_{j}}^{j}\right)$. Note que temos um $j$ no expoente de cada multiplicidade e um $j$ no índice de $r$, o primeiro tem a função apenas de identificação, serve para explicitar uma possível diferença entre multiplicidades de mesmo índice e expoentes distintos, por exemplo, $d_{1}^{1}$ e $d_{1}^{2}$ podem ser distintos ou não, enquanto que o segundo, o $j$ no índice de $r$, serve para enfatizar a provável diferença na quantidade de autovalores distintos de cada $X_{j}$. É importante ainda lembrar que para cada $X_{j}$ existe $g_{j} \in S U(n)$ tal que $g_{j} X_{j} g_{j}^{-1}=i \operatorname{diag}\left(\lambda_{1}^{j} I_{d_{1}^{j}}, \ldots, \lambda_{r}^{j} I_{d_{r_{j}}^{j}}\right)$.

Sejam

$$
\operatorname{pr}_{j}: \mathcal{S} \longrightarrow \mathbb{F}_{\mathbb{C}}^{j}\left(d_{1}^{j}, \ldots, d_{r_{j}}^{j}\right) \simeq \mathcal{O}_{X_{j}}, X_{j} \in \mathfrak{s u}^{*}(n), j=1 \ldots, m
$$

a projeção no $j$-ésimo fator de $\mathcal{S}$ e $\omega_{j}$ a forma simplética em $\mathcal{O}_{X_{j}}$ a qual é conhecida como a estrutura simplética de Kostant-Kirillov-Souriau. Considere a 2 -forma

$$
\omega=\sum_{j=1}^{m} \operatorname{pr}_{j}^{*} \omega_{j}
$$

do Exemplo 2.5 sabemos que $\omega$ define uma estrutura simplética em $\mathcal{S}$, dessa forma, a partir de $\omega$ podemos definir uma estrutura de Poisson em $\mathcal{S}$ dada por:

$$
\omega\left(X_{f}, X_{g}\right)=\{f, g\}
$$

onde $f$ e $g$ são funções definidas na variedade simplética $\mathcal{S}$. Sendo $\omega_{j}$ simplética, 
a estrutura de Poisson que $\omega_{j}$ define é não degenerada em $\mathcal{O}_{X_{j}}$. Portanto podemos definir em $\mathcal{S}$ uma estrutura de Poisson não degenerada, a saber, a estrutura de Poisson produto enunciada na Definição 4.10.

Considere a ação coadjunta diagonal de $S U(n)$ em $\mathcal{S}$, provamos anteriormente que esta é uma ação de Poisson, além disto, o mapa momento associado a esta ação é dado por

$$
\begin{aligned}
\mu: \mathcal{S} & \longrightarrow \mathfrak{s u}^{*}(n) \simeq \mathbb{R}^{n^{2}-1} \\
\xi & \longmapsto \mu(\xi)=\sum_{j=1}^{m} \xi_{j}
\end{aligned}
$$

onde $\xi=\left(\xi_{1}, \ldots, \xi_{m}\right)$. Note que tomando $0 \in \mathfrak{s u}^{*}(n)$, o conjunto de nível $\mu^{-1}(0) \subset \mathcal{S}$ é o seguinte

$$
\mu^{-1}(0)=\left\{\xi \in \mathcal{S} \mid \sum_{j=1}^{m} \xi_{j}=0\right\}
$$

Lembrando que

$$
\langle X, Y\rangle=\frac{1}{2} \operatorname{tr}\left(X Y^{*}\right)
$$

define um produto interno em $\mathfrak{s u}(n)$ o qual é invariante pela ação adjunta e pela equivalência das ações adjunta e coadjunta, concluímos que para qualquer $\xi_{j} \in \mathcal{O}_{X_{j}}$ temos que $\left\|\xi_{j}\right\|=\left\|X_{j}\right\|=s_{j} \in \mathbb{R}_{+}$para todo $j=1, \ldots, m$, utilizando esta informação, vemos que $\mu^{-1}(0)$ descreve o espaço dos $m$-gons de lados $v_{j}$ de comprimentos fixados $s_{j}$, onde estamos fazendo a correspondência

$$
\xi_{j} \in \mathfrak{s u}^{*}(n) \longleftrightarrow v_{j} \in \mathbb{R}^{n^{2}-1}
$$

Podemos perceber que em $\mu^{-1}(0)$ existem $m$-gons $p_{1}=\left(\xi_{1}^{1}, \ldots, \xi_{m}^{1}\right)$ e $p_{2}=$ $\left(\xi_{1}^{2}, \ldots, \xi_{m}^{2}\right)$ de tal forma que para todo $j$ tem-se $v_{j}^{i} \in \mathbb{S}_{s_{j}}^{n^{2}-2}$ para $i=1,2$, ou seja, $\left\|\xi_{j}^{1}\right\|=\left\|\xi_{j}^{2}\right\|$ para todo $j$, mas $p_{1}$ e $p_{2}$ não pertencem a mesma órbita, isto é, os lados de $p_{1}$ e $p_{2}$ são do mesmo tamanho, mas não existe $g \in S U(n)$ tal que $\mathbf{A d}_{g}^{*} p_{1}=p_{2}$, onde a ação é a coadjunta diagonal.

Foi mencionado anteriormente que $\mu^{-1}(0)$ descreve o espaço dos $m$-gons de lados $v_{j}$ de comprimento $s_{j}$, foi justificado o fato de ter lados $v_{j}$, faltou apenas a parte a respeito dos comprimentos fixados, esta se deve a tomada inicial da variedade $\mathcal{S}$, uma vez que tomada a variedade tem-se como consequência a fixação da $m$-upla $s$, o vetor de comprimento $s=\left(s_{1}, \ldots, s_{m}\right)$, o qual fornece a medida dos lados de cada $m$-gon.

É provável ainda que em $\mu^{-1}(0)$ existam $\xi$ e $\xi^{\prime}$ tais que $\xi^{\prime}=\mathbf{A d}_{g}^{*} \xi$ para algum $g \in S U(n)$, onde $\operatorname{Ad}_{g}^{*} \xi=\left(\operatorname{Ad}_{g}^{*} \xi_{1}, \ldots, \operatorname{Ad}_{g}^{*} \xi_{m}\right)$, fazemos então 
o quociente

$$
\mu^{-1}(0) / S U(n)
$$

o qual descreve

$$
M_{s}=\mu^{-1}(0) / S U(n), s=\left(s_{1}, \ldots, s_{m}\right) \in \mathbb{R}_{+}^{m}
$$

que é o espaço dos $m$-gons de lados $v_{j}$ de comprimentos fixados $s_{j}$, vale ressaltar que $M_{s}$ é uma variedade suave se, e somente se, o vetor de comprimento $s$ é genérico, ou seja, depende da tomada inicial da variedade $\mathcal{S}$, dessa forma, podemos ter $s$ genérico ou não, lembrando que $s$ é dito genérico se $\epsilon_{I}(s)=\sum_{i \in I} s_{i}-\sum_{i \in I^{c}} s_{i} \neq 0$ para todo subconjunto $I \subset\{1, \ldots, m\}$. Olhando para $p_{1}$ e $p_{2}$ como no parágrafo anterior, estes no espaço $M_{s}$ estão em classes distintas, isto é, $\left[p_{1}\right] \neq\left[p_{2}\right]$.

Mencionamos anteriormente que uma vez tomada a variedade $\mathcal{S}$ como em (7.12), estamos fixando o vetor de comprimento $s$, o qual é dado pela $m$-upla $s=\left(s_{1}, \ldots, s_{m}\right)$, isto significa que para todo $p \in \mathcal{S}$, em particular $p \in \mu^{-1}(\xi) \subset \mathcal{S}, p=\left(p_{1}, \ldots, p_{m}\right)$ satisfaz $\left\|p_{j}\right\|=s_{j}$ para todo $j$, tendo feito esta observação, analisaremos agora os quocientes $\mu^{-1}(\xi) / S U(n)_{\xi}$.

Seja $\xi \in \mathfrak{s u}^{*}(n)$, nosso intuito é estudar o quociente $\mu^{-1}(\xi) / S U(n)_{\xi}$. No caso $\xi=0, S U(n)_{0}=S U(n)$ e o quociente a ser estudado é $\mu^{-1}(0) / S U(n)$, este quociente foi estudado anteriormente, resta-nos agora o caso $\xi \neq 0$, este caso dividiremos em duas possibilidades, a primeira levando em consideração $\xi$ tendo exatamente $n$ autovalores dois a dois distintos, o que nos fornece $S U(n)_{\xi} \in(\mathbb{T}) \subset S U(n)$ e a segunda levando em consideração $\xi$ tendo exatamente $1<r<n$ autovalores dois a dois distintos, o que dará $S U(n)_{\xi}=$ $\left(G_{d}\right) \subset S U(n)$.

Assim, dependendo da escolha de $\xi \in \mathfrak{s u}^{*}(n)$ podemos facilmente determinar o estabilizador de $\xi$, caso $\xi \neq 0$ temos que $S U(n)_{\xi} \in$ $\left\{(\mathbb{T}),\left(G_{d}\right)\right\}$, onde o índice $d$ é referente a sequência que descreve as multiplicidades dos autovalores de $\xi$. Da Observação 7.41, sabemos que os espaços reduzidos $\mu^{-1}(\xi) / S U(n)_{\xi}$ são subespaços estratificados de Poisson do quociente $\mathcal{S} / S U(n)$, e desde que $\pi_{\mathcal{S}}$ é simplético, temos que $\mu^{-1}(\xi) / S U(n)_{\xi}$ são subespaços estratificados simpléticos e as suas estratas são as folhas simpléticas das estradas de $\mathcal{S} / S U(n)$.

Feito esta análise inicial, olharemos agora para o quociente $\mathcal{S} / S U(n)$, para isto precisamos estudas as órbitas da ação coadjunta diagonal de $S U(n)$ 
em $\mathcal{S}$. Esta ação é dada por

$$
\begin{aligned}
\mathbf{A d}^{*}: S U(n) \times \mathcal{S} & \longrightarrow \mathcal{S} \\
(g, \xi) & \longmapsto \operatorname{Ad}_{g}^{*}(\xi)=\left(\operatorname{Ad}_{g}^{*} \xi_{1}, \ldots, \operatorname{Ad}_{g}^{*} \xi_{m}\right)
\end{aligned}
$$

denote por $\mathcal{O}_{\xi}^{\mathcal{D}}$ a órbita coadjunta diagonal de $S U(n)$ por $\xi$, sabemos que $\mathcal{O}_{\xi}^{\mathcal{D}} \subset \mathcal{S}, \operatorname{assim} \mathcal{O}_{\xi}^{\mathcal{D}} \in \mathcal{S} / S U(n)$. É fácil ver que existe $\xi^{\prime} \in \mathcal{S}$ tal que $\psi_{g}(\xi) \neq \xi^{\prime}$ para todo $g \in S U(n)$, para isso basta tomar qualquer $\xi^{\prime} \in \mathcal{S}$ que não esteja na órbita coadjunta diagonal de $S U(n)$ por $\xi$, um $\xi^{\prime}$ que satisfaça essa condição pode ser tomado como sendo, por exemplo, $\xi^{\prime}=\left(\xi_{1}, \ldots, \operatorname{Ad}_{g}^{*} \xi_{j}, \ldots, \xi_{m}\right)$ com $g \notin S U(n)_{\xi_{j}}$, dessa forma podemos facilmente notar que $\mathcal{O}_{\xi}^{\mathcal{D}} \subsetneq \mathcal{S}$.

Se as entradas $\xi_{j}$ 's de $\xi$ não são diagonalizadas pelo mesmo $g \in S U(n)$, então em $\mathcal{O}_{\xi}^{\mathcal{D}}$ não temos o ponto em que todas as entradas são da forma diagonal, isto significa que $\mathcal{O}_{\xi}^{\mathcal{D}} \cap \mathcal{S}_{(H)}=\emptyset$, qualquer que seja o subgrupo de isotropia $H$, lembrando que as possibilidades para os subgrupos de isotropia são $\mathbb{T}$ e $G_{d}$. Uma informação a ser acrescentada é que órbitas de um grupo de Lie conexo são conexas, $\log o \mathcal{O}_{\xi}^{\mathcal{D}}$ é conexa.

O intuito é estudar a folheação simplética do espaço de órbitas $\mathcal{S} / S U(n)$, afim de entendermos essa folheação precisamos primeiramente verificar se a ação coadjunta diagonal de $S U(n)$ em $\mathcal{S}$ é livre, para obtermos essa informação iremos olhar para a variedade $\mathcal{S}$, mais especificamente para as sequências $\left(d^{j}\right)$ as quais caracterizam as entradas dos pontos de $\mathcal{S}$.

Sendo $\mathcal{S}$ como em (7.12), temos $m$ sequências $\left(d^{j}\right)$ as quais nos permitem duas possibilidades:

1. As sequências $\left(d^{j}\right)^{\prime}$ 's são todas do mesmo comprimento e diferem por uma permutação adequada, dessa forma não é difícil ver que o grupo $G_{d^{j}}$ é subgrupo de isotropia da ação, para um $j$ fixado, portanto neste caso a ação não é livre.

2. Ao menos uma das sequências não pode ser obtida por uma permutação de alguma das demais sequências, o que nos garante que a ação neste caso é livre, isto será provado a seguir.

Começamos pela segunda possibilidade. Como uma das sequências não é permutação de ao menos uma das demais, logo ou as sequências tem comprimentos distintos ou tem comprimentos iguais mas com termos diferentes, em qualquer dos casos, considere a variedade

$$
\mathcal{S}=\prod_{j=1}^{m} \mathbb{F}_{\mathbb{C}}^{j}\left(d_{1}^{j}, \ldots, d_{r_{j}}^{j}\right)
$$


satisfazendo uma dessas condições. Daí temos o seguinte.

Teorema 7.42 Considere a ação coadjunta diagonal de $S U(n)$ em $\mathcal{S}$, onde

$$
\mathcal{S}=\prod_{j=1}^{m} \mathbb{F}_{\mathbb{C}}^{j}\left(d_{1}^{j}, \ldots, d_{r_{j}}^{j}\right)
$$

tal que existem ao menos duas sequências $\left(d^{j}\right)$ e $\left(d^{k}\right)$ tal que uma não é permutação da outra. Essa ação é Poisson e admite mapa momento associado $\mu$ dado por

$$
\begin{aligned}
\mu: \mathcal{S} & \longrightarrow \mathfrak{s u}^{*}(n) \simeq \mathbb{R}^{n^{2}-1} \\
\xi & \longmapsto \mu(\xi)=\sum_{j=1}^{m} \xi_{j} .
\end{aligned}
$$

$\left(\mathcal{S}, \pi_{\mathcal{S}}, S U(n), \mu\right)$ é um $S U(n)$-espaço Hamiltoniano livre e próprio. Então $0 \in \mathfrak{s u}^{*}(n)$ é um valor regular de $\mu$ e $\mathcal{S} / / S U(n):=\mu^{-1}(0) / S U(n)$ é uma subvariedade de Poisson de $\left(\mathcal{S} / S U(n), \pi_{\mathcal{S} / S U(n)}\right)$, e uma vez que $\pi_{\mathcal{S}}$ é simplético, $\pi_{\mathcal{S} / / S U(n)}$ também é simplético e as componentes conexas de $\mathcal{S} / / S U(n)$ são folhas simpléticas de $\left(\mathcal{S} / S U(n), \pi_{\mathcal{S} / S U(n)}\right)$.

Proof. Notamos inicialmente que este resultado nada mais é do que o Teorema 4.32. O primeiro item a ser verificado neste teorema é o fato da ação coadjunta diagonal ser uma ação de Poisson, isto foi provado na Proposição 4.19, enquanto que a $S U(n)$-equivariância do mapa momento foi provada em (4.13), e uma vez que $S U(n)$ é um grupo de Lie compacto temos que a ação de $S U(n)$ em $\mathcal{S}$ é própria, portanto temos que $\left(\mathcal{S}, \pi_{\mathcal{S}}, S U(n), \mu\right)$ é um $S U(n)$-espaço Hamiltoniano próprio.

Afim de provar que a ação é livre, dentre as sequências da variedade $\mathcal{S}$ escolha $\left(d^{j}\right)$ e $\left(d^{k}\right)$ duas sequências tais que uma não pode ser obtida por uma permutação da outra. Dessa forma temos duas possibilidades.

(i) As sequências $\left(d^{j}\right)$ e $\left(d^{k}\right)$ têm comprimentos distintos.

(ii) As sequências têm o mesmo comprimento $r$ mas existe $s \in\{1, \ldots, r\}$ tal que $d_{s}^{j} \neq d_{m}^{k}$ para todo $m$.

Suponha por contradição que a ação não é livre, então existe $\xi \in \mathcal{S}$ tal que $S U(n)_{\xi} \neq\{I\}$, isto significa que existe $g \in S U(n) \backslash\{I\}$ tal que

$$
\operatorname{Ad}_{g}^{*} \xi=\left(\operatorname{Ad}_{g}^{*} \xi_{1}, \ldots, \operatorname{Ad}_{g}^{*} \xi_{m}\right)=\xi
$$

onde $\xi=\left(\xi_{1}, \ldots, \xi_{j}, \ldots, \xi_{k}, \ldots, \xi_{m}\right)$ em particular,

$$
\operatorname{Ad}_{g}^{*} \xi_{j}=\xi_{j} \text { e } \operatorname{Ad}_{g}^{*} \xi_{k}=\xi_{k}
$$


dessa forma, $g \in S U(n)_{\xi_{j}} \cap S U(n)_{\xi_{k}}$, onde $S U(n)_{\xi_{j}}$ e $S U(n)_{\xi_{k}}$ são os estabilizadores de $\xi_{j}$ e $\xi_{k}$, respectivamente, no entanto,

$$
S U(n)_{\xi_{j}} \in\left(G_{d^{j}}\right) \text { e } S U(n)_{\xi_{k}} \in\left(G_{d^{k}}\right)
$$

assim sendo, se $g \in S U(n)_{\xi_{j}}$ então $g \in S U(n)$ e é diagonal por bloco, da forma $g=\operatorname{diag}\left(a_{1}^{j}, \ldots, a_{r_{j}}^{j}\right)$ onde o bloco $a_{s}^{j} \in U\left(d_{s}^{j}\right)$ com $s=1, \ldots, r_{j}$. Como consequência da condição inicial das sequências $d^{j}=\left(d_{1}^{j}, \ldots, d_{r_{j}}^{j}\right)$ e $d^{k}=\left(d_{1}^{k}, \ldots, d_{r_{k}}^{k}\right)$ que tomamos, onde não existe uma permutação $p$ tal que $p\left(d^{j}\right)=d^{k}$, segue que a sequência das dimensões dos blocos dos elementos de $S U(n)_{\xi_{j}}$ não existe em nenhum elemento de $S U(n)_{\xi_{k}}$, isto significa que $g \notin S U(n)_{\xi_{k}}$ e portanto $S U(n)_{\xi_{j}} \cap S U(n)_{\xi_{k}}$ é vazia, gerando assim uma contradição, portanto a ação coadjunta diagonal de $S U(n)$ em $\mathcal{S}$ é uma ação livre. Portanto $\left(\mathcal{S}, \pi_{\mathcal{S}}, S U(n), \mu\right)$ é um $S U(n)$-espaço Hamiltoniano livre e próprio. Feito isto, a última parte segue do Teorema 4.32.

Estudaremos agora a primeira possibilidade, na qual a ação é própria mas não é livre, devido a isto usaremos estratificação para compreendermos a folheação simplética de $\mathcal{S} / S U(n)$.

Para este caso temos

$$
\mathcal{S}=\prod_{j=1}^{m} \mathbb{F}_{\mathbb{C}}^{j}\left(d_{1}^{j}, \ldots, d_{r}^{j}\right),
$$

note que neste caso $r$ não possui o índice $j$, uma vez que as sequências $d^{j}$ 's dos argumentos das variedades bandeira $\mathbb{F}_{\mathbb{C}}^{j}\left(d_{1}^{j}, \ldots, d_{r}^{j}\right)$ possuem o mesmo comprimento, além disso, essas sequências diferem por uma permutação, dessa forma, dado $\xi \in \mathcal{S}$, o estabilizador de qualquer entrada $\xi_{j}$ de $\xi$ pertence a classe de conjugação do subgrupo $G_{d^{j}}$ em que $G_{d^{j}}=S U(n) \cap E_{d^{j}}$ onde $d^{j}=\left(d_{1}^{j}, \ldots, d_{r}^{j}\right)$, o estabilizador coincidirá com $G_{d^{j}}$ se $\xi_{j}$ estiver na forma $\xi_{j}=i \operatorname{diag}\left(\lambda_{1}^{j} I_{d_{1}^{j}}, \ldots, \lambda_{r}^{j} I_{d_{r}^{j}}\right)$.

Pelo exposto, vemos que a ação coadjunta diagonal de $S U(n)$ em $\mathcal{S}$ não é livre, e além disso, os estabilizadores desta ação possuem uma característica bem determinada, todos estão na classe de conjugação de $G_{d^{j}}$ para um $j$ fixado, esta característica é devida ao mesmo comprimento das sequências $d^{j}$ 's e dessas sequências diferirem por uma permutação, como mencionado anteriormente.

Dados a sequência $d=\left(d_{1}, \ldots, d_{r}\right)$, a qual difere das sequências $d^{j}$ 's por uma permutação, e o subgrupo de isotropia $G_{d}=S U(n) \cap E_{d}$, a subvariedade com tipo de isotropia $G_{d}$, denotada por $\mathcal{S}_{G_{d}}$ é expressa por

$$
\mathcal{S}_{G_{d}}=\left\{\xi \in \mathcal{S} \mid S U(n)_{\xi}=G_{d}\right\}
$$


isto significa que o subgrupo $G_{d}$ fixa todas as entradas $\xi_{j}$ de $\xi$, implicando que $\xi_{j}=i \operatorname{diag}\left(\lambda_{1}^{j} I_{d_{1}}, \ldots, \lambda_{r}^{j} I_{d_{r}}\right)$, onde $\lambda_{r}^{j} \neq \lambda_{s}^{j}$ se $r \neq s$ e com a condição de que $\lambda_{1}^{j} d_{1}+\ldots+\lambda_{r}^{j} d_{r}=0$ para todo $j=1, \ldots, m$, esta última se deve a $\xi_{j} \in \mathfrak{s u}{ }^{*}(n)$ para todo $j$.

A subvariedade com tipo de órbita $\left(G_{d}\right), \mathcal{S}_{\left(G_{d}\right)}$, é dada por

$$
\mathcal{S}_{\left(G_{d}\right)}=\left\{\xi \in \mathcal{S} \mid S U(n)_{\xi} \in\left(G_{d}\right)\right\}
$$

isto significa que dado $\xi \in \mathcal{S}_{\left(G_{d}\right)}$, existe $g \in S U(n)$ tal que $S U(n)_{\xi}=g G_{d} g^{-1}$, $\operatorname{logo} g G_{d} g^{-1}$ estabiliza todas as entradas $\xi_{j}$ de $\xi$, como consequência da Proposição 7.6 temos que

$$
\mathbf{A d}_{g^{-1}}^{*} \xi:=\left(\operatorname{Ad}_{g^{-1}}^{*} \xi_{1}, \ldots, \operatorname{Ad}_{g^{-1}}^{*} \xi_{m}\right)=\xi_{0}
$$

onde $\xi_{0} \in \mathcal{S}_{G_{d}}$. Note que todos os pontos da órbita coadjunta diagonal de $S U(n)$ por $\xi$ pertencem a subvariedade $\mathcal{S}_{\left(G_{d}\right)}$, isto é,

$$
\mathcal{O}_{\xi}^{\mathcal{D}} \simeq \mathcal{D}_{\xi}\left(\prod_{j=1}^{m} \mathbb{F}_{\mathbb{C}}^{j}\left(d_{1}, \ldots, d_{r}\right)\right) \subset \mathcal{S}_{\left(G_{d}\right)}
$$

e ainda, toda órbita $\mathcal{O}_{\xi}^{\mathcal{D}} \operatorname{com} \xi \in \mathcal{S}_{\left(G_{d}\right)}$ contém um ponto $\xi_{0} \in \mathcal{S}_{G_{d}}$ como exibido acima. Notamos que o espaço de órbitas $\mathcal{S}_{\left(G_{d}\right)} / S U(n)$ é formado pelas órbitas $\mathcal{O}_{\xi}^{\mathcal{D}}, \xi \in \mathcal{S}_{\left(G_{d}\right)}$ as quais são conexas pois $S U(n)$ o é.

Observação 7.43 Existe uma diferença sutil entre os espaços de órbita $\mathcal{S} / S U(n)$ e $\mathcal{S}_{\left(G_{d}\right)} / S U(n)$, no primeiro podemos pensar nas órbitas diagonais a partir de um ponto qualquer $\xi$ de $\mathcal{S}$ o qual não está condicionado a uma diagonalização de suas entradas pelo mesmo $g \in S U(n)$, isto nos diz que a órbita coadjunta diagonal de $S U(n)$ por $\xi$ não necessariamente contém o ponto que é a diagonalização de todas as entradas de $\xi$, diferentemente do segundo caso, no qual todo ponto de $\mathcal{S}_{\left(G_{d}\right)}$ tem a diagonalização de suas entradas efetuada pelo mesmo $g \in S U(n)$. E ainda, uma vez que $\mathcal{S}_{\left(G_{d}\right)} \subset \mathcal{S}$ segue que $\mathcal{S}_{\left(G_{d}\right)} / S U(n) \subset \mathcal{S} / S U(n)$

No teorema a seguir a variedade $\mathcal{S}$ é dada por

$$
\mathcal{S}=\prod_{j=1}^{m} \mathbb{F}_{\mathbb{C}}^{j}\left(d_{1}^{j}, \ldots, d_{r}^{j}\right)
$$

onde $d_{1}^{j}+\ldots+d_{r_{j}}^{j}=n$ para todo $j, \mathcal{S}$ admite uma estrutura de Poisson produto $\pi_{\mathcal{S}}$ a qual é não degenerada. Nesse resultado chegaremos a conclusão que $\mathcal{S} / S U(n)$ é um espaço estratificado de Poisson onde as estratas são as 
componentes conexas de $\mathcal{S}_{(H)} / S U(n)$ de tal forma que $H$ é subgrupo de isotropia da ação coadjunta diagonal de $S U(n)$ em $\mathcal{S}$.

Teorema 7.44 Considere a ação coadjunta diagonal de $S U(n)$ em $\mathcal{S}$, onde

$$
\mathcal{S}=\prod_{j=1}^{m} \mathbb{F}_{\mathbb{C}}^{j}\left(d_{1}^{j}, \ldots, d_{r}^{j}\right)
$$

esta é uma ação Poisson e admite um mapa momento associado $\mu$ dado por

$$
\begin{aligned}
\mu: \mathcal{S} & \longrightarrow \mathfrak{s u}^{*}(n) \simeq \mathbb{R}^{n^{2}-1} \\
\xi & \longmapsto \mu(\xi)=\sum_{j=1}^{m} \xi_{j} .
\end{aligned}
$$

Nessas condições $\left(\mathcal{S}, \pi_{\mathcal{S}}, S U(n), \mu\right)$ é um $S U(n)$-espaço Hamiltoniano próprio, pois $\mu$ é $S U(n)$-equivariante. Além disso $\mathcal{S} / S U(n)$ é um espaço estratificado de Poisson em que as estratas são dadas pelas componentes conexas de $\mathcal{S}_{(H)} / S U(n)$, onde $H$ são subgrupos de isotropia da ação, e os espaços reduzidos $\mu^{-1}(\xi) / S U(n)_{\xi}$ são subespaços estratificados simpléticos cujas estratas são as folhas simpléticas das estratas de $\mathcal{S} / S U(n)$.

Proof. A prova de que $\left(\mathcal{S}, \pi_{\mathcal{S}}, S U(n), \mu\right)$ é um $S U(n)$-espaço Hamiltoniano próprio foi feita no Teorema 7.42. Pelos comentários que antecedem este resultado, sabemos que a ação coadjunta diagonal de $S U(n)$ em $\mathcal{S}$ não é livre. Note que o Teorema 4.32 não se aplica pois neste caso a ação coadjunta diagonal de $S U(n)$ em $\mathcal{S}$ não é livre mas ainda temos $\left(\mathcal{S}, \pi_{\mathcal{S}}, S U(n), \mu\right)$ um $S U(n)$-espaço Hamiltoniano próprio. A ação não sendo livre significa que o subgrupo de isotropia não é o trivial, note que observando a variedade $\mathcal{S}$, a qual está fixada, podemos facilmente determinar um subgrupo de isotropia a partir da sequência $(d)$ das multiplicidades, assim o subgrupo de isotropia é dado por

$$
H=\left\{g \in S U(n) \mid g=\operatorname{diag}\left(a_{1}, \ldots, a_{r}\right), a_{j} \in U\left(d_{j}\right)\right\}, d_{1}+\cdots+d_{r}=n
$$

todos os demais subgrupos de isotropia estão na classe de conjugação de $H$.

Sabendo quais são os subgrupos de isotropia, podemos determinar as variedades com tipo de isotropia $H$ e a com tipo de órbita $(H)$, as quais são dadas por:

$$
\mathcal{S}_{H}=\left\{X \in \mathcal{S} \mid X=\left(X_{1}, \ldots, X_{m}\right), X_{j}=i \operatorname{diag}\left(\lambda_{1}^{j} I_{d_{1}}, \ldots, \lambda_{r}^{j} I_{d_{r}}\right)\right\}
$$

enquanto que a subvariedade com tipo de órbita $(H), \mathcal{S}_{(H)}$ é formada pelos pontos $\xi \in \mathcal{S}$ tal que para todo $j$ temos $S U(n)_{\xi_{j}}=g H g^{-1}$ para algum 
$g \in S U(n)$, ou seja,

$$
g^{-1} \xi_{j} g=i \operatorname{diag}\left(\lambda_{1}^{j} I_{d_{1}}, \ldots, \lambda_{r}^{j} I_{d_{r}}\right)
$$

note que se $\xi \in \mathcal{S}_{(H)}$, então a órbita coadjunta diagonal de $S U(n)$ por $\xi$ está contida em $\mathcal{S}_{(H)}$ e é dada por

$$
\mathcal{O}_{\xi}^{\mathcal{D}}=\mathcal{D}_{\xi}\left(\prod_{j=1}^{m} \mathbb{F}_{\mathbb{C}}^{j}\left(d_{1}, \ldots, d_{r}\right)\right)
$$

Pelo Teorema 7.12 temos que $\mathcal{S}$ é um espaço estratificado, cujas estratas são as componentes conexas de $\mathcal{S}_{(H)}$ e as componentes conexa de $\mathcal{S}_{(H)} / S U(n)$ formam uma estratificação do espaço $\mathcal{S} / S U(n)$, onde $H$ é um subgrupo de isotropia da ação, e o Teorema 7.22 nos garante que esta última estratificação é uma estratificação de Poisson de $\left(\mathcal{S} / S U(n),\{\cdot, \cdot\}_{\mathcal{S} / S U(n)}\right)$. Uma vez que a estrutura de Poisson $\pi_{\mathcal{S}}$ em $\mathcal{S}$ é simplética pois ela vem da estrutura simplética definida em $\mathcal{S}$, podemos aplicar a Observação 7.41, a qual nos garante que $\mu^{-1}(\xi) / S U(n)_{\xi}$ são subespaços estratificados simpléticos e as estratas de $\mu^{-1}(\xi) / S U(n)_{\xi}$ são as folhas simpléticas das estratas de $\mathcal{S} / S U(n)$.

Temos que $\mu^{-1}(\xi) \subset \mathcal{S}$ e desde que $\xi \in \mathfrak{s u}(n)$, segue que $S U(n)_{\xi} \in\left(G_{d}\right)$, onde $d$ é a sequência que descreve as multiplicidades dos autovalores de $\xi$, vale ressaltar que a sequência $(d)$ das multiplicidades dos autovalores de $\xi$ pode ser diferente da sequência que consta no teorema acima, isso depende da escolha do nível $\xi \in \mathfrak{s u}^{*}(n)$.

Sabemos que $\mu^{-1}(\xi)=\left\{\xi^{\prime} \in \mathcal{S} \mid \xi_{1}^{\prime}+\cdots+\xi_{m}^{\prime}=\xi\right\}$, dessa forma, podemos ver $\mu^{-1}(\xi)$ como o espaço dos $(m+1)$-gons em $\mathcal{S}$ de lados de comprimentos fixados $\left(s_{1}, \ldots, s_{m}, s_{\xi}\right)$, de tal forma que agora temos uma sequência de comprimentos com $m+1$ entradas com a última entrada determinada a partir da escolha do nível $\xi$, ou seja, $s_{\xi}=\|\xi\|$.

Para o nível zero, $\mu^{-1}(0) \subset \mathcal{S}$, temos que $S U(n)_{0}=S U(n)$, caso a variedade $\mathcal{S}$ satisfaça a condição do vetor de comprimento $s=\left(s_{1}, \ldots, s_{m}\right)$ ser genérico, ou seja,

$$
\epsilon_{I}(s)=\sum_{i \in I} s_{i}-\sum_{i \in I^{c}} s_{i} \neq 0, I \subset\{1, \ldots, m\}
$$

teremos que a variedade quociente $\mu^{-1}(0) / S U(n)$ é suave e descreve o espaço de polígonos de $m$ lados de comprimento fixado. 


\subsection{1}

\section{Espaço de moduli de polígonos para $S U(2)$ e $S U(n)$}

No Exemplo 7.27 estudamos a ação coadjunta diagonal de $S U(2)$ na variedade obtida pelo produto de $m$ órbitas coadjuntas, $\mathcal{S}=\prod_{j=1}^{m} \mathbb{S}_{\left|\lambda_{j}\right|}^{2}$, nesse estudo analisamos as órbitas da ação coadjunta diagonal, vimos que a ação não é livre mas é própria devido $S U(2)$ ser um grupo compacto, pelo o que foi estudado chegamos a conclusão de que todos os subgrupos de isotropia da ação estão na classe de conjugação de $\mathbb{T}$, onde

$$
\mathbb{T}=\{g \in S U(2) \mid g=\operatorname{diag}(\alpha, \bar{\alpha}), \alpha \in U(1)\},
$$

lembrando ainda que o mapa momento associado a esta ação é denotado por $\mu$ e sua expressão está fornecida em (7.6). Pelo o que foi mostrado na Seção 7.3 , podemos facilmente concluir que $\left(\mathcal{S}, \pi_{\mathcal{S}}, S U(2), \mu\right)$ é um $S U(2)$-espaço Hamiltoniano próprio, desta forma, o Teorema 7.44 garante que $\mathcal{S} / S U(2)$ é um espaço estratificado de Poisson onde as estratas são dadas pelas componentes conexas $c\left(\mathcal{S}_{(\mathbb{T})} / S U(2)\right)$, além disto, os espaços reduzidos $\mu^{-1}(\xi) / S U(2)_{\xi}$ são subespaços estratificados simpléticos cujas estratas são as folhas simpléticas das estratas de $\mathcal{S} / S U(2)$. Em particular, $\mu^{-1}(0) / S U(2)$, que é o espaço de moduli de polígonos de $m$-lados.

Agora para o caso $S U(n)$, este foi abordado na Seção 7.3, onde foi estudado a ação de $S U(n)$ em $\mathcal{S}=\prod_{j=1}^{m} \mathbb{F}_{\mathbb{C}}^{j}\left(d_{1}^{j}, \ldots, d_{r_{j}}^{j}\right)$, o estudo foi feito em duas situações possíveis, uma em que a ação é livre e própria e outra em que a ação é apenas própria.

Para a primeira situação, em que a ação é livre e própria, o Teorema 7.42 nos fornece que $\mu^{-1}(0) / S U(n)$ é uma subvariedade de Poisson de $\left(\mathcal{S} / S U(n), \pi_{\mathcal{S} / S U(n)}\right)$ e como $\mathcal{S}$ é uma variedade simplética, segue $\mu^{-1}(0) / S U(n)$ também é simplética e as componentes conexas $c\left(\mu^{-1}(0) / S U(n)\right)$ são folhas simpléticas de $\left(\mathcal{S} / S U(n), \pi_{\mathcal{S} / S U(n)}\right)$, lembrando que $\mu^{-1}(0) / S U(n)$ é o espaço de moduli de polígonos de $m$ lados fixados.

Para a segunda situação em que a ação é apenas própria, o Teorema 7.44 nos fornece a informação de que os espaços reduzidos $\mu^{-1}(\xi) / S U(n)_{\xi}$ são subespaços estratificados simpléticos cujas estratas são folhas simpléticas das estratas de $\mathcal{S} / S U(n)$. 


\section{4}

Subvariedades especiais de $M=\prod_{j=1}^{m} \mathfrak{s u}^{*}(n)$

Na seção 6.4 iniciamos o estudo da ação de $S U(n)$ em $M$, estudamos as órbitas da ação coadjunta diagonal de $S U(n)$. Agora faremos uso de estratificação para estudarmos o quociente $M / S U(n)$ e posteriormente iremos decompor $M$ em uma união de dois tipos de subvariedades especiais as quais estão relacionadas com a ação de $S U(n)$ ser ou não livre.

A variedade $M$ está equipada com a estrutura de Poisson produto, pelo Teorema 7.12 sabemos que podemos escrever $M$ como uma união disjunta de subvariedades com tipo de órbita $\left(G_{d}\right)$ onde $G_{d}$ é um subgrupo de isotropia da ação coadjunta diagonal de $S U(n)$ em $M$, ou seja, $G_{d}$ é dado por

$$
G_{d}=\left\{g \in S U(n) \mid g=\operatorname{diag}\left(a_{1}, \ldots, a_{r}\right), a_{j} \in U\left(d_{j}\right)\right\},
$$

$d=\left(d_{1}, \ldots, d_{r}\right), d_{1}+\cdots+d_{r}=n$. Aqui estamos deixando de mencionar o subgrupo $H$, pois consideramos que $G_{d}$ engloba o caso em que o subgrupo é $H$, bastando para isto tomar a sequência $d=(1, \ldots, 1)$. Pelo o que abordamos anteriormente, sabemos que $G_{d}$ estabiliza pontos de $M$ os quais possuem todas as entradas da forma

$$
\xi=\left(\xi_{1}, \ldots, \xi_{m}\right), \xi_{j}=i \operatorname{diag}\left(\lambda_{1}^{j} I_{d_{1}}, \ldots, \lambda_{r}^{j} I_{d_{r}}\right), \forall j
$$

ou seja, $G_{d}$ estabiliza pontos em que todas as entradas são diagonais por blocos e tenham exatamente $r$ autovalores distintos e que os autovalores tenham multiplicidades iguais a $d_{1}, \ldots, d_{r}$, respectivamente.

Note que a partir da sequência $(d)$ podemos obter alguns pontos de $M$ que estão relacionados com esta sequência, esses pontos são aqueles que tem as multiplicidades dos autovalores de suas entradas descrita por essa sequência, dessa forma, todos os pontos de $M$ que estão na mesma órbita coadjunta diagonal de $S U(n)$ que passam por pontos da forma dada em (7.14) satisfazem essa relação com a sequência $(d)$, ou seja, eles possuem as mesmas multiplicidades de autovalores. Sabemos que a subvariedade com tipo de órbita $\left(G_{d}\right)$ é formada exatamente por todos os pontos que estão nas órbitas coadjuntas diagonais de $S U(n)$ que passam pelos pontos de $M_{G_{d}}$, ou seja, se $\xi^{\prime} \in M_{\left(G_{d}\right)}$, então existe $g \in S U(n)$ tal que $g \xi^{\prime} g^{-1}=\xi$ com $\xi \in M_{G_{d}}$, dessa forma se denotarmos $\mathcal{O}_{\xi}^{\mathcal{D}}$ a órbita coadjunta diagonal de $S U(n)$ por $\xi$ teremos que $\mathcal{O}_{\xi}^{\mathcal{D}} \subset M_{\left(G_{d}\right)}$.

Quanto aos subgrupos de isotropia, os subgrupos $G_{d}$ são caracterizados pelas sequências das multiplicidades dos autovalores, essas sequências podem 
ser de diversos tipos, inclusive pode ser da forma $(1, \ldots, 1)$ o que nos fornece que nesse caso $G_{d}$ é o toro em $S U(n)$. Note ainda que os pontos de $M$ nos quais a ação é livre podem ser recuperados pelo subgrupo trivial, este subgrupo é aquele que contém apenas o elemento identidade. Portanto podemos escrever

$$
M=\prod_{j=1}^{m} \mathfrak{s u}^{*}(n)=\bigcup_{\left(G_{d}\right)} M_{\left(G_{d}\right)}
$$

vale ressaltar que esta é uma união disjunta.

O nosso intuito é estudar o quociente $M / S U(n)$, este quociente de acordo com a Proposição 8.3 é Hausdorff, e possui uma estrutura de variedade estratificada, lembrando que sendo a ação não livre, temos as seguintes estratificações

$$
M=\bigcup_{\left(G_{d}\right)} c\left(M_{\left(G_{d}\right)}\right) \text { e } M / S U(n)=\bigcup_{\left(G_{d}\right)} c\left(M_{\left(G_{d}\right)} / S U(n)\right)
$$

onde $c(X)$ denota a componente conexa de $X$. Note que dependendo do subgrupo de isotropia $G_{d}$, podemos descrever os pontos de $M_{\left(G_{d}\right)}$, ou seja, sendo $G_{d}$ o subgrupo teremos que cada ponto de $M_{\left(G_{d}\right)}$ pertence a alguma órbita do tipo

$$
\mathcal{O}_{\xi}^{\mathcal{D}}=\mathcal{D}_{\xi}\left(\prod_{j=1}^{m} \mathbb{F}_{\mathbb{C}}^{j}\left(d_{1}, \ldots, d_{r}\right)\right) .
$$

para algum $\xi$ da forma dada em (7.14).

Para $G_{d}=\{I\}$ temos que se $\xi \in M_{G_{d}}$, então existe $X \in M$ de tal forma que $\xi \in \mathcal{O}_{X}^{\mathcal{D}}$, em particular, todos os pontos de $\mathcal{O}_{X}^{\mathcal{D}}$ pertencem a $M_{G_{d}}$ uma vez que $\mathcal{O}_{X}^{\mathcal{D}} \subset M_{\left(G_{d}\right)}=M_{G_{d}}$ para $G_{d}=\{I\}$, esse $X$ não é qualquer, $X$ possui a seguinte característica: É uma $m$-upla $X=\left(X_{1}, \ldots, X_{m}\right)$ onde $X_{j}=i \operatorname{diag}\left(\lambda_{1}^{j} I_{d_{1}^{j}}, \ldots, \lambda_{r_{j}}^{j} I_{d_{r_{j}}^{j}}\right)$ de tal forma que dentre as sequências $\left(d^{j}\right)$ que descrevem a multiplicidade dos autovalores de cada entrada, exista uma que não é a permutação de pelo menos uma das demais sequências, ou seja, a ação não é livre em $X$. Note que se $\xi \in \mathcal{O}_{X}^{\mathcal{D}}$ então os autovalores de $\xi_{j}$ e $X_{j}$ são iguais e possuem as mesmas multiplicidades, logo existe $g \in S U(n)$ tal que $g \cdot X_{j}=\xi_{j}$, para todo $j$, onde a ação é a coadjunta diagonal, ou seja,

$$
\mathcal{O}_{\xi}^{\mathcal{D}}=\mathcal{O}_{X}^{\mathcal{D}}=\mathcal{D}_{X}\left(\prod_{j=1}^{m} \mathbb{F}_{\mathbb{C}}^{j}\left(d_{1}^{j}, \ldots, d_{r_{j}}^{j}\right)\right)
$$

A partir desta análise concluímos que o estudo da estratificação da variedade $\mathcal{S}=\prod_{j=1}^{m} \mathbb{F}_{\mathbb{C}}^{j}\left(d_{1}^{j}, \ldots, d_{r_{j}}^{j}\right)$ é suficiente para o estudo da estratificação de $M=$ 
$\prod_{j=1}^{m} \mathfrak{s u}^{*}(n)$, assim sendo, considere a variedade $M$, dado $X \in M$ temos $X=\left(X_{1}, \ldots, X_{m}\right)$ com $X_{j} \in \mathfrak{s u}^{*}(n)$, sabemos que $X_{j}$ tem no máximo $n$ autovalores distintos, suponha sem perda de generalidade que $X_{j}$ tenha exatamente $r_{j} \leq n$ autovalores distintos com multiplicidades dada pela sequência $d^{j}=\left(d_{1}^{j}, \ldots, d_{r_{j}}^{j}\right)$, isto significa que $X_{j} \in \mathbb{F}_{\mathbb{C}}\left(d_{1}^{j}, \ldots, d_{r_{j}}^{j}\right)$ o que nos garante que $X \in \mathcal{S}=\prod_{j=1}^{m} \mathbb{F}_{\mathbb{C}}^{j}\left(d_{1}^{j}, \ldots, d_{r_{j}}^{j}\right)$, dessa forma, para qualquer $X \in M$ existe uma subvariedade $\mathcal{S}$ de $M$, tal que $X \in \mathcal{S}$ e portanto podemos escrever $M$ como uma união disjunta das subvariedades $\mathcal{S}$, pois se temos duas subvariedades $\mathcal{S}_{1}$ e $\mathcal{S}_{2}$ distintas, então elas não tem pontos em comum. Iremos classificar as subvariedades $\mathcal{S}$ de acordo com a sequência $\left(d^{j}\right)$, da seguinte forma:

- Dizemos que $\mathcal{S}$ é do tipo $F$ se entre as sequências $\left(d^{j}\right)$ de todas as entradas existir ao menos uma que não é a permutação de ao menos uma das demais sequências. As subvariedades $\mathcal{S}$ do tipo $F$ englobam todas as subvariedades que possuam sequências de mesmo comprimento mas com pelo menos uma dessas sequências não sendo permutação de ao menos uma das demais sequências e aquelas subvariedades que possuam sequências de comprimentos distintos, em qualquer dos casos temos

$$
\mathcal{S}=\prod_{j=1}^{m} \mathbb{F}_{\mathbb{C}}^{j}\left(d_{1}^{j}, \ldots, d_{r_{j}}^{j}\right)
$$

- Dizemos que $\mathcal{S}$ é do tipo $P$ se as sequências $\left(d^{j}\right)$ de todas as entradas diferem por uma permutação, neste caso temos

$$
\mathcal{S}=\prod_{j=1}^{m} \mathbb{F}_{\mathbb{C}}^{j}\left(d_{1}^{j}, \ldots, d_{r}^{j}\right)
$$

ou seja, todas as sequências possuem o mesmo comprimento.

Note que por essa classificação existem apenas dois tipos de subvariedades $\mathcal{S}$, as de tipo $F$ e de tipo $P$, a classificação foi feita com base na ação coadjunta diagonal de $S U(n)$ em pontos de $\mathcal{S}$ do tipo $F$ ser própria e livre enquanto que nos pontos de $\mathcal{S}$ do tipo $P$ garantimos apenas que a ação é própria.

O grupo de Lie $S U(n)$ é conexo, logo as subvariedades $\mathcal{S}$ são todas conexas. Temos ainda que $M$ pode ser escrita como

$$
M=\bigcup_{\left(G_{d}\right)} c\left(M_{\left(G_{d}\right)}\right)
$$


onde $G_{d}$ é subgrupo de isotropia para a ação. As subvariedades $\mathcal{S}$ do tipo $P$ e do tipo $F$ sempre intersectam alguma componente conexa $c\left(M_{\left(G_{d}\right)}\right)$ e $c\left(M_{(I)}\right)$, respectivamente, existe a interseção mas não necessariamente a inclusão, isto é o que será abordado nos próximos dois parágrafos.

Seja $\mathcal{S}$ uma subvariedade do tipo $P$, a qual denotaremos por $\mathcal{S}_{P}$, então existe uma sequência $(d)$ que descreve, a menos de uma permutação adequada para cada ponto, as multiplicidades de todas as entradas dos pontos dessa subvariedade e essa sequência também nos fornece um subgrupo de $S U(n)$ que é subgrupo de isotropia para a ação, mais precisamente o subgrupo $G_{d}$, assim, existe uma componente conexa de $M_{\left(G_{d}\right)}$ que contém pontos de $\mathcal{S}_{P}$, seja $\xi$ um desses pontos, temos que $\mathcal{O}_{\xi}^{\mathcal{D}} \subset c\left(M_{\left(G_{d}\right)}\right) \cap \mathcal{S}_{P}$, onde

$$
\mathcal{O}_{\xi}^{\mathcal{D}}=\mathcal{D}_{\xi}\left(\prod_{j=1}^{m} \mathbb{F}_{\mathbb{C}}^{j}\left(d_{1}, \ldots, d_{r}\right)\right) \subset \prod_{j=1}^{m} \mathbb{F}_{\mathbb{C}}^{j}\left(d_{1}^{j}, \ldots, d_{r}^{j}\right)=\mathcal{S}_{P}
$$

aqui garantimos a interseção de alguns pontos, no entanto, se $\xi=\left(\xi_{1}, \ldots, \xi_{m}\right)$ é o ponto mencionado acima, temos que $\xi^{\prime}=\left(\xi_{1}, \ldots, \operatorname{Ad}_{g}^{*} \xi_{k}, \ldots, \xi_{m}\right) \in \mathcal{S}_{P}$ mas este ponto não pertence a componente conexa mencionada acima desde que $g \notin S U(n)_{\xi_{k}}$, isto nos garante que a interseção ocorre mas a igualdade não.

Vamos agora esclarecer a interseção das variedades $\mathcal{S}$ do tipo $F$, a qual denotaremos por $\mathcal{S}_{F}$, com as componentes conexas $c\left(M_{(I)}\right)$. Para qualquer $\xi \in \mathcal{S}_{F}$ temos que $S U(n)_{\xi}=\{I\}$, isto significa que $\xi \in M_{(I)}$, seja $c\left(M_{(I)}\right)$ a componente conexa de $M_{(I)}$ que contém $\xi$, exibimos assim a interseção mencionada. A variedade $\mathcal{S}_{F}$ é conexa, podemos assim facilmente concluir que $\mathcal{S}_{F} \subset M_{(I)}$.

Portanto, podemos escrever $M$ da seguinte forma

$$
M=\left(\cup \mathcal{S}_{F}\right) \bigcup\left(\cup \mathcal{S}_{P}\right)
$$

Assim, para estudar a ação de $S U(n)$ em $M$, podemos restringir a ação para cada subvariedade $\mathcal{S}$ do tipo $F$ ou do tipo $P$, e esses casos foram estudados exatamente nos Teoremas 7.42 e 7.44 , respectivamente.

\section{5}

\section{Retornando aos exemplos}




\subsection{1}

Folha simplética da estrata $c\left(\mathfrak{s u}^{*}(2)_{(\mathbb{T})} / S U(2)\right)$

Nesse exemplo temos $S U(2)$ agindo em $\mathfrak{s u}^{*}(2)$ pela ação coadjunta, neste caso a distribuição generalizada $A_{S U(2)}^{\prime}$ é dada por

$$
A_{S U(2)}^{\prime}=\left\{\operatorname{ad}_{X}^{*} \xi \mid X \in \mathfrak{s u}(2), \xi \in \mathfrak{s u}^{*}(2)\right\}
$$

sabemos que as folhas do dual de uma álgebra de Lie coincidem com as órbitas coadjuntas, resultado este que foi provado no Teorema 4.17, portanto, se $\rho \in \mathfrak{s u}^{*}(2) / A_{S U(2)}^{\prime}$ então existe $\xi \in \mathfrak{s u}^{*}(2)$ tal que $\rho=\mathcal{O}_{\xi}$, onde $\mathcal{O}_{\xi}$ é a órbita coadjunta de $S U(2)$ por $\xi$, mais precisamente,

$$
\rho \simeq \mathbb{F}_{\mathbb{C}}(1,1) \simeq \mathbb{C P}^{1} \simeq \mathbb{S}_{|\lambda|}^{2},
$$

onde $\lambda$ é o autovalor de $\xi$. O mapa momento ótimo é uma aplicação definida em

$$
J: \mathfrak{s u}^{*}(2) \longrightarrow \mathfrak{s u}^{*}(2) / A_{S U(2)}^{\prime}
$$

note que para todo $\rho \in \mathfrak{s u}^{*}(2) / A_{S U(2)}^{\prime}$ temos que $S U(2)_{\rho}=S U(2)$ pois a ação é a coadjunta e a órbita coadjunta coincide com a folha simplética, assim, $S U(2) \cdot \rho=\mathcal{O}_{\rho}=\rho \in \mathfrak{s u}^{*}(2) / A_{S U(2)}^{\prime}$, dessa forma as condições da Observação 7.39 estão sendo satisfeitas e portanto o quociente

$$
M_{\rho}=M_{\mathcal{O}_{\rho}}:=J^{-1}\left(\mathcal{O}_{\rho}\right) / S U(2)=J^{-1}(\rho) / S U(2)
$$

tem uma estrutura simplética. O Teorema 7.40 mostra que o espaço reduzido $\left(J^{-1}(\rho), \omega_{\rho}\right)$ é folha simplética da estrata $\mathbb{S}_{\left|\lambda_{m}\right|}^{2}=c\left(\mathfrak{s u}^{*}(2)_{(H)} / S U(2)\right)$ passando por $[m]$ em que $\rho=J(m)$ e $\lambda_{m}$ é autovalor de $m$.

\subsection{2}

Folha simplética da estrata $c\left(\mathfrak{s u}^{*}(n)_{\left(G_{d^{j}}\right)} / S U(n)\right)$

Nesses exemplos temos $S U(n)$ agindo em $\mathfrak{s u}^{*}(n)$ pela ação coadjunta, nesses casos

$$
A_{S U(n)}^{\prime}=\left\{\operatorname{ad}_{X}^{*} \xi \mid X \in \mathfrak{s u}(n), \xi \in \mathfrak{s u}^{*}(n)\right\}
$$

sabemos que as folhas do dual de uma álgebra de Lie coincidem com as órbitas coadjuntas, portanto, se $\rho \in \mathfrak{s u}^{*}(n) / A_{S U(n)}^{\prime}$ então existe $\xi \in \mathfrak{s u}^{*}(n)$ tal que $\rho=\mathcal{O}_{\xi}$, onde $\mathcal{O}_{\xi}$ é a órbita coadjunta de $S U(n)$ que passa por $\xi$, mais precisamente, $\rho \simeq \mathbb{F}_{\mathbb{C}}\left(d_{1}, \ldots, d_{r}\right)$, onde $d_{1}+\cdots+d_{r}=n$ e a sequência $d=\left(d_{1}, \ldots, d_{r}\right)$ descreve as multiplicidades dos $r$ autovalores de $\xi$. O mapa 
momento ótimo é uma aplicação definida em

$$
J: \mathfrak{s u}^{*}(n) \longrightarrow \mathfrak{s u}^{*}(n) / A_{S U(n)}^{\prime}
$$

assim como no caso de $S U(2)$, para todo $\rho \in \mathfrak{s u}^{*}(n) / A_{S U(n)}^{\prime}$ temos que $S U(n)_{\rho}=S U(n)$ pois a ação é a coadjunta e a órbita coadjunta coincide com a folha simplética, assim, $S U(n) \cdot \rho=\mathcal{O}_{\rho}=\rho \in \mathfrak{s u}^{*}(n) / A_{S U(n)}^{\prime}$, dessa forma as condições da Observação 7.39 estão sendo satisfeitas e portanto o quociente

$$
M_{\rho}=M_{\mathcal{O}_{\rho}}:=J^{-1}\left(\mathcal{O}_{\rho}\right) / S U(n)=J^{-1}(\rho) / S U(n)
$$

tem uma estrutura simplética. O Teorema 7.40 mostra que o espaço reduzido $\left(J^{-1}(\rho), \omega_{\rho}\right)$ é folha simplética da estrata

$$
\mathbb{F}_{\mathbb{C}}\left(d_{1}^{j}, \ldots, d_{r_{j}}^{j}\right)=c\left(\mathfrak{s u}(n)_{\left(G_{d^{j}}\right)} / S U(n)\right), d^{j}=\left(d_{1}^{j}, \ldots, d_{r_{j}}^{j}\right)
$$

passando por $[m]$ em que $\rho=J(m)$ e $d^{j}=\left(d_{1}^{j}, \ldots, d_{r_{j}}^{j}\right)$ descreve as multiplicidades dos autovalores de $m$. 


\section{8 \\ Anexo}

Reservamos este capítulo para enunciar resultados que são utilizados nesta tese e demonstrar alguns deles. Dentre os resultados apresentados temos aquele que fornece condições para que o quociente $G / K$ seja um grupo de Lie, sendo $G$ um grupo de Lie e $K$ um subgrupo. Em seguida abordamos a respeito da forma de Killing e damos uma atenção especial aos grupos de Lie semisimples, fornecemos alguns exemplos de grupos de Lie que são semisimples com suas respectivas forma de Killing, e finalmente chegamos em um dos principais objetivos deste capítulo, que é concluir de que para grupos de Lie semisimples as representações adjunta e coadjunta podem ser identificadas.

Proposição 8.1 Seja K um subgrupo de Lie fechado de um grupo de Lie G. Então,

1. A ação a direita $G \times K \longrightarrow G$ é livre e própria.

2. $O$ espaço de órbitas $G / K$ tem uma única estrutura suave tal que a aplicação quociente $\pi: G \longrightarrow G / K$ é sobrejetiva. Além disso,

$$
\operatorname{dim}(G / K)=\operatorname{dim}(G)-\operatorname{dim}(K)
$$

Se $K$ é subgrupo normal de $G$, então o quociente é um grupo com multiplicação definida por

$$
\left[g_{1}\right]\left[g_{2}\right]=\left(g_{1} K\right)\left(g_{2} K\right)=g_{1} g_{2} K
$$

Neste caso podemos perguntar se $G / K$ é um grupo de Lie. Se $K$ é fechado, sabemos que $G / K$ é uma variedade suave e que a aplicação quociente é suave. De fato temos o seguinte resultado:

Proposição 8.2 Se $K$ é um subgrupo normal fechado de um grupo de Lie $G$, então $G / K$ é um grupo de Lie e a aplicação quociente $G \longrightarrow G / K$ é um homomorfismo de grupos de Lie.

Para mais detalhes destes resultados o prezado leitor pode consultar [23], Proposições 5.123 e 5.124 
Um outro resultado que diz a respeito do espaço de órbitas quando a ação do grupo é uma ação própria é o seguinte

Proposição 8.3 Se um grupo de Lie age continuamente e propriamente em uma variedade, então o espaço de órbitas é Hausdorff.

Este resultado está demonstrado em [14] na página 543.

\section{1}

\section{Álgebras de Lie e a forma de Killing}

Definição 8.4 Uma álgebra de Lie $\mathfrak{g}$ é chamada

- Simples, se não é abeliana e não contém ideal não trivial.

- Semisimples, se não contém ideal abeliano não nulo.

- Solúvel se para algum n, o subespaço $\mathfrak{g}^{(n)}$, definido recursivamente por $\mathfrak{g}^{(k+1)}=\left[\mathfrak{g}^{(k)}, \mathfrak{g}^{(k)}\right]$ e $\mathfrak{g}^{(0)}=\mathfrak{g}$, satisfaz $\mathfrak{g}^{(n)}=\{0\}$.

- Nilpotente se para algum n, o subespaço $\mathfrak{g}_{(n)}$, definido recursivamente por $\mathfrak{g}_{(k+1)}=\left[\mathfrak{g}, \mathfrak{g}_{(k)}\right]$ e $\mathfrak{g}_{(0)}=\mathfrak{g}$, satisfaz $\mathfrak{g}_{(n)}=0$.

Um grupo de Lie $G$ é chamado simples, semisimples, solúvel ou nilpotente se a sua álgebra de Lie é simples, semisimples, solúvel ou nilpotente respectivamente.

Definição 8.5 (Forma de Killing) A forma de Killing de uma álgebra de Lie de dimensão finita $\mathfrak{g}$ é a forma bilinear

$$
k: \mathfrak{g} \times \mathfrak{g} \rightarrow \mathbb{R}, k(X, Y):=\operatorname{tr}\left(\operatorname{ad}_{X} \operatorname{ad}_{Y}\right)
$$

Observação 8.6 A forma de Killing é bilinear, de fato, note primeiramente que para $X, Y, Z, W \in \mathfrak{g}$ temos que

$$
\begin{aligned}
\operatorname{ad}_{X+Y} \operatorname{ad}_{Z} W & =\operatorname{ad}_{X+Y}[Z, W] \\
& =[X+Y,[Z, W]] \\
& =[X,[Z, W]]+[Y,[Z, W]] \quad \text { bilinearidade do }[\cdot, \cdot] \\
& =\operatorname{ad}_{X} \operatorname{ad}_{Z} W+\operatorname{ad}_{Y} \operatorname{ad}_{Z} W
\end{aligned}
$$

dessa forma,

$$
\begin{aligned}
k(X+Y, Z) & =\operatorname{tr}\left(\operatorname{ad}_{X+Y} \operatorname{ad}_{Z}\right) \\
& =\operatorname{tr}\left(\operatorname{ad}_{X} \operatorname{ad}_{Z}+\operatorname{ad}_{Y} \operatorname{ad}_{Z}\right) \\
& =\operatorname{tr}\left(\operatorname{ad}_{X} \operatorname{ad}_{Z}\right)+\operatorname{tr}\left(\operatorname{ad}_{Y} \operatorname{ad}_{Z}\right), \text { pois } \operatorname{tr}(A+B)=\operatorname{tr} A+\operatorname{tr} B \\
& =k(X, Z)+k(Y, Z)
\end{aligned}
$$

analogamente faz-se para a segunda entrada, mostrando assim que $k$ é bilinear. 
O resultado a seguir apresenta duas propriedades a respeito da representação adjunta Ad e da aplicação linear ad, as quais usaremos mais a frente.

Proposição 8.7 Sejam G um grupo de Lie e $\mathfrak{g}$ a sua álgebra de Lie. Temos que para todo $X, Y \in \mathfrak{g}$ e $g \in G$ ocorre

$$
\begin{aligned}
& \text { 1. } \operatorname{Ad}_{g} \operatorname{add}_{X} \operatorname{Ad}_{g^{-1}}=\operatorname{add}_{A_{g} X} \\
& \text { 2. } \operatorname{ad}_{[X, Y]}=\operatorname{ad}_{X} \operatorname{ad}_{Y}-\operatorname{ad}_{Y} \operatorname{ad}_{X}
\end{aligned}
$$

Proof. Fixando $Y \in \mathfrak{g}$, temos

$$
\begin{aligned}
\operatorname{Ad}_{g} \operatorname{ad}_{X} \operatorname{Ad}_{g^{-1}} Y & =\operatorname{Ad}_{g}\left[X, \operatorname{Ad}_{g^{-1}} Y\right] & & \operatorname{pois~ad}_{X} Y=[X, Y] \\
& =\left[\operatorname{Ad}_{g} X, \operatorname{Ad}_{g} \operatorname{Ad}_{g^{-1}} Y\right] & & \text { Proposição 2.18 } \\
& =\left[\operatorname{Ad}_{g} X, Y\right] & & \text { Proposição 2.18 } \\
& =\operatorname{ad}_{\operatorname{Ad}_{g} X} Y & &
\end{aligned}
$$

provando assim 1. Agora fixando $Z \in \mathfrak{g}$, temos

$$
\begin{array}{rlrl}
\left(\operatorname{ad}_{X} \operatorname{ad}_{Y}-\operatorname{ad}_{Y} \operatorname{ad}_{X}\right)(Z) & =\operatorname{ad}_{X} \operatorname{ad}_{Y} Z-\operatorname{ad}_{Y} \operatorname{ad}_{X} Z & \\
& =\operatorname{ad}_{X}[Y, Z]-\operatorname{ad}_{Y}[X, Z] & & \text { pois } \operatorname{ad}_{X} Y=[X, Y] \\
& =[X,[Y, Z]]-[Y,[X, Z]] & \\
& =-[Z,[X, Y]] & & \text { id. Jacobi para }[\cdot, \cdot] \\
& =[[X, Y], Z] & & \text { antissimetria de }[\cdot, \cdot] \\
& =\operatorname{ad}_{[X, Y]} Z &
\end{array}
$$

provando assim 2 .

Proposição 8.8 Seja $\mathfrak{g}$ uma álgebra de Lie, seja $\mathfrak{a}$ um ideal em $\mathfrak{g}$ e sejam $k_{\mathfrak{g}}$ e $k_{\mathfrak{a}}$ as respectivas formas de Killing. Então $\forall X, Y \in \mathfrak{a}$ vale

$$
k_{\mathfrak{a}}(X, Y)=k_{\mathfrak{g}}(X, Y)
$$

Proof. Para mostrar este resultado usaremos o seguinte resultado:

Se $W \subset V$ é um subespaço, onde $V$ tem dimensão finita e $T: V \rightarrow V$ um endomorfismo tal que $T(V) \subset W$, então $\operatorname{tr}(T)=\operatorname{tr}\left(\left.T\right|_{W}\right)$.

Dessa forma, sejam $X, Y \in \mathfrak{a}, \log o$

$$
\operatorname{ad}_{X} \operatorname{ad}_{Y}: \mathfrak{g} \longrightarrow \mathfrak{g}
$$

é um endomorfismo tal que

$$
\left(\operatorname{ad}_{X} \operatorname{ad}_{Y}\right)(\mathfrak{g}) \subset \mathfrak{a}
$$


e pelo resultado mencionado temos que:

$$
k_{\mathfrak{g}}(X, Y)=\operatorname{tr}\left(\operatorname{ad}_{X} \operatorname{ad}_{Y}\right)=\operatorname{tr}\left(\left.\left(\operatorname{ad}_{X} \operatorname{ad}_{Y}\right)\right|_{\mathfrak{a}}\right)=\operatorname{tr}\left(\left.\left.\operatorname{ad}_{X}\right|_{\mathfrak{a}} \operatorname{ad}_{Y}\right|_{\mathfrak{a}}\right)=k_{\mathfrak{a}}(X, Y)
$$

Proposição 8.9 Seja $k$ a forma de Killing de $\mathfrak{g}$ com núcleo

$$
\operatorname{ker}(k):=\{X \in \mathfrak{g} \mid k(X, Y)=0, \forall Y \in \mathfrak{g}\}
$$

Então $\operatorname{ker}(k)$ é um ideal de $\mathfrak{g}$.

Proof. Seja $X \in \operatorname{ker}(k)$ e $Y \in \mathfrak{g}$, precisamos mostrar que $[X, Y] \in \operatorname{ker}(k)$, para isto, note que

$$
\begin{aligned}
k([X, Y], Z) & =-k([Y, X], Z) & & \text { para qualquer } Z \in \mathfrak{g} \\
& =-k\left(\operatorname{ad}_{Y} X, Z\right) & & \text { pois } \operatorname{ad}_{Y} X=[Y, X] \\
& =k\left(X, \operatorname{ad}_{Y} Z\right) & & \text { por }(8.2) \\
& =0 & & \text { pois } X \in \operatorname{ker}(k)
\end{aligned}
$$

portanto $[X, Y] \in \operatorname{ker}(k), \operatorname{logo} \operatorname{ker}(k)$ é um ideal de $\mathfrak{g}$.

Para demonstrar a última parte da Proposição 8.14, precisaremos de alguns resultados, os quais apenas enunciaremos, para melhores esclarecimentos a respeito deste, o prezado leitor pode consultar [19], página 247.

Proposição 8.10 Todo ideal solúvel não nulo contém um ideal Abeliano não nulo.

Proposição 8.11 Uma álgebra de Lie é semisimples se, e somente se, não tem ideais solúveis não nulos.

Observação $\mathbf{8 . 1 2}$ Ideais de $\mathfrak{g}$ são em particular álgebras de Lie.

Proposição 8.13 (Primeiro critério de Cartan) Uma álgebra de Lie g é solúvel se, e somente se, $k\left(\mathfrak{g}, \mathfrak{g}^{(1)}\right)=0$.

Tendo enunciado esses resultados, podemos agora provar algumas propriedades da forma de Killing.

Proposição 8.14 (Propriedades da forma de Killing) Seja $G$ um grupo de Lie e seja $\mathfrak{g}$ a álgebra de Lie de G. A forma de Killing $k$ de $\mathfrak{g}$ é simétrica, Ad - invariante,

$$
k\left(\operatorname{Ad}_{g} X, \operatorname{Ad}_{g} Y\right)=k(X, Y)
$$


e satisfaz

$$
k\left(\operatorname{ad}_{Z} X, Y\right)+k\left(X, \operatorname{ad}_{Z} Y\right)=0 .
$$

É não degenerada se, e somente se, $\mathfrak{g}$ (e consequentemente $G$ ) é semisimples. Esta última é conhecida como Segundo Critério de Cartan.

Proof. Claramente a forma de Killing é simétrica, pois

$$
k(X, Y)=\operatorname{tr}\left(\operatorname{ad}_{X} \operatorname{ad}_{Y}\right)=\operatorname{tr}\left(\operatorname{ad}_{Y} \operatorname{ad}_{X}\right)=k(Y, X) .
$$

Mostremos agora que $k$ é Ad -invariante

$$
\begin{aligned}
k(X, Y) & =\operatorname{tr}\left(\operatorname{ad}_{X} \operatorname{ad}_{Y}\right) & & \\
& =\operatorname{tr}\left(\operatorname{Ad}_{g} \operatorname{ad}_{X} \operatorname{ad}_{Y} \operatorname{Ad}_{g^{-1}}\right) & & \text { pois } \operatorname{tr}(A B)=\operatorname{tr}(B A) \\
& =\operatorname{tr}\left(\operatorname{Ad}_{g} \operatorname{ad}_{X} \operatorname{Ad}_{g^{-1}} \operatorname{Ad}_{g} \operatorname{ad}_{Y} \operatorname{Ad}_{g^{-1}}\right) & & \\
& =\operatorname{tr}\left(\operatorname{ad}_{\operatorname{Ad}_{g} X} \operatorname{add}_{A_{g} Y}\right) & & \text { item 1 do Lema 8.7 } \\
& =k\left(\operatorname{Ad}_{g} X, \operatorname{Ad}_{g} Y\right) & & \text { definição de } k
\end{aligned}
$$

portanto $k$ é Ad-invariante. Mostremos agora que

$$
\begin{aligned}
& k\left(\operatorname{ad}_{Z} X, Y\right)+k\left(X, \operatorname{ad}_{Z} Y\right)=0 \\
& k\left(\operatorname{ad}_{Z} X, Y\right)=k([Z, X], Y) \\
& \text { pois } \operatorname{ad}_{Z} X=[Z, X] \\
& =\operatorname{tr}\left(\operatorname{ad}_{[Z, X]} \operatorname{ad}_{Y}\right) \\
& \text { definição de } k \\
& =\operatorname{tr}\left(\left(\operatorname{ad}_{Z} \operatorname{ad}_{X}-\operatorname{ad}_{X} \operatorname{ad}_{Z}\right) \operatorname{ad}_{Y}\right) \\
& \text { item } 2 \text { do Lema } 8.7 \\
& =\operatorname{tr}\left(\operatorname{ad}_{Z} \operatorname{ad}_{X} \operatorname{ad}_{Y}-\operatorname{ad}_{X} \operatorname{ad}_{Z} \operatorname{ad}_{Y}\right) \\
& =\operatorname{tr}\left(\operatorname{ad}_{X} \operatorname{ad}_{Y} \operatorname{ad}_{Z}-\operatorname{ad}_{X} \operatorname{ad}_{Z} \operatorname{ad}_{Y}\right) \text { pois } \operatorname{tr}(A B)=\operatorname{tr}(B A) \\
& =\operatorname{tr}\left(-\operatorname{ad}_{X}\left(\operatorname{ad}_{Z} \operatorname{ad}_{Y}-\operatorname{ad}_{Y} \operatorname{ad}_{Z}\right)\right) \\
& =-\operatorname{tr}\left(\operatorname{ad}_{X} \operatorname{ad}_{[Z, Y]}\right) \\
& =-k(X,[Z, Y]) \\
& =-k\left(X, \operatorname{ad}_{Z} Y\right)
\end{aligned}
$$

portanto

$$
k\left(\operatorname{ad}_{Z} X, Y\right)+k\left(X, \operatorname{ad}_{Z} Y\right)=0
$$

Vamos agora provar a última parte, $k$ é não degenerada se, e somente se, $\mathfrak{g}$ é semisimples.

Defina

$$
\mathfrak{g}^{\perp}:=\{X \in \mathfrak{g} \mid k(X, \mathfrak{g})=0\},
$$

vamos mostrar que todo ideal abeliano $I$ de $\mathfrak{g}$ está contido em $\mathfrak{g}^{\perp}$. Sejam $X \in I$ e $Y \in \mathfrak{g}$, para mostrarmos que $X \in \mathfrak{g}^{\perp}\left(I \subset \mathfrak{g}^{\perp}\right)$, basta mostrar que

$$
k(X, Y)=\operatorname{tr}\left(\operatorname{ad}_{X}, \operatorname{ad}_{Y}\right)=0 .
$$


Para isto, seja $Z \in \mathfrak{g}$, assim

$$
\left(\operatorname{ad}_{X} \operatorname{ad}_{Y}\right)^{2}(Z)=[X,[Y,[X,[Y, Z]]]]
$$

note que devido $X \in I$, temos que $[X,[Y, Z]] \in I$ e $\log 0[Y,[X,[Y, Z]]] \in I$, como $I$ é abeliano, segue que

$$
[X,[Y,[X,[Y, Z]]]]=0
$$

dessa forma $\operatorname{ad}_{X} \operatorname{ad}_{Y}$ é nilpotente e portanto seu traço é zero. Assim $I \subset \mathfrak{g}^{\perp}$. Dessa forma se $k$ é não-degenerada temos que $\mathfrak{g}^{\perp}=\{0\}$, e portanto $\mathfrak{g}$ não contém ideal abeliano não nulo, logo $\mathfrak{g}$ é semisimples.

Reciprocamente, assuma que $\mathfrak{g}$ é semisimples, pela Proposição 8.9, temos que $\mathfrak{g}^{\perp}$ é um ideal de $\mathfrak{g}$. Isto implica que a forma de Killing (da álgebra de Lie) de $\mathfrak{g}^{\perp}$ é a restrição a $\mathfrak{g}^{\perp}$ da forma de Killing de $\mathfrak{g}$ e é consequentemente trivial. Agora, o primeiro critério de Cartan (proposição 8.13), (que diz que $k\left(\mathfrak{g}^{\perp},\left[\mathfrak{g}^{\perp}, \mathfrak{g}^{\perp}\right]\right)=0 \Leftrightarrow \mathfrak{g}^{\perp}$ é solúvel) afirma que $\mathfrak{g}^{\perp}$ é um ideal solúvel em $\mathfrak{g}$. Consequentemente, se $\mathfrak{g}^{\perp}$ fosse não nulo, pela proposição 8.10 conteria um ideal Abeliano não nulo, o que não ocorre pois $\mathfrak{g}$ é semisimples. Portanto, $\mathfrak{g}^{\perp}=\{0\}, \operatorname{logo} k$ é não degenerada.

Observação 8.15 As álgebras de Lie clássicas, tais como $\mathfrak{s l}(n), \mathfrak{s u}(n), \mathfrak{s p}(n)$, $\mathfrak{s o}(n)$ são semisimples.

Observação 8.16 Para cada grupo de Lie clássico semisimples, existe um fator $c>0$ tal que

$$
k(X, Y)=c \operatorname{tr}(X Y), \text { para todo } X, Y \in \mathfrak{g}
$$

- Para $\mathfrak{s l}(n)$ e $\mathfrak{s u}(n), n \geq 2$, o fator é $c=2 n$.

- Para $\mathfrak{s o}(n), n \geq 3$, o fator é $c=n-2$.

- Para $\mathfrak{s p}(n), n \geq 1$, o fator é $c=2(n+1)$.

Observação 8.17 No caso de grupos de Lie clássicos, como $S O(n)$ e $S U(n)$, temos que:

$$
\operatorname{Ad}_{g} X=g X g^{-1} e \operatorname{Ad}_{g}^{*} \xi=g^{-1} \xi g \text { onde } X \in \mathfrak{g} e \xi \in \mathfrak{g}^{*}
$$

temos assim que as órbitas adjunta e coadjunta coincidem.

Observação 8.18 Portanto, se $G$ é um grupo de Lie semisimples, ou seja, $\mathfrak{g}$ é uma álgebra de Lie semisimples, temos que a sua forma de killing $k$ é não degenerada, sendo assim, ela define um isomorfismo entre $\mathfrak{g}$ e $\mathfrak{g}^{*}$. 
Como consequência temos o seguinte resultado.

Observação 8.19 Se Gé um grupo de Lie semisimples, a forma de Killing é não degenerada e induz um isomorfismo linear $F: \mathfrak{g} \rightarrow \mathfrak{g}^{*}$ definido por $\langle F(X), Y\rangle=k(X, Y)$ para todo $X, Y \in \mathfrak{g}$. A Ad-invariância implica que

$$
F \circ \operatorname{Ad}_{g}=\operatorname{Ad}_{g}^{*} \circ F
$$

para todo $g \in G$. Assim $F$ é um isomorfismo das representações $\mathrm{Ad} e \mathrm{Ad}^{*}$ de G. Como uma consequência, para grupos de Lie semisimples, as representaçôes adjunta e coadjunta podem ser identificadas. 


\section{Referências bibliográficas}

[1] J.J. Duistermaat and J.A.C. Kolk, Lie groups, Universitext, Springer-Verlag, Berlin, 2000.

[2] J.E. Marsden and T.S. Ratiu, Reduction of Poisson manifolds, Lett. Math. Phys. 11 (1986), no. 2, 161-169.

[3] J.-P. Ortega, The symplectic reduced spaces of a Poisson action, C. $R$. Acad. Sci. Paris Sér. I Math. 334 (2002), 999-1004.

[4] J.-P. Ortega and T.S. Ratiu, The optimal momentum map, Geometry, mechanics, and dynamics, Springer, New York, 2002, pp. 329-362.

[5] J.-P. Ortega and T.S. Ratiu, Momentum Maps and Hamiltonian Reduction, Progress in Mathematics, volume 222. Birkhaüser Verlag, 2004.

[6] R. Sjamaar and E. Lerman, Stratified symplectic spaces and reduction, Ann. of Math. (2) 134 (1991), no. 2, 375-422.

[7] A. Cannas da Silva, Lectures on symplectic geometry, Lecture Notes in Mathematics, Published by Springer-Verlag as number 1764, 2006

[8] D. McDuff, D. Salamon Introduction to symplectic topology. Second edition. Oxford Mathematical Monographs. The Clarendon Press, Oxford University Press, New York, 1998.

[9] A. Mandini, The Duistermaat-Heckman formula and the cohomology of moduli spaces of polygons, Journal of Symplectic Geometry. 12.10.4310/JSG.2014.v12.n1.a6.

[10] R.L. Fernandes, J.-P. Ortega, and T.S. Ratiu. The momentum map in Poisson geometry. American journal of mathematics, 131 (5), 1261-1310.

[11] V. Guillemin and S. Sternberg, Convexity properties of the moment mapping, Invent. Math. 67 (1982), no. 3, 491-513.

[12] Dufour, J.P. e Zung, N.T. Poisson structures and their normal forms, Progress in Mathematics, vol. 242, Birkhäuser Basel, 2005. 
[13] Bursztyn, H. e Macarini, L. Introdução à Geometria Simplética, XIV Escola de Geometria Diferencial, IMPA, Rio de Janeiro, 2006.

[14] Lee, J.M. Introduction to Smooth Manifolds, vol. 218, Springer Verlag, 2003

[15] Marsden, J.E. e Ratiu, T.S. Introduction to mechanics and symmetry: a basic exposition of classical mechanical systems, vol. 17. Springer Verlag, 1999.

[16] Fernandes, R. L. e Marcut, I.. Lectures on poisson geometry, 2014. Accesed on march 2018

[17] Kirillov, A. Jr. An introduction to Lie groups and Lie algebras, Cambridge studies in advanced mathematics 113, Cambridge University Press, 2008

[18] Adler, M., Moerbeke, P. V e Vanhaecke, P. Algebraic Integrability, Painlevé Geometry and Lie Algebras, A Series of Modern Surveys in Mathematics, v. 47, Springer-Verlag Berlin Heidelberg, 2004

[19] Rudolph, G. e Schmidt, M. Differential Geometry and Mathematical Physics - Part I. Manifolds, Lie Groups and Hamiltonian Systems, Springer, 2013

[20] Holm, D. D., Schmah T. e Stoica C. Geometric mechanics and symmetry From finite to infinite dimensions Oxford Uiversity Press, 2009

[21] Cushman, R, Duistermaat. H e Sniatycki J. Geometry of Nonholonomically Constrained Systems Advenced series in nonlinear dynamics, v. 26, World Scientific, 2009

[22] Hall, B., Lie Groups, Lie Algebras, and Representations - An Elementary Introduction - Second Edition, Springer, 2015

[23] Lee, J. M., Manifolds and Differential Geometry Graduate Studies in Mathematics Volume 107, American Mathematical Society, 2009

[24] Tu, L. W., An introduction to manifolds, Second Edition, Springer, 2010.

[25] Baker, A., Matrix Groups - An Introdudion to Lie Group Theory, Springer Undergraduate Mathematics Series, Springer 2001.

[26] Pfeifer, W. The Lie Algebras $\mathfrak{s u}(n)$, Springer Basel AG. 
[27] Arathoon, P. Coadjoint orbits of the special Euclidean group. Masters thesis, Manchester Institute for Mathematical Sciences, The University of Manchester, 2015.

[28] R.L. Fernandes. A note on proper Poisson action. arXiv:math/0503147. 
A

\section{Notações}

Aqui listamos algumas notações usadas ao decorrer do texto.

- $\mathfrak{X}^{k}(M)$ campos multivetoriais de grau $k$

- $\Omega^{k}(M)$ formas diferenciais de grau $k$

- $\mathcal{L}_{X}$ derivada de Lie de formas ou campos multivetoriais ao longo de um campo vetorial $X$

- $i_{X} \omega$ produto interior de formas diferenciais por um campo vetorial $X$

- $X_{h}$ campo vetorial Hamiltoniano da função $h$

- $\{f, g\}$ colchete de Poisson das funções $f$ e $g$

- $[X, Y]$ colchete de Lie dos campos vetoriais $X$ e $Y$

- $\mathfrak{g}^{*}$ dual da álgebra de Lie $\mathfrak{g}$

- $\mathbb{F}_{\mathbb{C}}\left(d_{1}, \ldots, d_{r}\right)$ variedade bandeira complexa

- $\mathbf{A d}^{*}$ ação coadjunta diagonal

- $\mathcal{O}_{\xi}^{D}$ órbita coadjunta diagonal por $\xi$

- $\mathcal{C}^{\infty}(M)$ funções suaves em $M$ 